\title{
From sample to sensor
}

Citation for published version (APA):

Heidt, B. (2022). From sample to sensor: Usage and fabrication of point of care biosensor systems.

[Doctoral Thesis, Maastricht University]. Maastricht University. https://doi.org/10.26481/dis.20220126bh

\section{Document status and date:}

Published: 01/01/2022

DOI:

10.26481/dis.20220126bh

Document Version:

Publisher's PDF, also known as Version of record

\section{Please check the document version of this publication:}

- A submitted manuscript is the version of the article upon submission and before peer-review. There can be important differences between the submitted version and the official published version of record.

People interested in the research are advised to contact the author for the final version of the publication, or visit the DOI to the publisher's website.

- The final author version and the galley proof are versions of the publication after peer review.

- The final published version features the final layout of the paper including the volume, issue and page numbers.

Link to publication

\footnotetext{
General rights rights.

- You may freely distribute the URL identifying the publication in the public portal. please follow below link for the End User Agreement:

www.umlib.nl/taverne-license

Take down policy

If you believe that this document breaches copyright please contact us at:

repository@maastrichtuniversity.nl

providing details and we will investigate your claim.
}

Copyright and moral rights for the publications made accessible in the public portal are retained by the authors and/or other copyright owners and it is a condition of accessing publications that users recognise and abide by the legal requirements associated with these

- Users may download and print one copy of any publication from the public portal for the purpose of private study or research.

- You may not further distribute the material or use it for any profit-making activity or commercial gain

If the publication is distributed under the terms of Article $25 \mathrm{fa}$ of the Dutch Copyright Act, indicated by the "Taverne" license above, 


\section{Maastricht University}

\section{From Sample To Sensor}

Usage and Fabrication of Point of Care Biosensor Systems

\section{DISSERTATION}

to obtain the degree of Doctor at Maastricht University, on the authority of the Rector Magnificus, Prof.dr. Rianne M. Letschert in accordance with the decision of the Board of Deans, to be defended in public on Wednesday the 26th of January 2022, at 10:00 hours

$$
\text { by }
$$

Benjamin Heidt

Approved, after corrections,

Prof. dr. Rianne M. Letschert,

Rector Magnificus

2021

R3.0 / ISBN 978-3-00-071166-4 


\section{Supervisors:}

Prof. dr. Thomas Cleij

Dr. Bart van Grinsven

\section{Co-supervisor:}

Dr. Kasper Eersels

\section{Assessment Committee :}

Prof. dr. J. van den Berg (Chair)

Dr. C. Koopman

Dr. C. Padilla Diaz

Prof. dr. L. Lacerda Bueno, Universidade Federal de Minas Gerais, Brazil

Prof. dr. P. Lieberzeit, University of Vienna, Austria 
Dedicated to my mother, Tina Heidt. 


\section{Acknowledgements}

It seems like forever ago that I abandoned my career in economics to follow my true passion: Science. My journey into science and research was long and challenging but also unbelievable rewarding. And it could not have happened the way it did without a large amount of people supporting me along the way. Here I want to give my sincerest thanks to all of them.

First and foremost, I would like to thank my mother, for without her ongoing and unwavering support, changing my complete career and going on this journey would not have been possible in the first place.

I would like to express my deepest appreciation to Dr. Bart van Grinsven, who was an excellent supervisor during those years. During my studies, the group grew to an impressive size in a very short time and I can only imagine the pressure and workload he was under. Nevertheless, he did an amazing job of guiding me through my Ph.D. and was always there for advice. The same of course is to be said of Dr. Kasper Eersels, who was always there for feedback and insights on my work and took great care of me and the other Ph.D. students. Next to his support in all professional regards, I also want to thank him for the many amazing discussions about the nature of science and society, which I enjoyed a great deal. Both Bart and Kasper, the dynamic duo of Maastricht University, were always there with valuable suggestions and feedback, they gave me room to explore my own research ideas and develop myself as a scientist, while keeping me on track at the same time, for which I am deeply grateful. I would also like to thank Dr. Thomas Cleij for his guidance and essential feedback to my research which helped in connecting this thesis to a coherent whole. 
The German term for Ph.D. advisor is 'Doktorvater', literally 'Doctor Father'. If Bart and Kasper are my doctor-fathers, then without a doubt Debby Hewitt is my doctor-mom. Thanks to her I could focus on my research while she managed everything from ordering chemicals to making sure I take my leave every once in a while. She was always there to listen to every concern and worry and made sure everything was okay when she sensed I was a little bit too stressed, for which she will always have my greatest appreciation and thanks!

I would also like to extend my deepest gratitude to Dr. Erik Steen Redeker, for his support in difficult situations, feedback and insight, as well as giving me the opportunity of taking part in two amazing iGEM projects that were one of the highlights of this journey. I truly hope I will be able to supervise an iGEM team in the future so I can collaborate (and compete) with Erik and MSP-iGEM!

I am also extremely grateful to Dr. Ricardo Fujiwara and Dr. Vanessa Gomes Fraga of UFMG. They made my stay in Brazil a delight, supported me in every way possible and were the most amazing hosts, which showed me the life in Brazil, both inside and outside of the laboratory. These are memories I will always treasure.

I would also like to express my sincere thanks to Dr. Hanne Diliën for her encouragement, suggestions and feedback for my research and Dr. Kathia Jimenez Monroy who always had an open ear for my concerns and was eager to help in every situation, which is much appreciated!

A special thanks goes to Renato Rogosic, my business-partner at Flui.Go. He did an amazing job supporting me inside the company as well as the laboratory with countless good ideas, 
suggestions and hard work. Flui.Go, which is a big part of my journey, could not have been possible without him.

Many thanks go to my other Ph.D.-peers, Joseph Lowdon, Rocio Arreguin-Campos and Manlio Caldera for great research ideas, feedback and an all-around amazing time in- and outside of the laboratory.

I would also like to recognize the effort of the students of Flui.Go. The first generation of students: Renée-Claire Belting, Juliette Passariello-Jansen, David Dimech, Silvio Bonni, Saga Björnör. As well as the ones that joined later on: Hana Cvelbar, Tobias Hensel, Sean Lane, Robin Neven and Elias Garcia Verstraete for their outstanding work and creativity at Flui.Go as well as making it a joyful place I will always remember fondly.

A special thanks goes also to my dear friends Dr. Eduardo Bras and Silke Müller, for countless nights complaining about academia as well as the state of humanity. My 'muggle' friend Matthew Barton, as well as my brother Julian Heidt have my gratitude for taking my mind off of science every once in a while and keeping me grounded in the real world.

I would like to thank every one of my friends and family who endured me talking $90 \%$ of the time about science, while they were politely nodding and staring into the distance.

Lastly my gratitude goes to the roughly 2,4 trillion E.coli, bravely giving their live for mostly failed experiments. 
viii 


\section{Abstract}

Point-of-Care (PoC) diagnostics is a term concerning diagnostic methods and devices that can be conducted at the patient side in a fast and easy manner. This means they can be used in a wide set of contexts from global pandemics to environments in which healthcare infrastructure is not properly established. This doctoral thesis concerns itself with several aspects of Point-of-Care diagnostics.

In the first part, the actual use of $\mathrm{PoC}$ in low-income countries is analyzed and several bottlenecks are identified. For this, the whole value chain is examined, including the fundamental research, the market distribution and the end-user application of $\mathrm{PoC}$ diagnostics, in order to find the most important aspects contributing to its success or failure. Identified elements include problems with misguided research philosophies, problems with distribution and stock management as well as limited end-user trust. This part of the thesis unveils important aspects and design considerations that were essential for the consecutive chapters as it became evident that, for example, 3D printing can be a valuable tool for on-demand research and production of $\mathrm{PoC}$ devices in low-income countries.

The third chapter concerns itself with the heat-transfer method, a proof-of-application for its use in diagnosis of urinary tract infections is established, using samples of several volunteers of different genders. The method is shown to work in clinically relevant ranges down to $10^{4} \mathrm{CFU}$. Furthermore, it is shown that a fast sample analysis is necessary to guarantee correct diagnostics results. This shows that rapid $\mathrm{PoC}$ diagnosis on the spot is the best mode of action, especially in low-income countries where, as we showed in the first part, a proper cold chain is not necessarily guaranteed. 
New methods of microfluidic manufacturing, suitable for lowincome countries, are demonstrated in the consecutive chapters. First a Form2 3D printer is benchmarked for its capability to produce microfluidic channels, both inside and on the surface of the print. It was found that embedded channels are easier to produce the more orthogonally they are in respect to the buildplate, and the larger they are. Channels on the surface were tremendously easier to produce than channels embedded in the print. The size limit of embedded channels was $500 \mu \mathrm{m}$ compared to $250 \mu \mathrm{m}$ for surface channels. This finding was used to improve on 3D printed microfluidics further by the development of 'topographical vacuum sealing'. Here the channels are 3D printed as groves on the surface and then consecutively closed using a low-resolution vacuum forming process. In this process, a heated thermoplastic is stretched over the 3D printed substrate and a vacuum applied. With this, the thermoplastic is aligned to the macro-topography of the substrate but does not intrude into the micro-sized channel due to their small size. A wide range of surface geometries can be covered and several angles from $90^{\circ}$ to $270^{\circ}$ were tested. This technique enables the construction of open channels via 3D printing, which in turn allows smaller channel sizes while at the same time keeping the benefit of 3D printing, the ability to cover wide form factors, intact. 


\section{Contents}

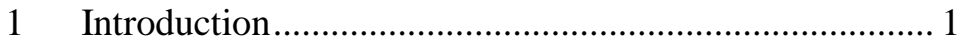

1.1 The Value of Diagnostic Devices for Society ......... 2

1.2 Biosensor Applications in Society …………............ 4

1.3 Elements of a (PoC-)Biosensor ................................. 6

1.3.1 The Recognition Layer........................................ 7

1.3.2 The Transducer ............................................... 16

1.3.3 Sample Management ........................................ 33

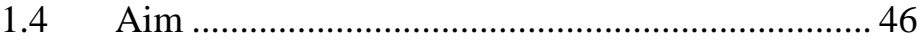

2 Point of Care Diagnostics in Resource-Limited Settings..

2.1 Chapter Context .................................................... 50

2.2 Introduction .......................................................... 50

$2.3 \quad$ Research ............................................................ 55

2.3.1 Fundamental Research: Funding Availability and Focus ..................................................................... 55

2.3.2 Proof-of-Concept and Prototypes: The Importance of Appropriate Device Characteristics....... 61

2.4 The Market.................................................................. 65

2.4.1 Market Introduction ........................................... 65

2.4.2 Market Penetration............................................. 69

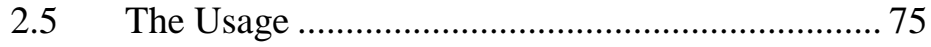

2.5.1 Healthcare Management .................................... 76

2.5.2 Patient Management............................................ 77

2.5.3 Training .......................................................... 80 


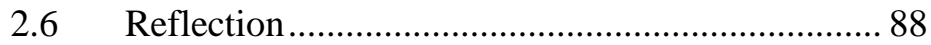

2.6.1 Main Findings …………………………........ 88

2.6.2 Outlook .......................................................... 90

$2.7 \quad$ Transition ........................................................... 93

3 Biomimetic Bacterial Identification Platform ................ 95

3.1 Chapter Context ..................................................... 96

$3.2 \quad$ Introduction.............................................................

3.3 Experimental Section ........................................... 100

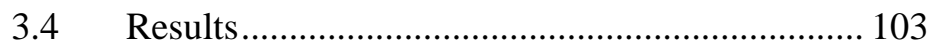

3.4.1 Surface Characterization and Calculation

Surface Coverage ......................................................... 103

3.4.2 Effect of Gender on Quantitative Detection of

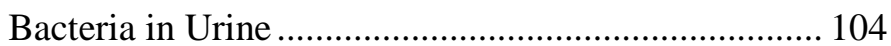

3.4.3 Reproducibility of Dynamic Sensor Range. 108

3.4.4 Potential Effects of Sample Storage and

Transport ...................................................................... 109

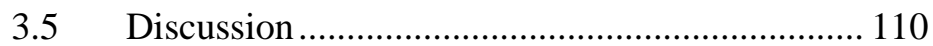

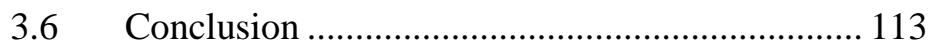

$3.7 \quad$ Transition ......................................................... 114

4 The Liberalization of Microfluidics ............................. 115

4.1 Chapter Context ................................................... 116

4.2 Introduction........................................................ 116

4.2.1 3D Printed Microfluidics ............................... 120

4.2.2 Accuracy in 3D Printing ............................... 121

4.2.3 Benchmark of the Form 2 SLA Printer....... 121 
4.3 Experimental Section ........................................... 122

4.3.1 Precision ....................................................... 122

4.3.2 Embedded Channel Accuracy ...................... 124

4.3.3 Open Feature Accuracy................................. 124

4.3.4 Optical Characteristics ................................. 124

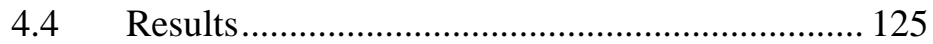

4.4.1 Open Channels .............................................. 125

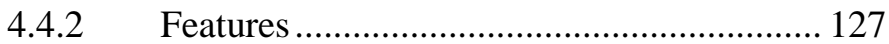

4.4.3 Closed Channels.............................................. 127

4.4.4 Optical Characteristics .................................. 128

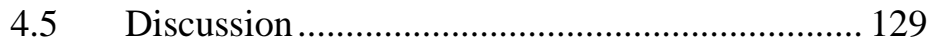

4.5.1 Performance of Different Resin Types ....... 129

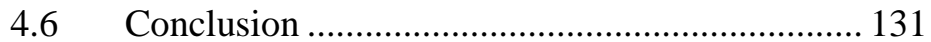

$4.7 \quad$ Transition .............................................................. 132

5 3D Printed Microfluidics .............................................. 133

5.1 Chapter Context .................................................... 134

5.2 3D Printed HTM-Flow Cells.................................. 135

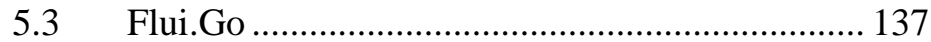

5.3.1 Flui.Go as a Company .................................. 142

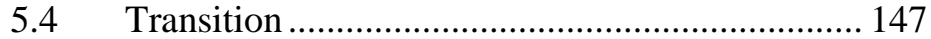

6 Topographical Vacuum Sealing ................................... 149

6.1 Chapter Context .................................................... 150

$6.2 \quad$ Introduction.......................................................... 151

6.3 Materials and Methods........................................... 153 
6.3.1 Printing and Vacuum Forming.................. 155

6.4 Benchmarking Devices .................................... 157

6.4.1 Angle Leakage ....................................... 157

6.4.2 Maximum Working Pressure ..................... 157

6.4.3 Column Separation................................... 158

6.4.4 Channel intrusion ..................................... 158

6.5 Results............................................................. 159

6.5.1 Angle Leakage ...................................... 159

6.5.2 Maximum Pressure ...................................... 161

6.5.3 Column Separation................................... 162

6.5.4 Channel Intrusion.................................... 164

6.6 Application Examples ...................................... 165

6.6.1 Application: Roundabout Serpentine Mixer 165

6.6.2 Application: UV-Vis Cuvette for Colorimetric Biosensing ........................................................... 166

6.6.3 Application: Luer-adapter......................... 169

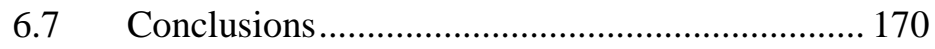

6.8 Transition ........................................................ 175

7 Conclusion and Final Thoughts ............................... 177

7.1 Aim of the Thesis............................................. 178

8 References....................................................... 183

8.1 References for Chapter 1: Introduction............... 183

8.2 References for Chapter 2: Point of Care Diagnostics in Resource-Limited Settings ........................................ 203 
8.3 References for Chapter 3: Biomimetic Bacterial Identification Platform ................................................... 216

8.4 References for Chapter 4: The Liberalization of

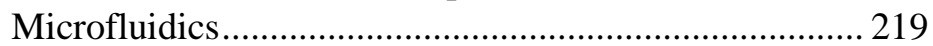

8.5 References for Chapter 5: 3D printed Microfluidics: Applications 222

8.6 References for Chapter 6: Topographical Vacuum Sealing 223

9 Supporting Information................................................. 229

9.1 Supporting Information : Chapter 3 …………..... 229

9.1.1 Study of the surface coverage of bacteria on the template stamp ......................................................... 229

9.2 Supporting Information: Chapter 4 …………...... 231

9.3 Supporting Information: Chapter 6 ……………. 234

10 Appendices........................................................ 240

10.1 Impact Paragraph ................................................. 240

10.2 Curriculum Vitae .................................................. 246 


\section{List of Figures}

Figure 1 - Google searches for the search terms 'PCR' and 'Rapid Test', data acquired via Google-Trends ........................ 3

Figure 2 - Schematic overview about the elements of a biosensor. .......................................................................... 7

Figure 3: Antibodies and antibody derived fragments............ 9

Figure 4: MIP Polymerization ................................................ 14

Figure 5- Different methods of SIP manufacturing .............. 16

Figure 6 - A lateral flow assay in normal (a.) and sandwich configuration (b.). The target analyte gets transported through the device by capillary forces in the nitro-cellulose membrane. 1. Target and detection-antibody conjugate at the conjugation pad. 2. Target binds to the recognition element. 3. Detectionantibody binds to the control-recognition-elements

Figure 7 - Example of an dipstick test: Glucose detection via glucose oxidase (GO) and horseradish-peroxidase (HRP) reactions with glucose and a chromogen respectively.......... 21

Figure 8 - Different approaches for fluorescent assays......... 23

Figure 9 - Different approaches for nucleic acid fluorescent probes............................................................................ 25

Figure 10 - The working mechanism of a toehold switch..... 26 
Figure 11 - SPR and 1SPR Methods...................................... 28

Figure 12 - Setup of a amperometric glucose sensor ............ 29

Figure 13 - Overview over the parts of a QCM (left) and a SAW (right) device

Figure 14- Schematics of the HTM with surface imprinted polymer as a receptor layer

Figure 15 - Photolithographic manufacturing of the master mold: 1. The silicon substrate. 2. SU-8 spin coated on top of the substrate. 3. The SU-8 gets exposed to UV-Light through a mask to cure it 4 . The uncured SU-8 is washed away.

Figure 16 - Soft lithography of the microfluidic channels: The finished mold is used (1.) and covered with liquid PDMS (2.), which is consecutively cured taking on the shape of the mold (3.). The PDMS is then plasma-bonded to a substrate to seal the channels (4.). 39

Figure 17 - Schematics of injection molding (left) and hot embossing (right) for microfluidic fabrication. 42

Figure 18 - Laminated microfluidic schematic. 43

Figure 19 - Schematic of a FDM Printer 45

Figure 20 - Resin printing methods: SLA (left) and LCD (right) 46

Figure 21 - Overview about the different chapters and their connection to the specific parts of the biosensor. 48 
Figure 22 - Migliozzi and Guibentif et al. looked at PoC bottlenecks from a technological standpoint. Figure re-used from [4] with permission under open-access creative commons copyright agreement

Figure 23 - Pai et al. investigated PoC use within different usage scenarios. Figure adapted from [5] with permission under open-access copyright agreement.

Figure 24 - Segmentation of stages a PoC device hast to pass to be able to bring a benefit to the patient. 55

Figure 25 - Identified Problems along the value chain. 86

Figure 26 - Identified Pivotal Points and Influencing Forces.

Figure 27 - Schematic of the thermal readout unit. The temperature of the heat provider (T1) is fully controlled by a thermocouple, a PID controller, and a heating element. A constant thermal current, is sent through the conducting chips and the SIP layer, resulting in a transmitted temperature profile registered by a second thermocouple. Reproduced with permission[23].Copyright 2017, American Chemical Society.

Figure 28 - Microscopic analysis of two E. coli SIPs at 50x magnification. a) Uncured and b) fully cross-linked SIP layer. 104

Figure 29 (a) and (c) - Time-dependent temperature response of an E. coli SIP in response to an increasing concentration of 
target bacteria. Picture showing the concentration dependent temperature decrease for both samples. 106

Figure 30 Dose-response curves of the time-dependent temperature data obtained in measurements using E. coli-SIPs. The relative response is plotted against the spiked concentration and a sigmoidal fit is used. Each curve (labeled sample I to IV) represents measurements on samples obtained from different volunteers. 109

Figure 31 - Comparison of the dose-response curves obtained with samples that were properly stored and measured (Sample I) and sample V, which represents measurements on samples that were stored for a day on different temperatures, simulating realistic conditions in current practice. ........................... 110

Figure 32 - A) VAT printing principle and B) FDM technology principle. 117

Figure 33 - A) Object printed with the Form 2 SLA printer (layer thickness $50 \mu \mathrm{m}$ ): the final part is much more detailed and the surface finish is smoother if compared with B) the same object printed with an FDM (Ultimaker 3, layer thickness $100 \mu \mathrm{m})$ printer. In the magnification, the stair-stepping lines are well visible. 119

Figure 34 - A) Printing object in different orientations has important effects on the end result. B) Test objects printed with different dimensions and orientations. C) Orientable stand for photoacquisition. D) Open channel features. 123

Figure 35 - Accuracy of open channel prints. A) Width accuracy of the three different resins for five channel width. B) 
Depth accuracy of the three resins in five different depths. C) Rendering of the printed part with indicated measuring area. D) Size accuracy of five different square sizes with the three different resin types. E) Depth accuracy of the squares for three different height types. F) Rendering of the test structure with marked areas of measurement

Figure 36 - Accuracy of 3D test prints with internal channels in four different channel sizes and five different angles. A) Clear resin, B) High Temperature resin, C) Tough resin, and D) rendering of the test model.

Figure 37 - A) Transmission values for the three different resins before and after the surface modification process. B) Comparison of transparency for the three different resin types before (left) and after (right) the surface modification, from left to right: : Clear, High Temperature, and Tough resin......... 129

Figure 38 - Parts of the new flow cell concept. a. lid containing the flow cell, b. aluminum substrate, c. heater-holder, d. heater, e. LEGO baseplate, $f$ and g. male and female LUER-to-tubing adapters, h. male LUER adapter with integrated bubble-trap, i. inclined ramp, j. holder for falcon tubes.

Figure 39 - Printed and assembled setup, arrested on a commercially available LEGO buildplate

Figure 40 - Schematic of the second generation Flui.Go block 139

Figure 41 - Overview of the different microfluidic building blocks 1. T-Connecter, 2. X-Connector, 3.Curve, 4 straight 
channel, 5. Reaction-block, 6. meander-mixer 7. Bubble expulsion.

Figure 42 - Example of added microfluidic networks for experiments Left: 3-component reaction chamber. Right: Droplet generator with collection container 140

Figure 43- Print setup in Preform, Red arrows represent escape path for uncured resin through the channels (print is upsidedown in bat configuration, red arrows pointing downwards).

Figure 44- Left and Middle: Assembled Flui.Go $\mathrm{pH}$ Experiment with the Flui.go blocks V.3. Right: an example of printed second generation of Flui.Go blocks.

Figure 45 - The advanced product, Pumping system with 2 pumps, syringes and waste container as well as the Flui.Gobricks, assembled into a version of the $\mathrm{pH}$-experiment ...... 144

Figure 46 -Mockup of a Flui.Go collection pack, for science education companies 146

Figure 47 - The fabrication process of topographical vacuum sealing. A thermoplastic is heated above its glass transition temperature and stretched over the topographic substrate. The vacuum aligns the thermoplastic to the surface of the substrate, while the microchannels remain free due to their small size. 154

Figure 48 - Testing device covering several challenging geometries and displaying the flexibility of the method..... 155 
Figure 49 - (I.) Rendering of a basic example of a testing chip with two crossings of a $270^{\circ}$ angle. The control channels are flanking the fluid channel and turn red if they fill due to leakage. (II.) Resulting 3D-print carrying water colored with red food coloring. (III.,IV.) Close-up of the angle crossing with control channels (C) and the fluid carrying channel (F).... 160

Figure 50 - Different structures for testing channel integrity while traversing different angles. Left: Rendering of the structure. Middle: Completed print sealed with the topographical vacuum forming method. Right: Magnified channels. (A) $330^{\circ}$ angle. (B) From left to right: $270^{\circ} 240^{\circ}$ and $210^{\circ}$ angles. (C) From left to right: $90^{\circ}, 120^{\circ}$ and $150^{\circ}$ angles. (D) From left to right: $90^{\circ}$ angles with $2 \mathrm{~mm}, 1.5 \mathrm{~mm}$ and $1 \mathrm{~mm}$

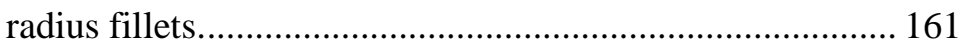

Figure 51 - Different structures for the maximum pressure test. Left: Rendering of the structures. Middle: Completed print, sealed with the topographical vacuum sealing method. Right: Pressurized channel with water. Top line: Monoplanar structure. Middle line: Prism-shaped structure with $135^{\circ}$ and $270^{\circ}$ angles. Bottom line: Cube extrusion with $90^{\circ}$ angle, $1 \mathrm{~mm}$ fillet and $270^{\circ}$ angle. 162

Figure 52 - Relationship between column height, column distance to each other and the height of the thermoplastic spanned in-between the columns. Heights at $0.5 \mathrm{~mm}$ made contact with the substrate, as this is the thickness of the thermoplastic sheets. The complete dataset as a $2 \mathrm{D}$ graph is available in the supplementary information......................... 163

Figure 53 - Thermoplastic intrusion into the channel as percentage of channel width. 164 
Figure 54 - A meander structure as a serpentine mixer going the whole way around a cube, increasing the usable space. 166

Figure 55 - A microfluidic 'cuvette' with the same width and height as a cuvette; this device can be used in any standard $\mathrm{UV} / \mathrm{Vis}$ spectrophotometer to conduct online measurements. It is filled with molecular imprinted polymers loaded with a malachite green, which is released on contact with the drug 2MXP. 168

Figure 56 - Absorbance measurement at $617 \mathrm{~nm}$ for the cuvetteshaped microfluidic structure, flushed consecutively with 0.3 , 0.6 and $1 \mathrm{mg} / \mathrm{mL} 2-\mathrm{MXP}$ solution. 168

Figure 57 - Channel with 3D-printed Luer-Adapter. The channel traverses completely on the upper surface and thus can be easily injection molded. 170 


\section{List of Abbreviations}

ABS

AM

ART

CFU

DLP

DNA

dsDNA

ELISA

FAD

FDM

FRET

GFP

HAI

HCT

HIC

HIV

HPV

HTM

ICT

LIC

IP

LCD

LCT

LHW

LOAC

LOAF
Acrylonitrile butadiene styrene

Additive manufacturing

Anti-retroviral therapy

Colony-forming unit

Digital light processing

Deoxyribonucleic acid

Double-stranded DNA

Enzyme-linked immunosorbent assay

Flavin adenine dinucleotide

Fused deposition modelling

Förster resonance energy transfer

Green fluorescent protein

Healthcare-acquired infections

High-complexity PoC tests

High-income country

Human immunodeficiency virus

Human papilloma virus

Heat-transfer method

Information and communication

technology

Low-income countries

Intellectual property

Liquid crystal display

Low complexity PoC tests

Lay healthcare worker

Lab-on-a-chip

Lab-on-a-foil 
LoD

LSPR

LTFU

$\mathrm{MiC}$

MIP

mSLA

NGO

PBS

PCR

PDMS

PETG

PLA

PMMA

PoC

PP

PQQ

QCM

qPCR

RHC

RNA

SAW

scvF

SDC

Sip

SLA

SPR

SSDNA

THF
Limit of detection

Localized surface plasmon resonance

Loss to follow up

Middle-income country

Molecularly imprinted polymer

Masked stereolithography

Non-government organization

Phosphate buffered saline

Polymerase chain reaction

Polydimethylsiloxane

Polyethylene terephthalate glycol

Polylactic acid

Polymethylmethacrylat

Point-of-Care

Poly-propylene

Pyrroloquinoline quinone

Quartz crystal microbalance

Quantitative polymerase chain reaction

Rural healthcare centers

Ribonucleic acid

Surface acoustic wave

Single chain variable fragment

Substrate displacement

colorimetry

Surface imprinted polymer

Stereolithography

Surface plasmon resonance

single stranded DNA

Tatrahydrofuran 
TOVAS

UTI

WHO
Topographical vacuum sealing

Urinary tract infection

World Health Organisation 


\section{Introduction}

The State of the Art of Biosensing - From Recognition Layer to Sample Management 


\subsection{The Value of Diagnostic Devices for Society}

Never has global travel been as easy and cheaply available as today. However, global travel also leads to global transmittance of infectious diseases and thus, never have infectious diseases been able to travel as far and as fast as today. This can result in immense social and economic threats, as was shown during the Covid-19 pandemic in the years 2020 and 2021. As of May 2021, the pandemic took more than 3.5 million lives $[1,2]$ and changed the world not just in health- but also in social and economic aspects [3]. Large social adjustments had to be made to prevent the spread of the virus, the uncertainty forced people in lockdowns, home-office and 'Zoom'-meetings, as no one could be sure who might be infected while being still asymptomatic. Hence, the invisibility of the virus became the biggest obstacle for a normal live. During this time, it became quite evident how immensely valuable diagnostics methods are to protect society from infectious disease. Before the year 2020, medical diagnostic devices and methods did not get much media attention. During the pandemic however, they became one of the spotlights. For the first time diagnostic procedures were widespread featured in media outlets and discussed broadly within society. This is illustrated in Figure 1, which shows the relative number of 'Google'-searches for the queries 'PCR' and 'Rapid Test'. 


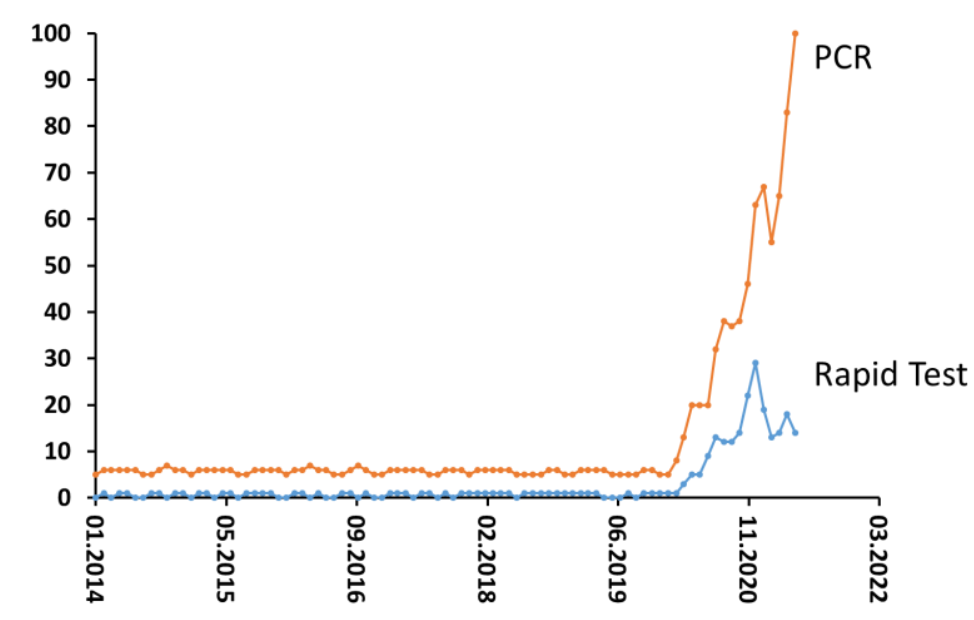

Figure 1 - Google searches for the search terms 'PCR' and 'Rapid Test', data acquired via Google-Trends

One of the foci was of course the quantitative polymerase chain reaction (qPCR) test, the standard test used for detection of viral nucleic acids. While the development of the testing method was fast [4], the increase in testing facilities lagged behind as countries tried to drastically increase the capabilities of their molecular biology laboratories [5]. Even after an increase in testing capabilities, qPCR-testing still presented a huge challenge in terms of logistics and workforce. qPCR-tests are (mostly) bound to laboratories and take time. The Infrastructure for sample collection in testing-centers had to be established and manned, transport to- and analysis in- the laboratory by trained personnel had to be conducted and digital infrastructure to inform the patients of testing result had to be maintained. A megaproject for any country. Laboratory-based tests however where not the only option and the benefits of 
rapid Point-of-Care (PoC) tests came into focus of the general public and their governments. PoC tests can be conducted directly patient-side and there is no necessity to send samples to an external laboratory, the result is usually available within minutes and the tests can be conducted with minimal training. While PoC tests are generally not as sensitive as laboratorybased tests, their ease of use, widespread availability and speed create a valuable first line of defense against pandemics, where the swiftness in which the result is attained is essential to prevent or mitigate the spread of the disease. Therefore, during the pandemic, PoC tests became widely used as a fast acting supplement for qPCR tests, as governments realized their huge potential $[6,7]$. The pandemic was a difficult challenge for society but it showed the extraordinary possibilities PoC tests offer, due to their speed, ease of use and applicability. However, global pandemics are not the only circumstance in which PoC devices can bring extraordinary benefit to society. Since they can be deployed with only minimal infrastructure requirements, are cheap, have a fast turnaround and are easy to use, they are ideal for countries that do not have a thorough healthcare infrastructure in place, like low-income countries (LIC). Therefore, research in- and effective use of PoC devices can be instrumental to improving healthcare and wellbeing around the world. However many groundbreaking PoC biosensor applications demonstrated in the laboratory have not yet found their way into society with two noteworthy exceptions: Lateral flow tests and blood glucose meters.

\subsection{Biosensor Applications in Society}

Lateral flow devices are the most common 'low-tech' PoC diagnostic device, and do not possess (in their basic form) any electronic parts. Their success is based on their affordability, ease of manufacturing, ease of use and independence of 
infrastructure such as electricity and a cooling chain, clear downsides however are their inability for reliable quantitative analysis and limited sensitivity [8]. Lateral flow devices are in essence a PoC version of an enzyme linked immunosorbent assay (ELISA). Similarly to an ELISA, immobilized antigens (for antibody assays) or antibodies (for antigen assays) are used, however in contrast to ELISA they are not immobilized in a microtiter plate, but on a nitrocellulose membrane. The sample is added to one end of the device and the capillary wetting of the membrane used to transport the sample to the recognition antibodies and antigens, where a red line will form if the target analyte is present, a complete explanation of the system can be found in the subsection Optical Transducers on page 17. While they are used successfully for the detection of infectious diseases such as HIV [9], Malaria [10] or more recently COVID-19 [11], the biggest example is their use in pregnancy tests [12], which have an estimated market volume of 1.29 billion US-dollar [13].

On the other hand, glucose meters are an example of a more 'high-tech' approach, being capable of analysis with higher sensitivity and accurate quantification, but requiring electricity and a readout screen for use. To use glucose meters, blood is taken via a finger prick an applied to a testing strip, which uses capillary forces to transport the blood to the sensor. The strip, which contains the electrodes and enzymes required for the electrochemical measurement, is placed into the readout device that uses an enzymatic recognition together with an amperometric transduction to quantitatively analyses the blood glucose content [14]. A more in-depth explanation of the working mechanism can be found in the subsection Electrochemical Transducers on page 28. The development of PoC glucose-measurement is an excellent example for the 
development of PoC devices over time; when first introduced PoC glucose meters had a weight of $1.2 \mathrm{~kg}$ [15], today small meters merely weigh 9 grams [15], and further developments created continuous blood glucose measurements which, while still invasive using a microneedle, measure the glucose levels continuously over the day and are connected to a smartphone [14]. Noninvasive techniques are also developed but are yet awaiting commercial application $[14,15]$. The great success of PoC use in blood glucose measurements helped alleviate the suffering of millions of people, as diabetes is one of the biggest health problems in developed nations, with up to 450 million affected in 2019 and an estimate of 700 million in 2045 [14, 16]. Diabetes care costs 245 billion US-dollar in the United States (2012), 43.2 billion Euro in Germany and 20.2 billion Euro in the UK (2010) the greatest share being inpatient-care costs [15]. Blood glucose meters on the other hand reached worldwide sales of 8.8 billion dollar in 2008, an compound annual growth rate of $12.5 \%$ since 1994 [17]. With this glucose meters account for $22 \%$ of the entire in-vitro diagnostic business [17].

\subsection{Elements of a (PoC-)Biosensor}

Those examples make it clear that PoC devices have large potential for personalized medicine, with biosensors forming the heart of many modern diagnostic PoC tools. Biosensors have been defined as devices that use biological or biomimetic recognition elements and can quantitatively or qualitatively recognize target analytes $[18,19]$. They can be separated into several different compartments (Figure 2): The recognition layer, the transducer and the readout system. The recognition layer binds the target analytes, the transducer translates the information of a binding event (the recognition) into a quantifiable signal that will be recorded by the readout element. 
Finally, an important addition to the biosensor system, especially for $\mathrm{PoC}$ use, is the sample management system, which provides an essential role for sample preparation, such as sample purification, matrix separation and reagent mixing as well as sample and reagent storage and transport to the sensor itself [20].

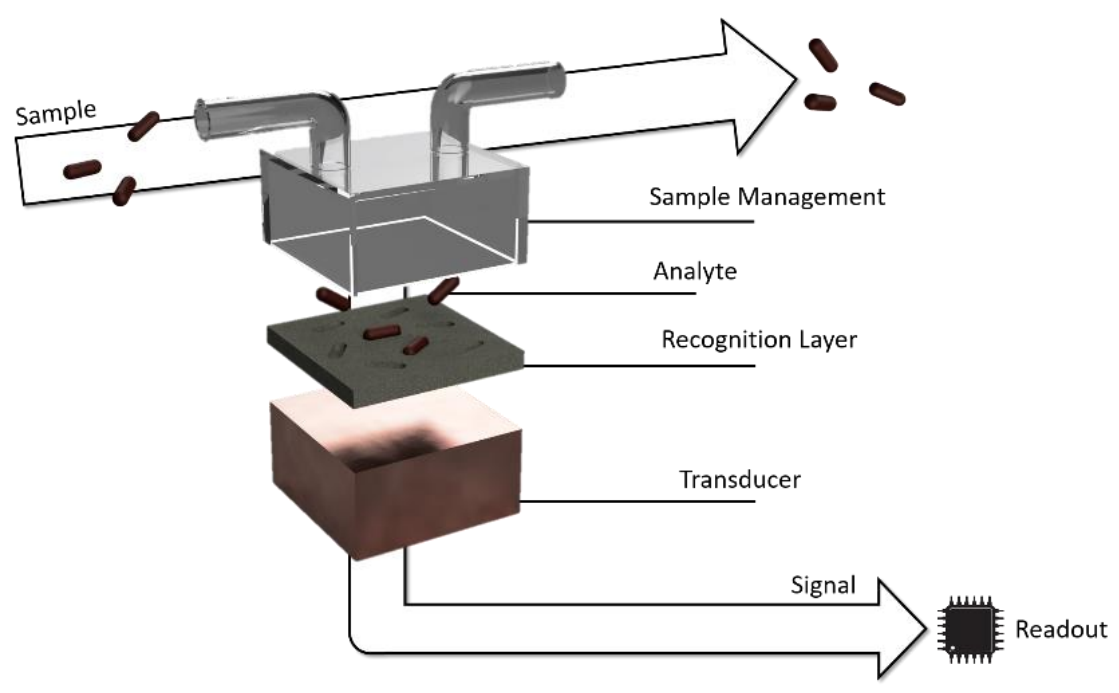

Figure 2 - Schematic overview about the elements of a biosensor.

\subsubsection{The Recognition Layer}

The recognition layer consists of biological or biomimetic recognition elements, which are able to bind the target analyte. There is a wide variety of recognition elements available and the choice is mainly depended on the target analyte, and the requirements of sensitivity and specificity as well as the matrix 
[21]. Biological recognition elements include proteins such as enzymes, antibodies and nanobodies; nucleic acids such as ssDNA/RNA and aptamers; or biomimetic recognition elements such as molecularly imprinted polymers and surface imprinted polymers. The use of the recognition layers is to specifically bind the analyte of interest and thus to recognize it and start the cascade of detection.

\subsubsection{Protein Based Recognition Elements: Antibodies, Enzymes and More}

\section{Antibodies}

One of the most used recognition elements in biosensors are antibodies. Since the natural purpose of antibodies is recognizing and binding antigens, their use as a recognition element in biosensor platforms was an obvious choice. Originally used for laboratory methods like Enzyme Linked Immunosorbent Assay (ELISA), antibodies were generated by the hybridoma method [22]. Here mice are injected with an antigen for which antibodies are to be produced. After an immune response, B-Lymphocytes are extracted out of the spleen and selected for the ones displaying antigens for the target. The B-lymphocytes are able to produce antibodies, however they are mortal and thus not usable for long term antibody production in bioreactors. To make them suitable for long-term production, they are fused with immortalized myeloma cells. The fused cells, now called hybridoma-cells, are selected via a HAT-selection medium and have the ability to produce antibodies (due to their B-cell parts) and are immortal (due to their myeloma parts) [22-25]. The cells can now be used in large-scale bioreactors to produce huge quantities of antibodies [23]. The invention of this method 
offered immense new possibilities for life science and thus the inventors, Niels Jerne, César Milstein and Georges Köhler where awarded the Nobel Prize for medicine in 1984 [24].

While often the whole antibody is used as a recognition element, it can be broken down to its different parts via enzymatic cleavage Figure 3, to yield smaller fragments that can be more efficient in therapeutic and diagnostic use due to their smaller size and lower immunogenicity [26, 27].

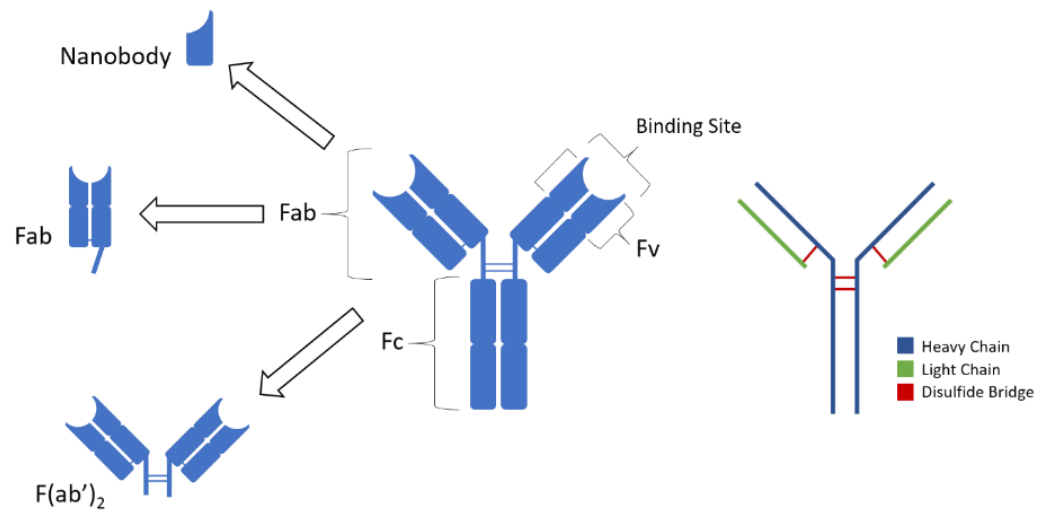

Figure 3: Antibodies and antibody derived fragments.

Thanks to the advances in genetics, molecular biology and biotechnology, new methods are being developed to forego the complex and labor intensive steps of the hybridoma technique. One common technique is phage-display [28-30]. This method uses phages, typically M13 phages which only infect F-Pili expressing E.coli [31], to display the binding-site of an antibody (mostly in the form of an single chain variable fragment $(\mathrm{scvF}$, a fusion protein of single and heavy chain variable fragment) on the outside of its protein hull. A library of antigen-binding-sites is used to create phages with a wide 
variety of different binding proteins. The phages are grown in bacteria and then extracted and exposed to the target-antigen, which is immobilized on a surface. The surface is washed consecutively, removing all phages with binding-sites that are unable to bind the target analyte. The phages capable to attach to the target are eluted in a later step and regrown in bacteria. This process is repeated until the binding-site with the highest affinity for the target is the only one remaining [29, 31]. The DNA-sequence of the found binding-site can then be implemented into an antibody sequence and the antibody expressed in bioreactors, alternatively the $s c v F$ can be produced in a bioreactor and used on their own. The benefit of this technique is that it directly connects the pheno- to the genotype and thus selection can easily be done via phenotype and the connected genotype exploited for further use [31].

\section{Enzymes}

This category uses the catalytic nature of enzymes to detect analytes, this is usually accomplished by using the target analyte as substrate for the enzyme while measuring a product of the catalytic reaction [21]. The biggest example in this group is also the most successful commercial example of biosensors and their point of care detection in general: glucose detection. Here either glucose oxidase or glucose dehydrogenase is used to start a signaling cascade that is typically monitored using an amperometric readout approach [14]. The benefit of enzymatic sensing lays in the wide availability of existing enzymes for biological relevant molecules. Similar to the glucose sensor based on glucose oxidase, several other oxidases can be used in the same way for detection of their specific substrate, such as lactate via lactate oxidase, alcohols via alcohol oxidases and so on [32]. Examples for the reactions occurring with these kinds 
of recognition elements will be shown more in depth in the chapter Electrochemical Transducers on page 28.

\subsubsection{Nucleic Acid Based Recognition elements ssDNA/RNA}

Nucleic acid based recognition elements are mostly used for the detection of other nucleic acids due to the inherent affinity of single stranded DNA / RNA to their matching strand [21]. Single stranded DNA (ssDNA) for example will hybridize with other ssDNA/RNA found in a sample, which has a complementary nucleotide sequence. The readout of this hybridization can be done label-free for example via impedance analysis [33, 34] or heat-transfer method [35] or more common by using fluorescent probes, as is common in qPCR, which will be elaborated on in the section Optical Transducers under Fluorescence on page 21. However, the inherent 'programmability' of DNA can also be exploited by other methods than simply using a complementary sequence as recognition element. One example is the toehold switch which uses binding to automatically activate protein expression and will be covered under the section Optical Transducers as well [36].

\section{Aptamers}

Nucleic acids as recognition elements can also be used for the recognition of whole cells, proteins, peptides and small molecules in the form of aptamers. Aptamers are about 100 base pairs long oligonucleotides with a 20-70 base pair long binding region [21,37] that can bind to proteins, and can be designed using a method coined Systemic Evolution of Ligands by Exponential Enrichment (SELEX) [21, 37-39]. Here a 
library of sequences is incubated with an immobilized target analyte. Some of the oligo-nucleotides are able to bind the target analyte and remain in the vessel upon washing, which removes all non-binding sequences. Now the sequences which were able to bind can be eluted and amplified via polymerase chain reaction (PCR), mutations can be induced and the oligonucleotides are allowed to bind the immobilized targets again. After washing, the sequences with decreased binding will be removed, while the ones with increased binding remain. This process can be repeated similarly to the bio-panning in phage display, to artificially evolve sequences with high binding affinity to the target analyte [37, 38]. Given that aptamers, similarly to Fab fragments are much smaller in comparison with antibodies (about 1-2 nm compared to about $10-15 \mathrm{~nm}$ of antibodies) a higher density of binding sites on the surface can be achieved [21, 40].

\subsubsection{Biomimetic Receptor layers}

Biomimetics describes the use of technology to emulate nature [41]. In the form of receptor layers this means synthetic materials get used to replace the traditionally used biological receptors such as antibodies and enzymes. Compared to them, biomimetic materials have a host of benefits. Biological elements evolved in nature to work in a very narrow range of physiological parameters. Most importantly the $\mathrm{pH}$-range of the solution, which often has to closely resemble physiological ranges to prevent denaturation of the biological receptor elements [42]. Furthermore, biological receptors prohibit the use of organic solvents and are prone to degradation over time. This and the complex production procedure are downsides that can be overcome with the use of biomimetic receptors [42, 43]. 
Here molecularly imprinted polymers (MIPs) and surface imprinted polymers (SIPs) can be used as an alternative to biological elements. Nicknamed 'plastic antibodies' [44], both are based on synthetic polymers and offer, compared to biological elements, extraordinary stability and resistance to wear and can be used in a wide range of solvents and $\mathrm{pH}$-ranges [43].

\section{MIPS}

For MIP fabrication, monomers are cross-linked in presence of the target analyte, forming the polymer around it, as seen in Figure 4 . While the polymer is formed, the functional groups of the monomers arrange themselves and form non-covalent bonds such as electrostatic interactions like hydrogen bonds and Van der Waals forces with the template [45]. When the template is later removed, the now cross-linked polymer still holds this shape, and can thus similarly to an antibody rebind the target molecule [21, 46]. The fine-tuning of monomer-type and cross-linker, reagent ratios and polymerization methods allows MIPs to be tailored to its specific application, which also gives them a superior reproducibility compared to other recognition elements [21, 47]. The polymerization can be conducted in various different ways, from Sol-Gel processes over polymerization in emulsions or suspensions to bulk polymerization. 
Introduction

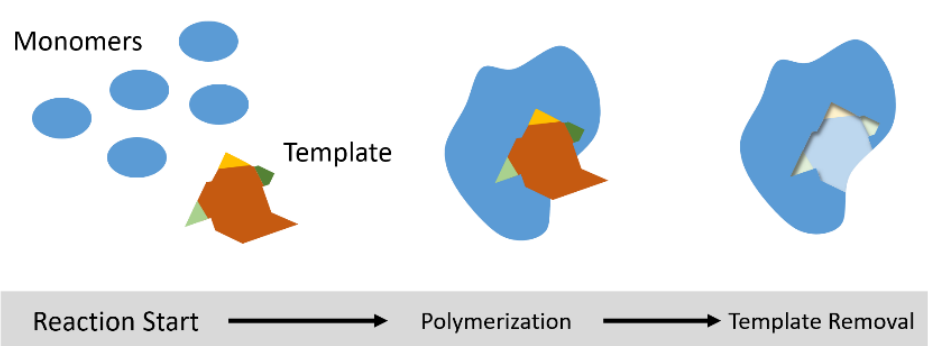

Figure 4: MIP Polymerization

SIPS

Similarly to MIPs, SIPs can also be produced using polymerization of a monomer but where MIPs are employed for small molecules, SIPs are better suited for large molecules like proteins and even whole biological cells. SIPs show a clear benefit with those analytes as it is much easier to remove the template, which are in the case of SIPs, as the name suggests, located on the surface of the polymer, thus removing the template and rebinding the analyte becomes easier [43]. SIPs can be manufactured in different ways, most commonly used are self-assembly techniques, molding techniques and microcontact imprinting, as seen in Figure 5. Self-assembled SIPs are fabricated similarly to the classical MIPs, as the template is placed in solution together with monomers and cross-linkers. During the polymerization, the monomers self-assemble around the template. Templates at the surface can then be removed, leaving cavities open for rebinding, however some of the template may end up in the bulk material where removal is not possible. Self-assembly methods have been used for templates such as enzymes [43, 48, 49] and self-assembled monolayers have been used for example for other proteins and virions $[42,43,50]$. Self-assembly can be supported by adding 
external forces upon monomers and templates, here one example is the use of electropolymerisation, polypyrolle films have been assembled via electropolymerisation and used, among others, the bacterium $P$. aeruginosa as a template, the negative charge of the template helped to attract it towards the growing polymeric structure and it could consecutively be removed by reversing the charge $[43,51,52]$.

Another large group of SIP manufacturing techniques, are molding methods. Here the template gets immobilized on a substrate and consecutively a low molecular weight polymer mixture is applied and cross-linked. Two methods are employed: For one the polymer can be cured on top of the immobilized substrate, creating the cavities on the underside, after crosslinking the polymer layer then is removed from the substrate-template layer and then applied to its place of use (Figure 5, 'molding'). This has been demonstrated with bacteria and mammalian cells as template [43, 53]. However, with this approach the polymer has to be removed from the template-substrate. If the polymer has to be reapplied, for example on top of a transducer surface, this can lead to problems. The second approach is to immobilize the template on the transducer surface itself apply the polymer in a way that it only partially submerges the template (Figure 5, 'direct molding'). This was done, for example, with hemoglobin [43, $54]$ and virions as a template $[43,55]$.

The final major SIP manufacturing method is stamp- or micro contact-imprinting. Here a pre-cured polymer is applied in a thin layer to one substrate (for example via spin-coating on the transducer surface in biosensors), while the template is applied to another substrate (the stamp). The stamp then gets brought into contact with the polymer surface, resulting in the templates 
slightly intruding in the not yet fully cross-linked polymer. After the stamp is applied, the polymer gets fully cross-linked around the template, which can then be removed while the polymer retains the binding cavities. Originally used to imprint yeast-cells $[43,56]$, the technology was further developed to imprint a wide array of targets, among them proteins [57-59], viruses [57, 60], bacteria [61-63], and mammalian cells [6466]. Micro contact-imprinting is now one of the most used SIP manufacturing methods [67].

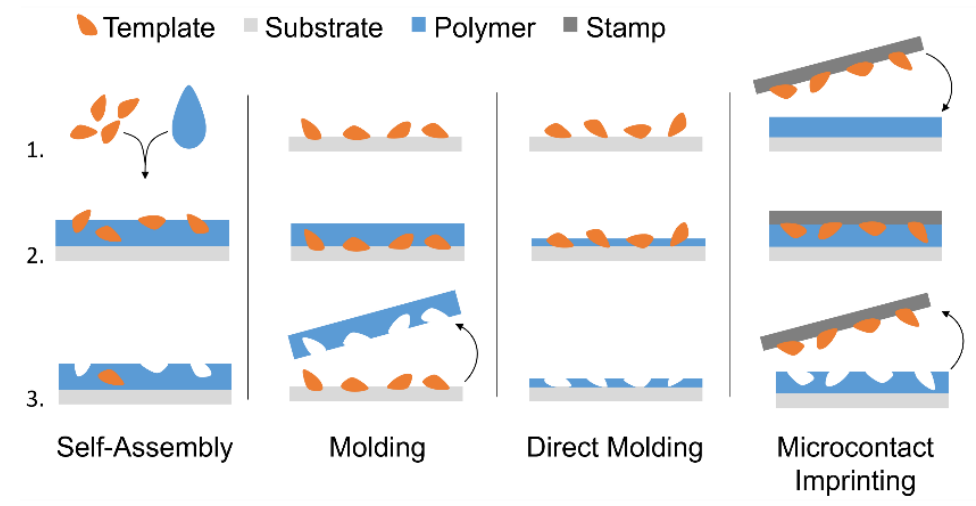

Figure 5-Different methods of SIP manufacturing

\subsubsection{The Transducer}

The next big part of a biosensor, as well as the next step in the sensing process after the analyte binding, is the transducer element, which uses the binding of the analyte to the recognition layer and translates it into a measurable signal. There are three main categories of transducers: optical, electrochemical and physical, the latter being often mass-based 
[68]. Optical transducers use the characteristics of light such as intensity, wavelength and refraction. Electrochemical sensors on the other hand sense based on characteristics such as electrical current or the resistance thereof. Lastly, physical sensors use physical properties of the analyte such as the mass in quartz-crystal microbalances (QCM) or thermal resistance in the heat-transfer method (HTM).

\subsubsection{Optical Transducers}

\section{Colorimetry}

Colorimetric readout systems are the most common systems due to their use in lateral flow assays such as pregnancy tests. In colorimetric systems a color change is either detected via observation without any instrument, which is the case in most lateral flow assays or via instrumentation, this could for example be a smartphone camera with appropriate software, allowing for a semi-quantitative readout $[69,70]$.

As briefly mentioned before, lateral flow assays work with a nitrocellulose membrane for sample-transport, the liquid sample is added onto a sample-pad and is transported via capillary forces into a conjugation pad, here labelled detectionantibodies bind to the target-analyte (Figure 6 Step 1.). The detection-antibodies are usually tagged with colloidal gold, which turns a clearly visible red color when it gets concentrated on a small area. While gold is the most common tag, it is by far the only one, to improve sensitivity the antibodies can also be tagged for example with enzymatic labels or fluorophores (vide infra) [8]. The analyte, which is now attached to a detection antibody, moves further along the stripe to the test-area (Figure 6 Step 2.). Here the recognition-element is immobilized. If the 
analyte of interest is an antibody, which is often used to test for current and past infections or immunity to infectious disease, the immobilized recognition element is an antigen able to bind the antibody (this corresponds to a normal ELISA assay) (Figure 6 Step 2.a). If the analyte of interest is not an antibody then the recognition element is usually an antibody that is able to bind the target analyte (this corresponds to a sandwichELISA-assay) (Figure 6 Step 2.b). In both cases, the target analyte will bind to the recognition elements and in turn the colloidal gold tagged detection-antibodies will concentrate onto the detection zone, resulting in a clearly visible red line. As a control, a zone after the testing zone is used which presents immobilized antibodies that can bind the detection antibodies and indicated a proper fluid flow through the device (Figure 6 Step 3.) $[8,71-75]$. 

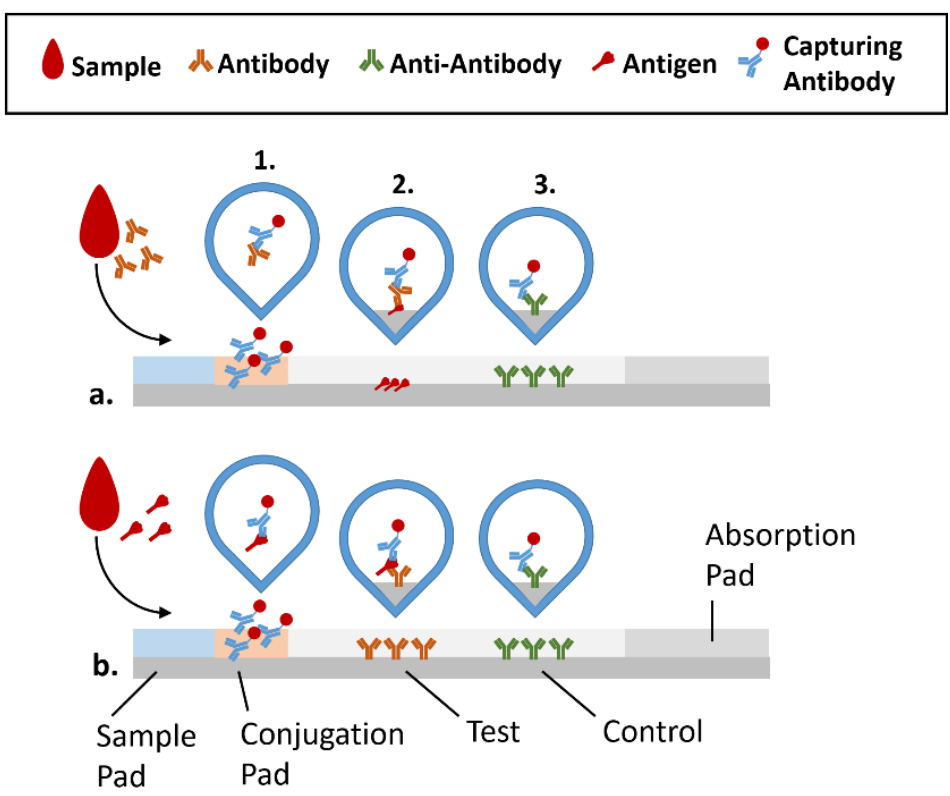

Figure 6 - A lateral flow assay in normal (a.) and sandwich configuration (b.). The target analyte gets transported through the device by capillary forces in the nitro-cellulose membrane. 1. Target and detection-antibody conjugate at the conjugation pad. 2. Target binds to the recognition element.

3. Detection-antibody binds to the control-recognition-elements.

While lateral flow assays are an example of a colorimetric detection via concentrating of colloidal gold, there are other methods of colorimetric systems, one example is the use of simple reactions which yield a color or enzymes which catalyze a colorless substrate into a dye. This was first used as PoC devices in straightforward dipstick tests. Dipstick tests are widely available for example for urin analysis, the Chemstrip by Roche or the Multistix 10SG by Siemens Healthcare being examples [76]. Here for example glucose can be detected via 
immobilized glucose oxidase and a chromogen. The glucose oxidase (GO) oxidizes the glucose to gluconolactone and hydrogen peroxide, the latter then drives the reaction of the chromogen with horse-radish peroxidase (HRP) to water and a visible dye [76] as shown in Figure 7. Protein detection can be achieved via tetrabromphenole blue which turns from yellow to blue in presence of protein due to the formation of a protein dye complex $[76,77]$. White blood cells can be detected via the esterase contained in granulocytes which catalyze a reaction with pyrrole carbonic acid ester to produce a dye [76]. Several common biochemical assays can be deployed in this way combining them on a paper substrate as a multiplex PoC dipstick tests [78]. While usually they are only qualitative or semi-quantitative, the use of optical sensors, for example in smartphones can help to develop colorimetric PoC tests that can measure in a quantitative fashion [79]. 


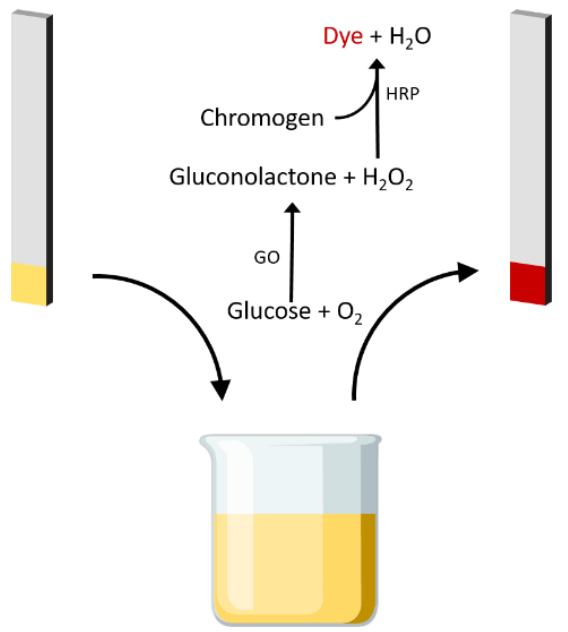

Figure 7 - Example of an dipstick test: Glucose detection via glucose oxidase $(G O)$ and horseradish-peroxidase (HRP) reactions with glucose and a chromogen respectively.

Other applications using a colorimetric readout is the substrate displacement colorimetry, here MIPs for a certain target analyte are created and after removal of the template, the MIPs are loaded with a dye that has a lower affinity to the MIP cavity than the original template. When a solution containing the analyte is brought into contact with the MIPs, the analyte rebinds inside the MIP cavity and in turn replaces the dye. The now freed dye colors the sample liquid, which is visible by bare eye $[80,81]$.

\section{Fluorescence}

Fluorescence is based on fluorophores, which are able to take up and get excited by a photon of a certain wavelength and then in turn emit a photon of a lower wavelength which is used for detection [82]. This can be exploited for biosensing in different ways, for one simple fluorescent tags, for example on 
antibodies, are widely used, not only for biosensing, but to selectively mark certain parts of, for example cells in order to locate certain proteins [83]. Fluorescent based transducers are more complex than the colorimetric or electrochemical sensors but they show benefits when it comes to continuous monitoring or use in difficult matrices and real time analysis [84].

Figure 8 shows different approaches that use fluorescently marked antibodies. All work by binding or replacing fluorophores from the surface. In a sandwich assay the target of interest is captured on the surface by an immobilized antibody and then labeled by a second fluorescently labelled antibody. In turn the fluorescence intensity at the surface increases and unbound antibodies can be washed away [84, 85]. Competitive and displacement assays on the other hand are indirect and can be used for example when the target-analyte is too small to have binding sites for more than one antibody [84]. In an competitive assay a target-analyte is pre-immobilized on the surface, when labelled antibodies as well as a sample is added, the target-analytes inside the sample will compete with the immobilized target for the antibodies and thus fewer antibodies will attach to the immobilized target, which can be monitored as decrease of fluorescent on the surface. Another option is the displacement assay in which a recognitionantibody is immobilized and a labelled target is added to the solution to adhere to the antibody. When a positive sample is added the target from the sample displaces the labeled target, resulting in reduced fluorescence [84]. 


\section{Sandwich Competitive Displacement}

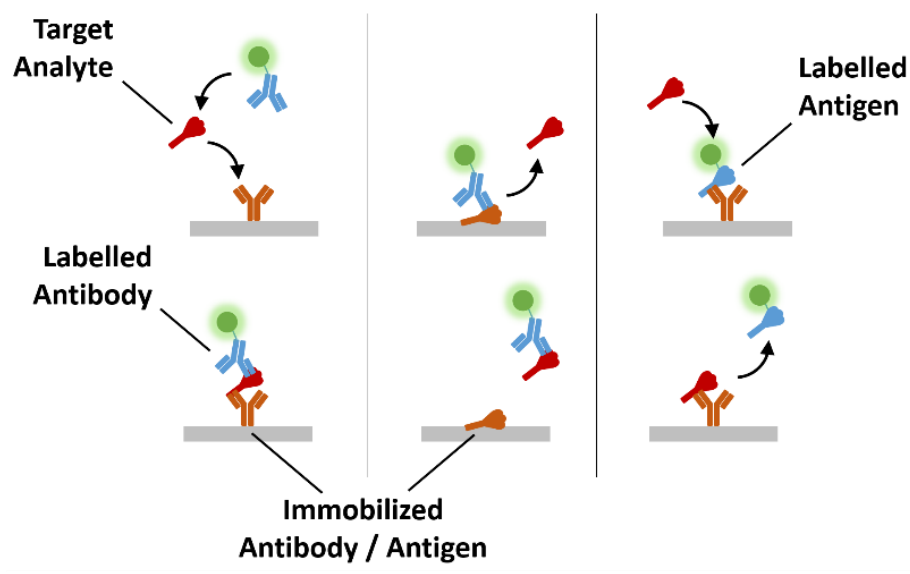

Figure 8 - Different approaches for fluorescent assays.

The assays shown before are relatively simple and are solely based on the attachment or detachment of fluorophores as well as washing, however more complex fluorescence based systems exist. These are commonly used in quantitative polymerase chain reaction (qPCR) $[86,87]$. While qPCR also uses simple dyes that increase their fluorescence when bound to double stranded DNA, like SYBR Green I [86], more complex sensors based on Förster resonance energy transfer (FRET) - fluorescence and - quenching are also available (Figure 9). In FRET, the excitation of a fluorophore is transferred to a second fluorophore in close proximity without the emission of light. This means the first fluorophore (the donor) is quenched and the second (the acceptor) is excited [88-90], it can return into the normal state by either emitting fluorescence itself, or by emission of heat [88]. An example of 
a sensor based on this principle is the common TAQ-MAN probe, a hydrolysis probe in which a fluorophore and a quencher are attached on a short oligonucleotide chain, which hybridizes with the target DNA strand (if present). Due to the proximity of quencher and fluorophore the fluorescence is deactivated. When the elongation phase starts, the Taq DNApolymerase travels along the template strand and uses its 5' 3 ' exonuclease activity to cleave the fluorophore from the quencher, separating both elements and restoring fluorescence in the process $[86,91]$. Molecular beacons have a similar working principle, here fluorophore and quencher are attached to 5'and 3'end of an oligo chain, which forms a hairpin loop when it is not hybridized. This brings fluorophore and quencher in close proximity, prohibiting fluorescence. When the oligo hybridizes with the target DNA it loses the hairpin structure, and thus the distance between fluorophore and quencher increases, restoring fluorescence $[86,87,92]$. This can also be used with two fluorophores and FRET-fluorescence instead of quenching, enabling measurement wavelength at two emission wavelength representing the bound and unbound state [93]. FRET-fluorescence is also used in binary hybridization probes, here the FRET pairs are brought in close proximity to each other by tagging them to oligonucleotides which bind in close proximity at the target strand [87]. While those principles are widely used in applications concerning nucleic acids, the basic principle of FRET-fluorescence and -quenching works with different recognition elements, such as antibodies, as well as long as they are able to bring the FRET partners in close proximity [87]. A large benefit of these techniques is that they can be used with different fluorophores, enabling multiplexing [86]. 


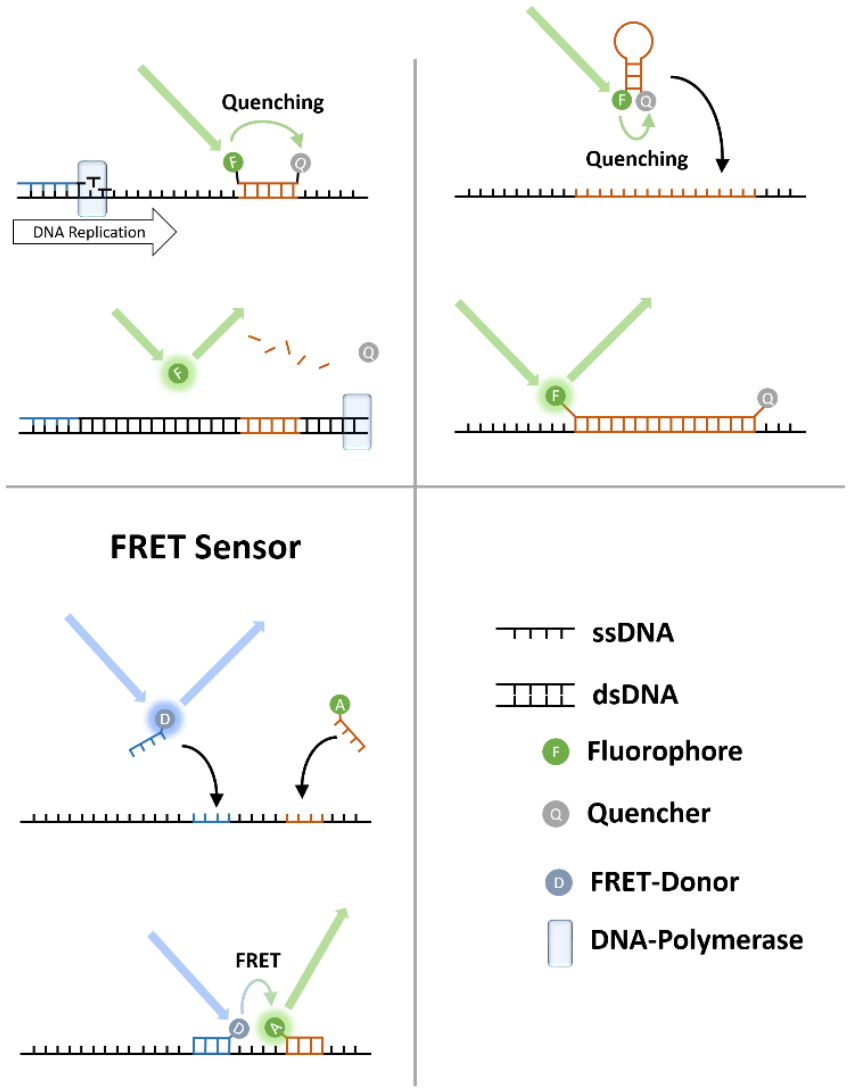

Figure 9 - Different approaches for nucleic acid fluorescent probes

Ribo- and Toehold-Switches

Ribo-switches and toehold-switches are examples of even more complex readout methods using (often, but not necessarily always) fluorescence. Here RNA sequences are used, which 
self-align and form a loop that blocks their ribosome bindingsite. Upon hybridization with the analyte DNA the ribosome binding-site is freed. This can be used with a cell-free expression system, which contains all the necessary enzymes and materials for protein expression. As soon as the ribosome binding-site is freed, the RNA can be translated into a reporterprotein such as green-fluorescent protein (GFP) which can then be used for optical readout. The benefit of using DNA and RNA for diagnostics is that they can be easily amplified, allowing for lower limits of detection [36, 94, 95]. Figure 10 illustrates the process.

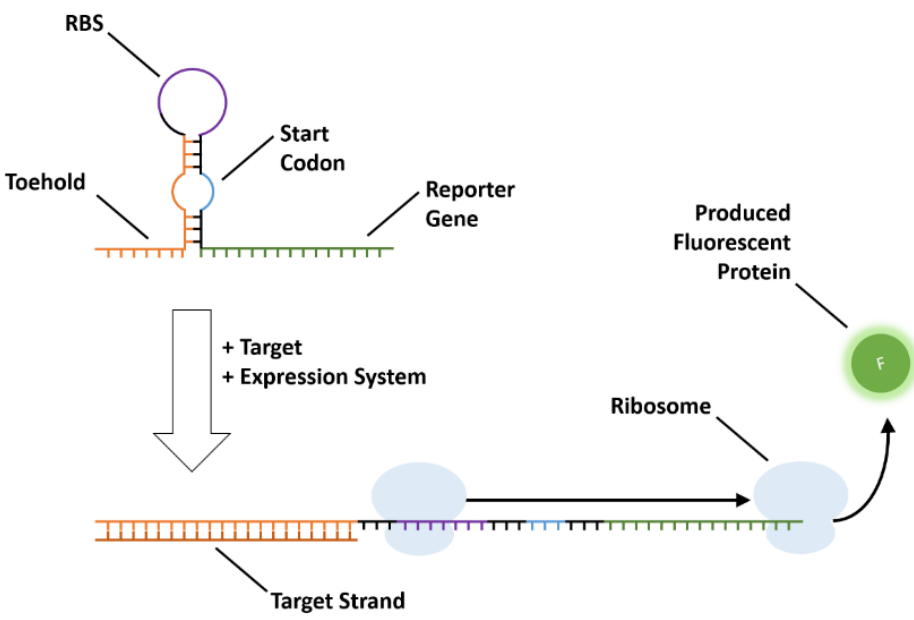

Figure 10 - The working mechanism of a toehold switch 
$S P R$ - Surface plasmon resonsance.

Plasmons are collectively oscillating free electrons in the electron cloud of a metal. A plasmon wave can be initiated by light, which is total-reflected on the surface between a metal and a dielectric. SPR sensors can work in different ways but most often use refraction as a method of readout. The minimum in reflection is found at the excitation angle of the plasmon, due to the energy they take up. This angle changes depending on the mass coupled to the surface, therefore a change in mass leads to a change in refraction which can be measured [96]. As a label free, highly sensitive, real-time method, SPR is a highly important method, especially for analysis of interactions of biomolecules [96, 97].

Localized surface plasmon resonance (LSPR) does not use a gold-film but instead gold nanostructures, which can take different shapes from disks and rings $[96,98,99]$ to spikes and mushroom like structures [100-102]. The readout can be conducted via the wavelength shift in absorbance maxima that happens in response to bound analytes [100, 103]. This shows another benefit of ISPR, light can be used at any angle since the readout works via the absorption instead of the shift in refraction, which results in an easier setup [100, 104, 105]. Figure 11 displays both methods. 


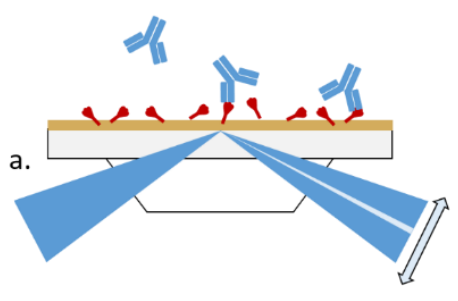

Minimum Refraction Shift

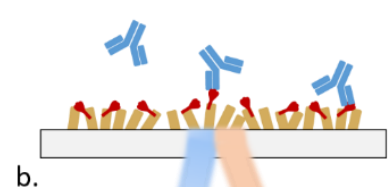

Wavelength Redshift

Figure 11 - SPR and ISPR Methods

\subsubsection{Electrochemical Transducers}

Electrochemical sensors often work with enzymes as recognition elements [106] and are usually equipped with a reference electrode, which maintains a stable potential, a working electrode which is the location of the recognition reaction and lastly a counter electrode which closes the circuit $[106,107]$. When a target binds, depending on sensor system a change in different electrical properties such as potential, current, charge or resistance takes place that can be analyzed $[106,108]$.

\section{Amperometry}

Amperometry based sensors are relatively straightforward electrochemical biosensors, mostly measuring a generated current resulting from a redox-reaction [106, 109]. As briefly mentioned in the beginning, the glucose meter is the poster child of 'high-tech' PoC devices and one of the most important in-vitro diagnostic device in general. Glucose meters use an 
electrochemical amperometric transducer and an example of the occurring reaction is given in Figure 12. Blood is acquired via a lancet and a finger-prick and capillary-force is used to suck the droplet into the strip and transport it to the electrodes. The enzymes catalyze the glucose in the sample and start a signaling cascade leading to an electrical current on the electrodes of the sensor. Enzymes used are most often Glucose Oxygenase and Glucose-dehydrogenase [14, 15, 110, 111].

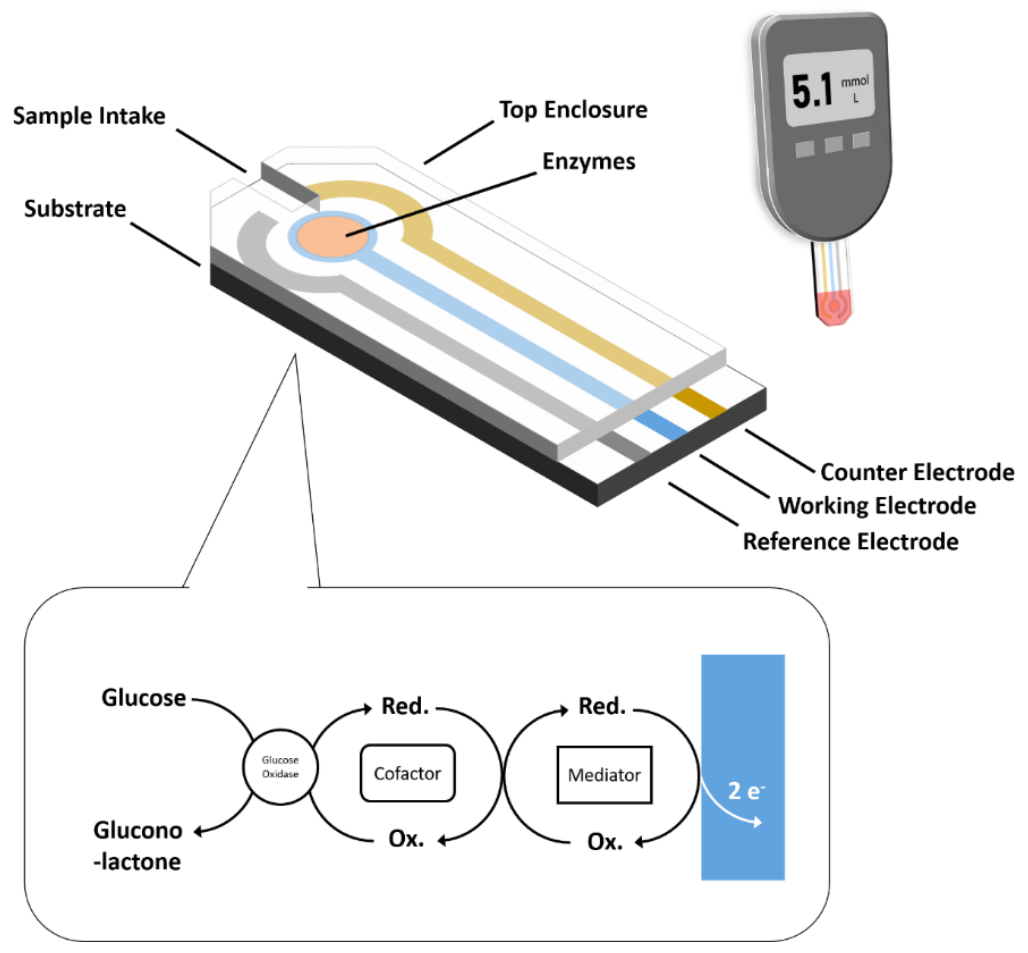

Figure 12 - Setup of a amperometric glucose sensor 
The displayed system is a glucose meter of the second generation. While the enzyme glucose oxidase is oxidizing glucose to gluconolactone it reduces a cofactor (often FAD or PQQ) which then in turn reduces a mediator that hands the electrons to the electrode where the current is measured [112]. Compared to this second generation sensor, the first generation used $\mathrm{H}_{2} \mathrm{O}_{2}$ produced by the cofactor to transfer the electrons to the sensor, which required oxygen. Third generation glucose sensors use the cofactor itself to transfer the electrons to the electrode $[112,113]$. While this is a relative simple example of an amperometric readout, where the voltage is held constant, different variations exist. For example, cyclic voltammetry is similar to normal amperometry as the monitored factor is the electrical current. However contrary to normal amperometry, the voltage is not held constant but is varied. For this the voltage is increased in a fixed rate from a starting voltage. If the electron transfer resistance changes and redox reactions occur, a change in current is visible [96, 114, 115].

\subsubsection{Mass Transducers}

A wide range of transducers can be put under the umbrella term of mass transducers. Usually they are connected to physical properties related to the mass, such as inertia or thermal resistance of the analyte.

Acoustic

Well-known examples of mass-sensitive transducers are quartz-crystal-microbalances, which use piezoelectric quartzcrystals. If an electric current is applied on the crystal, it starts resonating, the resonance frequency is dependent on the mass 
attached to the crystal. If the mass increases, for example by analyte binding on the receptor layer, the resonance frequency decreases in a linear fashion. For usage in liquids and with nonrigid analytes, it is more complex as the viscoelastic properties also have to be accounted for [96, 116]. One of the main benefits of QCM systems are their high dynamic range, enabling their use from small molecules up to cellular targets [96, 117, 118].

Surface Acoustic Waves (SAW) devices are closely related to QCM devices, but instead of using a bulk acoustic wave, like the QCM, they use, like their name already suggests, an acoustic wave on the surface of the device. SAW use interdigital electrodes (ITD) that are built on top of a piezoelectric material and are used as sender and receiver. One electrode converts an electric- into an acoustic wave, which travels to the receiver, which converts the acoustic wave back into an electrical signal. The amplitude as well as the velocity of the wave is dependent on the mass bound on the surface as well as the conductivity of the medium and viscoelastic changes [96, 119]. Figure 13 illustrate both, QCM and SAW devices.
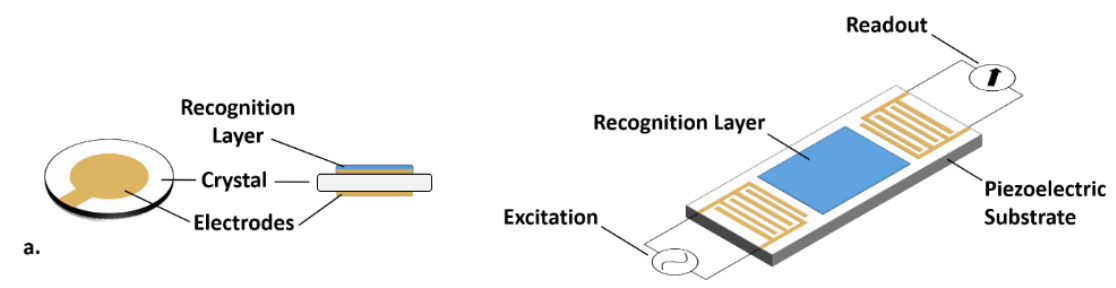

b. 


\section{Heat-Transfer-Method}

The heat-transfer method (HTM) is based on the thermal transfer through the recognition layer (Figure 14) and is the method that will be further investigated in the experimental section of this thesis. In this method, the recognition layer (for example a SIP) is applied on a substrate, which is placed on a copper element. The copper is heated to a temperature $T_{1}$ and held stable using a PID controller. On the receptor layer side, the sample is flushed in and the measuring chamber temperature $T_{2}$ monitored, if target analytes are present in the solution they bind to the recognition elements and thereby changing its thermal resistance [120], which is defined as:

$$
R_{t h}=\frac{T_{1}-T_{2}}{P}
$$

With $T_{1}$ being the temperature of the copper, $T_{2}$ the temperature of the solution above the recognition layer and $\mathrm{P}$ the input power $[120,121]$. This versatile method has been demonstrated with different types of recognition elements. It was used together with ssDNA as recognition element to identify single nucleotide polymorphisms, here the ssDNA is curled up on the surface, after hybridization the formed dsDNA is less flexible and standing up straight, hence it cannot insulate the surface as well and the thermal resistance decreases [35, 122]. Other applications have the opposite effect on the thermal resistance. The HTM can be used together with surface imprinted polymers to measure binding of bacteria [123, 124] or mammalian cells $[65,125,126]$. When they bind on the surface they insulate and in turn increase the thermal resistance, as a result, the temperature in the flow cell drops. An increase in thermal resistance can also be observed in the detection of 
peptides and proteins [127] as well as small molecules [128130] measured via MIP recognition. The heat-transfer method is very affordable as it only takes a heating element and two temperature sensors.

The method was further developed into thermal wave transport analysis (TWTA). In TWTA, the heater is not kept at a determined temperature, but is used to create a thermal wave, which travels through the recognition layer. A binding of a target analyte is consecutively measureable by a shift in both phase as well as amplitude of the measured wave $[63,131]$.

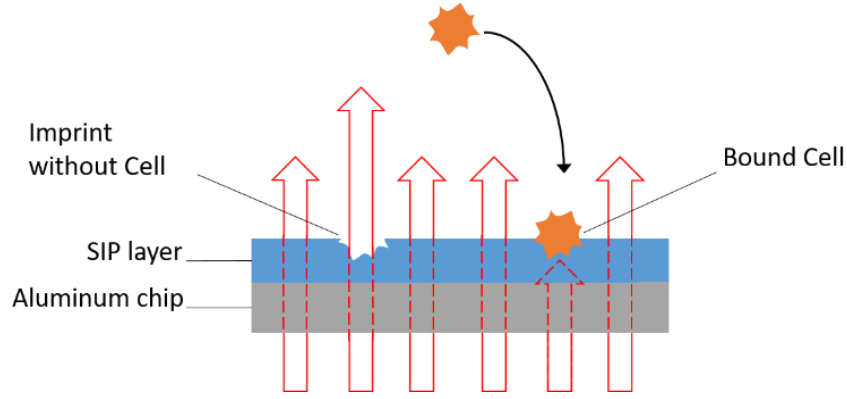

Figure 14- Schematics of the HTM with surface imprinted polymer as a receptor layer

\subsubsection{Sample Management}

Recognition layer, transducer and readout are the elements, which are mostly counted as the main elements of the biosensor. However, sample management is an important part that often is neglected. Especially in PoC devices, the way the sample is processed throughout the device is of critical importance, since it might have to fulfill several sample preparation steps that would have been originally conducted inside a well-equipped laboratory. Here the field of 
microfluidics opens up a valuable toolbox to integrate essential steps directly inside PoC devices. Steps like sample preparation (for example purification), reagent storage, sample processing, mixing, propulsion of the sample to the sensor, waste storage and more are commonly used in microfluidic Lab-on-a-Chip devices (LoaC) [20].

\subsubsection{What is Microfluidics}

Microfluidics concerns itself with very small quantities of liquids in microscopic channels. There is no unified definition of the exact sizes that count as microfluidic and some authors suggest channel sizes between $100 \mathrm{~nm}$ and $100 \mu \mathrm{m}$ [132], others include channels from tens to hundreds of micrometers $[133,134]$ and up to features of less than $1 \mathrm{~mm}$ [135]. The field started out as an offshoot of microelectronic manufacturing using the processes developed for the creation of transistors and using it to create small microfluidic channels instead [136]. The possibilities of devices with microscopic features became soon apparent and miniaturized total analysis systems ( $\mu$ TAS) were suggested as device that can handle all aspects of the analytic process, from sampling, sample transport and preparation steps up to the detection of analytes in one miniaturized device [136, 137]. Since then microfluidics has come a long way and microfluidic circuits are used to perform a wide array of operations, from reactions in micro reactors [133, 138, 139], over analytical processes in Lab-on-a-chip devices [133, 140] to the reconstruction of human physiology in Organ-on-a-Chip systems $[133,141,142]$. All these areas benefit from the peculiar physical properties that fluids adapt when they are manipulated on the microscale. 


\subsubsection{The Physics of Microfluidics}

Several key factors differentiate the physics of fluids in the microscale from its properties in the macroscale. First of all, fluids move in a laminar flow regime in which no convective mixing takes place, as fluids flow predictably next to each other $[136,143]$. Furthermore, gravity affects fluid less, while surface tension and interfacial tension start to play a leading role [136]. Due to the small scales of channels and fluids, diffusion times are drastically reduced and the surface to volume ratio is drastically increased leading to faster reactions $[136,144]$.

\section{Reynolds Number}

For the physical properties of fluids in microfluidic devices several numbers are important. The first is the Reynolds number, which is defined as following [145]:

$$
R e=\frac{p v L}{\mu}
$$

Whereas $p$ describes the density of the fluid, $v$ its velocity and $\mathrm{L}$ the characteristic linear dimension of the system, lastly $\mu$ is the dynamic viscosity. Therefore, a reduction of the dimension of the channels leads to a reduction of the Reynolds number. If $\operatorname{Re}<2000$ the flow is characterized as laminar for $\mathrm{Re}>2000$ the flow is considered turbulent [136]. The laminar flow regime enables microfluidic systems to conduct several different fluid manipulation methods that are not possible in a turbulent regime. For example, given that liquids do not mix turbulently in the laminar flow regime, sample preparation and purification can be performed by running two aqueous solutions in parallel next to each other, which allows for diffusion between the separate streams [146]. The laminar flow regime is also used to 
separate biological cells, enabling technologies such as fluorescent cell sorting and hydrodynamic focusing [147]. Droplet based microfluidics use the laminar flow to manipulate oil droplets in water or water droplets in oil, in microchannel. These droplets can then be used as micro-reactors, allowing massive multiplexing of experiments [148].

\section{Péclet Number}

The Péclet Number is the second important number in microfluidics, and is defined as follows:

$$
P \text { é }=\frac{L v}{D}
$$

Whereas $\mathrm{L}$ describes the length, $\mathrm{v}$ the velocity and $\mathrm{D}$ the diffusion coefficient of the particle. The Péclet number can also be applied for heat diffusion in which $\mathrm{D}$ is exchanged with the thermal diffusivity 'a'. The number describes the ratio of advection rate to diffusion rate $[149,150]$.

Schmidt Number

Finally, the Schmidt number can be derived from the Péclet and Reynolds number and is defined as follows:

$$
S c=\frac{P e}{R e}
$$

The number therefore describes the relationship between moment diffusivity and mass diffusivity [151].

\subsubsection{Manufacturing Methods}

Soft Lithography

Soft lithography or replica molding, is the most widespread methods for microfluidic manufacturing in laboratories [135, 
152-155]. In this method, a master mold is fabricated, usually via photolithography as illustrated in Figure 15. A viscousliquid photoresist, most commonly SU-8, is spin-coated onto a substrate, usually silicon. The spin-coating enables the finetuning of the SU-8 layer height, which is dependent on the speed [156]. In the next step, a photo-mask is used to expose the SU-8 to UV-light. SU-8 is a negative photoresist, meaning the SU-8 that is exposed to UV-light cures and becomes solid while unexposed parts stay liquid and can be washed away during development. The photo-mask therefore is translucent in places where later microfluidic channels are wanted. After curing, the uncured photoresist is dissolved, and only the cured channel structure with its defined height remains.

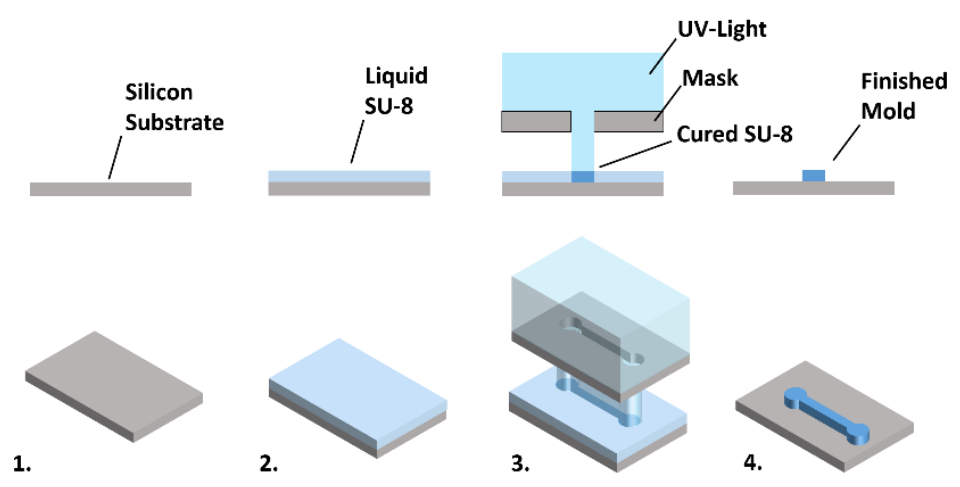

Figure 15 - Photolithographic manufacturing of the master mold: 1. The silicon substrate. 2. SU-8 spin coated on top of the substrate. 3. The SU-8 gets exposed to UV-Light through a mask to cure it 4. The uncured $S U-8$ is washed away. 
After fabrication of the mold, it can be used to construct microfluidic channels via soft lithography (Figure 16) [153$155,157,158]$. For this polydimethylsiloxane (PDMS) (mostly Sylgard 184) is mixed with its curing agent. The normal mixing ratio is $1: 10$, but for softer more elastic PDMS structures that are used, for example, in quake valves, a ratio of 1:20 is common as well [159]. After mixing the PDMS is placed in a desiccator under vacuum to extract air-bubbles trapped in the viscous liquid during mixing. When the air-bubbles are expelled the PDMS is poured over the mold and set in the oven to cure, which takes, depending on temperature from 30 minutes to 3 hours. Afterwards the cured PDMS can be demolded from the master mold and attached to a different substrate, closing the open side and creating the channel. Bonding is usually done via plasma-bonding, here the PDMS and glass substrate to which it will be bonded are treated with an oxygen plasma, which activates the surface with reactive oxygen groups that are then able to form covalent bonds via a condensation reaction of $\mathrm{SiOH}$ groups to $\mathrm{Si}-\mathrm{O}-\mathrm{Si}$ bonds [154], with each other, attaching the substrate and PDMS. [153-155] 

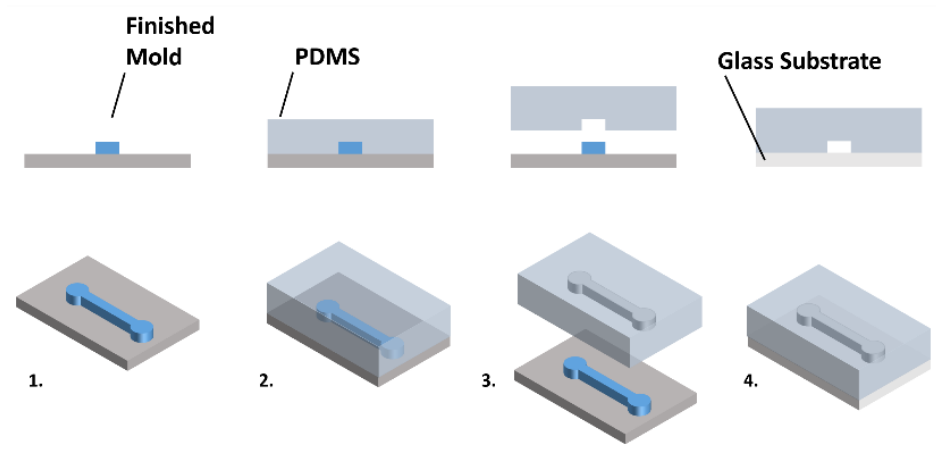

Figure 16 - Soft lithography of the microfluidic channels: The finished mold is used (1.) and covered with liquid PDMS (2.), which is consecutively cured taking on the shape of the mold (3.). The PDMS is then plasma-bonded to a substrate to seal the channels (4.).

PDMS has ideal properties for microfluidic devices and therefore it is not surprising that it is one of the most used materials in microfluidics [136, 160]. The material is cheap and easy to mold, it is optically transparent and gas permeable as well as biocompatible $[154,161]$. As a result, PDMS is an ideal material for biomedical research, such as cell-culture and biochemical assays [133, 161, 162]. Due to its elasticity the fabrication of integrated pumps and valves is possible [159, 163]. Exemplary of this is the quake valve, which consists of a control-channel perpendicular above or below of the fluid carrying channel. Application of air pressure in the controlchannel will deform it, leading to a bulge that blocks the fluid channel [159]. Such valves can be placed next to each other and activated in succession to create a peristaltic pump [159]. Another example for a valve and pumping system that uses the elasticity of PDMS is the adaption of a braille display, where 
the different pins that normally display the braille letters are used for fluid control [164].

However, while exemplary in biomedical applications, PDMS is not a great choice for applications in synthesis due to its incompatibility with many organic solvents that can cause PDMS swelling [135, 161]. Another problem with PDMS soft lithography is that the method, while benefiting from rapid prototyping capabilities [160], has major scale-up difficulties compared with the other methods like injection molding and hot embossing which will therefore be discussed below.

\section{Injection molding and hot embossing}

Injection molding is a fabrication technique not solely used for microfluidics but also for mass-production of plastic parts in general [165-167]. The easy scale-up is one of the main benefits of the technology and lead to its commercial success in mass-manufacturing [135]. The method uses a heated (molten) thermoplastic, which is injected into a mold with the required shape. In case of microfluidics, the mold displays the channel structure (as with all molding techniques as a negative, channels are displayed as extrusions). The closed mold is assembled and molten thermoplastic injected under pressure, after cooling, the mold can be opened and the microfluidic structure demolded [133, 135, 165-168]. The method is restricted to thermoplastics, but a wide range of materials are available that match important microfluidic characteristics such as transparency and biocompatibility.

Hot embossing is a somewhat similar alternative to injection molding in which the mold is heated and a thermoplastic substrate pressed against it, instead of having an already molten thermoplastic that gets injected into a mold. When this happens, the heated mold embosses the microfluidic structure 
into the thermoplastic. Comparable to injection molding, hot embossing has its strength in the scalability of the method, and therefore found wide application in commercial microfluidic manufacturing $[135,169]$. Both methods are demonstrated in Figure 17. Hot-embossing can be up-scaled further to hot-roller embossing, in which the mold is a cylindrical roll that imprints consecutively on a long film in a roll-to-roll manufacturing process [170]. One of the biggest downsides of both techniques is the mold fabrication, which is expensive and time consuming and thus only feasible for a high number of produced parts, which excludes the method for fast prototyping, which is the specialty of PDMS soft lithography [135, 171].

Sealing the surface, and therefore creating the closed channels from the open surface-facing channels, is an essential and often problematic step in microfluidic manufacturing [172]. In techniques like injection molding or hot embossing, this can be achieved by adding a second thermoplastic sheet and bonding it via, for example, ultrasonic welding or thermo-bonding [135, 172]. This is easily achieved if the substrate has a flat surface, however for molded parts with topographical surfaces, this can be problematic and a second molded piece fitting the geometry of the substrate has to be fabricated. This can be avoided by the use of topographical vacuum sealing as introduced in Chapter 6. 

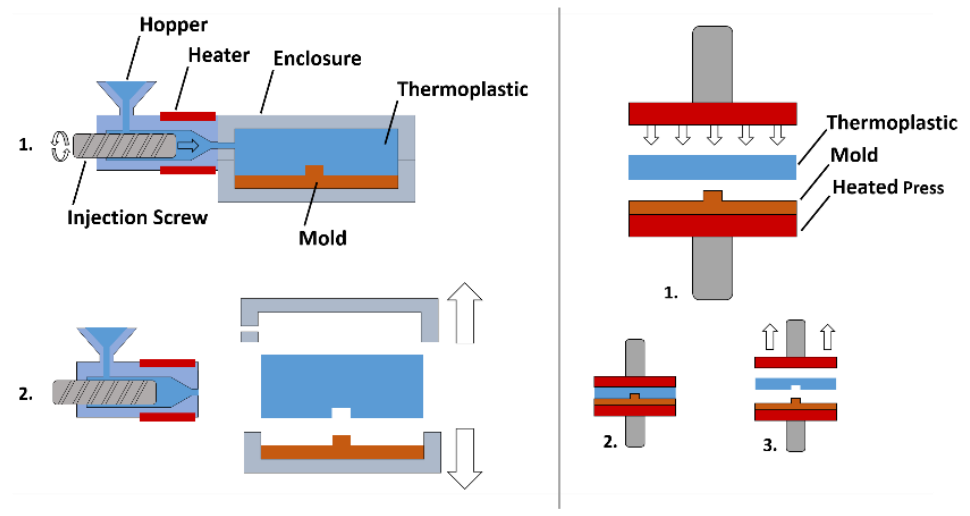

Figure 17 - Schematics of injection molding (left) and hot embossing (right) for microfluidic fabrication

\section{Laminate}

Another straightforward and cleanroom-free way to fabricate microfluidic devices is lamination and lab-on-a-foil technology. Here the microfluidic channels are cut into a thin substrate which is then bonded to other foils which seal the top and bottom [135], as is exemplified in Figure 18. While the cut layer is mostly made out of plastic or tape, the outer bonding layers can also be constructed out of glass [173]. Laminate microfluidic devices present several benefits, the construction process is one of the easiest in microfluidics, as a simple xerography knife-cutter [157] or laser cutter [174] can be used to cut channels inside the foil.

Often used layers are glass as cover layers, adhesive tapes - due to the inherent bonding, and polymers such as polycarbonate and PMMA [135, 157]. The used materials are transparent adding another benefit to laminated microfluidics. To bond the layers adhesives can be used, which is beneficial as the fluid 
layer can already be cut in adhesive tape or transfer tape [157], making the fabrication process easier. However, the use of adhesives as bonding method can lead to uneven layer heights and limited pressure resistance as well as a possible interference of the adhesive $[135,175]$. If thermoplastics where used, thermo-bonding can be used to fuse the layers together [135]. As the other fabrication methods of injection molding and hot embossing, lamination is also very scalable [135].

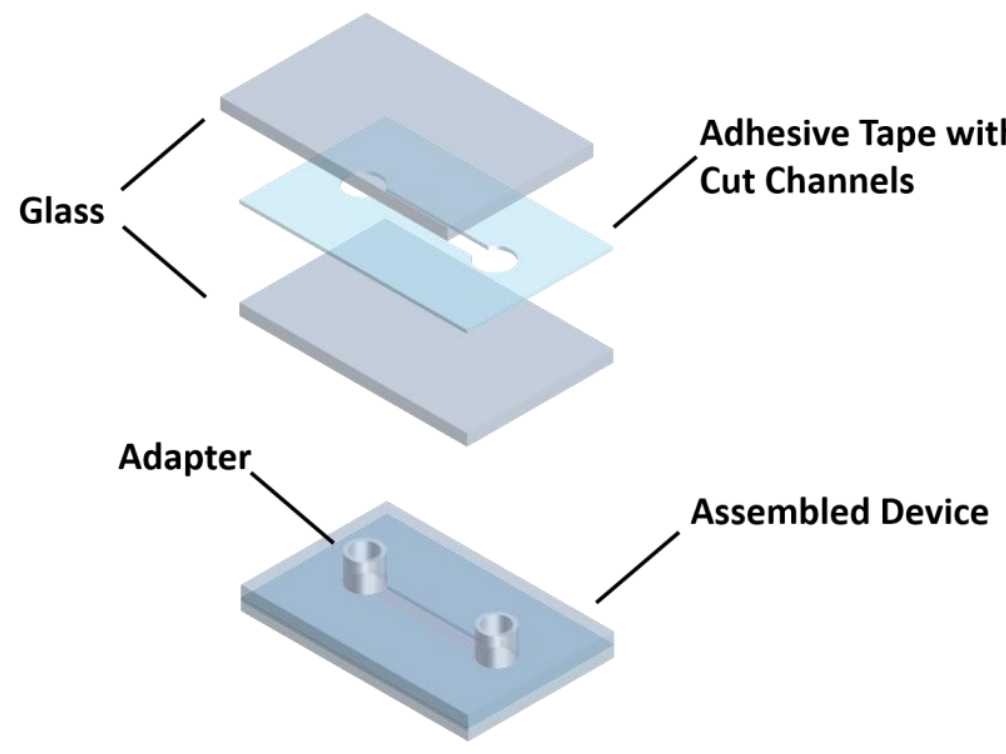

Figure 18 - Laminated microfluidic schematic.

$3 D$ printing

3D printing has become increasingly common during the last ten years and is starting to replace other microfluidic 
manufacturing methods due to its ease of use, low investment costs and cleanroom free fabrication capabilities [157, 176178], these benefits however come with the downside of a lower resolution than traditional methods [178]. A more indepth introduction to $3 \mathrm{D}$ printing of microfluidics will be covered in chapters four, five and six.

While there are many different 3D printing methods, from powder-bed printing to inkjet printers and laser sintering, the most common forms are fused deposition modelling (FDM) and stereolithographic printing (SLA).

Fused Deposition Modelling

FDM printing is the most straightforward variant of $3 \mathrm{D}$ printing. Here a thermoplastic filament is fed into a heated nozzle and molten. The nozzle is able to travel in all three directions $(\mathrm{x}, \mathrm{y}, \mathrm{z})$ in respect to a buildplate, as illustrated in Figure 19. While moving the nozzle in $\mathrm{x}$ and $\mathrm{y}$ direction over the buildplate it 'draws' a layer with the molten plastic, which then becomes solid again [179, 180]. After drawing one layer, it moves in the $\mathrm{z}$ direction onto the next layer and repeats the process. A large benefit in this regard is that almost any thermoplastic can be used for FDM printing [176]. 


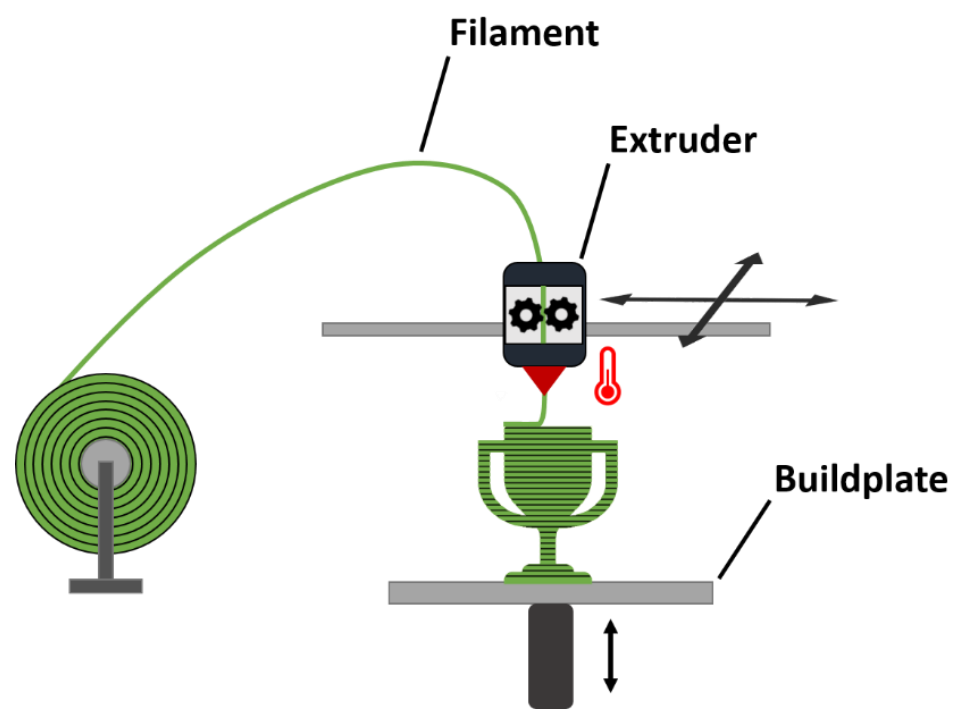

Figure 19 - Schematic of a FDM Printer

SLA Printing

SLA printing is based on liquid photopolymer contained in a vat. The photopolymer can be cured by exposition to UV light. The buildplate is lowered into the vat until it is positioned close to a UV-transparent window. Here, the first layer is exposed to the UV-light and thus cured. The buildplate is elevated to allow resin to flow in-between buildplate and window again. Then the process continues, the buildplate is being lowered again and the next layer cured, this process is repeated layer by layer until the complete object is formed [176, 178, 179]. Different systems for UV-exposition exist, the most common are LaserSLA systems and masked-SLA (mSLA) systems. The Laser system use a UV-laser for exposition of the polymer by 'drawing' the layer onto the buildplate similar to and FDM 
printer [176, 178, 179]. Masked-SLA systems on the other hand use a UV-Light source and a digital mask, for example a LCD screen or a DLP display, which blocks or allows exposure with its pixels in a similar way of normal computer screens and projectors [176, 178, 179]. Figure 20 shows a schematic of the different systems.
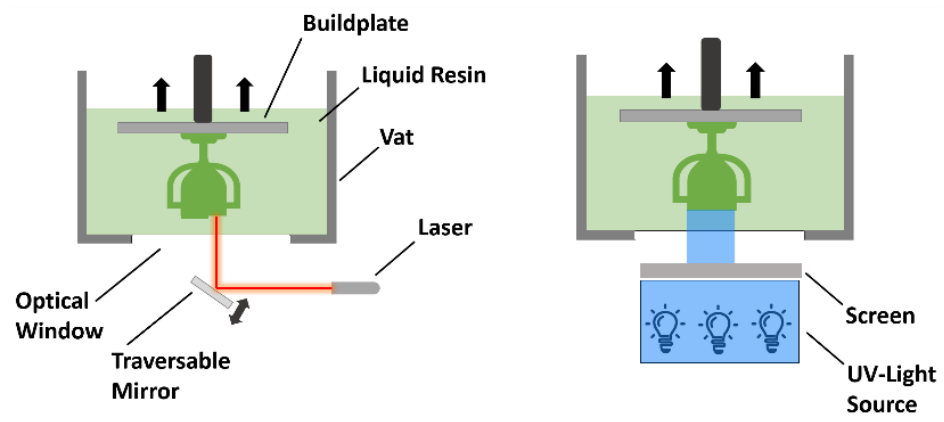

Figure 20 - Resin printing methods: SLA (left) and LCD (right)

\subsection{Aim}

The aim of this thesis is to discuss and answer several aspects of PoC testing. From its fabrication using novel microfluidic fabrication methods over the proof-of-application of novel biosensor principles to the application in the field, a wide array of concepts will be discussed.

The discussion is started by analyzing the actual usage of PoC in low-income countries. This is an elemental question as new developments in the laboratory are plentiful; their actual translation into the field however is rare. It will be discussed which factors contribute to the actual usage of PoC diagnostics 
and which hinder it. This will allow to assess the topic from a different viewpoint which will inform design decisions for future PoC applications due to the unique insights gathered. Thereafter a proof of application of the heat-transfer method as an affordable biosensor system is presented. The method is affordable and easy to use and therefore ideal for PoC use. In addition, its applicability for urinary tract infections will be demonstrated with samples from several test persons. Sample transport through the device and to the sensor is an essential part of a PoC device. Hence, the manufacturing of microfluidics using 3D printing will be covered in considerable detail and applications resulting from this research shown. Finally, topographical vacuum sealing is introduced as a new method that improves on conventional microfluidic 3D printing. With this, the thesis aims to give a birds-eye view on $\mathrm{PoC}$ and PoC devices. From the current usage of PoC in lowincome countries, over the proof-of-application of a novel biosensor system, to novel methods of microfluidic manufacturing for sample handling. Figure 21 shows the specific chapters and their application in the context of a biosensor. 


\section{Introduction}
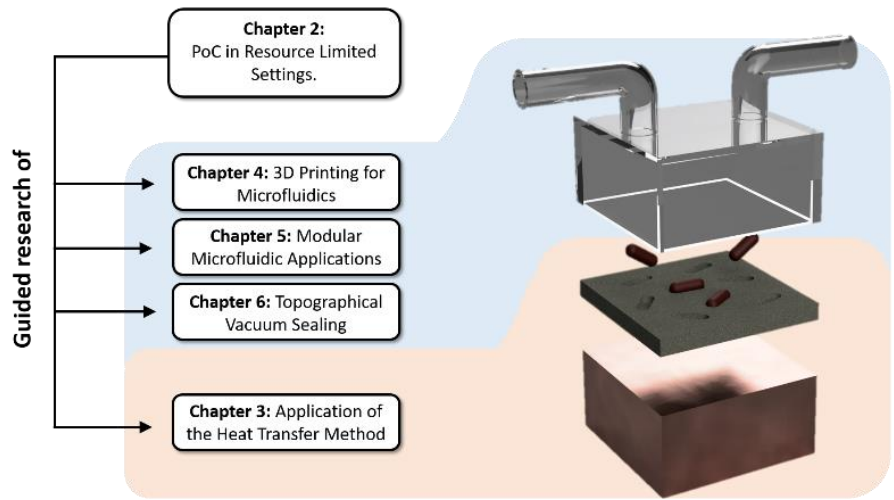

Figure 21 - Overview about the different chapters and their connection to the specific parts of the biosensor. 


\section{Point of Care Diagnostics in Resource-Limited Settings}

A Review of the Present and Future of PoC in Its Most Needed Environment

Adapted from:

B. Heidt, W. F. Siqueira, K. Eersels, H. Diliën, B. van Grinsven, R. T. Fujiwara, T. J. Cleij, Biosensors. 2020 10, DOI: 10.3390/bios10100133. 


\subsection{Chapter Context}

This chapter will give an analytical overview of the actual use of PoC devices in Low-Income countries (LIC). PoC use can be especially helpful to healthcare in LIC, as the devices are cheap and do not require any infrastructure. Yet, the vast developments made inside labs just rarely find widespread applications in the field. The factors preventing $\mathrm{PoC}$ diagnostics from entering healthcare systems will be discussed here, to lay a groundwork for the consecutive chapters of this thesis. To this end, PoC devices will be discussed in the wider context of their application in society, since an extensive analysis of the current state of $\mathrm{PoC}$ is necessary in order to uncover the important design aspects of the devices that enable their actual use in the field. This will be helpful for future design considerations, as the entire socio-economic context has to be taken into account while designing a PoC device for a specific environment. The current assumption of the WHO angle of ASSURED (Affordable, Sensitive, Specific, Userfriendly, Rapid and robust, Equipment-free, Deliverable) as a necessity will be challenged and new insights drawn, as PoC devices are discussed from a different and original viewpoint than the usual angle based solely on device characteristics. This will place the later, more technical chapters, in a wider theoretical context.

\subsection{Introduction}

Low- and middle-income countries (LIC/MICs) face severe challenges due to limited economic opportunities. In addition to the economic struggles, LICs also bear a large burden of transmittable diseases, posing severe risks to the population's wellbeing [1]. Healthcare systems and healthcare providers in 
LICs are often ill-equipped to treat the patients in the best possible way, especially in rural areas. Given the economical and infrastructural challenges in LIC, PoC diagnostics, which are often characterized by being independent of laboratory or medical infrastructure, as well as being highly affordable and holding considerable promise to improve the situation. Yet the actual commercialization of PoC diagnostic-tests lags well behind the innovative research and developments done in laboratories.

Due to this strange dichotomy between promising, innovative research and very limited valorization into real products, several review articles on the topic have been written in past years. However, most are specialized on one specific aspect. For example, some authors looked in depth at logistical shortcomings [2]; others investigated funding and collaboration considerations [3]. Reviews also summarized the topic in consideration of different viewing angles, such as the technological aspects and their implications, as seen in Figure 22 [4]. Another approach is to distinguish between different usage profiles, from the use at home up to the use in a laboratory, and argued that what point-of-care means depends tremendously on how and where it is used, which is shown in Figure 23[5]. 


\section{Point of Care Diagnostics in Resource-Limited Settings}
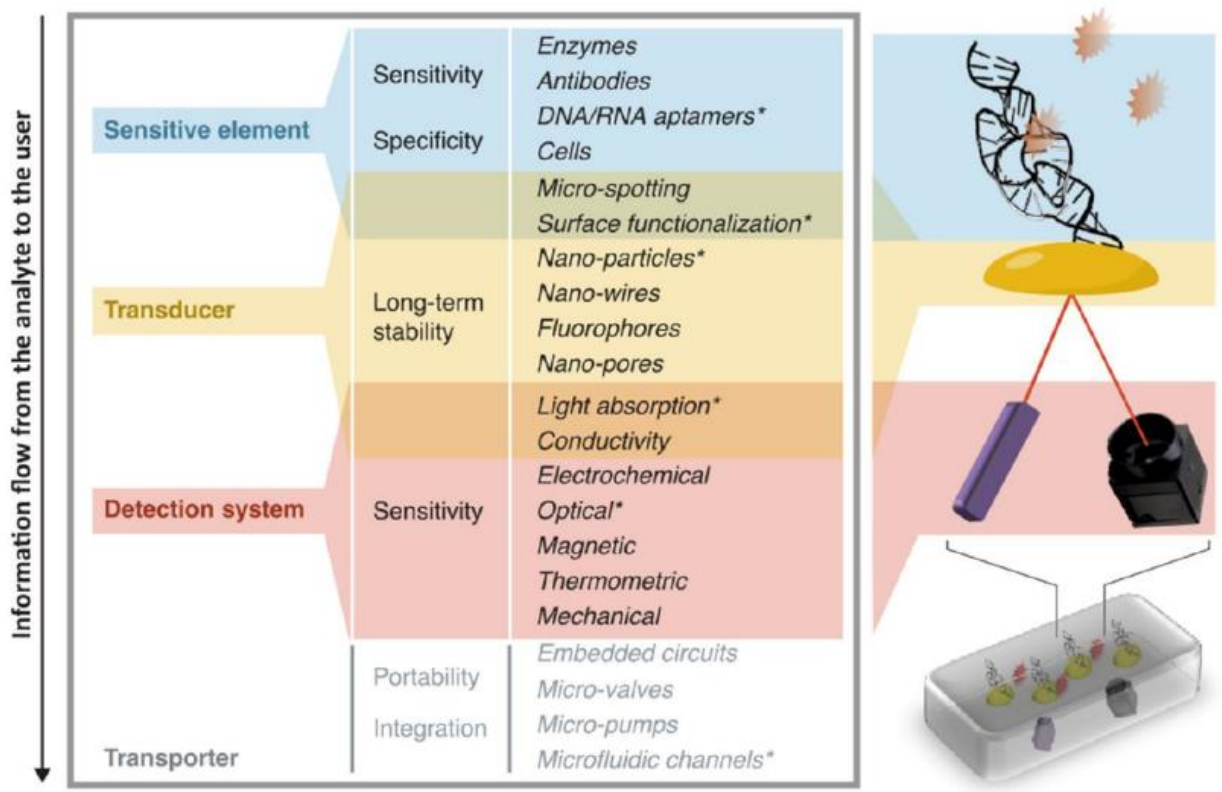

Figure 22 - Migliozzi and Guibentif et al. looked at PoC bottlenecks from a technological standpoint. Figure re-used from [4] with permission under open-access creative commons copyright agreement. 


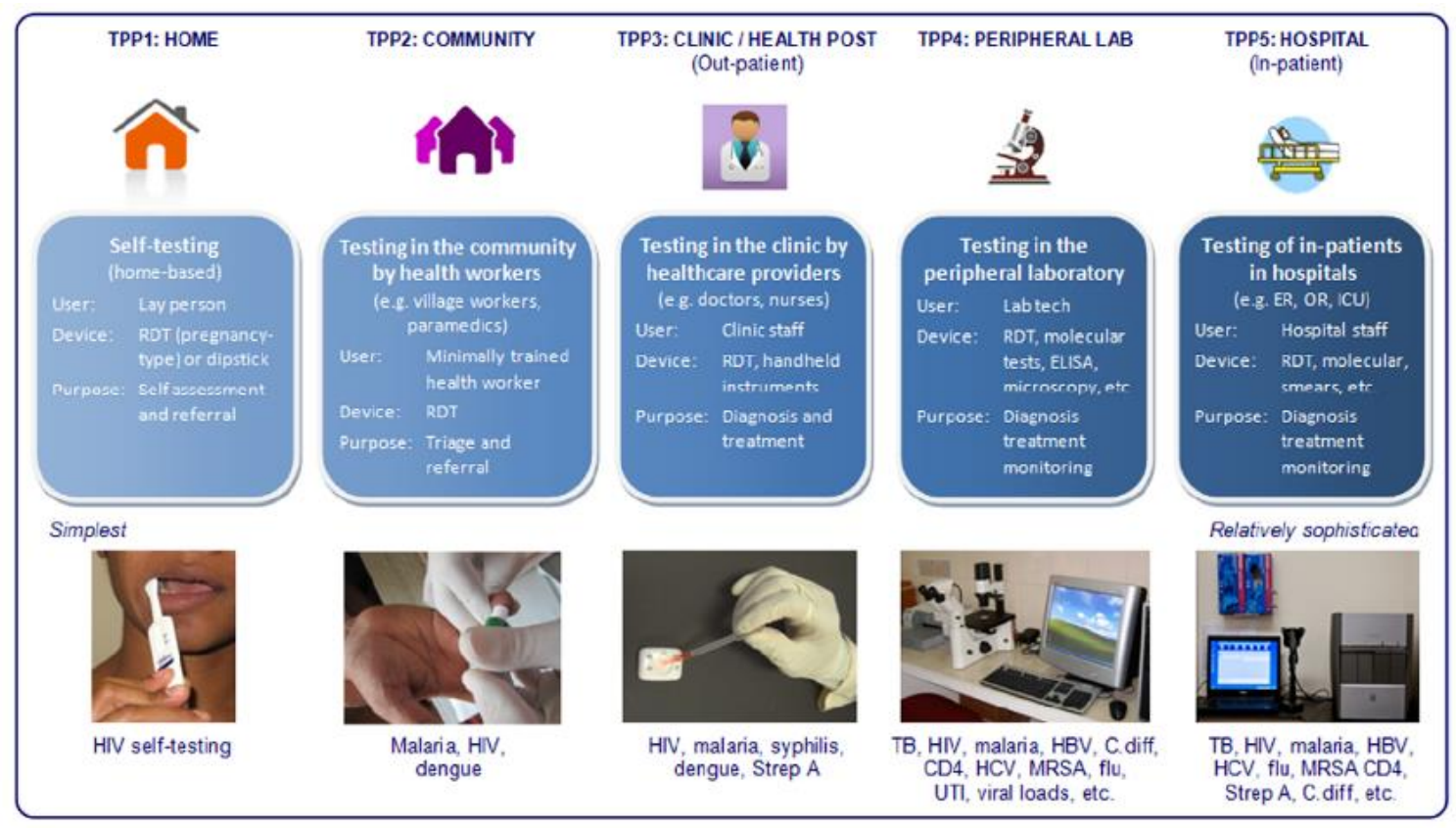

Figure 23 - Pai et al. investigated PoC use within different usage scenarios. Figure adapted from [5] with permission under open-access copyright agreement. 
This review aims to investigate the topic from a different angle. Here, we will look at the barriers of $\mathrm{PoC}$ diagnostics along the entire value chain, from the first idea in a laboratory to the use of the final product by a healthcare provider. With this review we aim to locate the "leaks in the pipeline" of $\mathrm{PoC}$ commercialization, that typically occur when PoC devices do not manage to proceed to a next step in the value chain. Such leaks include problems with funding that prevent the design of a prototype, or intellectual property (IP) considerations preventing market access. In this way, it may be possible to determine at which discrete steps the transition fails and a $\mathrm{PoC}$ device that was maybe once-promising becomes abandoned or underused. For this, the value chain of PoC devices is separated into three distinct domains, which themselves consist of separate steps. These domains are labeled research, market and usage, respectively, and any device has to achieve success in each of the steps within these domains to be able to reach the market and exert a benefit to the patient at the end of the value chain. These different domains in the value chain can be further subdivided into subdomains (Figure 24) with research e.g., consisting of "Fundamental Research" or "Proof of Concepts and Prototyping". In the second part of our review "The Market" is investigated, which looks at fundamental economic problems in the steps "Market Introduction" and "Market Penetration". Finally, the "Usage Environment" will give an insight into the last barriers for actual use by healthcare providers. In this way, we aim to incorporate the entire value chain by examining both original research and review articles. 


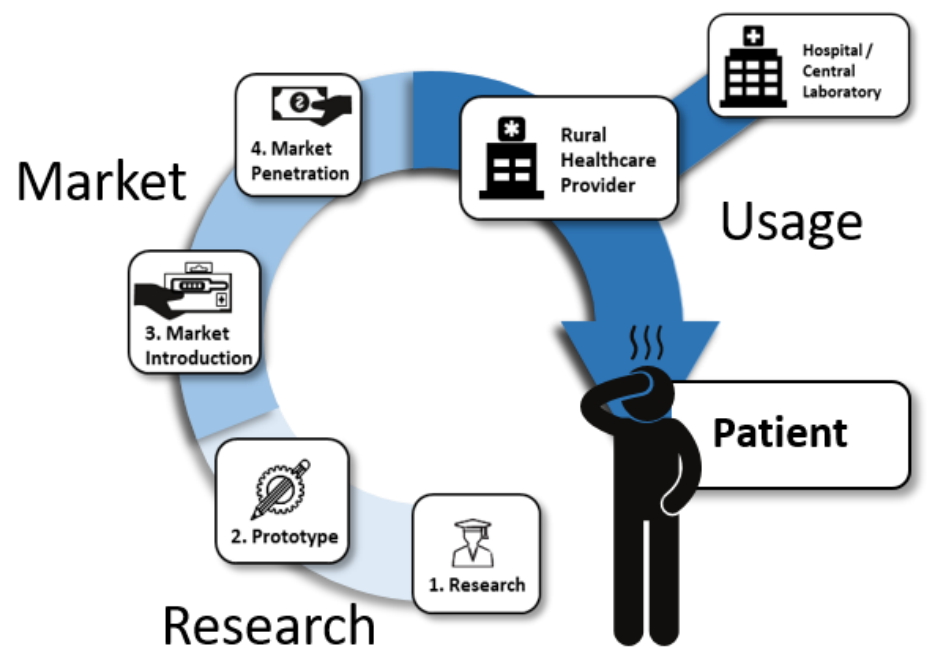

Figure 24 - Segmentation of stages a PoC device hast to pass to be able to bring a benefit to the patient.

\subsection{Research}

2.3.1 Fundamental Research: Funding Availability and Focus

Fundamental research is the first step in the development of a PoC device and concerns all the basic research that is not yet directly related to a prototype or a product. Historically, the research of PoC devices started with simple dipstick tests with immobilized reagents, for example, for the detection of glucose [6]. Later the laboratory use of immunoassays, especially the high sensitivity of radioimmunoassays and enzyme-linked immunoassays, created interest in improving those methods into rapid tests, which eventually became lateral flow immunoassay, the most abundant type of PoC device [7]. While 
lateral flow devices are the most common, the trend in research goes towards devices with higher complexity, which are able to handle more complicated samples, can multiplex and detect challenging analytes [6]. The increased complexity of these devices is shown in their mechanisms of action, which are often optical, electro(chemical) or magnetic [8], but also include other mode of actions, e.g. thermal sensor systems $[9,10]$. Optical systems include more straightforward UV/Vis or fluorescence sensors, as well as more complex systems using quantum dot and surface-plasmon-resonance technology or even genetically encoded biosensors [11-14]. Examples of electro-(chemical) systems range from basic ampere or voltametric systems over graphene-based field-effecttransistors to DNA-annealing-based redox-reporter assays [1518]. However, compared to the abundance of new research, the actual commercialization lags behind and market examples welcoming these new innovations are rare [19].

\subsubsection{Choosing a Suitable Design Philosophy}

There are several factors that may misguide new developments, an important one being the design philosophy of new devices. With respect to this issue, a stark contrast from the research philosophy in high-income countries (HIC) and the needs of LICs is evident. Research in HICs is rarely aimed at inexpensive technologies with wider impact $[4,20]$, which are direly needed in resource-limited settings. Instead, research in HICs tends to focus on high-efficiency devices with even higher complexity, which is not a problem for affluent countries with good infrastructure; however, due to their complexity, such devices are often not usable (or affordable) in LICs [21]. Several studies point out the differences in approach 
between high-complexity $\mathrm{PoC}$ diagnostic test platforms (HCTs), such as the GeneXpert and low-complexity PoC tests (LCTs), such as widely available lateral flow tests-for example, for pregnancy and malaria $[4,20,21]$. While HCTs can conduct more difficult and sensitive diagnostic tests, they are also more complex and thus require more training, maintenance and infrastructure. LCTs, on the other hand, lack sensitivity and diagnostic power, but are highly affordable and low maintenance.

This is not the only example of different design philosophies and approaches. In the design of biosensors in HIC, the most suitable biomarker for a given illness is chosen without much regard for the needed infrastructure. The ability to perform a venipuncture, for example, is a given in HICs, which means tests developed have no strong restrictions for the needed sample size. This can pose a problem for LICs in rural settings without a trained phlebotomist as venipuncture is not possible and the test has to be able to work either with easier to acquire samples, such as sputum or urine, or the much smaller blood sample quantities of a finger prick or heel-stick samples. Heelstick sample sizes are usually under $5 \%$ of the size of venipuncture samples [22]. This illustrates that the research approach for LICs needs to be different. Instead of building a system around the best biomarker, the system needs to be created around the available infrastructure first, which is a considerably different design philosophy [22,23].

\subsubsection{Taking Aim: Proper Target Analytes}

Next to the different design philosophies, the target of research is different in HICs and LICs. Resource-rich settings do not 
focus on research on neglected tropical diseases (NTDs), which is already evident from their name. The burden of NTDs is mostly nonexistent in affluent countries; instead, they predominantly affect the world's poor, and are therefore less interesting for commercial research, as the chance of return of investment is limited. There is little incentive for affluent countries to deal with many NTDs, mainly due to the low impact these diseases have on the population of these countries [24]. This is also visible in drug development where, from 1975 to 2004 , only $1 \%$ of drugs were developed for NTDs (21 out of 1556) [3].

Other gaps in knowledge might be easier to miss. For example, local differences exist in diseases, such as geographical variability in antigen presentation and DNA/RNA signals. In addition, other diseases prevalent in LICs can have an effect on a target analyte. For example, immunosuppression due to HIV leads to lower host response signals and reduces the sensitivity of some nucleic acid tests for the detection of pulmonary TB $[25,26]$. Another report remarks that, even when there is willingness, funding and know-how about adequate research and design philosophy, there are practical problems as simple as not being able to acquire appropriate samples for assay development [26]. In general, research can only make an impact in LICs if a bottom-up approach is used that takes the infrastructure and environment of LICs into account from the start [4].

\subsubsection{Funding in LICS}

Research in LICs themselves is beneficial as it focuses directly on the regional circumstances and problems, with a LICs- 
centered philosophy. However, PoC funding in LICs themselves is highly inconsistent [20,27]; this leads to dependence on other funding opportunities, such as NGOs and development partnerships. This is not only the case for funding of PoC research, but also for treatment-research and treatment itself. For some countries in Sub-Saharan Africa, HIV expenditures are strongly reliant on external sources, despite this being one of the region's most important health risks. Kenya and Uganda contribute less than $15 \%$ of funding to their own national HIV-relief efforts, Mozambique even contributes only $3 \%[20,27]$. In cases where there is secure funding through outside sources, it naturally tends to focus on treatment or prevention e.g., vaccine development while diagnostic research receives much less funding, leading to an over-reliance on clinical symptoms due to a lack of adequate diagnostics $[4,28]$.

\subsubsection{Incentives to Change Focus in HICs}

Possible solutions are new programs that are being implemented to incentivize product development for neglected tropical diseases [3]. However, such programs are mostly aimed at drug development and include, amongst others, the "Priority Review Voucher (PRV)" program in the United States, which gives out transferable priority FDA-review vouchers. It is one example of an implemented "pullmechanism" to motivate development in neglected areas $[3,29,30]$. Next to such pull mechanisms, "push-incentives", such as the Global Health Innovative Technology Fund (GHIT), a partnership that connects the Japanese Government, several NGOs, as well as large drug and diagnostic manufacturers, and targets poverty-connected diseases and NTDs [3,31]. Other specifically named initiatives are the 
Point of Care Diagnostics in Resource-Limited Settings

"Global Health Investment Fund (GHIF)" and the "Wellcome Trust Pathfinder Award" [3]. Incentive programs such as this may have a large effect when used specifically to incentivize PoC diagnostics.

NGOs e.g., the Bill \& Melinda Gates Foundation have contributed a substantial amount of funding for NTDs research and play an important part in integrating into such programs $[32,33,34,35,36]$. Having large industrial PoC developers on board may not only improve the device situation but may have further benefits in implementing the devices from a market penetration standpoint (vide infra). An increased cooperation due to the mentioned pull mechanisms can also help to prevent a "HIC-Bubble" when stakeholders from LICs are involved.

To change the philosophy of research, "Frugal Development" must be considered from the start to build devices with relatively low complexity that are not only affordable and usable in LICs settings but also maintainable and repairable $[37,38,39,40]$. This not only serves as a base requirement, but also makes several aspects, such as acquiring spare parts and stock management, more straightforward down the line. Additionally, 3D printing and other new technologies might be of use to create spare parts on demand, as those new devices have already been shown to be able to create simple microfluidic parts, and were able to supply hospitals with respirator valves during the COVID-19 pandemic [41,42]. 


\subsubsection{Proof-of-Concept and Prototypes: The Importance of Appropriate Device Characteristics.}

\subsubsection{Device Characteristics}

Engineers in affluent countries tend to design devices that assume HICs infrastructure standards, meaning well-funded laboratories in regulated environments with quality control. This can be problematic in LIC, and therefore special design considerations must be taken into account [20]. Devices that perform well in controlled settings, in which the prototype is tested, often fail when challenged with tropical conditions in LICs [26]. Especially in rural settings, where access to, for example, electricity, might be problematic in terms of powered PoC devices [22,26,43], but also a lack of cold storage options, can form a significant challenge $[9,22,23,43]$. Limited refrigeration and power supply therefore demands that the device and its disposables have to be stable in the long term, even at high temperatures, while powered PoC devices need to be able to run on battery or solar $[4,26,44]$.

\subsubsection{Being ASSURED}

Many authors, agree that the device characteristics that are beneficial are following the WHO guidelines for $\mathrm{PoC}$ diagnostics, which are symbolized by the acronym, ASSURED; which stands for Affordable, Sensitive, Specific, User-friendly, Rapid and robust, Equipment-free and Delivered to those who need them. These requirements are regularly mentioned in publications [20,22,45-48], but it also has to be 
noted that these are ideal and strong requirements that only a selected few devices can meet [21].

While progress has been made on PoC tests for syphilis, chlamydia as well as gonococcal infections, there is still not one test available that complies with all ASSURED criteria [46]. Especially good sensitivity and selectivity, which are two of the most important points, get increasingly more difficult to achieve the closer the system is to a "perfect" system with an accuracy of $100 \%[21,22,26]$. In rural LICs settings, a PoC diagnostic tool needs to be small, portable and highly affordable [43]. While many authors put great emphasis on affordability, others take a different position and argue that Zero-Cost is not an important parameter; arguing that reliability and standardization are seen as more crucial, and remark that it might be misguided to assume that "poverty reduces the value individuals place on their well-being" and therefore focus should be put on efficiency and reliability before anything else [28].

\subsubsection{How Necessary Is It to Be ASSURED?}

Many scientists cite ASSURED as a necessity and a large consensus for the ASSURED criteria among healthcare workers in, for instance, Uganda, has been found [49], Pai et al., give another, more pragmatic and contextual viewpoint. ASSURED, they argue, imposes artificial restrictions that may not be necessary, depending on the context in which the devices are used. For example, a device that is used for first line screening with the aim of referral to another, more specialized, healthcare provider for further diagnosis and treatment, can have a lower specificity compared to a test that makes decisions 
about, or monitors, the treatment itself [5]. Therefore, seeing efficiency and reliability above all might be shortsighted. This is also indicated by other research; in a study that compared 12 different combinations of hepatitis $\mathrm{C}$ virus-diagnostics that were either PoC, lab-based or a combination. The cheapest strategy turned out to be a two-test combination system, first using a lower-specificity PoC antibody test followed by a confirmation via an RNA PoC test. All one-step strategies showed higher false-positive rates and were not cost-effective under base-case assumptions. However, two-step strategies are highly dependent on the loss-to-follow-up rate (LTFU) [50]. Gift et al., reported as early as 1999 on the "rapid test paradox" and the connection between LTFU and PoC tests with limited sensitivity that lead to better treatment outcomes [51]. Other authors also agree with this notion, and argue that a very affordable test and one with very good stability at high temperatures, but with suboptimal sensitivity, may still be extremely beneficial in tropical settings [26].

Another aspect, indicating that focus on raw diagnostic prowess might be overrated and the important part may be the accessibility to diagnostics in the first place, is found in the fact that only $28 \%$ of the inhabitants of Africa have access to advanced healthcare facilities. Tests that need only minimal infrastructure could give an additional $47 \%$ of the African population access to diagnostic tests. While improving the accuracy for bacterial pneumonia tests in advanced healthcare facilities only led to 119,000 more disability-adjusted life-years (DALYs) saved per year, 263,000 more DALYs could be saved annually if this test would be made available for rural sites with minimal resources [43]. This was also found in another study, using syphilis as an example. A PoC device, that would require minimal laboratory infrastructure, could prevent 138,000 
congenital syphilis cases and 148,000 stillbirth per year. A PoC device that would not require any laboratory infrastructure at all would prevent 201,000 cases and 215,000 stillbirths [22]. In the end, a perfectly assured device might be unrealistic, as several of the ASSURED criteria work against each other and especially against the affordability. The higher the accuracy the less affordable the device will be. Therefore, it is important to choose the right battles.

Another aspect criticized is that PoC tests often focus on a single disease; however, healthcare workers in LICs are concerned with syndromes of unknown etiology [20]. Additionally, having one test measure several factors would give the healthcare provider more diagnostic security, and save time.

\subsubsection{Steps towards an Effectively Usable PoC Device}

There are several possibilities that can help tackle those challenges. Multiplexing might be a large step forward, and nowadays several methods for multiplexing are possible and being developed $[4,22]$. This would reduce the relative costs for each test since they can use the same framework and are regulated at the same time. Healthcare workers are often interested in many different factors connected to one disease. Putting them together in one test can show hugely beneficial. Multiplexing also has logistical benefits, as there is less to track; associated equipment can be shared and several tests can be made while worktime is not increased.

Several authors suggest modern microfluidics to alleviate some of these problems. In sample preparation, for example, all the technology of DNA/RNA extraction and purification can be 
included in a small cartridge with very easy usability [43], reducing the need for external equipment and thus reducing cost [21]. The improvements in biosensor and microfluidic creation already helped make HTP devices possible due to the optimized microstructuring of transducers (nano-wires, nanopores, nano-particles), microspotting of the sensitive element and integration in low volume microfluidic channels [4]. Many other authors agree and see great potential in microfluidics for LIC-PoC [52-55]. The widespread use of smartphones is considered in many publications as a possible readout-device for optical platforms, as well as an ICT-connector [56-60].

To prepare nucleic assays for LICs environments, one major problem is assay stabilization, as PCR mixes require cold storage. Lyophillization would be an option to create assays that do not require a cold-chain. The problematic part is the diverse array of compounds used that are incompatible with freeze-drying, such as glycerol. There are mixes that are possible to freeze-dry; however, the amplification efficiency will likely suffer [43]. To improve ruggedness, functions should be reduced to the essential and have integrated quality control, and local production may help with access to support and consumables [61].

\subsection{The Market}

\subsubsection{Market Introduction}

The step from prototype towards market introduction seems to be the most taxing step, as low-cost PoC devices are being developed in many laboratories in HICs, but not being adopted as well in LICs [28].

\subsubsection{Funding and IP}


Funding is not only a consideration of research, but also of valorization. Even when funding for novel research is available, additional funding for economic aspects, such as manufacturing, distribution and maintenance are harder to find, resulting in the abandonment of projects by the original researcher due to cost, as well as deterring investment and interest from companies, which favor secure, established products [26]. Bringing new medical devices to the market is a costly enterprise. This is investigated more in depth in drug development where it is estimated that developing a new drug exceeds USD 1 billion in cost, takes more than 10 years and only $11.8-21 \%$ of new drugs are approved. There are no discrete numbers on this for medical devices, given that the field is highly diverse with an estimate of over 500,000 different types of devices, which span from X-Ray machines to hip implants with different diverse aspects that have to be taken into account for regulation, such as repair, maintenance and wear [62]. These processes can take years until a patient reaps the benefit of a new product, if it reaches the market after all [63]. This might make it unprofitable for a company to conduct fundamental research for LICs PoC if there is not an immediate benefit in reach [3].

Next to funding, intellectual property (IP) proves to be a large barrier for the development of systems upon existing technologies. Especially IP on molecules and genes make this increasingly difficult [4]. Existing patents covering biomarkers and even entire organisms exist, and there are large IP barriers in diagnostic platforms and/or components of diagnostic platforms [21,22]. One solution for IP considerations are new private-public partnerships, such as the World Intellectual Property Organization (WIPO) Re:search consortium. WIPO is the UN agency tasked with developing international IP systems 
fostering innovation for NTDs that benefits everyone. WIPO Re:search enables members royalty free use of infrastructure, compound libraries, IP assets and know-how. The WIPO consists of 107 members in 30 countries. IP Licenses can be used royalty free and new IPs generated are retained by recipient member, and not the member of the original IP asset. Often, WIPO Re:search members are from LICs. This creates benefits for everyone, and LICs contribute local know-how and have access to patient samples, while companies, often from HICs, contribute their research power and IP. The company benefits from heightened corporate social responsibility, access to other IP, business opportunities and access to other experts and know-how in the field; as well as networking possibilities for LICs, which are emerging markets $[3,64,65]$.

\subsubsection{Funding and Intellectual Property}

High regulatory barriers and strict healthcare standards are also a significant barrier to the introduction of innovations $[4,5,20]$. While good regulations are important, they are difficult and require substantial expertise to navigate them, which discourages innovation, especially in LICs where profitable returns are unsure. Clear and straightforward national policies for diagnostic evaluation and certification are key [20]. The pharmaceutical world is more advanced in setting up harmonization infrastructure, which is still lagging behind for diagnostics. In total, 23 countries in Africa banded together and pledged to harmonize the approval method for diagnostics, which is a huge step, as companies do not have to acquire approval for each country separately [66]. One other possible solution offers a WHO program that examines the quality and the safety of HIV and Malaria tests. This "prequalification" is 
a helpful guide for LICs, helping governments to speed up the approval process [26].

\subsubsection{Integrated Market Expertise}

Missing collaboration between academia and industry might be another bottleneck encountered when bringing inventions from the lab to the market. A multidisciplinary team for market introduction is, just as it is for research, an important part of the valorization trajectory [4,21]. A study of 358 medical devices for LICs found that only 134 met the study requirements to count as "commercialized" [61]. In addition, of the hundreds of devices beyond this particular study, many likely failed to commercialize because of, among other reasons, a failed transition from prototype to market introduction [61]. Studies have also indicated that good policy plans for quality systems and supply chains improves the accessibility if placed prior to the POCT program [20]. Those aspects of quality control and assurance and supply chain managements are what companies have strong expertise in. Those aspects are usually not considered enough in the prototyping phase, while scaling up is still not around the corner, this becomes a barrier later on into commercialization (vide infra). The expertise of companies can help to navigate the complexities of scaling and implementing a medical device and effective delivery mechanisms [21,61].

Therefore, it is not only necessary to have a research and valorization team, but also specialists for regulation, culture and policy [21]. Especially researchers in low-incomecountries often lack the knowledge spanning all involved fields, from discovery and research to market introduction [3]. The device must consider local and regional constrains, 
involved stakeholders and their needs and the capacity of the local healthcare workforce, but also social and cultural contexts for things that can easily be overlooked e.g., whether blood sampling is easily accepted [5,22,61]. One target group that should be especially focused on, but are often not included, are the end-users, who should be integrated in each step of the design process $[20,28,45]$. To this end, limited international (especially transcontinental) collaboration is problematic [21].

\subsubsection{Market Penetration}

\subsubsection{Device Quality}

After the introduction of the product, other aspects become important for wider market penetration. One problem is a bad batch-to-batch reproducibility especially in lateral flow devices that hamper upscaling [21]. Low device quality and low reproducibility can lead to decreased trust by healthcare providers and thus affect the adoption of other devices negatively [28]. This is one of the greatest concerns of rural LICs healthcare practitioners. In interviews, key-stakeholders expressed the need for diagnostic scale-up, but they also had concerns about reliability, as well as supply chain management and staff training [45]. This was confirmed by another study, reporting doubts from healthcare practitioners regarding test trustworthiness with either the accuracy, the robustness or the clarity of results. Adding to distrust is the concern of counterfeit tests being delivered [49]. Participants of the study found PoC critical for improving healthcare but judged their current form as not suitable for the local context. 
Point of Care Diagnostics in Resource-Limited Settings

\subsubsection{Economic and Social Placement}

Even a theoretical PoC device that achieved all ASSURED criteria perfectly might not be sustainable and gain market acceptance if they do not have a viable business model attached to them [45]. It is important to consider that this model fits in with the real-life workflow patterns. This means the PoC devices need to be integrated into the existing healthcare settings and real-life contexts of LICs to be successful.

The large importance of proper economic placement is shown by the fact that even inaccurate devices can be a market success if the stakeholders along the value chain profit from their use. For example, inaccurate serological tuberculosis tests, which the WHO advises against, are very common in private healthcare facilities in India, as well as in 17 of the top 22 other countries most affected by the disease. Private doctors earn referral money and other incentives for ordered tests, leading to an overreliance on inaccurate diagnostics due to economic incentives [5,67]. While quality is of course of the utmost importance, this example shows that, for market penetration, the importance of correct economic placement and integration into existing value systems is a key factor, which sadly can even outweigh actual clinical performance. Therefore, when aiming at maximum market penetration, the whole market with all economic influences, costs and workflow has to be understood. This is just as important as delivering a device with the best quality possible [45].

Other examples of misplacement of PoC testing in the market can be found in India and South Africa, where many wellworking and cheap (USD 1 per test) PoC tests are available for different diseases, such as HIV, Malaria, Dengue, Syphilis and Hepatitis. Yet many of those tests are still not commonly used 
at home, the physician's office or even in rural healthcare clinics arguably the target environment for PoC. Instead, testing happens foremost in laboratories and hospitals, whereas small independent laboratories are the major users of PoC devices [5]. Lab personnel are often skeptical against testing outside of controlled settings, as they lose control over testing and quality assurance and it interferes with their business model $[20,45]$. This shows how devices that are meant to be PoC can, due to mismatching interests in the established market, end up in a position where they cannot fulfill their intended purpose.

The placement of a new PoC device into an already existing healthcare system requires satisfying challenges and questions raised by every stakeholder. Who bares which part of the financial cost? What economic incentives are offered to various stakeholders? How is the training handled and how are information and communication technology used for reporting? Answering these questions in a satisfying manner, might be as crucial as the device itself $[5,45,68]$. Clear national guidelines for essential steps i.e., evaluation, certification, supply chain management, financing, training and expertise need to be put in place [20]. It also has to be pointed out that healthcare providers often feel that they do not have any influence on the decision regarding the availability of PoC devices, but instead "use what they are given" [49]. This shows that they are often not taken into account in this process. Healthcare practitioners expect decision makers to lay out the plan and define the use of PoC devices in the context of local epidemiology. Decision makers also need to take care of training before deployment and give adequate guidelines on how to proceed after a positive or negative results [49]. 
Market penetration is also heavily dependent on price. Here, the willingness to pay is an important aspect. A guideline to evaluate the affordability is $1-3 \times$ the GDP per capita per quality adjusted life years, which is gained through the intervention [50]. However, this calculation is not commonly shared as a good estimate and it may not lead to the best investment if it means loss of funding in other areas. Society might not be willing to contribute the necessary sums just because of cost effectiveness [69,70]. Another factor might be that patients themselves are not willing to pay the suggested price as they lack money and cannot invest in the long-term benefits. In Kenya, $51 \%$ of healthcare expenses are payed out of pocket, healthcare costs are often covered through "harambees" fundraising events in the community; $46 \%$ of the population only has USD 1 or less to spend each day [28]. HCTs platforms have high fixed costs for the PoC device itself and, therefore, the cost per test is strongly dependent on the use case and the workload of the device to be economically justifiable [71]; therefore, those diagnostic devices, with their high implementation cost, are considered as too expensive for widespread use [70]. A good example for this is HIV diagnosis and monitoring. Since viral counts are difficult to do in the field, often a laboratory procedure is required. While PoC HCTs devices are established on the market, widespread use is limited by the initial high cost of the device. However, research showed that it can be viable for clinics that have a moderate or large amount of patients to have such PoC HCTs devices for HIV detection, as the initial high cost of the device can be distributed over more patients. This is also true for the usage time. The longer the device can be maintained, the more costeffective it can be. With 50 patients per month, a reasonable assumption for a clinic in South Africa, the overall cost for antiretroviral therapy (ART) monitoring would be only USD 45 
higher (USD 210 compared to USD 166) than the laboratory procedure over a time span of 5 years. However, assuming only 10 patients per month, this would increase to a USD 183 additional cost over the same timeframe. Price is also dependent on which biomarker is analyzed, as viral load was, in the 50 patients per month case, just as cost effective as in the laboratory, while CD4+ count and creatinine test were more expensive [71]. The notion of cost effective PoC ART monitoring is supported by several mathematical simulations from South Africa, Zimbabwe and Mozambique, especially when all the costs are taken into account. A PoC test that enables better ART linkage can be tremendously more expensive per test, and still save follow-up costs in the long run, due to better immediate and consistent treatment, as well as greater reach [72-74].

While many simulations of cost effectiveness base their assumption on high-prevalence areas, others remark that especially for "the last mile", in areas with lack of infrastructure, $\mathrm{PoC}$ might be one of the only viable alternatives for the hardest to reach $10 \%$ of patients, as transport networks get more and more difficult to establish in remote areas [75]. Despite the low volume of patients making cost-effectiveness more difficult, it is estimated that an optimal placement of $\mathrm{PoC}$ viral load tests on-site and in PoC hubs still can reduce the price of a test by $6-35 \%$ by avoiding high transport cost in remote areas [76]. Finally, it has to be noted that, although PoC tests could significantly improve the healthcare system in LICs, their impact will depend on the specific disease or condition they are employed for. Therefore, the successful implementation of $\mathrm{PoC}$ will require a rigorous study of the overall cost-benefit ratio of any proposed $\mathrm{PoC}$ test, specifically addressing the disease it is meant to diagnose. 
Point of Care Diagnostics in Resource-Limited Settings

\subsubsection{Product Distribution}

Limited infrastructure in LICs not only results in low return-ofinvestments for companies, but also makes the distribution of the device and technical support more difficult, which might discourage companies or hamper the market penetration [26]. Stock outs and supply network problems are a massive obstacle to market penetration. Studies on PoC accessibility and supply chain management reported several stock outs of PoC devices. In a scale-up program for syphilis tests from an NGO-led pilot to a ministry of health operated large scale operation in Zambia, half of the pilot sites suffered at least one stock out. PoC for pregnant women also reported stock outs in several stages of the study, with up to $60 \%$ of sites reporting stock outs. The longest time the device was out of stock was a median of 6 weeks. In Uganda, malaria diagnostic tests were only available in $24 \%$ of 125 lower healthcare facilities, and $72 \%$ of community healthcare workers did not receive malaria testing kits for 6 month [2]. In antennal clinics in Guatemala, almost half of women could not be tested for HIV, Syphilis and Hepatitis B, in part because of stock outs [2,77]. Test kit stock outs are also reported from Uganda and Tanzania [78] and are a major concern to healthcare providers [49].

Here, the supply chain is a big point of failure, mostly due to irregular supply, poor forecasting, selection of diagnostics, insecure procurement systems, delayed distribution systems, poor quality assurance and inadequate stocks $[20,79]$. This has also been confirmed by healthcare workers who are concerned about the reliability of the supply chain [45]. Human resources are often not considered in the supply chain management, leading to bad planning and overstretched systems [66]. 
An innovative solution for the fast distribution of medical products in LICs is shown by the company Zipline, which uses remote drones to distribute blood preservations to hospitals in need all over Rwanda. Replacing the delivery from taking hours by Motorbike, to mere minutes via a Zipline drone, this innovation reduces the number of blood stocks the hospital needs and thus reduces waste due to expiry. In emergencies, matching blood can be delivered within minutes, something impossible with motorcycle rides that could take up to 5 hours $[80,81]$. In Zipline's system, the blood packs are dropped from the drone via a little parachute, hence a pickup of bloodsamples from remote areas is difficult, since the drone cannot independently land. However in the recent COVID-19 pandemic, Zipline collected COVID-19 test-samples by car and sent them bundled together to large hospitals via their drones, while also distributing to hospitals other COVID-19 related necessities $[82,83]$. Such innovations might help to counteract shortcomings in delivery-planning and infrastructure or deliver tests and consumables that have limited shelf life or limited temperature resistance. A pickup service for test samples in rural healthcare clinics, for further diagnostic procedures for example in a two-step process might be possible with new developments of drones that are capable of vertically taking off and landing, and are currently developed, for example by DHL [84].

\subsection{The Usage}

Limited testing capabilities are often a bottleneck for adequate therapy. This is especially observable in HIV treatment, where CD4+ counts and viral load are used to monitor antiretroviral therapy (ART). In Sub-Saharan Africa, the median of patients that were retained between HIV diagnosis and CD4+ count was 59\% [85]. HIV is a good example of a disease that is difficult 
to get a conclusive diagnosis on in the field as it needs a nucleic acid test that usually has to be performed in a laboratory with trained personnel $[86,87,88]$. While there are several HCTs PoC platforms available that can conduct HIV monitoring, such as the GeneXpert (Cepheid), the PIMA CD4+ (Abbot) or the Alere q (Abbot) [89,90,91], their high initial cost are still a problem. This makes ART therapy challenging to start and monitor in rural areas. Therefore, not only is supplementation with tests an important factor, but also the whole infrastructure of usage in combination with treatment, especially in rural healthcare settings where the different parts need to act together to create sensible plans for PoC testing and treatment delivery. Due to this interconnectedness of different factors surrounding the end-users, several bottlenecks can appear. HIV is, therefore, a good example of how healthcare, as well as patient management, are integral factors and can negate any positive effects that PoC can bring, if they are mishandled.

\subsubsection{Healthcare Management}

Political will towards PoC might be reduced when PoC tests lead to more demand for treatment, while at the same time treatment capabilities are scarce [5]. On the other hand, PoC diagnosis might not be feasible (for a test and treat scenario) when adequate treatment capabilities are not in place [70]. When treatment is available, patients might also just opt-out of tests in favor of direct use of medication, such as over-thecounter-antibiotics. This has been reported in Thailand, where missing information about disease origin among the public leads to a preference of medication instead of proper diagnosis, as medication is connected to symptoms instead of disease origin. For example, patients associate antibiotics with the symptoms of a bacterial infection instead of the infection itself and thus demand antibiotics even when the reason for the 
illness is not bacterial in nature but has similar symptoms [92]. PoC testing might offer proper diagnoses, preventing people from self-diagnoses and taking inadequate medicine. This also shows the importance of looking at PoC applications not independently but in the whole context of the healthcare system, that acts and is acted upon by various factors. Some researchers assess that the introduction of a PoC device into a system that is already in place changes the role from a technical to a social device. However, this also has its upsides a more elusive benefit of why healthcare workers in rural areas might want to use PoC testing is the psychological effect it can have on patients, giving healthcare workers more certainty which, in turn, transfers to the patient and improves compliance. Patients also might overestimate the capabilities of the tests, which encourages compliance [92]. PoC can provide evidence without the need of laboratory infrastructure and highly trained lab technicians [21].

\subsubsection{Patient Management}

In addition to the infrastructural bottlenecks related to the availability of electricity and water, which were discussed in the research section, one of the most immediate problems is patient management. In rural areas, traditional laboratory diagnostics are limited by distance to central laboratories. For laboratory diagnostics, samples, for example, in the form of dried blood spots, have to be transported via motorcycle, creating problems of long turnaround times for test results of up to two weeks and the danger of sample damage or loss. Another problem is the loss-to-follow-up when patients have to either wait for their results or have to go to another facility. This may be a problem for two-step diagnostic processes in which the second step is not PoC and the patient has to return or travel to a second facility [50]. Especially in rural LICs areas, there 
are large barriers to get to healthcare facilities, due to poor transport infrastructure and time constraints that might prevent a second visit [70].

For example, a third of the women in Ghana live further than two hours away from facilities with the capabilities of emergency obstetric and neonatal care [20,93]. Barriers such as these create gaps in the diagnostic and treatment pipeline and can lead to high levels of LTFU if patients do not return to collect their results and start treatment $[44,88]$. However, this also shows that highly effective laboratory diagnostics might not be suitable in LICs, even if their sensitivity and selectivity is far superior to PoC devices. If they pose the risk of LTFU, a one-step PoC device might be the more pragmatic and better solution [46]. In HIV diagnostic and treatment, the decentralization of diagnostics from large hospitals to rural healthcare centers (RHC) proved essential to give people outside of urban areas access to therapies, such as ART. Before the implementation of testing in RHCs, the rate of LTFU was unacceptably high [94] as over half of patients did not return to get their results [95].

The large potential of PoC diagnostics in this context is, in part, due to their ability to ensure the start of treatment in the same encounter, which is essential as the rapid initiation of treatment is immensely important in diseases such as aids and tuberculosis [45]. In India, just one round for combined screening and treatment of HPV reduced the cervical cancer rate and mortality for over 30-year-old women by 50\% [96]. For this reason, the WHO recommends a screen and treat strategy for 30-39 year old women [70]. A PoC test-time of under one hour from test to result would be ideal, as treatment can follow in the same encounter [22]. In a healthcare worker 
survey, the participants argued that a sample-to-answer time of less than an hour is indeed optimal [43]. This forms a barrier for most nucleic acid based tests, which take several hours [43].

Some researchers therefore argue for more holistic thinking in terms of health services. There needs to be better linkage that connects testing, diagnosis and treatment [66]. Given the large impact LTFU has, resources could also be used in preventing LTFU instead of perfecting diagnostic devices [70].

For example, getting infants on ART could be achieved with combinations of tests of different sensitivity, but proper linkage. The initiation rate of $71 \%$ could be achieved with PoC devices with a limited sensitivity of only $72 \%$, but a successful linkage rate of $99 \%$, or with a test of $100 \%$ sensitivity and $70 \%$ successful linkage $[86,88]$. Some researchers suggest that $\mathrm{PoC}$ tests should be evaluated just as much on their ability to facilitate linkage as they are on their performance [88]. As demonstrated before, the cost-benefit also favors a two-step system. The absence of functional referral systems is seen as a huge roadblock by other authors as well [95].

Therefore, information technology plays a key role in the context of PoC, maybe even more so than new and better devices themselves. The rapid reporting of results and counseling via mobile phones are essential for a decentralized use of PoC. Mobile phone-linked PoC devices can also assist in data capture and quality control, medication distribution, PoC tracking and data storage $[5,43,97]$. The usage of mobile phones in this way is generally categorized as mHealth, an expanding subfield of eHealth, and concerns itself with the use of wireless technology instead of connection through ordinary landline infrastructure, such as in eHealth. This is especially interesting for LMICs, as mobile phone usage outperforms 
other communication infrastructure usage [98]. In total, $70 \%$ of the 7.4 billion users of cellular phones reside in LMICs, and especially in sub-Saharan Africa, mHealth had a rapid expansion, making this approach hugely promising $[20,99,100]$. The response to Ebola PoC in the last epidemic might serve as an example, as it was very fast as a result of the effective surveillance systems in place. However, healthcare systems are slow to use this connectivity to their full potential [66]. This is starting to change as mHealth is more utilized. In a review of 255 studies of mHealth applications, 93 studies fell into the realm of health monitoring and surveillance, the second largest group with 88 publications concerned themselves with raising health awareness [98]; another study found the most used context to be increased patient follow-up, as well as patient compliance [100].

\subsubsection{Training}

While large hospitals in central areas might also have an appropriate workforce, the staff in rural healthcare clinics, which are the main access points to healthcare for the rural population, consist mainly of untrained individuals or inadequately small workforces; often just one doctor, nurse or pharmacist, with the possible addition of lay healthcare workers (LHWs) [43,61,95,101].

Human resources are in surprisingly short supply when it comes to healthcare workers and may stretch out the system, especially in rural sites [26,66]. Additional onsite testing could put even more strain on the already overworked staff; as already mentioned, this might be a major problem if there are no additional staff or incentives available, and might discourage PoC use $[20,43,102]$. There is also a problem in the lack of educated healthcare personnel, especially in Africa, 
which has over $24 \%$ of the worldwide disease burden while only having $2 \%$ of physicians of the world $[61,103,104,105]$. The shortage of skilled healthcare workers was seen as a problem by several authors $[20,49]$.

Surveyed healthcare workers assessed PoC diagnostics as easy to use; however, they still expressed fear of knowledge gaps among their users and concern of incorrect use. For example, the use of a wrong buffer solution or no buffer solution at all was observed [49]. Other researchers also reported reluctance for PoC in LICs due to the need for training, and the costs associated with implementation, as well as diagnosis [45]. It is especially feared that lay healthcare workers will not have the adequate training or knowledge to conduct even simple $\mathrm{PoC}$ tests, which could lead to inaccurate results that could damage the perception of PoC in these settings [20]. Despite those fears, the world health organization recommends task shifting to LHWs to meet human resources needs $[78,85]$ and it is a tool that is increasingly used to combat the estimate estimated shortage of 7.2 million healthcare workers, which is the most severe in Sub-Saharan Africa [106].

The question is, can task shifting from healthcare professionals to LHWs be achieved without a loss in reliability of test results. Lay health workers provide an important opportunity to give more people access to healthcare; this is especially the case for rural areas. However, medical devices are usually not designed to account for task shifting [61].

Some argue that task-shifting has to be accommodated when designing the PoC device. The interface has to be straightforward and user-friendly, even for laymen, which is often not considered in the design phase [21]. Ideally, the device should be a fully autonomous, robust, "black box" [4], 
which is fully automated with a simple interface and everything integrated into a simple "sample to answer" process [43]. Others suggest that the technological complexity must be as simple as a home pregnancy test [22], or assess modern PoC diagnostic platforms used for CD4+ testing as too sophisticated for usage in LICs [44]. Laboratory professionals also doubt that diagnostics can be performed by lay-healthcare-workers with appropriate quality assurance $[5,45]$. This viewpoint might be understandable, given the findings of doctors observing the wrong use of lateral flow devices [49]. The question, therefore, remains whether PoC devices demand usage by healthcare professionals.

Research is suggesting otherwise. A study about task shifting for the use of the HCTs Pima CD4+ Analyzer (Allere) in Namibia showed that lay-health-workers can produc valid tests as nurses. In a large study of 1429 CD4+ tests, in which 500 were performed by nurses and 929 by LHWs, the reception of test results by the patients was in favor of LHWs, with $98.1 \%$ contrary to $95.6 \%$. LHCs were only slightly slower, with a median turnaround time of 21 minutes compared to 20 minutes for nurses. However, both were a tremendous improvement from the turnaround time of a laboratory test, which had a median of 4 days (IRq 2-8). Therefore, task shifting to LHWs may be an appropriate choice, even for more complex tests [85]. Other studies agree that LHWs can perform rapid testing just as well as trained laboratory staff, if trained properly. However, when implementing a training program, it has to be considered that the training package is adapted to the local environment [78]. LHWs in Malawi have named a lack of disease- and job-specific training as a key problem hindering their role as TB care providers [106,107]. Lateral flow tests for HIV testing were so successful in their ease of use, that task 
shifting could be greatly implemented and the tests in LMIC are now often done by expert-patients or trained lay healthcare workers [95].

\subsubsection{Use by Trained Doctors}

In cases where trained medical professionals are available, the bottlenecks present themselves differently. One issue is time constraints. In India, doctors prefer clinical diagnoses coupled with empiric treatments over a higher diagnostic security. Broadband antibiotic prescription after only a short symptomatic observation is a common example; this is faster than doing an additional PoC test that might not even be necessary, just in order to make the diagnosis more secure [5]. The general overburden of doctors in India is one factor for this. Generally, visits only last a few minutes, which is generally not enough time for PoC testing. On the doctor's side, it is better for his reputation to treat several other waiting patients in this time and keep waiting-lines short. Many doctors only have a single room with one nurse as an assistant, even simple lateral flow tests are difficult to conduct under such conditions [5].

Awareness is another issue, which is suggested by the literature. Healthcare providers might not be aware of PoC tests on the market [5]. Lack of knowledge about PoC testing among people living in rural areas could be addressed by, for example, advertising and explaining the use of $\mathrm{PoC}$ tests for rapid diagnosis of specific diseases. This could raise awareness and prevent last-minute visits to the doctor. However, general awareness of PoC devices in Kenya was surveyed and is high throughout high-, mid- and low-tier healthcare providers and seems to not be a critical barrier. In total, $95 \%$ of healthcare 
providers in his survey could name a disease that can be diagnosed with PoC tests ( $71 \%$ could name two and $24 \%$ could name three); only $5 \%$ were not able to name any disease which has $\mathrm{PoC}$ tests available. However, only $10 \%$ of healthcare workers which named more than three PoC devices had actually applied them in their practice [28]. This indicates that the bottleneck is systemic rather than knowledge-based. Higher knowledge and usage about HIV tests were shown by doctors in richer hospitals compared to rural doctors. For example, in malaria diagnostics there is a wide gap between the knowledge about (57\%) and actual use of PoC diagnostics (36\%), independent of socioeconomic factors. It is suggested that this is because $\mathrm{PoC}$ devices for malaria are seen as of limited usefulness as the disease has strong symptoms and the prevalence is foreseeable due to the seasons. Another possible reason might be the lack of availability, the devices not complementing other diagnostic methods or greater success with other diagnostic methods, such as a symptomatic approach. For other identified diagnostics, the knowledge about the device was 1- to 3-times higher than the actual use [28]. Healthcare workers in other studies could identify various PoC tests. The most known ones were for Malaria, HIV, Syphilis, Blood Glucose and Pregnancy. These findings concurred with other surveys $[49,108]$.

\subsubsection{View on PoC}

With regard to the view of patients on $\mathrm{PoC}$, it was found that, according to healthcare personnel, patients were satisfied with PoC results (97\%) and would recommend them (96\%). However, only half of clinicians thought they would give reliably accurate results; $46 \%$ were unsure and $4 \%$ considered 
them not accurate. In total, $65 \%$ of healthcare workers used medication even on a negative test, showing that trust in the test is limited. This concurs with only $20 \%$ of healthcare workers stating that they rely on the test alone. The majority sees the test as a complement to other means of diagnosis, such as symptoms [28], as 54\% of surveyed encountered barriers preventing PoC use. The likelihood of encountering barriers correlated with hospital tier $(45 \%$ in high-end hospitals and $53 \%$ in mid-tier). In total, $50 \%$ of personnel who encountered barriers named reliability issues as a large problem; the second largest obstacle named was availability, with $46 \%$. Only a smaller percentage saw cost $(14 \%)$ and awareness or training deficiencies (12\%) as major obstacles. When asked about improvements to increase $\mathrm{PoC}$ use, the respondents replied with improved tests (44\%), improved reliability (22\%) and standardization (20\%) which was specifically mentioned, even though it was not in the survey as an answer. Oddly, increased availability was named by just $22 \%$, despite it being the second highest identified barrier. In total, $85 \%$ agreed that $\mathrm{PoC}$ is an opportunity for more affordable healthcare in Kenya [28].

From the first start of research in the beginning, to the view of end-users at the patient-side, the bottlenecks of PoC diagnostics along the value chain seem to be as diverse and as different from each other as the actors and circumstances that present themselves on the way, as is summarized in Figure 25. However, as Figure 26 shows, there are many possible solutions at each step as well, which are, next to technological advancements, often based upon the connection of different stakeholders. 
Point of Care Diagnostics in Resource-Limited Settings

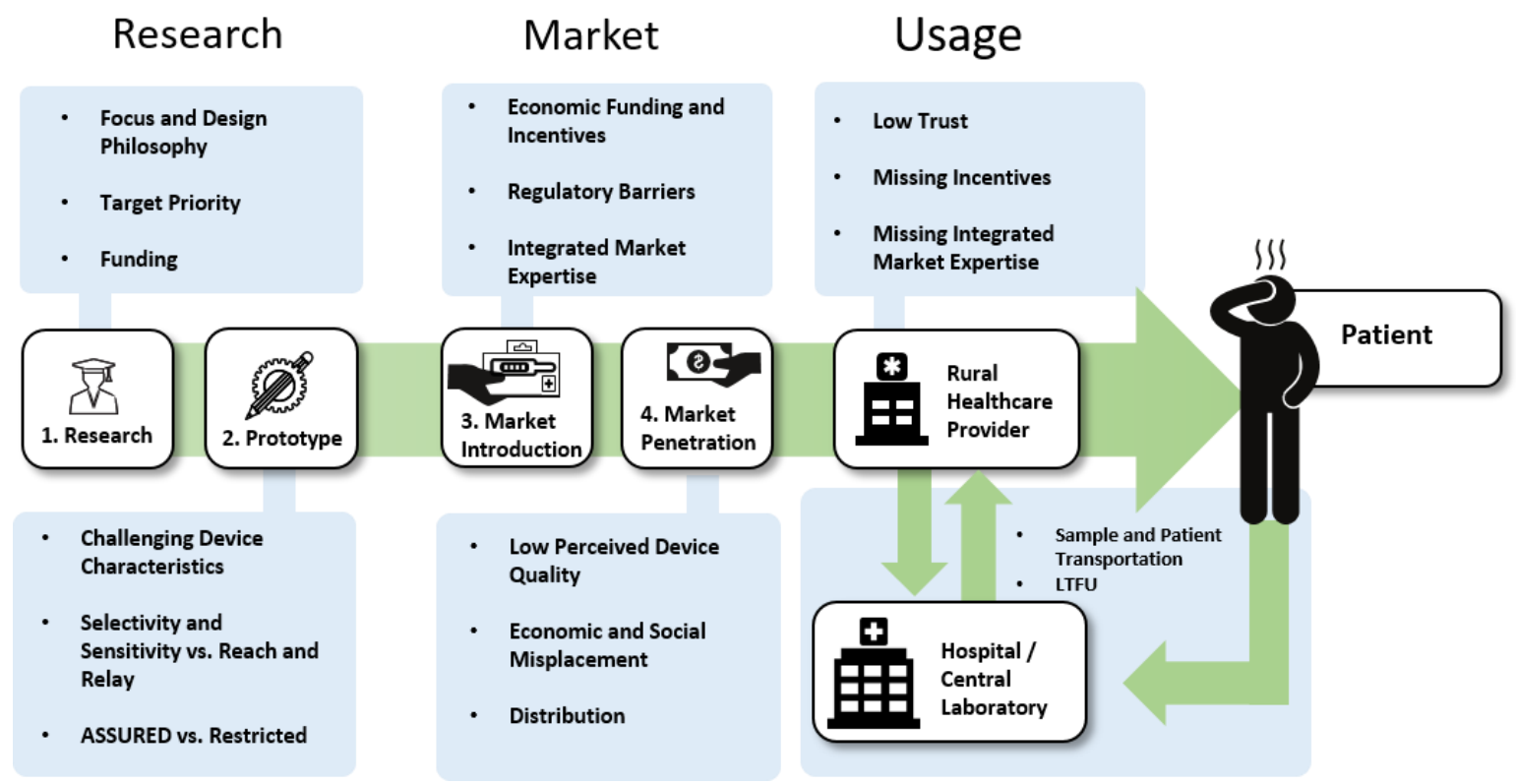

Figure 25 - Identified Problems along the value chain. 


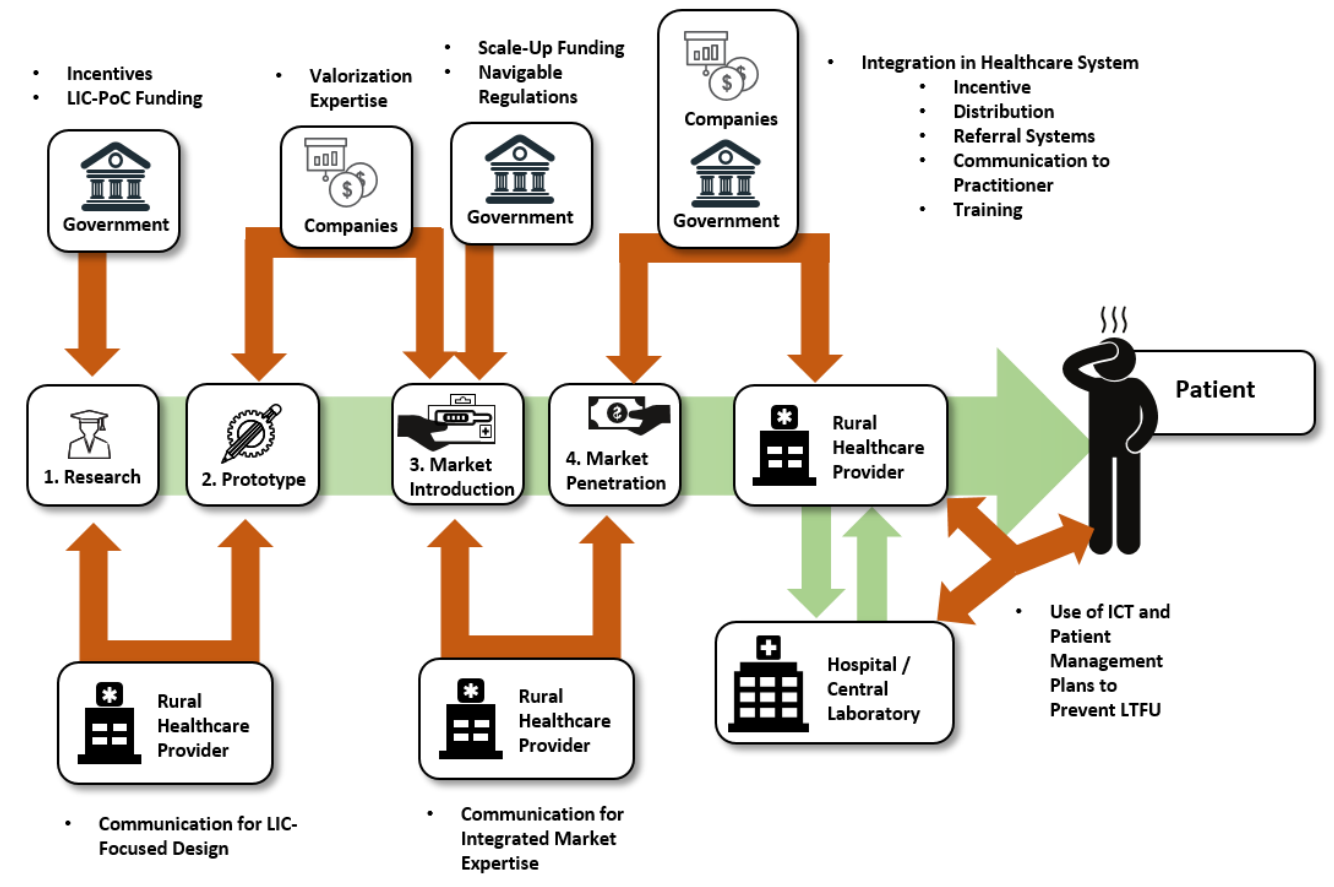

Figure 26 - Identified Pivotal Points and Influencing Forces. 


\subsection{Reflection}

\subsubsection{Main Findings}

Fundamental research always starts with funding, and it is, therefore, an obvious consideration. However, funding is not only needed here. Additional funding, as well as incentives for valorization is something direly needed to actually make the jump from a research principle to a medical device. Push- and pull-incentives are used with considerable success in drug development and might prove valuable if systems directed at $\mathrm{PoC}$ are in place. Connecting all stakeholders, such as research groups, companies, healthcare professionals, as well as governments and NGOs, is essential and enables IP considerations and licenses to be negotiated to everyone's benefit. Healthcare professionals' needs can be shared and taken into account, and company expertise in scale-up and distribution can be applied.

For the development of the device, one can ask the question of how important it is to be ASSURED. While many argue for sensitivity, specificity and reliability as a main point, there were other voices arguing for a more integrated view. For example, it can be argued that the needed criteria solely depend on the use-case. This notion has been supported by original research, showing that a two-step system can be cheaper, as well as more specific, despite the first stage not having optimal characteristics. However, for this to work, a proper integration into the healthcare system, with reasonable referral structures and minimal LTFU, has to be achieved. This might be realized by improved ICT structures and the use of mobile phones for diagnostic readouts. Tests can have shortcomings, if proper linkage to further tests and treatment are in place and LTFU is 
at a minimum. However, practitioners need to be aware of the test shortcomings to have a safe basis for decision making. The perceived low device quality can, and does, hinder effective PoC usage due to mistrust by doctors. Clearer communication of what $\mathrm{PoC}$ can and cannot achieve might give healthcare providers more certainty in their decision-making and empower them to trust the device and correctly interpret the received results. Clear guidelines for healthcare workers on how to use the results of a PoC device in the grand scheme of things need to be in place, including what the next steps for patients are, either for treatment or further diagnosis. These guidelines need to be beneficial to the healthcare worker as well, instead of just adding additional work. At this moment, it is more in the interest of a doctor in India with 30 waiting patients to simply give the patient antibiotics and send him/her home after a brief symptomatic assessment than to perform a PoC diagnosis, which takes more time. If the patient does not come back, the doctor may assume him cured and treat it as a success. However, this is not in the benefit of society nor the patient. Therefore, test and referral systems must be integrated in a way to lift the burden from the practitioner instead of adding them.

On the market side, the tremendous influence of a proper placement into the healthcare system and its incentive structure is shown by the use of subpar-PoC devices in India and other countries. If malfunctioning PoC devices can achieve widespread use, surely working diagnostics can be used if they are properly integrated into an incentive structure. The importance of incentive structure is also shown by PoC use in diagnostic laboratories in India and South-Africa, where PoC devices are misguided as cheap laboratory alternatives, instead 
of their intended goal as a fast, patient-side, diagnostic instrument.

A social problem that was identified as a large bottleneck is the lack of available workforce for testing. While the opinion of researchers regarding task shifting varies from skeptical to enthusiastic, there are interesting insights into its feasibility, arguing that lay-healthcare-workers can even conduct tests on more difficult platforms if trained properly.

\subsubsection{Outlook}

As research on PoC diagnostics continues, we will get closer and closer to versatile, accurate and low-cost detection methods that are more in line with the desirable ASSURED criteria. However, for the immediate success of PoC and for the benefit of patients in LICs, the research part might already be more advanced, compared the other aspects of the value chain. This is shown by the fact that already established PoC tests and resources are not nearly used to their full potential. A wide range of reasons can be identified, from missing funding for scale-up and lack of corporate incentives, as well as delivery problems and stock outs, to the problem of integration in healthcare systems and lacking trust by doctors. The lack of valorization of PoC in LICs seems to be a social and an economical problem, more than a problem of research.

Researchers will make progress for continuously improved devices, which will be easier to implement. However, the burden of implementation should not be solely put on the shoulders of scientists to discover novel advanced technical solutions for a perfectly ASSURED system. Tests that might not be perfect, but that are instead perfectly adequate for use are in reach, but missing incentive structures and lacking political attention will prevent their effective use. 
While Zipline is a company concerned with infrastructure and not with diagnostics, it still shows, in an impressive manner, how new technology can innovate a whole market, if there is political will and no old stakeholders that benefit from the status quo. In the case of Zipline, there was no real alternative for emergency blood delivery in an appropriate amount of time. The stage was free for Zipline's new and improved technology. In diagnostics, old infra- and incentive-structures from centralized laboratories to skeptical or time-limited doctors have to be overcome. While developments in research and innovation in $\mathrm{PoC}$ diagnostics over recent years are even more impressive than Ziplines drone systems, they also have to conquer larger infrastructural barriers. Zipline's CEO, Keller Rinaudo, stated that the technology is the easy part. It is more difficult to improve regulatory issues, acquiring and training the necessary workforce locally and creating awareness of their services to doctors and healthcare workers [80]. Mabey et al. provided us with another success story that further demonstrates that $\mathrm{PoC}$ tests need to address the whole value chain in order to be successful [109]. They implemented PoC tests for syphilis diagnosis and were successful because they did not only addressed a need in the healthcare system and offered solutions that adhered to the ASSURED criteria, but also managed to impact health care workers training, ensured that effective treatment was available and improved the local medical supply chain. These requirements are further illustrated by the case of blood glucose testing, the most striking example of a PoC success story in HIC. Blood glucose meters and test strips are often unavailable in LICs and even when present, other factors hamper their implementation, such as poor diabetes education and economical constraint regulatory issues [110]. Therefore, sadly, one of the most 
impactful PoC tools in HICs has not yet been able to achieve the same impact in rural, low-income settings.

Faced with the healthcare challenges in LICs, a transformation to a smart healthcare system, with real-time information flow and referral structures, will be necessary to make the most out of the innovations that come out of the lab.

Thus, there is a case to be made that scientific progress and innovation may not be the limiting factor, and other limiting steps seem to be hindering valorization at least as much. Those factors might improve, together with the economic development of LICs, but for now researchers need to take these social factors just as much into account as the technical aspects of new devices. The research and implementation of a new device have to be designed in synergy with its target location, instead of merely adapting to it later on. Advances for fast throughput devices of stable and long lasting reagents with frugal design and the integration of mHealth capabilities, and devices that have task-shifting in mind, will be of great help in this challenge as long as those technical advancements get connected to the local realities. mHealth connection will only be as potent as the referral structures that are in place to accept it. A task-shifting enabled device is only be as beneficial as the availability of LHWs. Therefore rollout, training and supply plans have to be integrated in and developed together with the device and in close connection to the target environment, which might be, at least in part, an aspect of a researchers work too. Research needs to break down as many barriers and enable the connection of as many stakeholders as possible in order for policy makers and companies to take the leap and bring $\mathrm{PoC}$ diagnostics to the patients. 


\subsection{Transition}

As stipulated at the beginning of the chapter, PoC devices need to have success on- and be distributed in the market in order to bring benefit to people in need for healthcare. And as postulated at the end of the chapter, we as scientists and engineers need to break down as many barriers as possible to improve the chances of market success. Here we also have to think actively about the bottlenecks our device will face in the greater context of its usage outside of our laboratory setting. With the structured information resulting from this review, the critical hurdles and bottlenecks for PoC devices are now brought to light and certain important aspects can be focused on in order to facilitate the success of a PoC device outside of a laboratory environment. Armed with this knowledge we can now continue to the experimental part of the thesis, where we first focus on the HTM as an affordable readout device and prove its applicability in UTI diagnosis. After this, we will focus on 3D printing of microfluidic parts, which, due to its affordability, can enable scientist in LIC to conduct microfluidic research right in the usage-environment. However, this is not the only advantage 3D printing can bring to LIC, in the future it can potentially alleviate many problems with stock management and enable on-demand production of parts for PoC devices. Therefore, it has the potential to be an enabling technology for biosensing in LIC and will be the second focus of the experimental part. 
Point of Care Diagnostics in Resource-Limited Settings 


\section{Biomimetic Bacterial Identification Platform}

Based on Thermal Transport Analysis Through Surface Imprinted Polymers: From Proof of Principle to Proof of Application

Adapted from:

B. Heidt, R. Rogosic, J. W. Lowdon, M. Desmond-Kennedy, K. Jurgaityte, J. Ferrer Orri, Y. Kronshorst, S. Mendez, E. Polyakova, H. T. Rice, F. Crijns, H. Diliën, E. Steen Redeker, K. Eersels, B. van Grinsven, T. J. Cleij, Phys. Status Solidi A. 2019 216, 1800688, DOI: 10.1002/pssa.201800688. 


\subsection{Chapter Context}

This chapter covers a proof of application for the diagnosis of urinary tract infections (UTI) using the heat-transfer method (HTM). Here, urine samples spiked with E.coli were analyzed using HTM, demonstrating its actual utility for medical diagnostics. While previous research already indicated the applicability of HTM for UTI diagnosis, these preliminary results were based on a single individual. In this chapter, we expand the study to a small group of individuals of different genders, to account for interpersonal differences such as a difference in $\mathrm{pH}$ and osmolality. Furthermore, it demonstrates that time sensitive measurements, close to the time of sample taking, are essential for proper diagnostic results as older samples show to be difficult to use for diagnosis. This connects with the findings of the previous chapter, which brought attention to the benefit of fast PoC measurements, especially in $\mathrm{LiC}$ where sample transport by motorbike to laboratories for conventional diagnostics can take a long time and cold-storage cannot be guaranteed and sample integrity can thus easily be compromised. Therefore, it is demonstrated that PoC able technology, like the HTM, can be a valuable solution to conduct patient-side diagnostics and assure sample integrity even in difficult circumstances.

\subsection{Introduction}

In healthcare, the subject of bacterial infection is pervasive and difficult to overlook. Accurate detection of bacteria is essential for correct disease diagnosis, treatment of infection, and the identification of potential outbreaks within the public. Additionally, the rise of antimicrobial resistance worsens the impact of bacterial infection and increases the necessity for effective detection of bacteria[1,2]. In the United States alone, 
at least [2] million incidences of infection of antibiotic-resistant bacteria are reported annually [3]. Moreover, the risk of acquisition of bacterial infection by patients already admitted to hospitals is an area of concern. These cases, known as Healthcare-Acquired Infections or HAIs, affect up to $10 \%$ of hospitalized patients and remains a problem in healthcare facilities worldwide.[4] The most common type of HAIs found globally are urinary tract infections (UTIs), which affect more than 8 million people a year in the United States alone $[5,6]$. Escherichia coli (E. coli) is the most common bacterium responsible for UTIs, accounting for $80-90 \%$ of all infections [7]. There is no minimum level of bacteria standardized by laboratories nor scientific literature for the diagnosis of UTI, but most laboratories regard a concentration of at least $10^{5}$ BACTERIA $\mathrm{mL}^{-1}$ in a patient's urine sample as the cut-off value for UTI diagnosis [8-10].

Conventional bacterial identification methods involve examination of cultured samples in the laboratory using timeconsuming techniques, with limited sensitivity and selectivity [11]. More advanced methods that depend on genetic screening, including polymerase chain reactions (PCR), are also used for bacterial identification [12]. Despite being fast, very accurate and effective at detecting bacteria, these methods require considerate sample preparation, the use of costly equipment and handling by trained professionals $[13,14]$. The current state of bacterial detection technology, in conjunction with the growing issue of bacterial infection worldwide, therefore calls for a detection method that is cost-efficient, easy to use and effective. Ideally, such detection methods would achieve point-of-care standards in speed and selectivity, being able to provide relevant results, expedite treatment, and limit spread of infection. Biosensors are a potential solution to the 
problem, as they are capable of providing accurate and efficient detection. They depend on both a bioreceptor, which recognizes a target analyte, and a transducer, which produces a measurable signal used to infer conclusions from the analysis [15]. In the case of bacterial detection, many existing biosensors have been based on antibodies or enzymatic receptors [16]. Despite their selectivity, they are difficult and costly to produce and suffer from limited thermal, physical, and chemical stability.

Synthetic receptors that mimic the sensitivity of these biological receptors can be made via imprinting technology and could offer a solution to this problem due to their cost-effective synthesis process and superior stability [17]. In terms of detecting macromolecular entities such as bacteria, surfaceimprinted polymers (SIPs) have shown considerable promise as effective biomimetic receptors. The microcontact imprinting technique, in which templates are stamped into a pre-cured polymer layer, has proven to be a particularly elegant method for creating highly stable, selective SIP layers [18]. After crosslinking of the polymer layer upon stamping and removal of the templates, microcavities are created on the surface of the receptor layers that display a high degree of morphological and functional compatibility to the template organisms, allowing them so selectively rebind to the layer. In this way, SIPs have been demonstrated to be excellent synthetic receptors for application in biomimetic sensor platforms for the detection of microorganisms such as yeasts or bacteria [19].

In recent years, the authors of this paper have successfully combined these SIPs with a novel thermal readout method for the detection of various targets ranging from low-molecular weight compounds such as neurotransmitters to large 
macromolecular entities such as whole cells [20, 21]. More recently, the method was extended towards the detection of bacteria in buffer fluids. The working mechanism of the sensor, the heat-transfer method (HTM), is illustrated in Figure 27; the SIP-coated aluminum chip is attached onto a copper block. The sample is heated from the bottom by an adjustable heat source and the temperature underneath the sample, $\mathrm{T}_{1}$, is kept constant by means of a thermocouple and a temperature-control unit that create a feedback loop. The transmitted temperature, $\mathrm{T}_{2}$, is monitored in time by a second thermocouple placed in a liquid measuring chamber. In this way, temperature transport can be monitored in time. Binding of bacteria results in a concentration-dependent increase of the thermal resistance of the solid-liquid interface which could be registered as a decrease in the transmitted temperature [1,22]. In this way, the current platform allows differentiating between at least eight different bacterial species and is able to detect bacteria in urine in concentrations that are clinically relevant for urinary tract infection (UTI) diagnosis [1, 23]. 


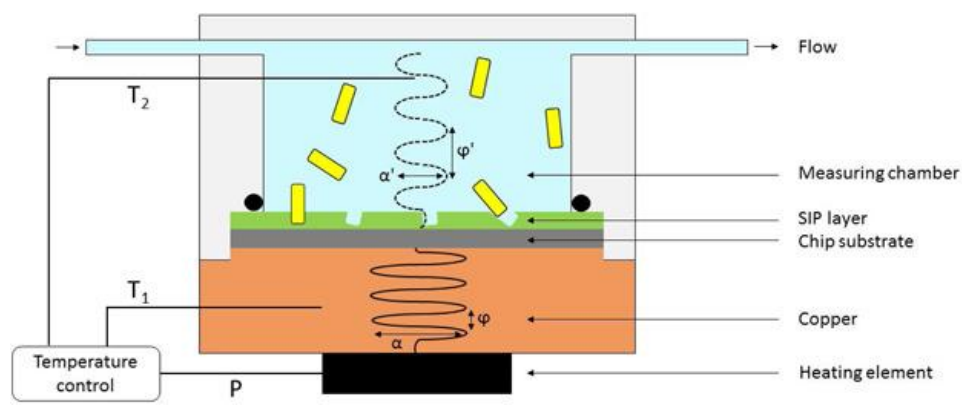

Figure 27 - Schematic of the thermal readout unit. The temperature of the heat provider (T1) is fully controlled by a thermocouple, a PID controller, and a heating element. A constant thermal current, is sent through the conducting chips and the SIP layer, resulting in a transmitted temperature profile registered by a second thermocouple. Reproduced with permission[23].Copyright 2017, American Chemical Society.

In this study, further investigation of the diagnostic capabilities of the thermal detection method for UTI detection is demonstrated, analyzing a broader range of samples. Urine samples from four subjects were spiked with varying concentrations of E. coli. Time-dependent temperature measurements using SIP-coated aluminum chips were used to construct dose-response curves and examine the effect of variables such as gender, age, and osmolality on the performance of the sensor. In addition, a sample was handled and stored for $24 \mathrm{~h}$ in order to examine the effect of transport and delayed analysis of the urine samples on the bacterial load present in these samples.

\subsection{Experimental Section}

Bacterial Culturing and Sample Preparation: The described strain of E. coli (ATCC 8739) were obtained from DSM-Z. Lysogeny broth (LB) was prepared according to the standard 
protocol and used as a source of nutrients to ensure bacterial growth. Twenty milliliters of LB was inoculated with a single colony of E. coli. The culture was left to grow, overnight at $37^{\circ} \mathrm{C}$ while gently shaking the flask at $120 \mathrm{rpm}$. Following this, $0.5 \mathrm{~mL}$ of the culture was diluted in a further $20 \mathrm{~mL} \mathrm{LB}$ and allowed to grow for $2.5 \mathrm{~h}$. The OD600 was measured to determine the initial concentration, centrifuged for $60 \mathrm{~s}$ at $6000 \mathrm{rpm}$ and diluted with PBS to obtain an imprinting solution of $1 \times 10^{5}$ bacteria $\mathrm{mL}^{-1}$.

Urine samples were taken from four of the co-authors, two males and two females volunteers with ages ranging from 20 to 35. These urine samples were sterilized by autoclavation to ensure they were not contaminated with any micro-organisms despite the fact that the volunteers did not suffer from UTI. These clean urine samples were used to create a stock solution of $1 \times 10^{5}$ bacteria $\mathrm{mL}^{-1}$ by diluting bacteria in a similar manner to that described above for PBS. From this stock solution, samples of $2 \times 10^{4}$ bacteria $\mathrm{mL}-1,3 \times 10^{4}$ bacteria $\mathrm{mL}^{-1}$, and $5 \times 10^{4}$ bacteria $\mathrm{mL}^{-1}$ were prepared.

Preparation of Bacteria-Imprinted Polyurethane Layers: Polyurethane layers were synthesized by dissolving $122 \mathrm{mg}$ of 4,4-diisocyanatodiphenylmethane, $222 \mathrm{mg}$ of bisphenol $\mathrm{A}$, and $25 \mathrm{mg}$ of phloroglucinol in $500 \mu \mathrm{L}$ of anhydrous tetrahydrofuran (THF). All reagents had a purity of at least 99.9\% and were used as received (Sigma-Aldrich N.V., Diegem, Belgium). The mixture was placed on the heating plate at $65^{\circ} \mathrm{C}$ for about $3 \mathrm{~h}$, while gently stirring until gel form. The prepared solution was diluted in anhydrous THF in a 1:5 ratio. Polyurethane layers were made by spin-coating the solution on aluminum chips with mirror finish at $2000 \mathrm{rpm}$ for $60 \mathrm{~s}$ each (Laurell model WS-650 Spin Coater). 
In parallel, PDMS stamps (Sylgard 184 silicone elastomer kit, Mavom N.V., Schelle, Belgium) were coated with bacterial solution in PBS and allowed to rest for $20 \mathrm{~min}$ in order for bacteria to precipitate. Excess solution was removed by spincoating at $3000 \mathrm{rpm}$ for $60 \mathrm{~s}$, to establish a bacterial monolayer on the surface of the stamp. The stamps were placed on the polyurethane layers and pressed gently. The polymer was cured at $65{ }^{\circ} \mathrm{C}$ overnight. After, the stamp was removed and $70 \%$ ethanol and PBS were used to wash off original bacteria, leaving cavities on the surface.

Sensor Setup and Measuring Methodology: The heat-transfer measurement was used as setup for biosensing assays. The SIP was pressed onto a copper block in the chamber of the biosensor, through which the heat wave passes. The temperature of the heating element $\mathrm{T}_{1}$ was monitored with a proportional-integral-derivative controller $(\mathrm{P}=8, \mathrm{I}=1, \mathrm{D}=0)$. The flow cell used had an inner volume of $110 \mu \mathrm{L}$ and was sealed by an O-ring which defined a surface area of $28 \mathrm{~mm}^{2}$ as a solid-liquid interface. A second thermocouple was used to measure the temperature inside the flow cell. More technical details on the setup can be found in ref. 20 .

First, the sensor was flushed with a sample of clean urine and the system was allowed to stabilize for $60 \mathrm{~min}$. After, the samples were flushed through such that the concentration within the sensor increased at the following intervals: $2 \times 10^{4}$, $5 \times 10^{4}, 8 \times 10^{4}, 13 \times 10^{4}$, and $18 \times 10^{4}$ bacteria $\mathrm{mL}^{-1}$. For each flush, $3 \mathrm{~mL}$ of bacteria-spiked urine was injected at a flow rate of $0.25 \mathrm{~mL} \mathrm{~min}-1$, after which the signal was left to stabilize for $30 \mathrm{~min}$. From the response, a dose-response curve was obtained. 
Imprint Characterization: Microscope slides were spin coated and imprinted as described previously. Microscopic imaging of the cell-imprinted slides was performed with an Olympus BX51 optical microscope (Biobased Materials, Chemelot Campus, Heerlen, the Netherlands) at 20X magnification. The open software ImageJ (National institute of Health, Bethesda, MA, USA) was used to determine the number of imprints. The average surface coverage was calculated from different spots on each sample, by taking the largest and smallest imprint sizes on the image.

\subsection{Results}

3.4.1 Surface Characterization and Calculation Surface Coverage

Optical characterization and analysis of a cured and an uncured SIP sample imprinted with E. coli were used to assess the quality of the imprints prior to measurement. Prior to this, a study was done to confirm that the coverage on the stamps used to make different SIP samples was comparable (see Supplementary Information). A heterogeneous distribution of the imprints throughout the sample was observed (Figure 28), with diameters ranging from 1.0 to $2.0 \mu \mathrm{m}$, as expected by the dimensions of the template E. coli reported as ranging from 0.7 to $1.4 \mu \mathrm{m}$ diameter [24]. Larger imprints may be attributed to overlap of multiple targets during the imprinting and curing process. Two different batches of the same target SIPs were analyzed, to calculate surface coverage. The first SIP sample, which was not cured overnight (Figure 28a), showed a low surface coverage of $9.16 \pm 1.41 \%$, corresponding to an approximated numerical coverage of $\approx 2.60 \times 10^{6}$ imprints per $\mathrm{cm}^{2}$. However, the analysis of a cured SIP sample (Figure 28b) 
showed a heterogeneous higher surface coverage of $12.40 \pm 4.64 \%$, corresponding to a two-fold higher numerical coverage of $\approx 5.05 \times 10^{6}$ imprints per $\mathrm{cm}^{2}$. These data are in accordance with a previously performed optical analysis on the same SIP preparation [22].
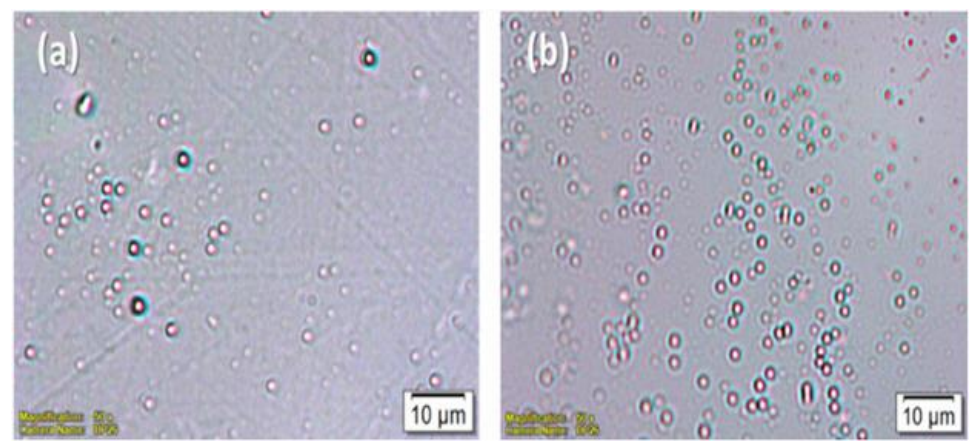

Figure 28 - Microscopic analysis of two E. coli SIPs at 50× magnification. a) Uncured and b) fully cross-linked SIP layer.

\subsubsection{Effect of Gender on Quantitative Detection of Bacteria in Urine}

The reproducibility of the previously demonstrated selectivity of the SIPs and sensitivity of the transducing principle in terms of bacterial detection in urine was studied. Therefore, E. coli SIPs were exposed to spiked urine samples donated by both a male and a female donor. The concentration was gradually increased in five steps of $2,5,8,13$, and $18 \times 10^{4}$ bacteria $\mathrm{mL}^{-1}$ by injecting $3 \mathrm{~mL}$ of a $2 \times 10^{4}$ bacteria $\mathrm{mL}^{-1}$ concentration initially, followed by two consecutive injections of sample with a concentration of $3 \times 10^{4}$ bacteria $\mathrm{mL}^{-1}$ and finally two consecutive injections of $5 \times 10^{4}$ bacteria $\mathrm{mL}^{-1}$. The 
concentration was gradually increased by stepwise addition of increasing concentrations, and the stabilization period was repeated until reaching the highest concentration (Figure 29). The time-dependent temperature data (Figure shows similar trends between spiked urine samples obtained from a male and a female individual as a concentration-dependent drop in $\mathrm{T}_{2}$ can be observed in both samples. 
Biomimetic Bacterial Identification Platform
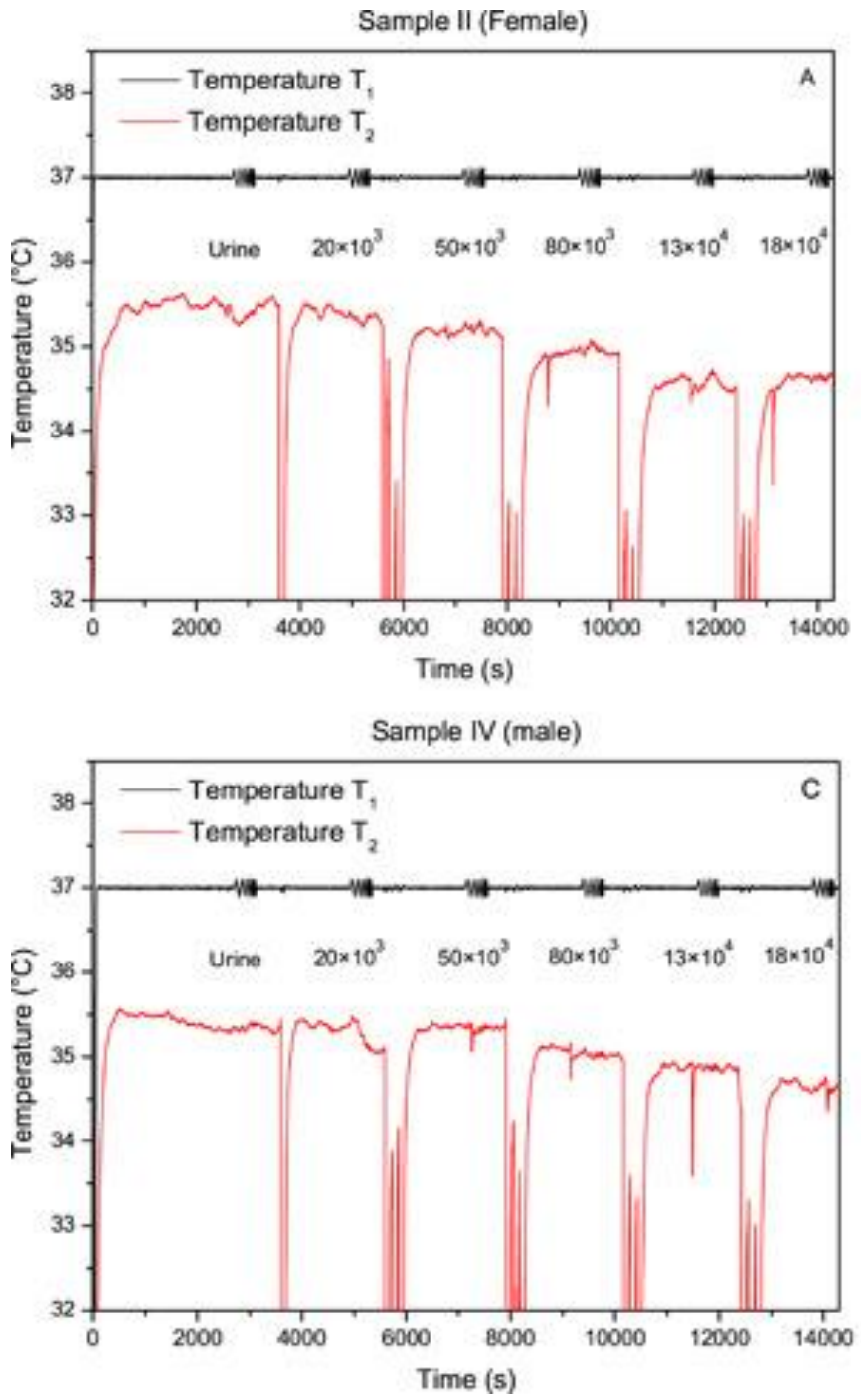

Figure 29 (a) and (c) - Time-dependent temperature response of an E. coli SIP in response to an increasing concentration of target bacteria. Picture showing the concentration dependent temperature decrease for both samples. 

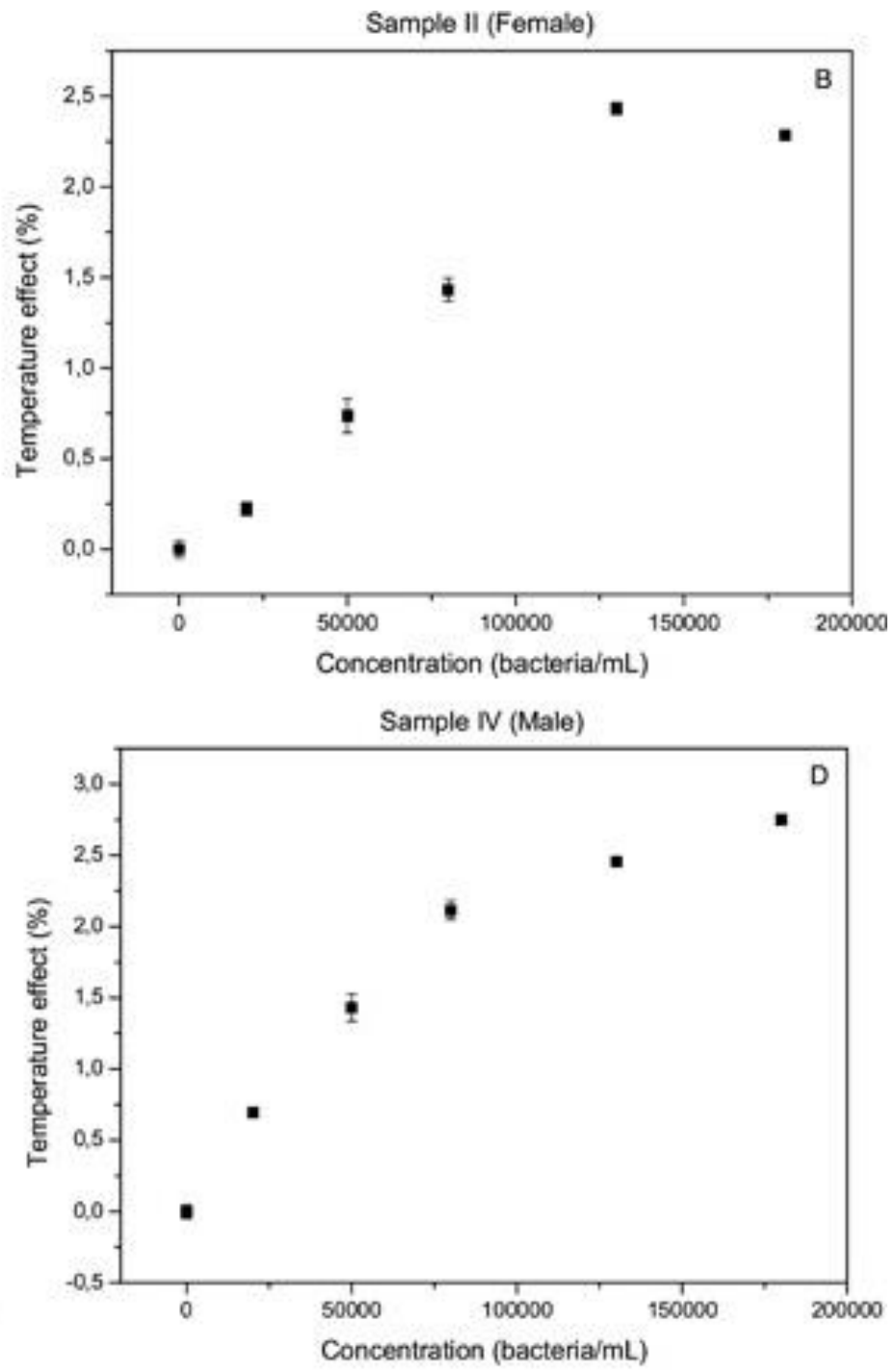

Figure 29 (b) and (d) - The results from these experiments were used to calculate dose-response curves. 


\subsubsection{Reproducibility of Dynamic Sensor Range}

The study was expanded by collecting and sterilizing urine samples of two healthy male and two healthy female individuals and spiking them with the aforementioned concentrations. The data were analyzed in a similar manner and three samples were analyzed for each of the test subjects (sample I to IV representing the four different test subjects). The results were obtained to construct a dose-response curve for each of the individuals under study (Figure 30). The data were fitted with a standard sigmoidal dose-response curve fit in Origin Lab (obtained $\mathrm{R}^{2}$ values were 0.96, 0.93, 0.99, and 0.98 for sample I-IV, respectively). The data indicate that the SIP-based bacteria sensor is largely independent on gender, age, and osmolality as all samples illustrate a very similar behavior with LoDs, calculated using the $3 \sigma$ method, 25 around $2-3 \times 10^{4}$ bacteria $\mathrm{mL}^{-1}$ and a linear regime until the response saturates at concentrations above $10 \times 10^{4}$ bacteria $\mathrm{mL}^{-1}$. 


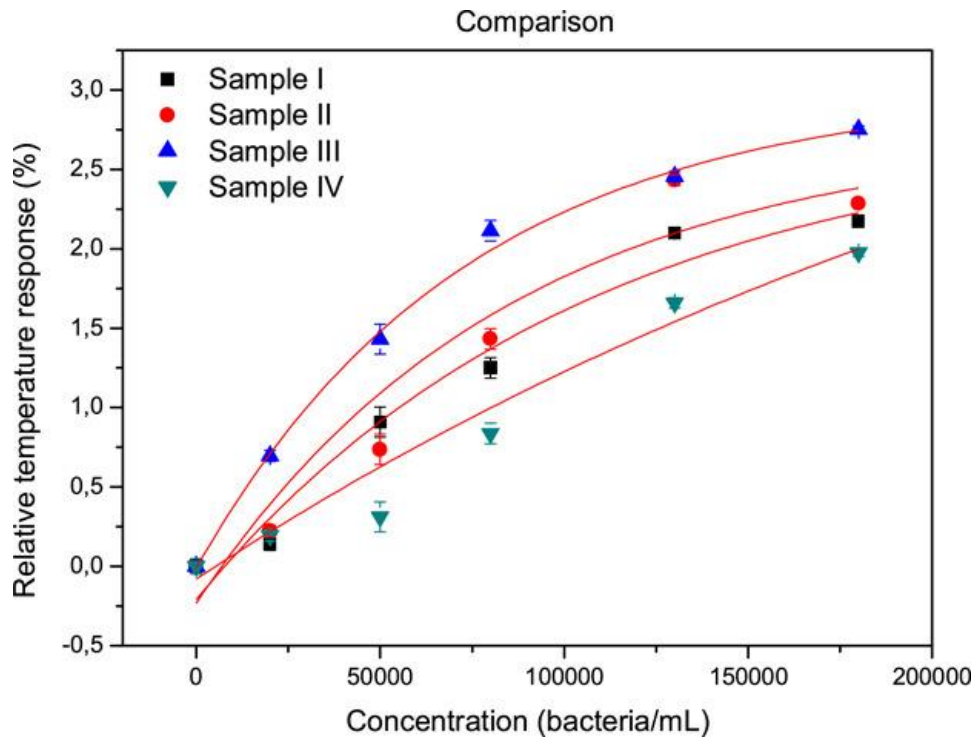

Figure 30 Dose-response curves of the time-dependent temperature data obtained in measurements using E. coli-SIPs. The relative response is plotted against the spiked concentration and a sigmoidal fit is used. Each curve (labeled sample I to IV) represents measurements on samples obtained from different volunteers.

\subsubsection{Potential Effects of Sample Storage and Transport} In order to examine the effect of transport of samples and delayed sample analysis, a spiked dilution series in urine was kept for $24 \mathrm{~h}$ prior to analysis. The dilution series was stored on room temperature for the duration of the previous measurement, transferred to a Styrofoam box typically used for sample transport for storage overnight and finally stored in the fridge for $2 \mathrm{~h}$ prior to thermal analysis. The measurement was performed analogously to the data presented in the previous section and a dose-response curve was constructed and compared to the data obtained in the previous section (Figure 31). Surprisingly, the bacterial colony already reached the 
decline phase with a significant portion of the bacteria dying, [26] leading to a decrease in the average signal at each concentration. In addition, the noise on the signal appears to be higher further narrowing the dynamic range of the platform and decreasing the sensitivity of the methodology.

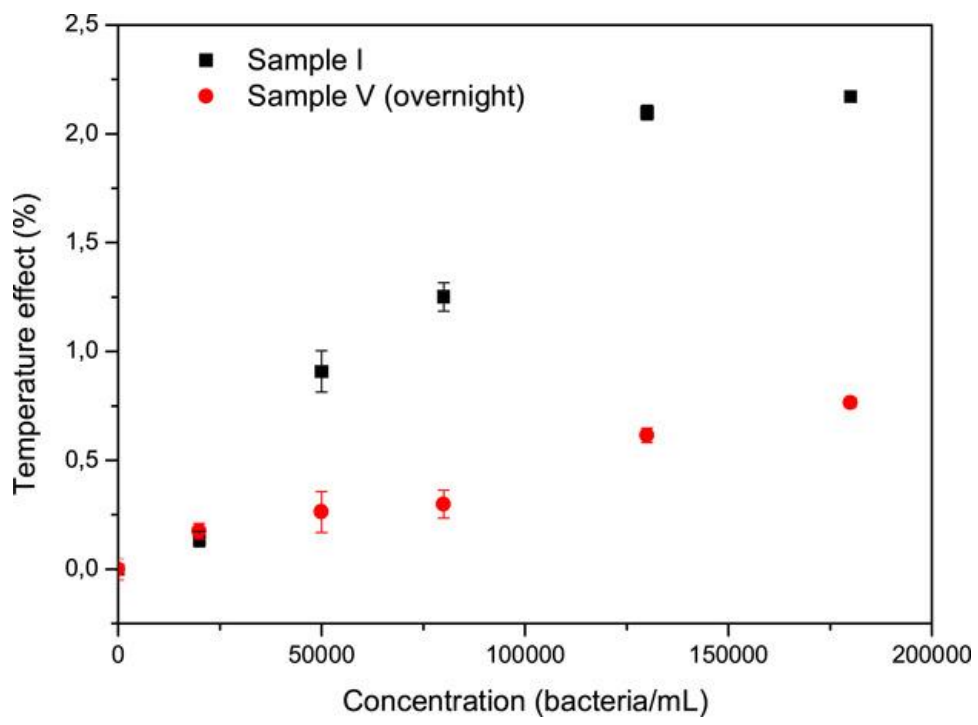

Figure 31 - Comparison of the dose-response curves obtained with samples that were properly stored and measured (Sample I) and sample V, which represents measurements on samples that were stored for a day on different temperatures, simulating realistic conditions in current practice.

\subsection{Discussion}

The results shown in this paper confirm the potential of the SIPbased thermal biosensor for application in UTI diagnosis. The horizontal dimensions and morphological characteristics of the bacterial templates are transferred to the SIP layer during imprinting (Figure 28). This process plays an essential role in 
the recognition process and results in highly specific microcavities that are capable of rebinding their target in a selective manner. The presence of some larger imprints, attributed to aggregate formation on the stamp surface, as well as the limited surface coverage indicates that the imprinting approach can still be improved in the future. The results in Figure 29 are in line with previously obtained data. The amount of bacteria that bind to the surface increases gradually when exposing a SIP layer to an increasing layer of bacteria in urine. This can be observed by the fact that the thermal resistance increases as demonstrated by the drop in $T_{2}$ in the timedependent temperature graphs.

When comparing the female to the male sample no apparent difference in effect size, dynamic range, or limit-of-detection can be observed. These findings are further confirmed by similar experiments on a larger sample size (Figure 30). This is a very important finding in terms of potential application of the sensing principle in point-of-care diagnostics. Urine consists of varying amounts of inorganic salts and organic compounds including proteins, hormones, and numerous other metabolites containing carboxyl or amino groups [27]. The concentration of these components can be affected by multiple parameters including, for example, the amount of water consumption, since the kidney excretes more or less dilute urine, changing its osmolality [28]. Moreover, it has been found that dietary and cultural dissimilarities can lead to significant differences in endogenous urinary profiles [29]. Likewise, hormonal differences between genders, namely the human chorionic gonadotropin (hCG), were previously found to be more abundant in females [30]. This causes variability of the concentration of compounds in different urine samples, which can have a detrimental effect on various sensor platforms based 
on, for example, electrochemical readout methods. Previous results have already indicated that the complexity of a urine sample has only a small effect on the effect size of the thermal measurement signal but results in a slightly lowered sensitivity due to the increased amount of noise on the measurement signal.[1] The results obtained in this study confirm these findings as only a limited amount inter-sample variability is observed on the effect size upon binding and only a small effect on the LoD and dynamic range can be observed due to small differences in the average amount of noise on the signal. A possible explanation can be attributed by the fact that the morphological complementarity between the target bacteria and the imprints is assisted by a functional match mainly based on hydrogen bonding $[31,32]$. The differentially present substances, such as proteins, hormones and ions in urine seem to only marginally interfere with the process of hydrogen bonding and therefore similar effect sizes are observed.

An additional benefit of the current methodology is demonstrated in Figure 31. The sensor seems to react to a far lesser extent to a sample that was stored at different temperatures for $24 \mathrm{~h}$. Although this effect could probably be minimized by proper and stringent handling of the samples, this is also not always the case in every day practice. Although uropathogenic bacteria have developed mechanisms to circumvent the antimicrobial functioning of urine and tend to grow in samples that are not stored, [33] a sharp decrease in the number of viable cells was encountered in this study. A potential explanation could lie in the fact that the lab strain used in this study is non-pathogenic and will therefore not be able to survive for prolonged periods without the presence of nutrients at room temperature [34]. Further investigation about the nature of the observed phenomena in follow-up studies could 
deliver some more insight into the mechanisms of this observation but the current experiment already illustrates that inappropriate handling and storing of samples could compromise correct interpretation of the data. This can, in turn, result in misdiagnosis in patients and increase in the amount of false negative in a diagnostic test. The current methodology can be used on the bench and upon optimization and calibration of the current method, should be able to provide the user with an outcome within less than an hour. In this way, the risk of false negatives due to long-term storage and transport becomes increasingly smaller.

\subsection{Conclusion}

The results presented in this article further confirm the diagnostic potential of the presented sensor platform based on the combination of highly selective synthetic receptors and an elegant, low-cost readout platform such as the thermal platform that was previously introduced by the authors. The sensor is able to detect bacteria in urine samples in concentration that are relevant for UTI diagnosis. The effect size, LoD, and dynamic range appear to be similar for different samples obtained from several healthy volunteers. There appears to be no discernible distinction between samples collected from males and females, regardless of the hormonal difference or the varying degrees of urine osmolality. In addition, the fast nature of the readout technology enables the end-user to overcome potential false negative results arising from long-term sample storage and transport which is typically associated with traditional urine sample analysis techniques that need to be performed in specialized labs. 


\subsection{Transition}

In this chapter, the utility of the HTM as a readout system for UTI was presented. The HTM is an inherently affordable technology, requiring only a heater and two thermometers in its base configuration. These attributes make the technology highly attractive for PoC use in LIC. Furthermore, the importance of fast sample analysis was shown, additionally proving the arguments made in chapter 2 . Therefore, the sensing system presented in this chapter, consisting of a SIP as a recognition element together with the HTM as a transduction and readout method, has the potential to impact diagnostics in LIC in a positive manner. However, as was laid out in the beginning of the thesis, recognition element and transducer make a biosensor, which is at the heart of a PoC device, but it is not a complete PoC device itself. To create a complete device we also need an appropriate way to manage the sample and transport it to the sensor, which can be achieved with a microfluidic setup. Therefore, the next chapters will focus on microfluidics, which will enable us to discuss a complete PoC device instead of just a sensor system. However, constructing microfluidics can be a considerably expensive undertaking, as traditional methods require a cleanroom. To avoid this, we will investigate $3 \mathrm{D}$ printing as a valuable low-cost alternative for microfluidic construction in LIC. 


\section{The Liberalization of Microfluidics}

Form 2 Benchtop 3D Printing as an Affordable Alternative to Established Manufacturing Methods

Adapted from:

B. Heidt, R. Rogosic, S. Bonni, J. Passariello-Jansen, D.

Dimech, J. W. Lowdon, R. Arreguin-Campos, E. Steen

Redeker, K. Eersels, H. Diliën, B. van Grinsven, T. J. Cleij,

Phys. Status Solidi A. 2020 217, 1900935, DOI:

10.1002/pssa.201900935. 


\subsection{Chapter Context}

In chapter 2 it was discussed that bringing healthcare access to LIC also faces problems regarding different design philosophies between HIC and LIC, as well as the problematic distribution infrastructure in LIC. A possible solution to solve this problem is proposed in this chapter, which concerns itself with the optimization of 3D printed microfluidic channels that can be used for integrated PoC device construction, using common and widely available 3D printer and resins. The benefits of microfluidic construction using 3D printing are that no cleanroom facilities are necessary for research and manufacturing; as well as the wide range of form factors that can be produced such that, for example, the microfluidic structure can be produced at the same time with the device housing itself, speeding up manufacturing; furthermore it can be used 'on demand', removing the necessity of large distribution infrastructure or extensive stock management for parts that were produced elsewhere. Commonly available printers and resins where chosen so that the results can be easily applied by other research groups.

\subsection{Introduction}

In the last two decades, 3D printing has revolutionized the world of prototyping and manufacturing. 3D printing in principle is a technology similar to classical 2D ink printing, but instead of ink on paper, it deposits multiple layers of a particular material one on top of the other, allowing for the creation of 3D objects [1]. Multiple types of technology are used in $3 \mathrm{D}$ printing, everything that involves the creation of objects by multilayer deposition, falls under the definition of additive manufacturing (AM). The huge developments of the last decades enable the use of a broad range of materials, each 
optimized for specific types of 3D printing [2, 3]. The most widely known and used techniques today are by far fused deposition modeling (FDM) and vat polymerization (VAT) also known as resin printing. These two types of AM technologies have experienced a huge commercial success: countless companies offer relatively cheap (available for private users) machines that have compact dimensions and are easily used to produce everyday objects. Due to the drive of the market, the advancements in technology allow these machines to reach high levels of precision today. Even in laboratory environments, researchers are more keen to use these devices in everyday activities, as 3D printing helps to develop custommade and tailored assays [4] or laboratory devices [5]. FDM and VAT are based on very different technologies: in an FDM printer, a polymeric filament is extruded after being heated at an appropriate temperature. The extruded material is deposited in a thin layer on a building plate and the following layers are stacked to form the final object (Figure 32). FDM technology is in principle very simple and allows for the use of a variety of thermoplastic materials that can be extruded through a heated nozzle.
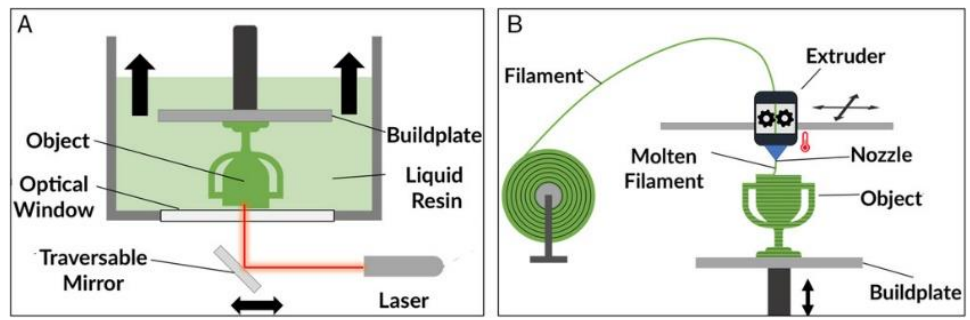

Figure 32 - A) VAT printing principle and B) FDM technology principle. 
Due to its easy printing process and reliability, the most commonly used material in FDM is poly lactic acid (PLA). Other materials, such as acrylonitrile butadiene styrene (ABS), poly propylene (PP) and poly carbonate (PC), can be used as well [6]. The main advantages of FDM printing are the low cost of the material and the straightforward printing process. Generally, spools of plastic filament are directly fed into the printer. No processing is needed once the print is done and the printer itself needs very low maintenance as the modern designs are rugged. FDM might therefore seem the perfect printing process; however, there is a main disadvantage: the surface smoothness of the final object is lower when compared with other AM techniques (Figure 33). This is intrinsic to the layer-per-layer melting, deposition, and cooling process. While the discretization of an object introduces approximations such as stair-stepping for all AM techniques, this is particularly evident in FDM printers, due to the relatively high layer thickness. It is possible to smoothen the surface of a FDM print, for example, by sanding or with acetone vapour at an expense of time and dimensional precision of the printed part. On the contrary, VAT techniques have layers with reduced thicknesses, limiting this effect. The material used for the creation of the object is a liquid resin, usually an acrylate $[7,8]$ and it is photopolymerized at near-room temperature. The final result is a smoother and highly detailed object. The higher spatial resolution obtainable with VAT printers is also due to the smaller polymerization spot: while FDM printers have nozzles that are usually in the range of $250-500 \mu \mathrm{m}$, VAT printers use light sources, either projection screens or lasers, with a polymerization spot size ranging from 30 to $150 \mu \mathrm{m}$. VAT printers consist of a moving build platform that is immersed in the resin tank. The build platform is precisely positioned with respect to the bottom of the resin tank, to leave 
exactly the amount of resin necessary to cure a single layer. After the light source polymerizes one layer, the build platform is moved upward by the same height of the layer and then the next layer is cured (Figure 32). This process is repeated for each layer. As for FDM printing, commercially available modern resin printers are well optimized and allow for high-accuracy prints in a range of materials with various properties [9]. VAT printers can be divided in two categories: stereolithography (SLA) and digital light processing (DLP) printers. The difference lies in the light source used: SLA printers rely on a laser that, through a series of mirrors, focuses the light at the appropriate planar coordinates on the build plate. DLP printers use a digital projector screen as the light source, projecting therefore the whole image of the layer and curing all the point simultaneously.

A

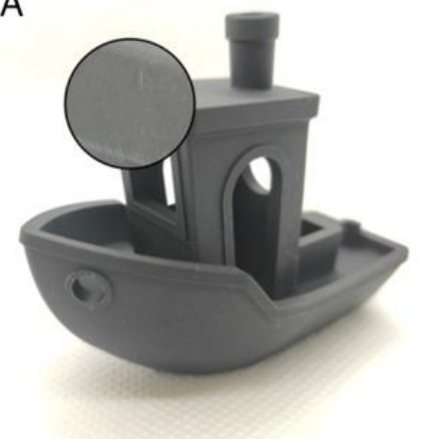

B

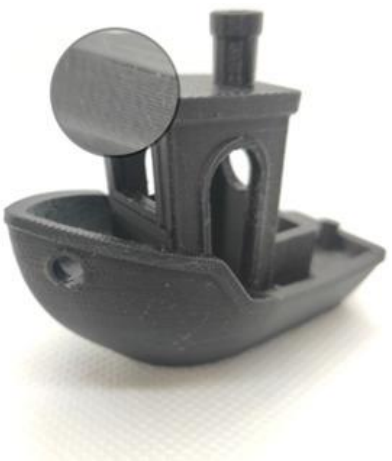

Figure 33 - A) Object printed with the Form 2 SLA printer (layer thickness $50 \mu \mathrm{m})$ : the final part is much more detailed and the surface finish is smoother if compared with B) the same object printed with an FDM (Ultimaker 3, layer thickness $100 \mu \mathrm{m}$ ) printer. In the magnification, the stairstepping lines are well visible. 


\subsubsection{D Printed Microfluidics}

Due to the well-known advantages of microfluidics in many laboratory applications,[10] research in this field developed substantially in the last 10 years. The vast majority of microfluidic chips found in the literature are fabricated by lithography techniques;[11] however, in recent years, with the development of 3D printing, there are commercially available machines that allow for the fabrication of microfluidic devices with AM techniques [12]. The main advantage is the one-step process that reduces the time needed for fabrication [13]. A contribution to the recent development of $3 \mathrm{D}$ printed microfluidic devices comes from the availability of biocompatible materials, which allow researchers to use their devices in biological applications $[14,15]$. In this field, SLA is a commonly used technology: in 2014, researchers printed a 3D immunomagnetic flow assay [5]. The device was used in combination with magnetic nanoparticles and antibodies, allowing for the separation of Salmonella bacteria. Similarly, another group used SLA printers to fabricate a micro-mixer, a gradient generator, a droplet extractor, and a device for isotachophoresis [16]. This work showed how such structures can be obtained in a single fabrication step using SLA technology. In a later work [17] researchers managed to print in a single step, designs with integrated valves, controlled by pneumatic actuation. All these examples illustrate the potential of SLA printers in the further development of 3D printed microfluidics. The higher accuracy and surface definition of this technique are the main advantages that made commercially available SLA printers the choice for the majority of researchers interested in 3D printing of microfluidic devices. 


\subsubsection{Accuracy in 3D Printing}

Comparing the accuracy of two resin printers only by looking at the technical specifications is a difficult task. Many factors such as materials, software settings, and postprocessing contribute to the quality of the printed object. Resolution in the $\mathrm{Z}$-axis is often used as a selling point by manufacturer to attract attention; however, in resin printers, $\mathrm{XY}$ resolution is most of the times the limiting factor in achievable feature size. Zresolution is the minimum distance that the build plate can travel in the Z-direction and corresponds to the layer thickness. The XY resolution depends on the light source used to cure the resin: in the Form 2 printer, the minimum feature size on the $\mathrm{XY}$ axis is $140 \mu \mathrm{m}$ and corresponds to the size of the laser spot that is projected onto the build plate.

\subsubsection{Benchmark of the Form 2 SLA Printer}

The majority of literature on 3D printed microfluidics use their own 3D printed setup or develop their own resin, optimized for the selected printer. This can result in difficulties when trying to replicate their work, limiting the knowledge transfer potential. In this work, we focus on a commercially wellestablished SLA printer that has one of the best quality-price ratios on the market: the Form 2 from Formlabs. The Form 2 printer can be used with a variety of different resins that are tuned for specific properties. In this work we characterize the printing quality of the Form 2 printer in microfluidic applications, in terms of surface finish, accuracy (deviation from nominal size), and optical properties. We show how the printed objects are influenced by different printing orientations on the build plate as well as how the final result is affected by the different resin used (among three resins). We selected to test Clear resin, Tough resin, and High Temperature resin as they are the ones most commonly used in laboratory 
environments due to their specific advantages (transparency, resistance to temperature, and good mechanical properties, respectively).

\subsection{Experimental Section}

The resins used were the following: Clear resin V4(FLGPCL04), Tough resin V5(FLTOTL05), and High Temperature resin V2(FLHTAM02). All the resins were purchased from Formlabs. Preform 3.0.1 (Formalbs) software was used as slicer and for every print was set to a layer thickness of $50 \mu \mathrm{m}$. The test objects were designed using Fusion 360 (Version 2.0.6, Autodesk Inc.).

\subsubsection{Precision}

The goal was to highlight the influence of resin type and inclination of the printed object, on the final result. The printer printed the object layer by layer: the planar projection at a defined height corresponded to the layer being printed at that height. The inclination of the object had different effects: as shown in Figure 34A, varying the inclination of an object on the build plate modifies the surface area of each layer. A direct effect was the modification of the force acting on the object as it is detached from the resin tank after the polymerisation of each layer. A higher surface area created higher stresses acting on the object. On the contrary, higher areas mean that the laser was able to reproduce with higher accuracy a very small feature (Figure 34B). In objects with specific irregular and small features, this technique can be exploited to obtain higher quality details. A Leica S6E optical microscope with a 40x times magnification, equipped with a Leica DFC290 HD digital 
camera, was used for all the photos and the prints were aligned to account for the viewing angle. The images were analysed with the software ImageJ (Version 1.8.0, imagej.nih.gov/ij/download.html), each channel/feature was measured 3 times to obtain an average. Accuracy (in \%) was defined as the ratio between the nominal size and the measured size multiplied by 100 .
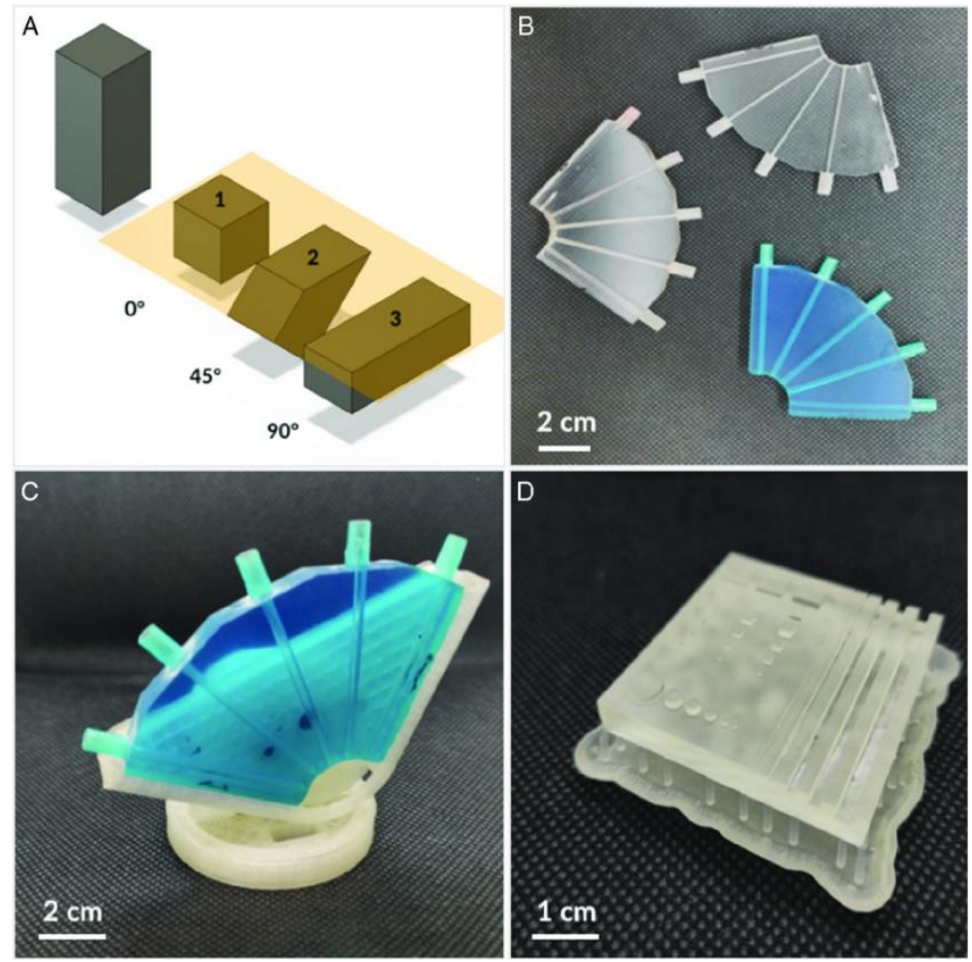

Figure 34 - A) Printing object in different orientations has important effects on the end result. B) Test objects printed with different dimensions and orientations. C) Orientable stand for photoacquisition. D) Open channel features. 


\subsubsection{Embedded Channel Accuracy}

A test object (Figure 34A) was printed, featuring a single squared embedded channel oriented at five different angles, equally distanced between $0^{\circ}$ and $90^{\circ}$ with regard to the build plate. The test object, as shown in Figure 34, is a quarter disk. For each resin, we printed four quarter disks with channel size of $0.5,1,1.5$, and $2 \mathrm{~mm}$. In total, we printed and tested 12 test objects.

\subsubsection{Open Feature Accuracy}

To test the accuracy of features printed on the outer part of an object, a test object featuring various geometrical shapes was printed. As shown in Figure 34D, the test object includes open channels, circular shapes, and squares in different size and height configurations.

\subsubsection{Optical Characteristics}

Optical transparency was important in microfluidics as it allowed to observe the correct functioning of the device, discovery of disturbances like bubbles, and eventually for the use of optical readout elements. To evaluate the optical properties of the three different resins, a simple $3 \mathrm{~mm}$-thick window adapted to be compatible with a Shimadzu UV-1800 UV spectrophotometer was printed. The complete visible spectrum from 380 to $740 \mathrm{~nm}$ was measured in $1 \mathrm{~nm}$ increments. To improve the optical properties, the part was wet sanded in increasingly fine grain size $(400,600,1000,2000)$ and coated with Motip effect clear varnish and the spectrum measurement repeated. 


\subsection{Results}

\subsubsection{Open Channels}

Open channels proved to be less difficult to produce than closed channels, as shown in Figure 35. The width of channels for the High Temperature resin and the Clear resin was over $90 \%$ accurate down to a channel size of $0.5 \mathrm{~mm}$. The Tough resin, however, only managed over $90 \%$ width accuracy down to $1.5 \mathrm{~mm}$ and then slowly declined further until reaching an accuracy of $73.19 \%$ at $0.25 \mathrm{~mm}$ channel size. The lowest channel accuracy, however, showed the clear resin at $0.25 \mathrm{~mm}$ with $58.97 \%$. For depth accuracy, the Clear resin and High Temperature resin achieved even better results, retaining over $90 \%$ accuracy within all tested channel depth. The Tough resin proved to be less accurate in depth as well, showing over $90 \%$ accuracy only at $2 \mathrm{~mm}$; however, 1.5 and $1 \mathrm{~mm}$ trailing close to $90 \%$ with $89.22 \%$ and $88.56 \%$, respectively. Only then follows a strong decline to $71.93 \%$ at $0.5 \mathrm{~mm}$, before it ends with the lowest open channel value of $21.12 \%$. The Clear resin and High Temperature resin could still produce open channels without much trouble at half the size of their closed channels lowest point. 


\section{The Liberalization of Microfluidics}
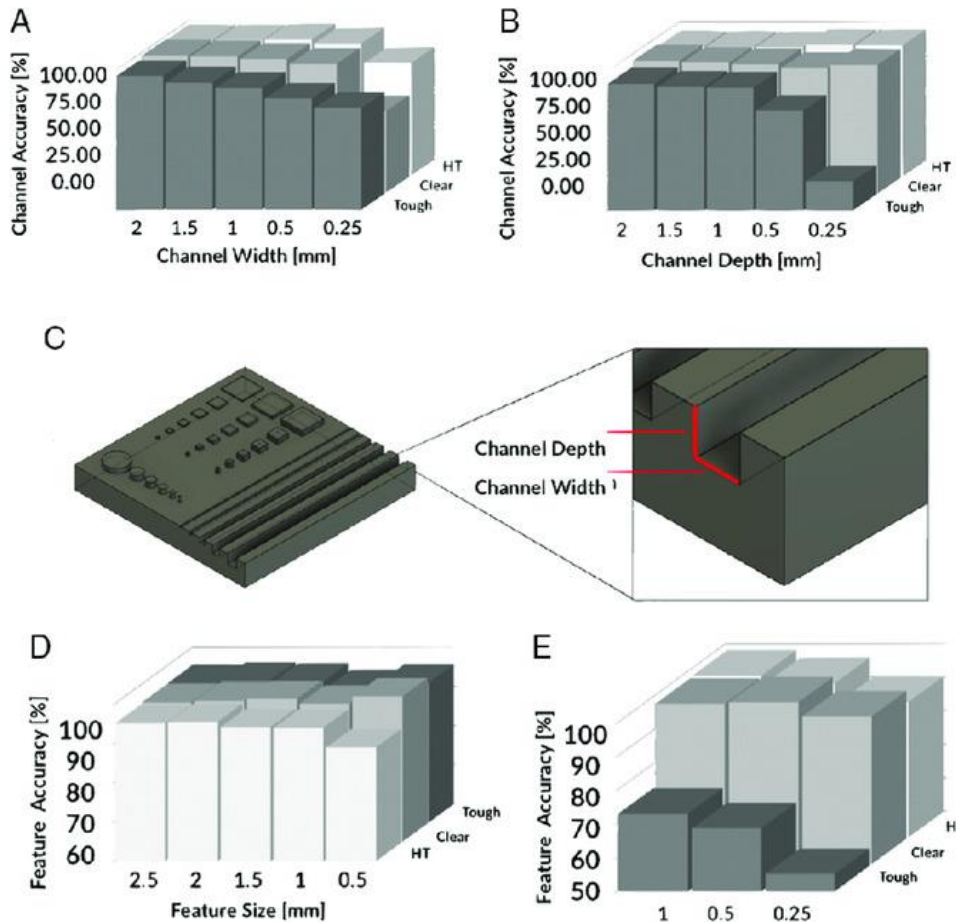

$\mathrm{F}$
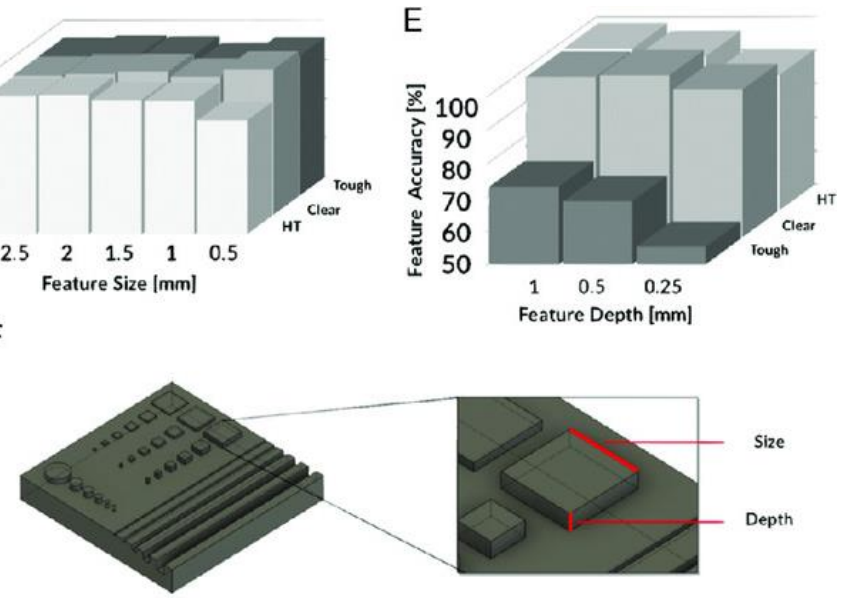

Figure 35 - Accuracy of open channel prints. A) Width accuracy of the three different resins for five channel width. B) Depth accuracy of the three resins in five different depths. C) Rendering of the printed part with indicated measuring area. D) Size accuracy of five different square sizes with the three different resin types. E) Depth accuracy of the squares for three different height types. F) Rendering of the test structure with marked areas of measurement. 


\subsubsection{Features}

The feature size accuracy was good throughout each resin and feature size as seen in Figure 35. Every sample reached over $90 \%$ accuracy except the square with a length of $0.5 \mathrm{~mm}$ printed with High Temperature resin, which closely failed the $90 \%$ mark with $89.08 \%$. Interestingly, the highest accuracy was achieved by the Clear resin at the smallest feature $(0.5 \mathrm{~mm})$. In general, a feature size of $1.5 \mathrm{~mm}$ showed to be most accurate with most resins. While the performance of the Clear resin and Heat Resistant resin in the depth accuracy was satisfactory, reaching over $90 \%$ in every size increment, the Tough resin showed to have troubles, reaching its best mark at $1 \mathrm{~mm}$ with only $73 \%$ and its lowest at $0.25 \%$ with only $55.2 \%$ accuracy.

\subsubsection{Closed Channels}

As shown in Figure 36, there was a clear slope in performance visible in all resins for the production of internal channels. In general, the best performance was observed with larger channel size as well as higher degree of angle. This tendency is especially visible in the clear resin, where the largest vertical channel $\left(2 \mathrm{~mm}\right.$ at $\left.90^{\circ}\right)$ showed the highest accuracy $(96.45 \%)$, whereas the smallest channel and the smallest angles $(0.5 \mathrm{~mm}$ and $0^{\circ}$ ) could either not be printed at all, or had the lowest accuracy. This effect was less pronounced with the High Temperature resin and Tough resin. Wide channels $(2 \mathrm{~mm})$ could be developed with every resin in every angle. On the contrary, only the Tough resin was able to print the smallest channel $(0.5 \mathrm{~mm})$ down to $22.5^{\circ}$ and only failed at a horizontal print of the smallest channel $\left(0.5 \mathrm{~mm}\right.$ at $\left.0^{\circ}\right)$. The Clear resin reached over $90 \%$ accuracy at an angle of $90^{\circ}$ with $2,1.5$, and 1 large channels. At $67.5^{\circ}$ it achieved over $90 \%$ accuracy with only 2 and $1.5 \mathrm{~mm}$ and at $45^{\circ}$ only with $2 \mathrm{~mm}$ channel size. At $0^{\circ}$ it was only able to print channels of $2 \mathrm{~mm}$ size. Channels 
with a size of $0.5 \mathrm{~mm}$ could not be printed at all with the Clear resin, independent of the angle. The High Temperature resin performed better, reaching over $90 \%$ accuracy at $90^{\circ}$ with 2 , 1.5 , and 1 channel size and at $67.5^{\circ}$ with $2,2.5$, and $1 \mathrm{~mm}$ channel size. It failed to produce channels at $0^{\circ}$ from 0.5 to $1.5 \mathrm{~mm}$ and channels with size of $0.5 \mathrm{~mm}$ up to a slope with $46^{\circ}$. The Tough resin performed best with over $90 \%$ accuracy at: $90^{\circ}$ and channel sizes $0.5,1,1.5$, and $2 \mathrm{~mm} ; 67.5^{\circ}$ and channel sizes of $1,1.5$, and $2 \mathrm{~mm} ; 45^{\circ}$ and channel sizes of 1 , 1.5 , and $2 \mathrm{~mm} ; 22.5^{\circ}$ and $2 \mathrm{~mm}$ channel size. It only failed to produce channels at $0^{\circ}$ and $0.5 \mathrm{~mm}$ channel size.

A

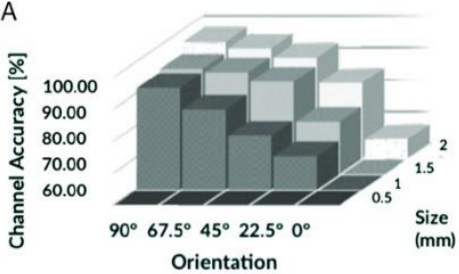

C

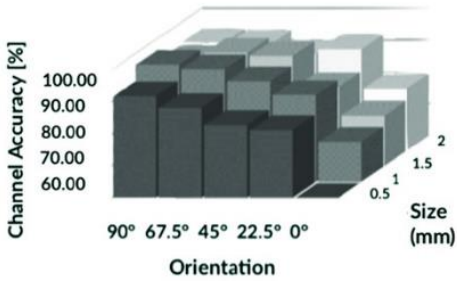

B

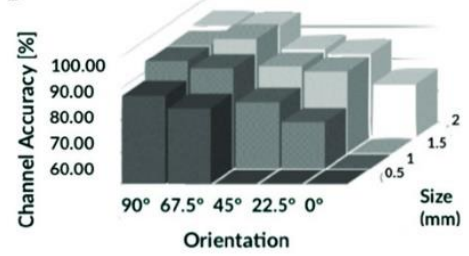

D

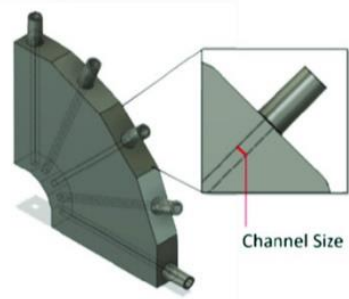

Figure 36 - Accuracy of $3 D$ test prints with internal channels in four different channel sizes and five different angles. A) Clear resin, B) High Temperature resin, C) Tough resin, and D) rendering of the test model.

\subsubsection{Optical Characteristics}

As shown in Figure 37A, in the visible light range the High Temperature resin started with the largest transmission of light at $30.9 \%$, followed by the Clear resin with $16.93 \%$ and the Tough resin with $12.36 \%$. After the surface treatment process, 
the Clear resin showed the best properties with $73.18 \%$ transmission, followed by the High Temperature resin with $71.33 \%$ transmission. The Tough resin performed the poorest with only $38.8 \%$ transmission. This is due to two absorption maxima at 616 and $710 \mathrm{~nm}$ (Figure S1, Supporting Information) leading to the resins intense blue color. As the surface treatment only decreases light scattering, the Tough resin does not profit from this effect as much as the High Temperature and Clear resin, whose low initial transmission is mainly due to scattering. However, as shown in Figure 37B, the surface treatment shows the same effect in diminishing the light scattering from the surface.
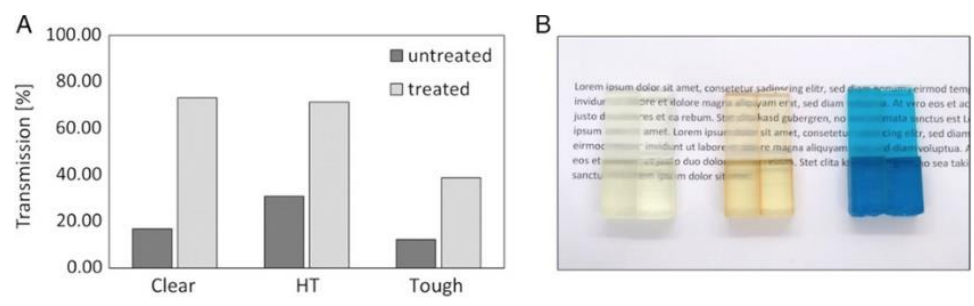

Figure 37 - A) Transmission values for the three different resins before and after the surface modification process. B) Comparison of transparency for the three different resin types before (left) and after (right) the surface modification, from left to right: : Clear, High Temperature, and Tough resin.

\subsection{Discussion}

\subsubsection{Performance of Different Resin Types}

The Clear resin did perform the worst of the three resin types for internal channels. This is surprising as it was assumed that the more specialized High Temperature and Tough resins would perform worse as they are fine-tuned for different purposes compared with the Clear resin, which is used more as an all-rounder. As expected, larger channels, printed at more vertical angles, show the best accuracy. The reason for this 
effect is most likely the entrapment of uncured resin in the closed channels. With lower channel size and angle, the entrapped resin has less opportunity to flow out of the channel, and thus stays inside and gets partially cured by stray light of subsequent layer formation. This, in turn, leads to a reduced accuracy and in extreme cases, as with small dimensions and angles, to complete clogging. This hypothesis is also supported by the observation that channels usually get clogged from the middle (Figure S2A, Supporting Information). As described earlier, this can be attributed to the trapped resin that manages to escape on the sides but not in the middle. This also shows in the construction of open channels, which was more accurate and worked very well for most resin types. Interestingly, the Tough resin was here underperforming. A possible explanation might be the different curing properties of the Tough resin, which seems to have problems with surfaces that are flat to the build plate, the direction this model was printed. On the underside of those prints, malformed parts of cured resin can often be observed, which is not the case for High Temperature and Clear resin (Figure S2F, Supporting Information).

Regarding the open features, interestingly, the largest features did not perform best. However, the regularity of the shape was more accurate in the larger features and did tend to lose the perfect squared shape in the smaller samples (Figure S2E, Supporting Information).

Regarding the optical properties of the printed parts, the refined surface treatment method to improve the light transparency worked very well and was consistent with former experiments conducted by Formlabs.[19] 


\subsection{Conclusion}

Techniques such as 2-photon polymerization can boost precision in microfabrication to new limits; however, this advanced technology that requires expensive machines, which limits its use in everyday laboratory activity [20]. Lithography techniques remain the gold standard for laboratory applications such as cell sorting or single-cell analysis [21]. This is due to the low achievable feature size (comparable with the size of a cell), and the well-studied capabilities and reliability. Small channels have the advantage of faster chemical reaction time due to smaller average diffusion length, which makes them preferable for high efficiency microreactors [22]. In Bioreactors where, for example, yeast is used as an expression agent, subcellular structures are needed to hold the cells in place while still allowing for medium perfusion [23]. The low Reynolds number achieved with small channel sizes enables a laminar flow regime, which is essential for reliable separation of cells, for example, in flow cytometry and in cell-culture small feature size allows the creation of microenvironments, for example, to observe cell behavior in different conditions [24]. The aforementioned well-studied capabilities and reliability of the established lithography techniques are essential for successful application in the low microfluidic ranges as the channels are in a size regime where even minor variations will have large effects. The capabilities of benchtop $3 \mathrm{D}$ printers like the Form 2 are not yet comparable. This is due to the large difference in achievable channel size; however, often experimental setups do not necessitate such small channels $(1-50 \mu \mathrm{m})$ when used in biosensing applications [25, 26]. Channels size between 250 and $500 \mu \mathrm{m}$, despite being in the upper range of the microfluidic scale, are still very useful in manipulation of small volumes of fluids. Considering the 
inexpensiveness of such machines and their easiness of operation, there is a wide array of researchers that can benefit from the utilization of an SLA printer such as the Form 2 [27, 28]. We demonstrate the capabilities of this printer, highlighting the relation between accuracy, channel size, angle, and resin choice. Applying these findings in the fabrication of complex objects will help researchers tune their designs to increase the efficiency of their work.

\subsection{Transition}

With the results in this chapter the groundwork for reliable microfluidic 3D printing was laid, and this knowledge was used for the construction of new flow cells and microfluidic building blocks. As a demonstration of the actual application of the created knowledge, the next chapter will provide an overview of these applications. First, a new, 3D printed flow cell for the HTM will be presented and secondly, modular microfluidic building blocks, which were the basis for our spin-off company Flui.Go, will be shown. 


\section{3D Printed Microfluidics}

Applications in Research and Education 


\subsection{Chapter Context}

In chapter 2 it was discussed that many innovations made in the laboratory never find their way to the market. I had the incredible fortune that my research was different and lead to the creation of a spin-off company, even though in a different context than originally expected. This slightly different chapter therefore describes how the progress made in 3D printing lead to further improvements in our measuring setup and consecutively to a start-up company with a focus on education.

The chapter shows how a new and completely 3D printed flow cell for the HTM system was developed based on the findings obtained in previous chapters. The flow cell featured improved user friendliness, as it works without any screws to assemble the cell. For measurement with the old flow cell, the aluminumSIP-substrate had to be inserted and the flow cell closed via four screws at each corner. After this, the flow cell had to be arrested within a clamp, to prevent movement during measurement. Overall, the setup procedure took several minutes, this was especially cumbersome when air bubbles got trapped inside the measurement chamber, in which case the whole measuring setup had to be disassembled and reassembled. The new $3 \mathrm{D}$ printed system improved on the old version by making this process effortless as well as preventing bubble intrusion in the first place. The system was inspired by the LEGO building block system and parts fit together based on LEGO-like connectors. This enables easy assembly and disassembly of the flow cell as well as fastening of the flow cell onto a baseplate, furthermore several additional parts were printed such as an inclined ramp, and bubble trap to prevent airbubbles in the system. 
After realizing the potential of this modular and easily connectable system, a set of modular building blocks was designed; each block containing different microfluidic channels which serve different functions. While this was first planned as a biochip-project it rapidly found application in an educational context.

\subsection{D Printed HTM-Flow Cells}

The new flow cell consists of two parts; in the bottom part, the copper heater of the old flow cell can be inserted, while the upper part serves as the flow cell and lid. When both parts are attached together with the LEGO-like connection system, the top part will press the SIP onto the heater, arresting everything. With this, no screws were necessary to assemble the measurement device and it enabled quick insertion and extraction of the SIP-element. Furthermore, the underside of the bottom part also features the LEGO-like connection system. With this, the whole flow cell can be attached to a LEGO baseplate and no attachment inside a clamp is necessary. The system was further complemented with different additional parts. Among these was an inclined ramp which fits under the bottom-part and inclines the measurement setup so that flushing out of air-bubbles is made easier. Additionally, a bubble-trap was created that prevents air-bubbles from entering the system in the first place by using the buoyance of air-bubbles to trap them in a cul-de-sac in the adapter. The whole system connects together with 3D printed LUER-slide adapters, which can be arrested to the LEGO buildplate as well. Lastly, a waste container holder, fitting $50 \mathrm{~mL}$ Falcon-tubes, was used to have a dedicated waste-disposal container arrested 
on the buildplate, Figure 38 shows a rendering of the complete set and Figure 39 the printed and assembled setup.

The devices were printed with a FORM2 (Formlabs) and Clear Resin (V4) in $50 \mu \mathrm{m}$ layer height, after print it was washed for 20 minutes in a Formwash (Formlabs) with isopropanol. Channels were flushed with isopropanol to remove remaining uncured resin and the devices post-cured in a Formcure (Formlabs) for 20 minutes at $60^{\circ} \mathrm{C}$. Teflon tubing was used together with the adapters to be able to connect them to the sample-carrying syringes and use micropumps that were further away. However, direct injection with a syringe is also possible due to the LUER-adapters directly integrated in the flow cell. To increase transparency the top-side of the lid was wet-sanded with sanding paper with increasing fine granularity $(600,800,1000,2000)$ and two layers of clear lacquer were applied, resulting in a clear, transparent finish.

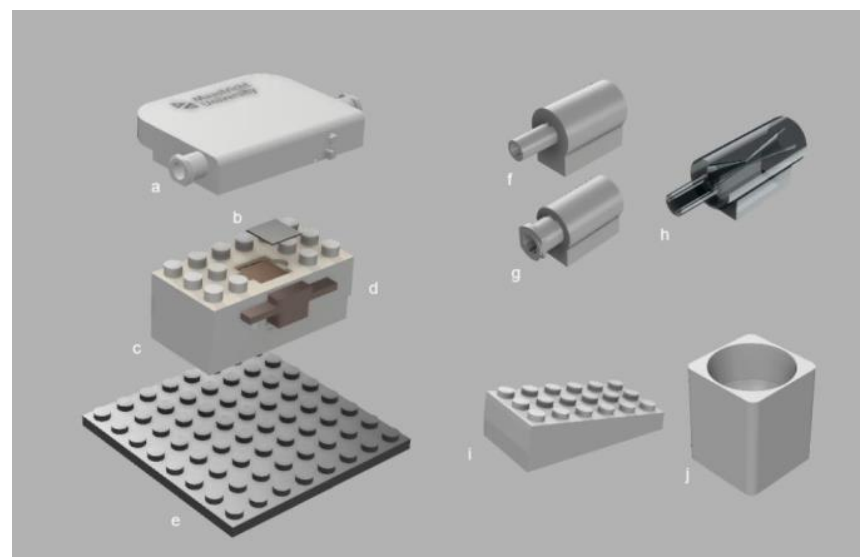

Figure 38 - Parts of the new flow cell concept. a. lid containing the flow cell, b. aluminum substrate, $c$. heater-holder, d. heater, e. LEGO baseplate, f and g. male and female LUER-to-tubing adapters, $h$. male LUER adapter with integrated bubble-trap, i. inclined ramp, $j$. holder for falcon tubes. 


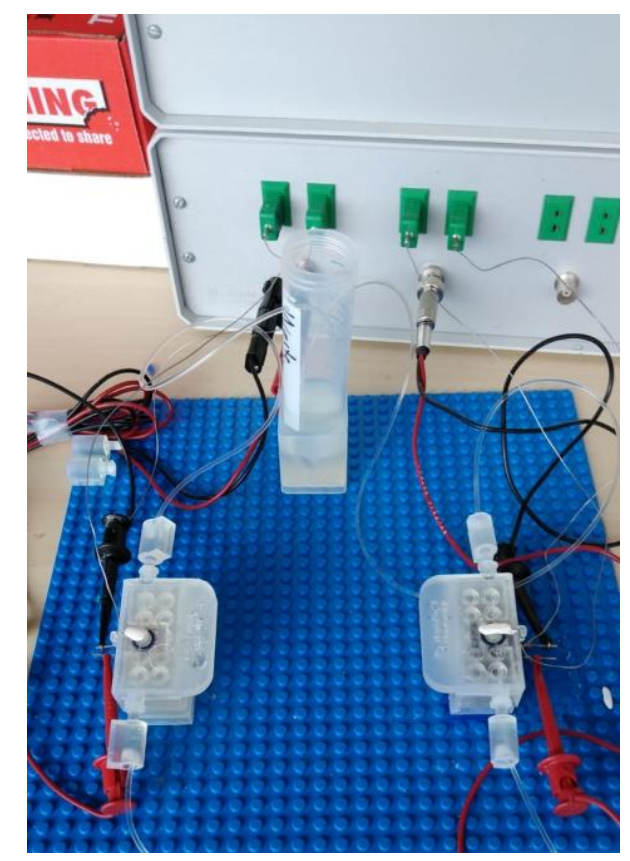

Figure 39 - Printed and assembled setup, arrested on a commercially available LEGO buildplate.

\subsection{Flui.Go}

After the successful design of the LEGO-inspired flow cells, the concept was taken further with the aim to create a completely 3D printable LEGO-inspired microfluidic brick system. Here the aim was to create different module-blocks that connect together easily and contain different integrated microfluidic channels, which serve different functions, for example a chamber for cell-growth, optical analysis, reactions, sample extraction and more. While it was learnt later that other research groups worked on similar ideas for modular 
microfluidics, like micro milling channels into existing construction blocks [1], 3D printing the whole block can be considered a strong improvement as it allows for truly threedimensional channel configurations and integrated adapter construction. A first generation of modular microfluidic bricks was created together with Maastricht University undergraduate students in a teaching project during the Maastricht Science Programme project period. Thereafter the faculty of Science and Engineering held a competition for science communication: 'Science + Engineering for Society'. STEM education was deemed to be a fitting use for the newly developed first generation of microfluidic bricks. Their use is intuitive and fun and thus could be exploited as a vehicle for STEM education, by developing easy experiments for students to perform by assembling the bricks. Therefore a second generation of microfluidic bricks was developed (Figure 40), together with several experiments for science communication and STEM-teaching. The discrete parts featured channel parts like straights, curves, T- and X-crossings and more (Figure 41) which can be assembled in a wide variety of different ways to perform experiments (Examples in Figure 42). This showed to be successful and the competition was won. After securing additional funding by the Universiteitsfonds Limburg (SWOL), further research could be conducted, and a third generation of microfluidic building blocks was constructed, together with a complete pumping system. With this now advanced setup, we managed to publish the results of this endeavor in the Journal of Chemical Education [2]. 


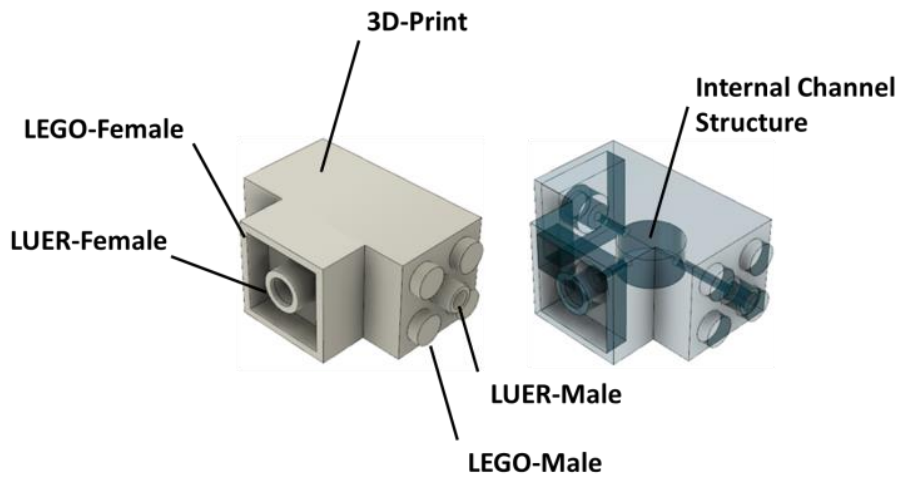

Figure 40 - Schematic of the second generation Flui.Go block 
3D Printed Microfluidics

1.

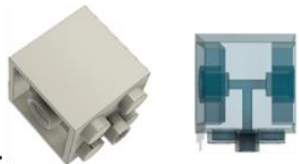

2.

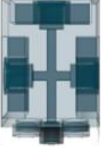

5.
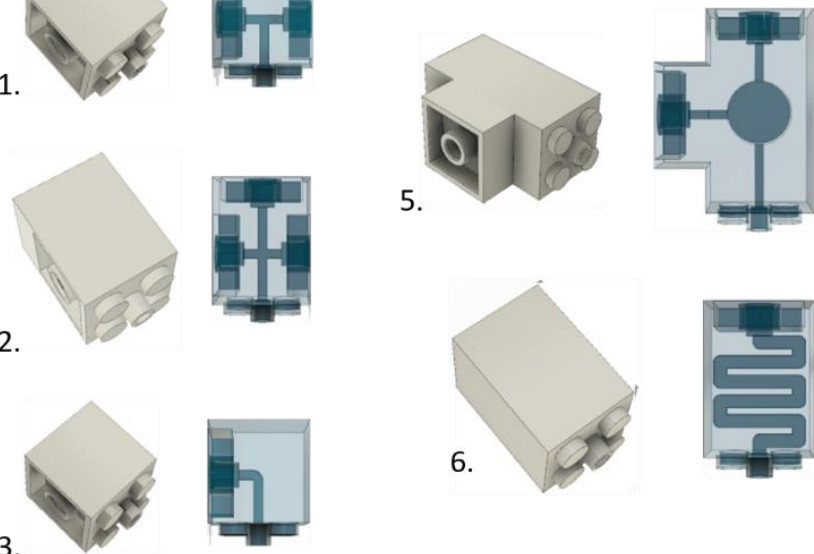

6.

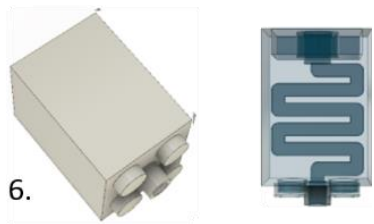

3.

4.
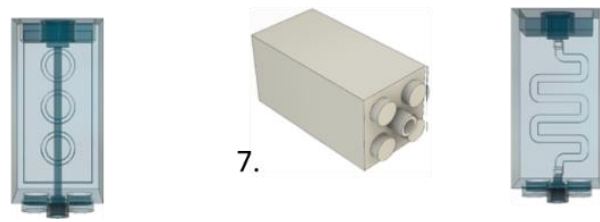

Figure 41 - Overview of the different microfluidic building blocks 1. TConnecter, 2. X-Connector, 3.Curve, 4 straight channel, 5. Reaction-block, 6. meander-mixer 7. Bubble expulsion.
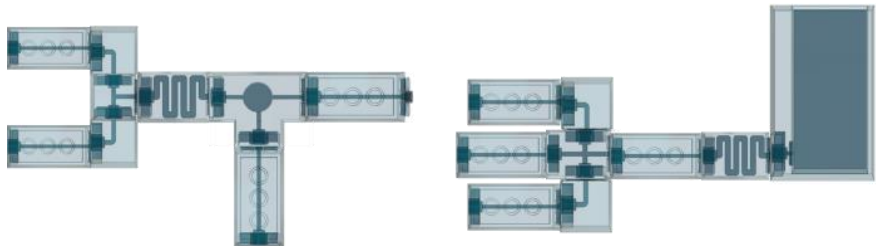

Figure 42 - Example of added microfluidic networks for experiments Left: 3 component reaction chamber. Right: Droplet generator with collection container. 
The manufacturing via 3D printing built strongly on the findings of chapter 4. Fusion 360 was used to design the parts and Preform to prepare (slice) the .STL files for printing. To ensure unclogged channels the parts were printed with a channel-angle of $>45^{\circ}$ towards the buildplate (Figure 43). A full-raft with 0.80 support density and $0,6 \mathrm{~mm}$ touchpoint size was the standard protocol for printing. Figure 44 shows bricks of the second and third generation as well as an experimental setup.
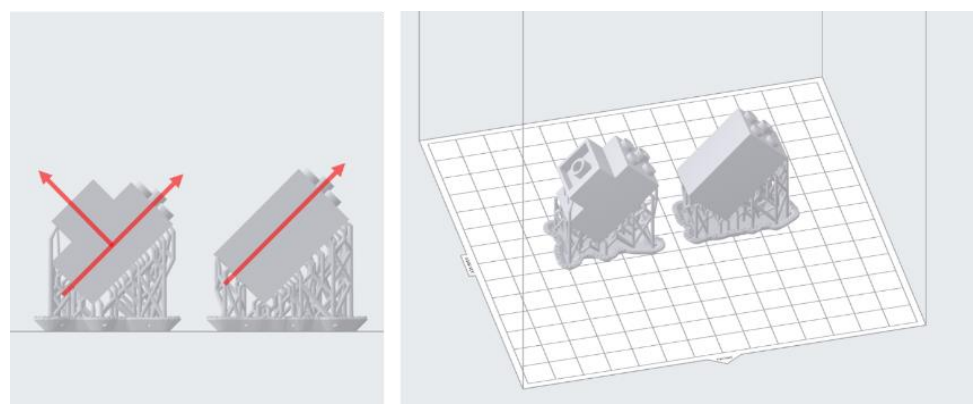

Figure 43-Print setup in Preform, Red arrows represent escape path for uncured resin through the channels (print is upside-down in bat configuration, red arrows pointing downwards). 


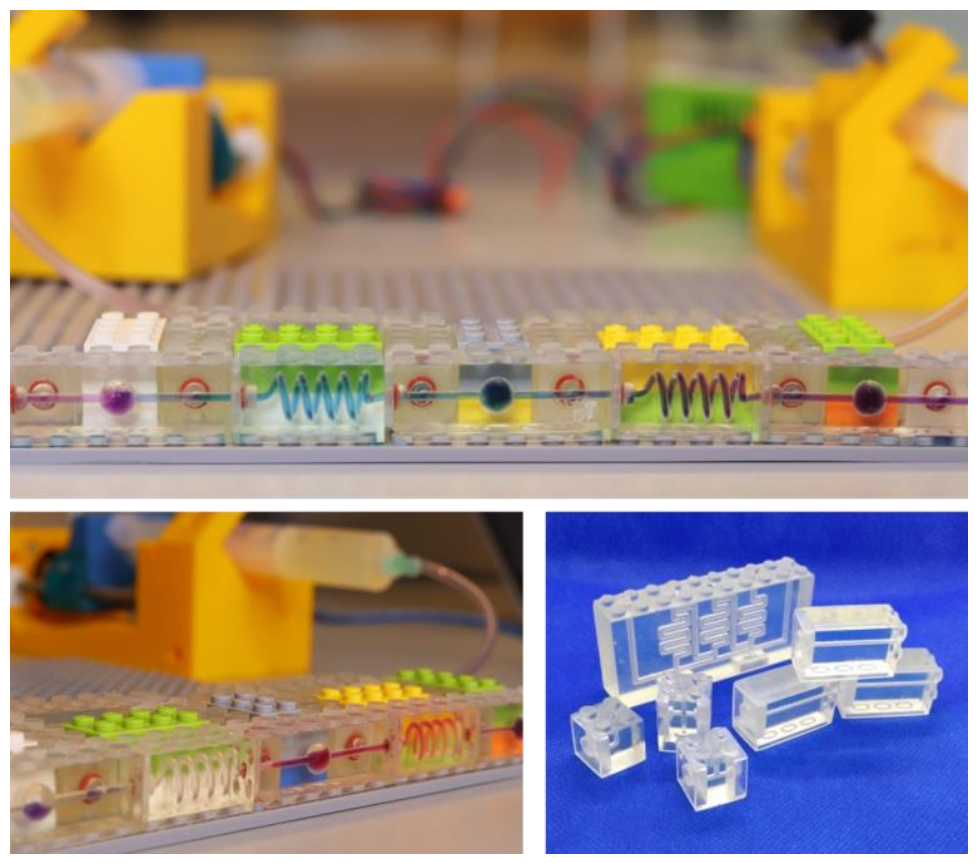

Figure 44- Left and Middle: Assembled Flui.Go pH-Experiment with the Flui.go blocks V.3. Right: an example of printed second generation of Flui.Go blocks.

\subsubsection{Flui.Go as a Company}

All these efforts resulted in the foundation and further development of our spin-off company, Flui.Go Science, which to this day has its own laboratory room and employs 10 trainee students that further develop the microfluidic building blocks and the experiments. Flui.Go has been established with a combined starting capital of 18.000 Euro from the three founders: Renato Rogosic, myself and Univenture, the valorization body of Maastricht University. After achieving our milestones for the first year, further investments of 30.000 Euro have been made by Univenture. 
The unique selling proposition of Flui.Go is the intuitive nature in which our product works. The microfluidic building blocks can be assembled to construct a myriad of different experiments by simply arranging the bricks in different ways. Our kit comes with six different premade experiments, from learning about $\mathrm{pH}$ via color changing cabbage juice, over density experiments by creating layers of colored liquids, to producing 'fruit caviar' via alginate calcification. The students can then add upon those experiments by adding different blocks and see the results change. For pupils and students this adds fun, curiosity and excitement to the learning process and leads to a very organic learning experience which empowers children to experiment and use their creativity.

We developed 10 complete prototype kits, of which an example is shown in Figure 45. The kit contains over 30 bricks, two micropumps, which are also manufactured in house via 3D printing, and the pump-control software. This prototype-set was used to conduct a field-study on its use in primary school education together with Nardi Fanchamps, an assistant professor in educational sciences at the Open Universiteit. The promising data gained from this study is currently prepared in a manuscript for publishing in the near future. Our close collaboration with primary schools for the testing and improvement of our kit also resulted in the first product orders for a total of 52 kits being delivered to 26 schools. 

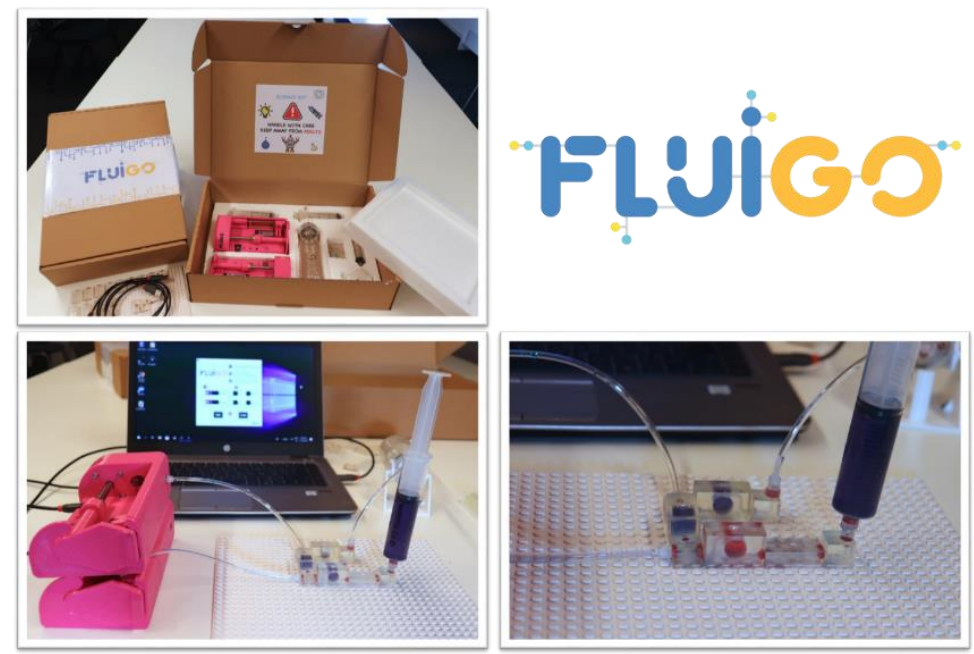

Figure 45 - The advanced product, Pumping system with 2 pumps, syringes and waste container as well as the Flui.Go-bricks, assembled into a version of the pH-experiment

For the future, the market perspectives can be separated into three areas: Schools, private educational companies and the private market. Primary schools already showed great enthusiasm about the product and several schools ordered their first kits. This demonstrates that schools form a very promising market for Flui.Go as there are over 6.000 elementary schools as possible customers in the Netherlands alone [3]. Additionally, Flui.Go can expand to the over 600 secondary schools too [4]. This means that overall about 1.5 million primary and about 1 million secondary school students could benefit from Flui.Go [5]. An expansion into other countries will be supported by the central location the Netherlands has in Europe as well as its highly diverse workforce from different countries, encouraging expansion into different nations. The second potential market, private educational companies like 
Mad Science show an additionally good outlook. Mad Science is a science teaching company that partnered with us from the beginning and supported Flui.Go since the Science + Engineering competition. They are a globally working science education franchise and reach 500.000 children in the Netherlands alone. They also collaborate with $1 / 3$ of primary schools, creating a connected value-network with our first market [6]. They showed interest in Flui.Go to distribute the kits to children after their shows and lectures. While the current pandemic brought the collaboration to a pause until the economy recovers, we developed a Flui.Go collectible system aimed at science educators like Mad Science. Here, our Flui.Go blocks will not be distributed in a big set as shown in Figure 45 but in small increments as 'collectible packs' each having enough parts for one experiment (Figure 46). With this approach, children attending science education events at companies like Mad Science gain a collectible pack. The more events they attend the bigger their collection grows and the more experiments they will be able to perform by putting the blocks from different packs together. This approach helps science education companies to increase event and class attendance and rewards children with fun experiments after each session. With this all parties benefit, increased attendance increases profit for the science education companies and Flui.Go at the same time, and the customer satisfaction increases due to the collectible science experiments that build onto each other. 

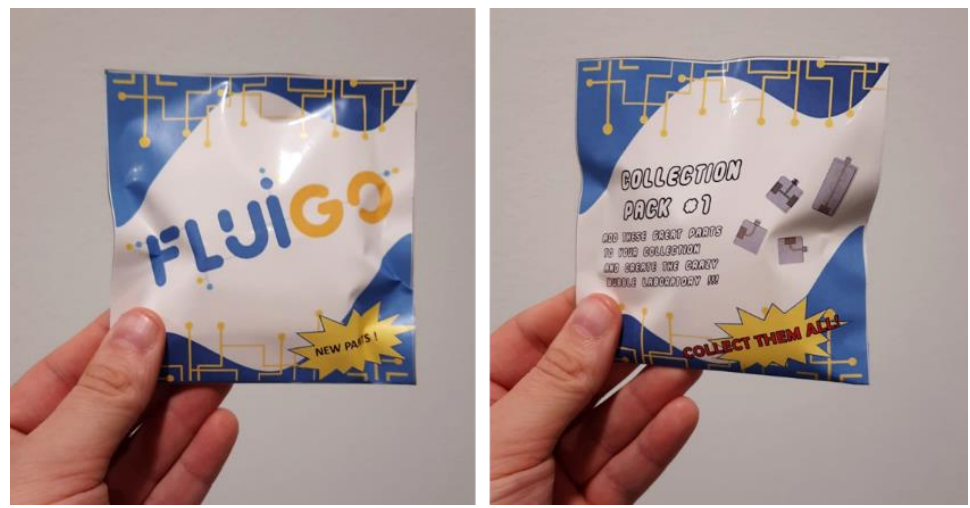

Figure 46 -Mockup of a Flui.Go collection pack, for science education companies.

A third market of interest is the private market. The market revenue for toys in the Netherlands amounts to about 920 million dollar [6]. Making it a highly interesting market for further expansion. The current business strategy is to introduce the product to the market via schools and science education companies in order to gain valuable feedback for our product. After integrating the feedback, we aim to introduce the product into the private market. The present focus is on scaling the production process using the Tovas method, which will be presented in the next chapter. 


\subsection{Transition}

The presented parts are all completely $3 \mathrm{D}$ printed. This has, as was already discussed, a magnitude of benefits, however it also has one large downside: scale-up. For a product like Flui.Go, a scalable production method is beneficial, as the product will eventually not be produced on-demand in LIC, but rather massproduced as a commodity in HIC. For mass production, injection molding is the current method of choice. However, as was discussed in the introduction, this means the channels have to be constructed on the outside. The created microfluidic channels have then to be sealed consecutively. This could be achieved via ultrasonic welding or thermobonding with a second part. But, depending on the geometry, this might make a second mold that produces a matching counterpart necessary, which increases production cost. Therefor a method had to be found that is able to adapt and seal a three dimensional substrate. A method that has the potential to solve this problem can be found in the next chapter. 
3D Printed Microfluidics 


\section{Topographical Vacuum Sealing of 3D printed Multiplanar Microfluidic Structures.}

Adapted from

B. Heidt, R. Rogosic, N. Leoné, E. Brás, T. Cleij, J. Harings, H. Diliën, K. Eersels, B. van Grinsven, Biosensors. 2021 11, 395, DOI: 10.3390/bios11100395.

As well as a pending E.U. Patent under the Application No 21155200.5 . 
Topographical Vacuum Sealing

\subsection{Chapter Context}

Chapter 4 and 5 showed the capabilities and benefits of 3D printed microfluidics and their practical applications. While the benefits of 3D printing are clear, there are also downsides. As presented in chapter 4 , channel construction inside a 3D print is difficult, as channels tend to clog. This is especially the case for complex structures and channels that are not perpendicular to the buildplate as well as for small channel sizes. Open channels that were constructed as grooves on the 3D printed surface however are tremendously easier to produce, but they create another challenge, they need to be sealed. While this is easy if the surface is monoplanar, it becomes challenging for surfaces with complex three-dimensional structures. This is a large problem, given that the ability to create complex threedimensional structures is one of the main benefits of $3 \mathrm{D}$ printing.

To solve this, a completely new method of microfluidic device construction was developed. The method was coined topographical vacuum sealing (Tovas) and it combines a 3D printed substrate containing open surface channels with a lowresolution vacuum forming technique. Here, vacuum-forming is used to align a heated thermoplastic with the 3-dimensional shape of the substrate, in order to follow and seal the surface reliably. The method is low-resolution, meaning the thermoplastic is able to align with the macrostructure but at the same time does not intrude into the microchannel, since they are too small for the thermoplastic to align to. This process is completely novel and a patent application was filed based on this invention. 


\subsection{Introduction}

Microfluidics is a field of growing importance for biosensing, given the enormous capabilities it offers in terms of sample preparation, sample manipulation and reagent storage in minimal form factors that are especially advantageous in pointof-care scenarios. The field of microfluidics started out on the shoulders of micro-electromechanical systems (MEMS) manufacturing, where it applied microchip fabrication technology to create microfluidic channels [1]. MEMS techniques like photolithography remain - despite requiring a clean room and a work-intensive manufacturing process - the gold-standard up until today. Many advances have been made to lift the restrictions of clean-room fabrication [2-4], such as replacement of the vapor-deposited metal-masks with masks that are printed with a conventional laser printer on a transparent surface $[5,6]$. This method enables the user to skip the mask manufacturing with its many in-between steps. Furthermore, some developments forego the use of a mask altogether and use printing technologies to directly print the master mold [7-9]. Other approaches go even further and replace the complete lithographic process with new means of production, for example, the use of shrinkable polymers as a substrate [10,11]. Another approach of cleanroom-free manufacturing is lab-on-a-foil (LoaF) [12]. Here, channels are either cut $[13,14]$ or hot-embossed directly into polymer films [15-17]. This approach also offers the benefit of being compatible with flexible substrates. While most other microfluidic channels are created on a rigid surface, lab-on-afoil systems can bend, though this is still limited by the rigidness of the foil used. Vacuum-forming technology is already utilized in LoaF contexts, but contrary to our novel approach, it is only used in the creation of channels via 
molding, which are then de-molded and sealed in a later step [18-21].

More recently, 3D printing has become increasingly popular for the cleanroom-free fabrication of microfluidic structures, driven by the decrease in price and increase in resolution of available 3D printers within the last 10 years [22]. While their resolution, for the most part, still cannot match classical cleanroom photolithographic techniques, they excel in terms of ease of use and rapid prototyping. Another benefit of 3D printing is the capability to create three-dimensional channels, allowing for new applications and form factors compared to the usually monoplanar traditional methods.

The two most common methods for 3D printing are fused deposit modelling (FDM), where a thermoplastic filament is molten in a heated nozzle and added layer-by-layer to create a three-dimensional construct, and SLA-printing, where a liquid photopolymer is cured by exposition to UV light. The light is either directed by a screen, displaying the whole layer (based on DLP or LCD-screen technology, both summarized as mSLA printing), or by a laser that 'writes' the structure into the polymer (laser SLA printing) [23].

While commercial FDM printers specialized in microfluidics are available and techniques like the ESCARGOT method open up interesting capabilities [24], FDM printers still suffer from a lower resolution compared to SLA printers.

Therefore, SLA printing is often the method of choice to produce small features. For the direct manufacturing of microfluidic channels, it is either possible to create closed channels embedded inside the printed device or as groves on the surface. While the creation of closed channels gives more 
possibilities in terms of three-dimensional creation, it is difficult to produce them in small sizes with common desktop printers, as the smaller the channel size, the more likely it is that resin gets trapped inside and is cured by stray light during printing, resulting in clogging of the channel [25]. Therefore, the creation of surface channels is vastly more reliable and smaller channel sizes can be achieved, but it opens up another problem: the channel needs to be sealed to be of use.

On monoplanar substrates, this is achieved by the use of a sealing plane that is attached to the plane with the open channels by either plasmabonding, thermobonding, ultrasonic welding or simply the use of adhesive tape. This plane creates the outer limit to the open channels and seals them. However, this negates the benefit of $3 \mathrm{D}$ printing to create microfluidic devices in new form factors, as sealing channels this way is challenging and time consuming for difficult topographies that are multi-planar.

In this work, we show an easy and cost-effective way to seal open channels on challenging topographic surfaces using a vacuum-forming approach called topographical vacuum sealing (TOVAS). This technique can be used in combination with 3D-printing (or other nonplanar methods like micromilling), to create complex topographic devices in form factors that were previously impossible. We benchmark the capabilities of the new method in terms of the topography that can be achieved and present three different use cases of the new method.

\subsection{Materials and Methods}

The new approach consists of a multi-planar substrate, which can be 3D printed, injection molded, hot embossed or micromilled. The substrate displays open microfluidic channels on 
its surface. The channels are sealed by using a low-resolution vacuum-forming process that stretches a heated thermoplastic over the multi-planar substrate. The thickness of the thermoplastic ensures that the process remains 'low resolution' and only follows the macro-topography instead of also intruding into the microchannel, as is done by vacuum forming of the channels directly. Figure 47 shows the manufacturing process and Figure 48 shows a benchmarking structure with various challenging geometries that can be covered.

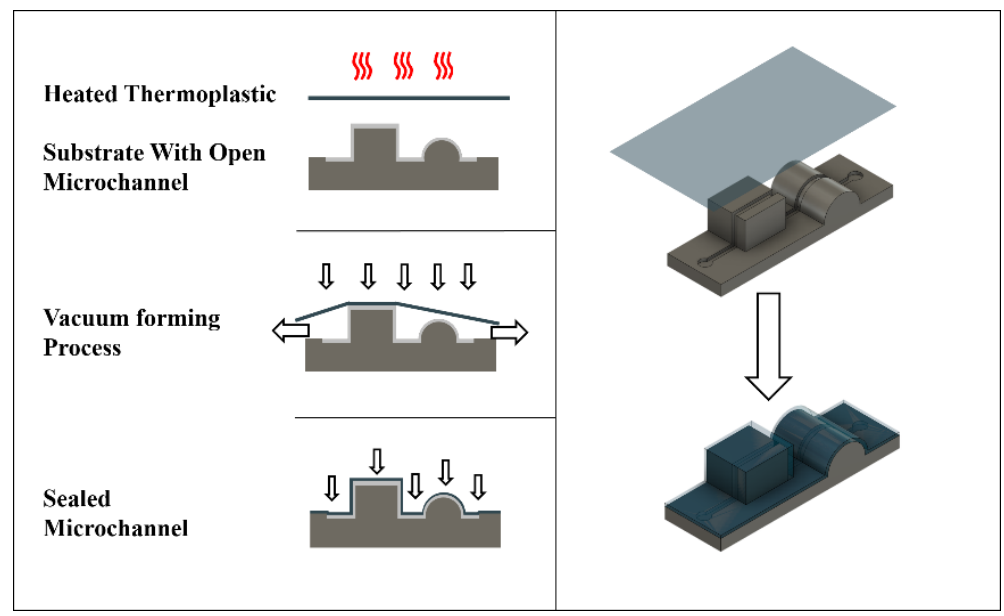

Figure 47 - The fabrication process of topographical vacuum sealing. A thermoplastic is heated above its glass transition temperature and stretched over the topographic substrate. The vacuum aligns the thermoplastic to the surface of the substrate, while the microchannels remain free due to their small size. 


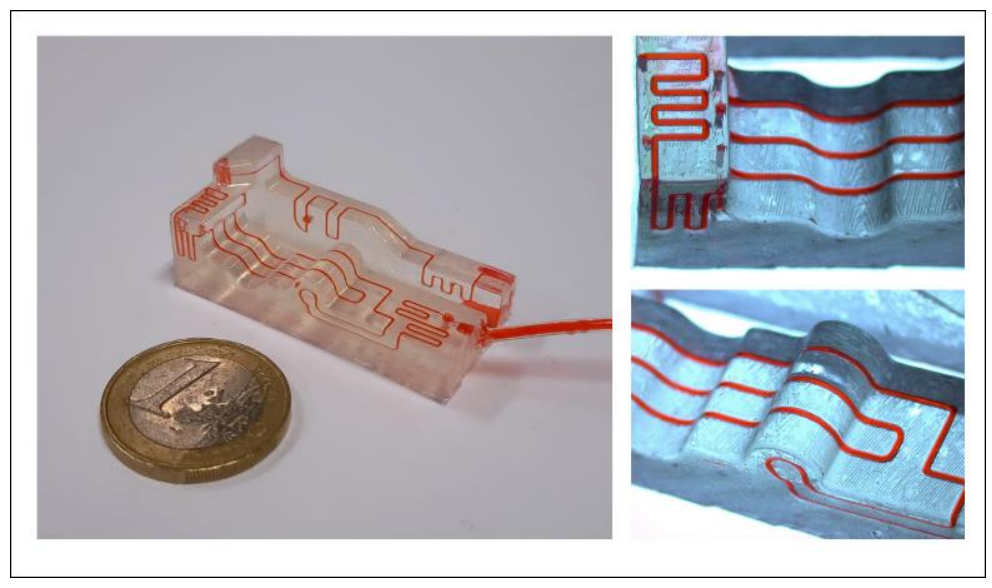

Figure 48 - Testing device covering several challenging geometries and displaying the flexibility of the method..

\subsubsection{Printing and Vacuum Forming}

Several testing structures were designed using Fusion 360 (Version 2.0.9719, Autodesk, San Rafael, CA, USA) and constructed via 3D printing, using a Formlabs Form2 SLA Printer (Formlabs, Somerville, MA, USA). All devices were printed with clear resin V4 (FLGPCL04, Formlabs, Somerville, MA, USA). After printing, the devices were washed for $20 \mathrm{~min}$ in isopropanol (BOOM, Technical Grade, Fischer Scientific, Landsmeer, The Netherlands) in a Form Wash (Formlabs, Somerville, MA, USA) and postcured for at least $20 \mathrm{~min}$ at 60 ${ }^{\circ} \mathrm{C}$ in a Form Cure postcuring machine (Formlabs, Somerville, MA, USA). In devices where geometry makes the resin difficult to wash out, compressed air was used to clean them post-washing, and small holes were flushed with isopropanol to remove the remaining resin. 
After postcuring, a layer of spray adhesive (UHU Spray glueUH46740) was evenly applied over the whole bonding surface of the substrate. A sheet of $500 \mu \mathrm{m}$-thick Polyethylene Terephthalate Glycol (PETG) (Mayku Cast Sheets, Mayku, London, UK) was placed into a vacuum former (JT-018, DIFU Vacuum Former). The machine used is a low-cost device with fixed heating and vacuum settings; it features a movable tray that holds the thermoplastic and is able to be fixed in two positions: In the heating position, the tray is moved upward directly under the heating element, where it is heated. To start the vacuum forming process, the tray is lowered onto the substrate, which is located on a grid above the vacuum pump. The measured variable to ensure repeatability with this type of system is the plastic deformation of the thermoplastic. As the thermoplastic is continuously heated, it surpasses its glass transition temperature and starts to deform; the deformation of the thermoplastic is visible as a clear bulge starting to droop beneath the holding tray. The vacuum-sealing process was started when the thermoplastic reached $1 \mathrm{~cm}$ under the holding tray, which takes $1.5 \mathrm{~min}$ of heating and corresponds to a temperature of $180{ }^{\circ} \mathrm{C}$, as measured with a thermal camera (Akzon HT-18, e = 0.95). The thermoplastic was pulled over the substrate by lowering the tray into the vacuum-forming position and the vacuum pump of the device was activated to introduce a negative pressure measured at -165 mbar for $4 \mathrm{~s}$. This aligned the hot thermoplastic to the substrate surface and thus sealed the prepared topography. An image of the machine and its parts can be found in Supplementary Figure S3. To promote adhesion, the device was left to cure at room temperature and only used after $24 \mathrm{~h}$. Every test device was printed and tested in triplets. 


\subsection{Benchmarking Devices}

\subsubsection{Angle Leakage}

Three types of 3D prints were manufactured to test the channel integrity when traversing different angles. The tested angles were $90^{\circ}, 120^{\circ}, 150^{\circ}, 210^{\circ}, 240^{\circ}, 270^{\circ}$ and $330^{\circ}$. To better visualize leakage, two control channels were fabricated next to the fluid-carrying channel. The fluid channel was flushed with water containing red food coloring. The flushing speed was varied from 1 to $10 \mathrm{~mL} / \mathrm{min}$ in $1 \mathrm{~mL} / \mathrm{min}$ increments. If a channel shows leakage, the control channels will fill and show clear red indicators. The channel size and depth of fluidcarrying and control channels was $500 \mu \mathrm{m}$ and the distance between them $466 \mu \mathrm{m}$. To test if the sealing for $90^{\circ}$ angles could be improved, an additional type of test-structure was prepared with three $90^{\circ}$ angles and different degrees of filleted edges, with $1 \mathrm{~mm}, 1.5 \mathrm{~mm}$ and $2 \mathrm{~mm}$ fillet-radii, respectively.

\subsubsection{Maximum Working Pressure}

To test the maximum pressure which the substratethermoplastic interface can withstand, three test structures were prepared. The structures featured a $500 \mu \mathrm{m}$ dead-end channel flanked by two open-ended $500 \mu \mathrm{m}$ control channels at both sides. The test structures were prepared with different topographies: one monoplanar (flat) structure; one prismshaped topography, with angles of $135^{\circ}$ (base of prism) and $270^{\circ}$ (top of prism); a cube structure with a $90^{\circ}$ angle, containing a $1 \mathrm{~mm}$ fillet (base of cube) and a $270^{\circ}$ angle (top of cube). The structures featured a small reservoir for liquid and a LUER slide adapter to connect the device to a pressure line via a hose. Air pressure was used to force a column of water containing red food coloring out of the small reservoir into the dead-ended channel, increasing the pressure in the process. To 
prevent leakage from the adapter, it was glued in place for every device using PSMT3 Superglue (Pattex, Duesseldorf, Germany).

\subsubsection{Column Separation}

To assess how sensitive the methodology is to the spacing between two large protrusions, test structures were created with varying heights and distances between two adjacent protruding columns. If two columns were too close to each other and/or too high, the thermoplastic would have difficulties covering the space in-between the protrusions. This would limit the form factors of the methodology. Therefore, we created test structures with columns of various heights $(1-6 \mathrm{~mm})$, spaced at different distances from each other $(2-6 \mathrm{~mm})$. The devices were prepared and sealed with the aforementioned method and measured using a digital caliper (DIGI-MET 1320 417, Helios Preisser, Gammertingen, Germany).

\subsubsection{Channel intrusion}

When the thermoplastic is aligned to the substrate macrostructure, it will slightly intrude into the microchannel. To quantify to what extent this occurs, a structure with different channel widths was printed. The thermoplastic was aligned as described but omitting the spray glue to be able to release the formed thermoplastic. The thermoplastic was then used as a mold and filled with Polydimethylsiloxane (PDMS, Sylgard 184) with a ratio of 1:10 curing agent to monomer. After degassing, it was cured for $1.5 \mathrm{~h}$ at $70{ }^{\circ} \mathrm{C}$ to create an imprint of the intrusions. This PDMS imprint was removed from the thermoplastic and cut cross-section. A Leica S6E microscope with a Leica DFC290 HD digital camera was used to take pictures, the prints were aligned to account for the viewing angle and the pictures were quantified using imageJ. 
Translation to PDMS was performed as the thin thermoplastic might have deformed while being cut; this was prevented by using the thicker PDMS imprint that is more resistant to deformation and is easier to observe via a microscopy.

\subsection{Results}

\subsubsection{Angle Leakage}

Figure 49 shows the basic function behind the test structures. The basic test structure consists of a $500 \mu \mathrm{m}$ fluid-carrying channel that is aligned in-between two identical $500 \mu \mathrm{m}$ empty control channels. The adjacent pair of channels serve as visual controls to quickly identify the possibility of a leakage during the liquid injection, as they would be rapidly filled with the colorant. In Figure 49(II-IV) a $270^{\circ}$ crossing without leakage is shown. 
Topographical Vacuum Sealing

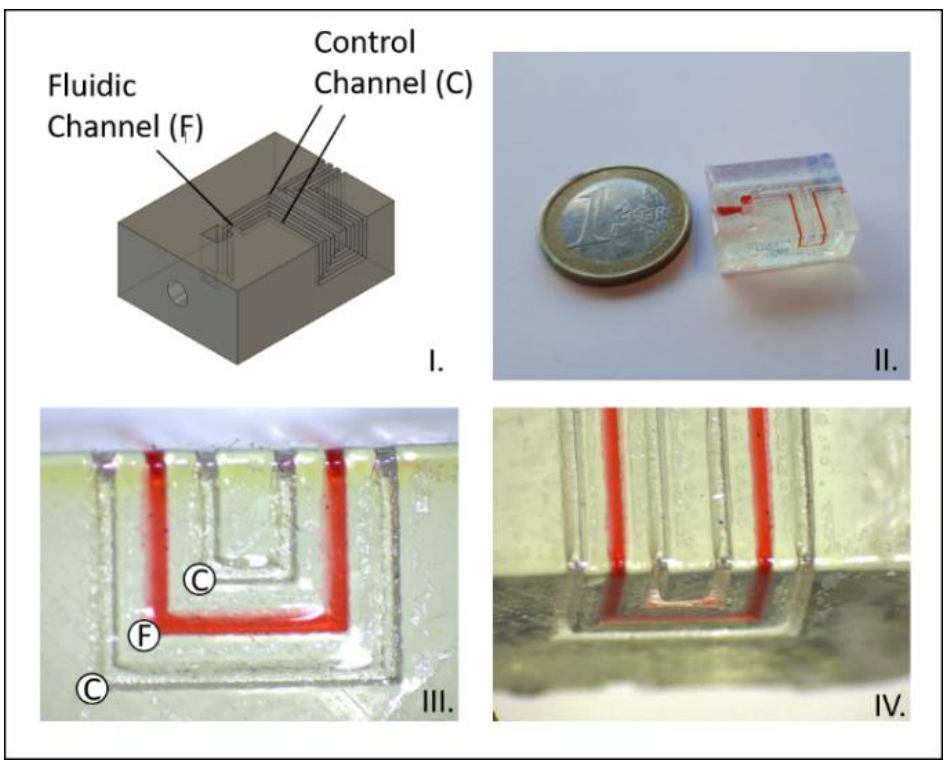

Figure 49 - (I.) Rendering of a basic example of a testing chip with two crossings of a $270^{\circ}$ angle. The control channels are flanking the fluid channel and turn red if they fill due to leakage. (II.) Resulting 3D-print carrying water colored with red food coloring. (III.,IV.) Close-up of the angle crossing with control channels $(C)$ and the fluid carrying channel $(F)$.

The different test structures created to test the methodology are shown in Figure 50. The control channels remained free for all tested flow rates for the channels traversing the angles $330^{\circ}$, $270^{\circ}, 240^{\circ}, 210^{\circ}, 150^{\circ}$ and $120^{\circ}$. Only the $90^{\circ}$ angle leaked, as shown by the filled control channels. All $90^{\circ}$ crossings with filleted edges remained leakage-free, proving that applying a fillet over an angle improves the thermoplastic contact and sealing. 


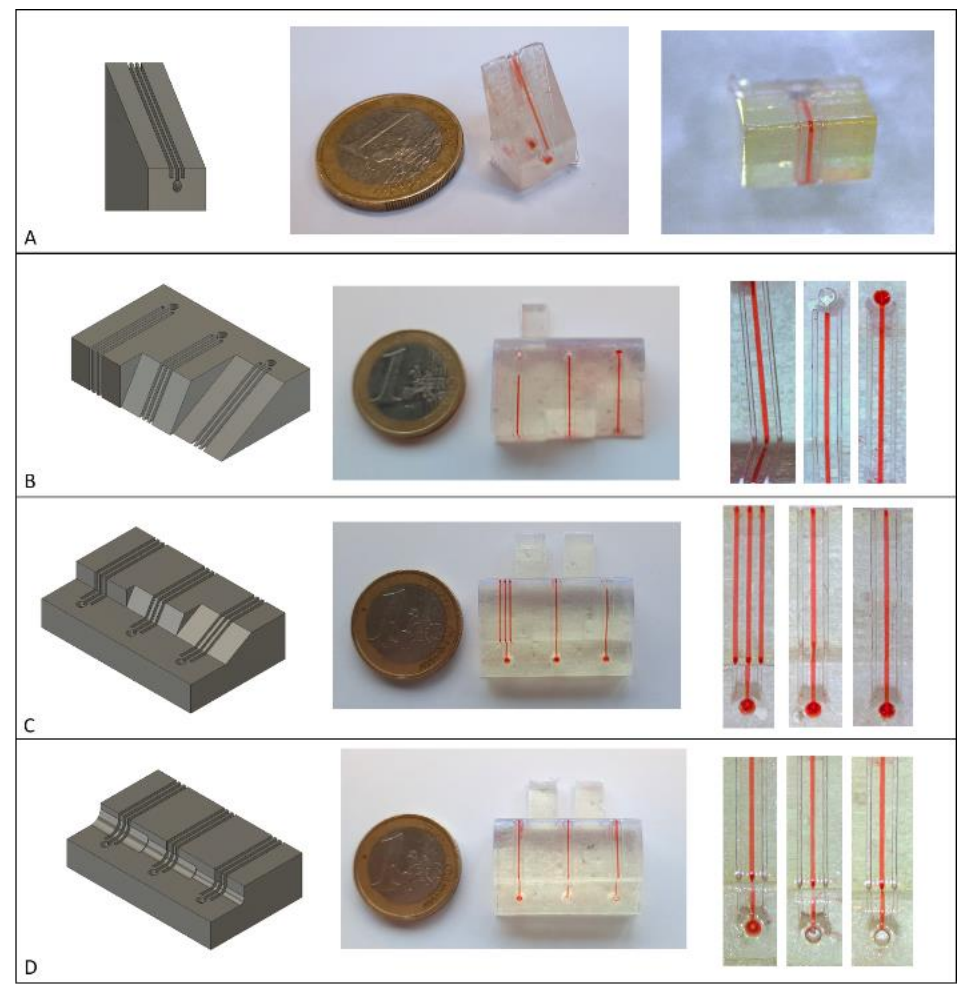

Figure 50 - Different structures for testing channel integrity while traversing different angles. Left: Rendering of the structure. Middle: Completed print sealed with the topographical vacuum forming method. Right: Magnified channels. (A) $330^{\circ}$ angle. (B) From left to right: $270^{\circ} 240^{\circ}$ and $210^{\circ}$ angles. (C) From left to right: $90^{\circ}, 120^{\circ}$ and $150^{\circ}$ angles. (D) From left to right: $90^{\circ}$ angles with $2 \mathrm{~mm}, 1.5 \mathrm{~mm}$ and $1 \mathrm{~mm}$ radius fillets.

\subsubsection{Maximum Pressure}

In the maximum pressure test, a 6-bar pressure was achieved without any leakage in all the created test structures. This was the maximum pressure available in the laboratory. Figure 51 
Topographical Vacuum Sealing

shows the different structures, with the water column forced into the middle channel by the applied pressure.

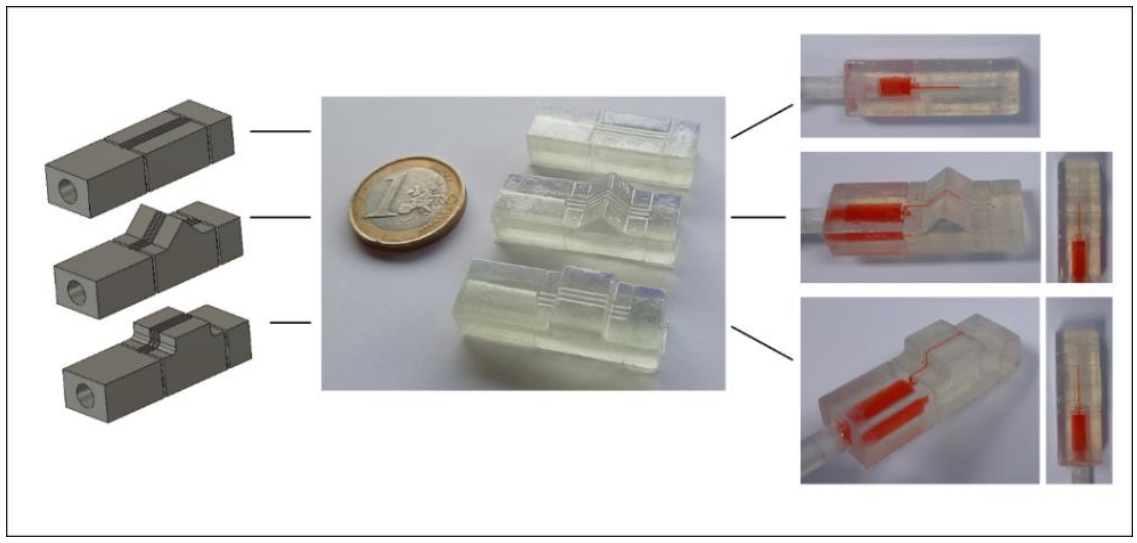

Figure 51 - Different structures for the maximum pressure test. Left: Rendering of the structures. Middle: Completed print, sealed with the topographical vacuum sealing method. Right: Pressurized channel with water. Top line: Monoplanar structure. Middle line: Prism-shaped structure with $135^{\circ}$ and $270^{\circ}$ angles. Bottom line: Cube extrusion with $90^{\circ}$ angle, $1 \mathrm{~mm}$ fillet and $270^{\circ}$ angle.

\subsubsection{Column Separation}

Figure 52 shows the height of the thermoplastic stretched between the columns relative to the bottom of the structure (in other words, how far down the thermoplastic was able to stretch between the two protrusions). The height of the thermoplastic was $0.5 \mathrm{~mm}$, which was chosen as a cut-off value since below this distance, the ground in-between the columns can be considered sealed, which was optically confirmed for every case. The highest hanging thermoplastic is seen in the highest columns that are closest together. For $6 \mathrm{~mm}$ - to $4 \mathrm{~mm}$-high 
columns, no thermoplastic was able to cover the space inbetween. For $3 \mathrm{~mm}$-tall columns, the space in-between was sealed when the columns were 6 and $5 \mathrm{~mm}$ apart. For $2 \mathrm{~mm}$ tall columns, all distances connected to the ground except inbetween the $2 \mathrm{~mm}$-distant columns. Lastly, the $1 \mathrm{~mm}$ height did connect completely even for the columns only $2 \mathrm{~mm}$ apart. In general, the further apart and lower the columns were, the easier it was for the thermoplastic to align with the ground inbetween.

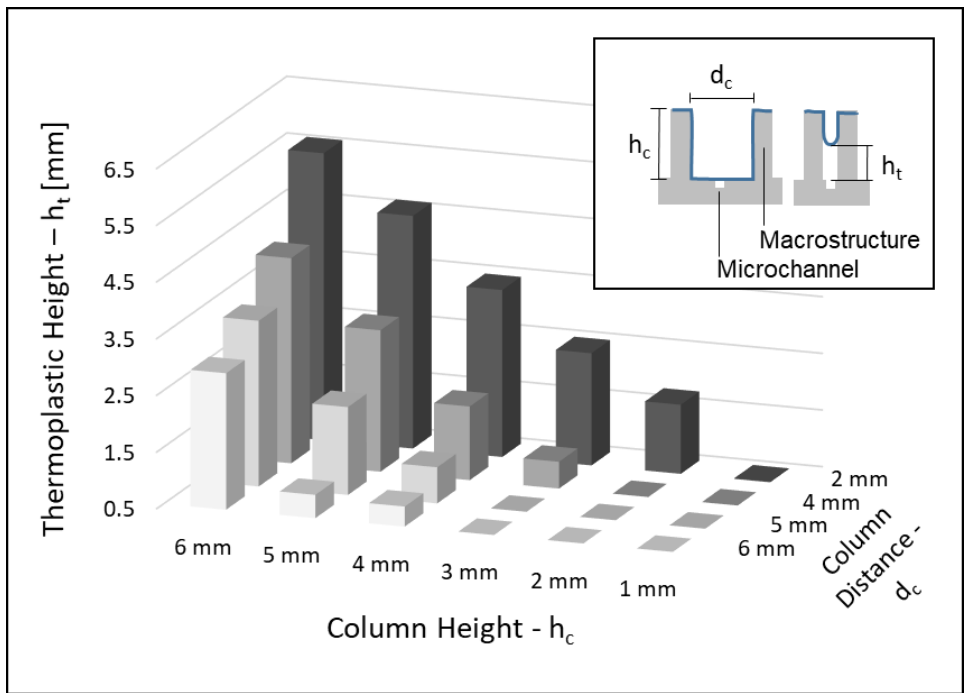

Figure 52 - Relationship between column height, column distance to each other and the height of the thermoplastic spanned in-between the columns. Heights at $0.5 \mathrm{~mm}$ made contact with the substrate, as this is the thickness of the thermoplastic sheets. The complete dataset as a 2D graph is available in the supplementary information. 
Topographical Vacuum Sealing

\subsubsection{Channel Intrusion}

Figure 53 shows the intrusion of thermoplastic into the channels as a percentage of channel width. The $2 \mathrm{~mm}$-wide channels showed the largest intrusion into the channel; with $880 \mu \mathrm{m}$, the intrusion is almost half the channel size $(43.76 \%)$. Channel intrusion decreased with smaller channel sizes. The 1 mm-wide channel only had an intrusion of $200 \mu \mathrm{m}(20.07 \%)$ and the $0.5 \mathrm{~mm}$ wide channel only had an intrusion of $30 \mu \mathrm{m}$ $(5.33 \%)$. The general trend shows that the smaller the microchannel, the less channel intrusion seems to be a problem.

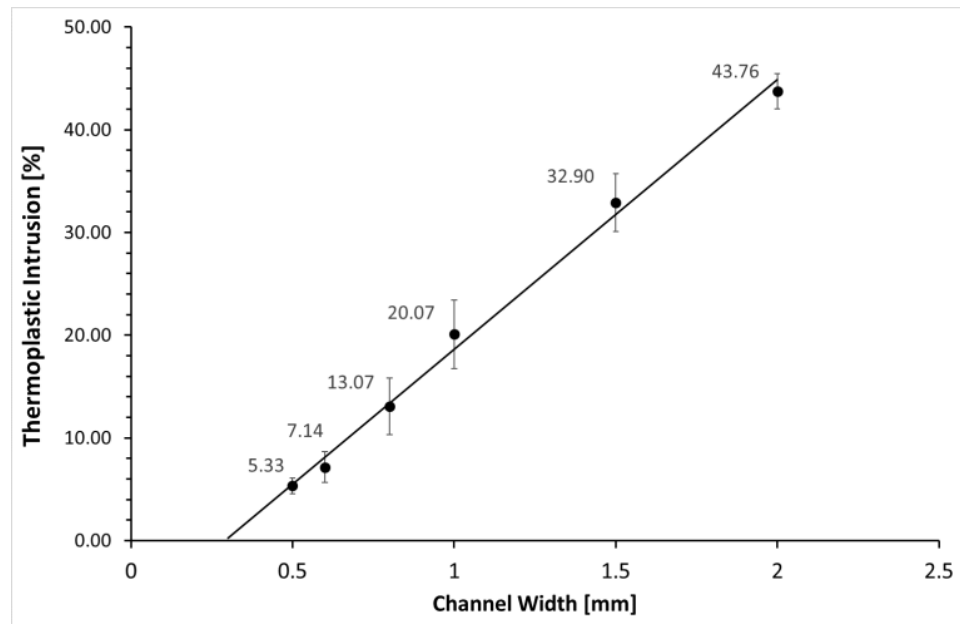

Figure 53 - Thermoplastic intrusion into the channel as percentage of channel width. 


\subsection{Application Examples}

\subsubsection{Application: Roundabout Serpentine Mixer}

The developed methodology allows for the fabrication of a whole new range of applications. In this experiment, we used it to create a serpentine mixer that was formed around a cuboid, with channels wound 12 times around all four sides of the mixer. To illustrate the concept, the mixer was flushed with water containing blue and red food coloring, respectively, at a flow rate of $1 \mathrm{~mL} / \mathrm{min}$ (Figure 54). The methodology enables users to use substrates more efficiently and increase the length of the mixer pathway without changing the volume of the resulting device. It is also possible to extend the concept toward heating-cooling systems by micro-milling similar channels into metal substrates and covering the channels with the same technique, thus drastically increasing the contact area between the liquid and the metal substrate. Here, the glass-transition temperature has to be taken into account $\left(75-80{ }^{\circ} \mathrm{C}\right.$ for PETG) and serves as an upper limit of the application. While this can be too low for applications like chemical synthesis, it is still useful for other use-cases such as, for example, biosensing with the heat-transfer method, where measurements are often conducted at $37^{\circ} \mathrm{C}$ [26-28]. Furthermore, in a similar system, the substrate could be used as electrodes for electrochemical measurements. 
Topographical Vacuum Sealing

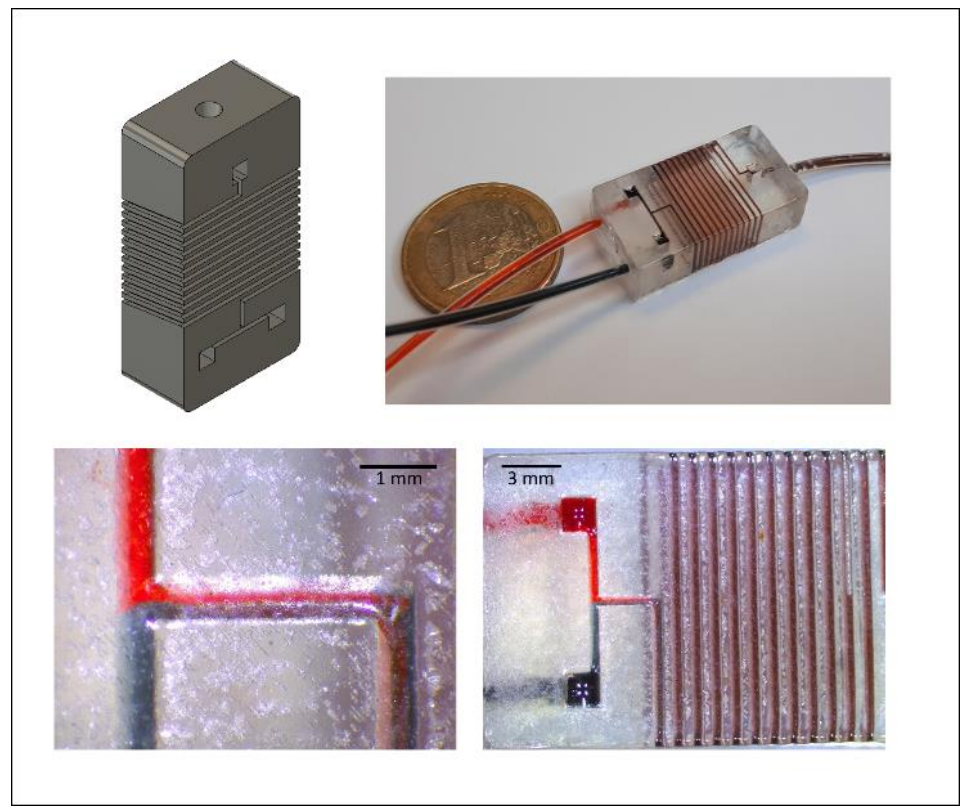

Figure 54 - A meander structure as a serpentine mixer going the whole way around a cube, increasing the usable space.

\subsubsection{Application: UV-Vis Cuvette for Colorimetric Biosensing}

To further illustrate the application potential of the methodology, we developed a device for optical sensing experiments. The device was created with the same width and height as a standard spectrophotometer cuvette, enabling online optical measurement of processes that are compatible with every standard UV/Vis spectrophotometer. The device incorporates molecular-imprinted polymers (MIPs) as synthetic receptors for drug detection. The MIPs were imprinted with the designer drug molecule 2-methoxiphenidine 
(2-MXP). More experimental details on the synthesis procedure can be found in earlier work [29]. After imprinting, the template molecules were washed out and the remaining MIP cavities were loaded with a dye (malachite green). Previous work has demonstrated that when these dye-loaded MIPs come in contact with the target analyte in solution, the loaded dye is displaced by the target molecule and set free in the surrounding solvent, leading to a visibly observable color reaction that can be quantified with a spectrophotometer $[29,30]$. In this experiment, we integrated the MIP-based assay and the cuvette into a singular device, which we used to do a substrate displacement colorimetry (SDC) experiment with a commercial Shimadzu UV-1800 spectrophotometer. The device was loaded with $30 \mathrm{mg}$ of dye-loaded MIP powder, and solutions containing $0.3,0.6$ and $1 \mathrm{mg} / \mathrm{mL}$ MXP were flushed through the Luer adapter into the cuvette (Figure 55). The absorption values obtained from these experiments, performed in triplicate for each experiment, were used to construct a basic dose-response curve, which is shown in Figure 56. These results indicate that the methodology could be used to produce low-cost, user-friendly consumables for SDC (and other biosensor applications) on a large scale. The thermoplastic used is largely optically transparent in the visible range and shows absorption only in the UV range under $350 \mathrm{~nm}$, which makes it highly usable for colorimetric assays. To further account for the optical properties, a blank with di-water was used. 


\section{Topographical Vacuum Sealing}

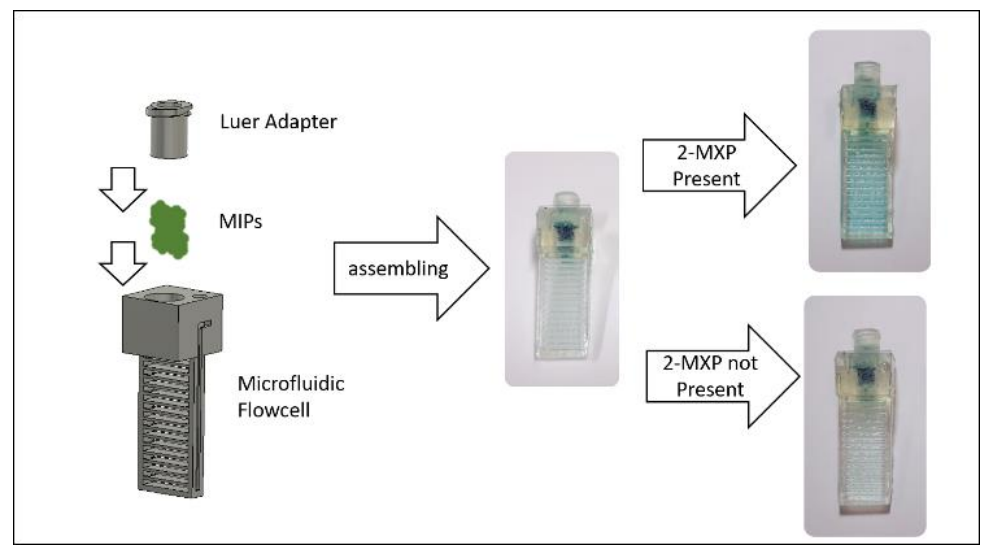

Figure 55 - A microfluidic 'cuvette' with the same width and height as a cuvette; this device can be used in any standard UV/Vis spectrophotometer to conduct online measurements. It is filled with molecular imprinted polymers loaded with a malachite green, which is released on contact with the drug 2-MXP.

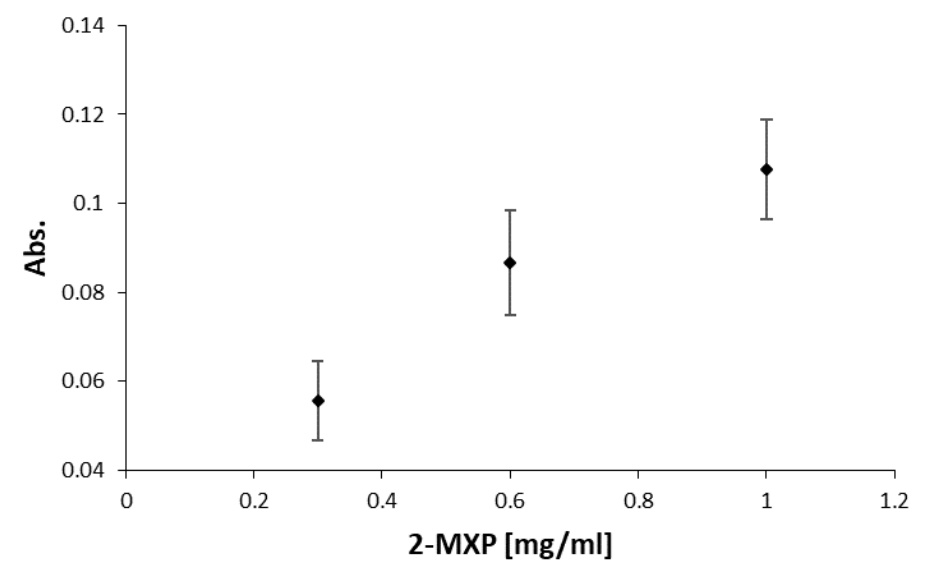

Figure 56 - Absorbance measurement at $617 \mathrm{~nm}$ for the cuvette-shaped microfluidic structure, flushed consecutively with 0.3, 0.6 and $1 \mathrm{mg} / \mathrm{mL} \mathrm{2-}$ MXP solution. 


\subsubsection{Application: Luer-adapter.}

Figure 57 shows a 3D-printed channel with two Luer inlets at both sides. The channel traverses from the bottom of the Luer adapter to the upper side of the channel. Since the critical components are completely on the surface and facing the same direction, the design lends itself to fabrication methods other than 3D printing, for example, such a part can easily be injection-molded since the mold only has to have features at one side. After topographical vacuum sealing, the channels, as well as the Luer-adapter, are closed, isolating the system from the environment. Weakening the thermoplastic above the Luerport, by for example, scratching the surface, enables syringes to easily puncture the system, giving easy access to the Luer port when needed, while isolating it beforehand. The thermoplastic slightly intrudes into the Luer adapter, thus creating a reliable seal at the edge from the internal Lueradapter surface to the top surface of the substrate, something that would be difficult to achieve by sealing with adhesive tape alone. 
Topographical Vacuum Sealing

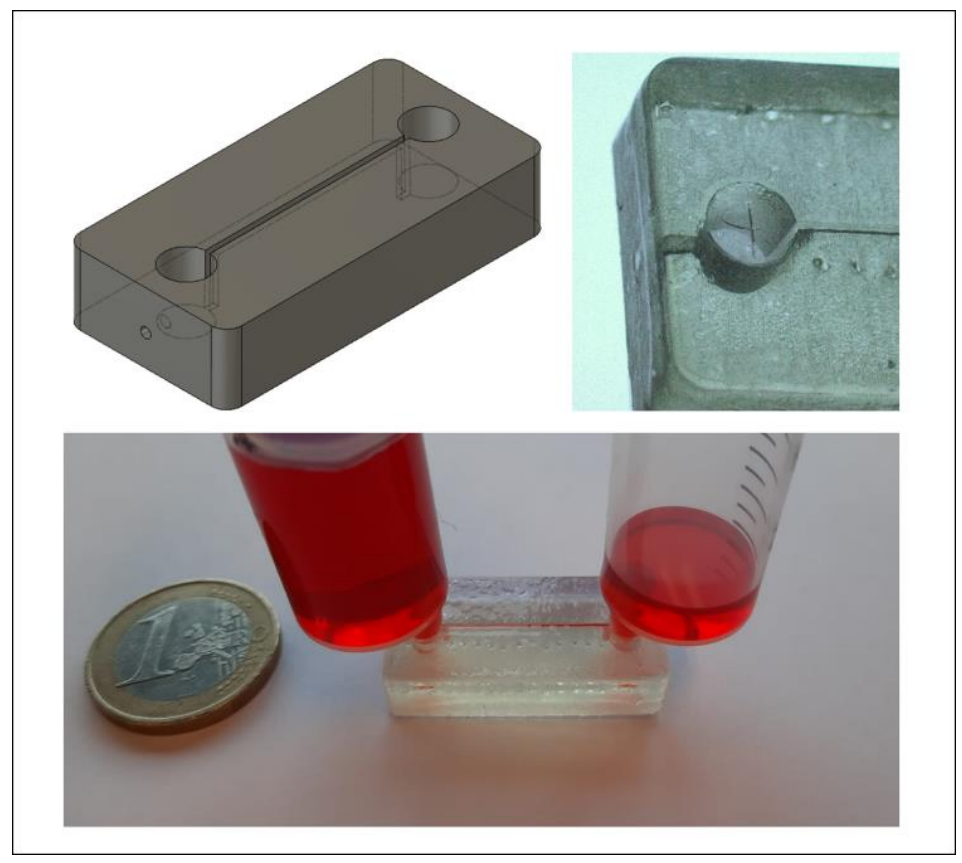

Figure 57 - Channel with 3D-printed Luer-Adapter. The channel traverses completely on the upper surface and thus can be easily injection molded.

\subsection{Conclusions}

We demonstrated a novel method for the creation of topographical microfluidic channels and gave several demonstrations of how the newly possible form factors of the devices can be applied to create microfluidic structures for biosensing applications. The possible applications span a wide range of different fields, for instance, creating devices for healthcare, food safety and even education [31]. 
The method works reliably for a wide array of shapes; the angles covered the range from $90^{\circ}$ to $330^{\circ}$ with only the $90^{\circ}$ angle showing leakage. Applying a fillet to the $90^{\circ}$ angle prevented the leakage, showing that the thermoplastic was most likely unable to intrude and adhere to the tight edge. A rounding of the edge should be possible in almost all designs, eliminating the problem. The column separation experiment showed the relationship between the height and distance of protrusions and the ability of the thermoplastic to align to the full structure. Here, high protrusions in close proximity were more difficult to align to, compared to short protrusions at greater distances from each other. Given that the alignment is dependent on the plasticity of the material, an increase in temperature should improve the alignment of the thermoplastic onto the substrate. A similar improvement might be achieved with an increase in negative pressure or the use of a thermoplastic with a lower glass transition temperature such as polylactic acid. In particular, substrates with difficult geometries, like several protrusions that are high and/or in close proximity to each other, might benefit from these changes. However, increasing the plasticity may also lead to greater unwanted intrusion into the microchannel. Conversely, to minimize the microchannel intrusion, lowering the temperature and vacuum settings, as well as using a thermoplastic with a higher glass transition temperature such as polystyrene or polycarbonate, might achieve better results. This would be especially helpful for substrates that have a simple surface geometry, with low and few protrusions but larger channels, since here, the surface alignment will be less of a problem than the channel intrusion. Therefore, even though the parameters used in this study are widely functional, the process can still be further optimized and adapted to different applications and substrate geometries, to find the best balance between the channel intrusion that was 
shown in Figure 53 and the macro-structure alignment shown in Figure 52, on a case-by-case basis.

The pressure stability of the substrate-thermoplastic interface was measured at up to 6 bar, which is way above that necessitated by most microfluidic applications. This was also the maximum pressure available in the laboratory and thus the actual value is most likely higher. However, the maximum pressure the device can handle is also dependent on the substrate geometry, and while it was tested with different structures, this may be lower in substrates with more challenging features or substrates containing several weak points. Therefore, the measured value is still to be seen as a value under ideal circumstances.

One of the main benefits of the presented method is its ease of use and affordability. A Form3 SLA Printer (the successor of the Form 2 used in this study) can be acquired for 3500 euros, tremendously cheaper than the infrastructure cost of a cleanroom. Furthermore, entry-level mSLA 3D printers are available for around 300 euros; while their quality might not be able keep up with the more expensive machines, they might still be useful for applications with larger dimensions. The vacuum former itself can be acquired for about 100 euros. The complete process can be performed without a cleanroom and with minimal training, as an entry-level vacuum former works with only a lever that moves the heated thermoplastic over the substrate and two buttons to turn the heating and vacuum on and off; the vacuum automatically carries out all the alignment of the thermoplastic. The use of an low-cost vacuum former, of course, has downsides due to its fixed heating and vacuum settings and fewer control points compared to more complex machines, like the ones made for microthermoforming [32]; 
however, the device makes up for it with its lower complexity, ease of use, availability and affordability, which might be especially beneficial for applications in low-income countries, where missing infrastructure can be a large bottleneck to research and the deployment of microfluidic devices, e.g., in point-of-care diagnostics [33]. To add to this, highly expensive devices might simply not be necessary in the first place, given that the aim of the method is to seal the microchannels with a relatively thick layer of thermoplastic. This is less prone to errors compared to creating said microchannels with a much thinner sheet of thermoplastic and higher pressure, such as for use in microthermoforming, which demands more stringent parameter control. Thus, easy to use and cheap devices might have the advantage for this application. Of course, further studies should take a closer look at all given parameters, like the thermoplastic used, the heating and the pressure difference applied (both as negative and positive pressure). However, for this, a new vacuum former needs to be constructed, which was beyond the scope of this study. Here, we have presented a proof-of-concept of the method and showed that the new technology provides an improvement over old monoplanar microfluidics, in terms of price, ease of use and achievable form-factors. Using 3D-printed substrates still results in relatively large channels, due to the limitation of current 3Dprinting technology; however, topographic vacuum forming can also be applied to substrates manufactured by other means such as micro-milling, injection molding or hot embossing. This flexibility also enables this new technique to be used with a multitude of different materials, besides the resin-printed structures that are used. For example, thermoplastic substrates may enable better bonding and can create structures that are completely made from only one material. Metals, on the other hand, may be micro-milled and used as efficient heat 
exchangers or electrodes. Another remaining downside is the adhesion layer that still has to be applied to ensure leakage-free operation. While the use of spray-glue might not be a problem for many experiments, it can present a point of error in others, for example, in cell culture applications. While in this proofof-concept study, a commercial all-round adhesive was used, it may also be possible to use biocompatible adhesives with known properties, applied via airbrush systems. Airbrush application of uncured polyurethane, for example, was carried out by our group for other research and could be used as a bonding layer. Another way in which this might be improved is the use of different thermoplastic sheets, as the thermoplastic we used was designed for molds and was intended to be nonstick. Finetuning of the factors discussed above - heating temperature, vacuums strength and the thermoplastic and substrate material used - might tremendously improve the results and make the use of an adhesive layer redundant.

While there is still room for optimization, this proof-of-concept for topographical vacuum forming already shows the great benefits it can bring to microfluidic manufacturing. In particular, its ease of use, affordability, cleanroom-free fabrication, fast turnaround time and the possibilities for highly advanced form factors make this technology highly attractive to microfluidic researchers, especially given the rising interest in 3D-printed microfluidics. Further developments in 3D printing will go hand in hand with Tovas and increase its area of application since Tovas is compatible with common 3Dprinting techniques $[34,35]$. This is especially interesting since 3D printing is expanding increasingly into areas other than simple structure fabrication. The wide range of materials available for $3 \mathrm{D}$ printing can be used in colorimetric biosensor applications, as demonstrated in this study, but also in other 
potential biosensor applications, for example, in flow cells for fluorescent or electrochemical detection [36-38]. In addition to biosensing, these new 3D-printing materials can also form the basis of smart surfaces that serve additional functions $[39,40]$. Tovas can be a critical component to support this development and make 3D-printed microfluidics easier, cheaper and more available. The low barrier of entry will enable laboratories that are new to microfluidic-manufacturing to construct highly advanced microfluidic devices. Therefore, the presented method is attractive for both old and new microfluidic researchers.

\subsection{Transition}

In this final chapter a major improvement to $3 \mathrm{D}$ printing is made. It overcomes the downsides of $3 \mathrm{D}$ printing discovered in chapter 4, while maintaining its benefits. In context of LIC, one especially important focus is affordability, the price is kept low as vacuum forming machines are available at a low cost. Furthermore, the technique allows to seal complex microfluidic topographies and shapes, this allows an adaption of microfluidic circuits to devices that are already available in LIC. As a result, older devices can be repurposed and new devices do not have to be acquired. This was shown with the example of a microfluidic UV-Vis cuvette that fits into common spectrophotometers. 
Topographical Vacuum Sealing 


\section{Conclusion and Final Thoughts}




\subsection{Aim of the Thesis}

The aim of the thesis was to discuss and improve biosensors from several different angles. The thesis started with an analysis of PoC usage in low-income countries and assesses the bottlenecks they face today, which in turn gives valuable insights for their design in the future. This was followed by a proof-of-application of the heat-transfer-method, a technique promising for PoC use. Two methods were demonstrated for the manufacturing of microfluidics, which can be used for PoC diagnostics as 'lab-on-a-chip' devices. The first was the construction with commonly available 3D printers and the second an improvement upon 3D printing by combining it with low-resolution vacuum forming to create a process called 'topographical vacuum sealing'. Both can be considered enabling technologies for research and manufacturing especially in LIC, due to their innate small investment costs compared to cleanroom-microfluidics. Furthermore, several examples of 3D printed microfluidics were given with a new HTM-flow-cell and the microfluidic building blocks Flui.Go.

The analysis of the current PoC landscape showed a quite complex picture with problems that will not be answered with easy solutions such as 'more funding' or 'cheaper devices'. While funding in research is of course a good start, plenty of problems are found downstream of the value chain. This starts with limited incentives and funding-opportunities for scale-up and manufacturing and IP-considerations. Other social factors that can be problematic for a successful rollout are the proper formation of incentive structures that reward actors along the value chain instead of putting more burden onto them.

An important point for us scientists and (biosensor-)engineers are the questions that involve sensitivity, specificity and 
reliability. Naturally we aim for the very best, but as it has been shown, this might be futile when it leads to greater technical complexity, higher reliance on infrastructure and increased price. For example, a test that has sub-par specificity but is lowcost, widely distributed and has adequate referral structures will outperform a perfectly sensitive and specific test that is too expensive and too reliant on infrastructure to be widely used.

The aspect of infrastructure is an important one, and the benefit of PoC is that samples can be analyzed on the spot without having to rely on sample transport, which often happens per motorcycle in LIC and takes time, decreasing sample quality. In my research concerning the heat-transfer method, it was shown that sample storage at room temperature can have large effects on sample quality and the ability to analyze the samples reliably. Therefore, PoC is immensely important if the cooling chain cannot be guaranteed. The heat-transfer-method has further been shown to be a viable solution for the detection of urinary tract infections and it might be a great solution for LIC due to its affordable sensor setup. For the HTM, the thesis expanded on previous research and showed that the method works even with a large size of urine samples from different genders. Urine is a complex matrix and as such can change tremendously from person to person due to differences in for example water consumption, diet and hormonal differences. While the technology is still in the experimental stage and there is a long way to go for its healthcare implementation, like wide pre-clinical and clinical studies, the shown research already gives a positive outlook and is a further stepping stone on this way.

Another important bottleneck for $\mathrm{PoC}$ is that oftentimes research in HIC follows different design philosophies that 
might not match the environment they are used in later. An example given was that in HIC the most reliable biomarker for a diagnosis is used. This might be a proper choice in HIC, where for example large amounts of blood can be taken, but in LIC a trained phlebotomist might not be available and blood might have to be taken from a pinprick; a smaller sample size in which not enough analyte might be present. Such considerations are often not made in research institutes of HIC. Research conducted in LIC themselves would be beneficial but is often prohibited due to infrastructure constraints in LICs, like missing cleanroom-access. Other bottlenecks can be found in the delivery and stock management of PoC diagnostics. Even when they are available, often stocks run empty and healthcare centers spend month waiting for new material. For both of these points new emerging technologies might be the answer. Here cleanroom-free microfluidic manufacturing using 3D printing might be a first step for on-demand production in small scales as well as giving research-groups in LIC an affordable method for the creation and research of microfluidic channels. The capabilities of 3D printing to manufacture medical parts on demand has already been shown during the COVID-19 pandemic when it was used to, among other things, manufacture respiratory valves. In the future, 3D printing together with other techniques such as on-demand oligo synthesis for example for toehold switches, might offer a valid alternative that helps to conquer problems with supply chain management and stock-outs as PoC devices can be produced decentralized and on-demand.

The benchmarking of the Form2 for microfluidic channel construction made clear that they are somewhat limited in their capability to construct microfluidic channels compared to goldstandard methods such as soft-lithography. While with photo- 
and consecutive soft-litography channels in the micrometer and even nanometer range can be achieved, 3D printing only allows for comparatively large channels of $500 \mu \mathrm{m}$ for embedded and $250 \mu \mathrm{m}$ for surface-channels. However, many biosensor applications do not require small channel sizes, which are used for more complex operations like cell sorting. Therefore, 3D printing with commonly available machines can still be highly beneficial in its use for microfluidic integration of biosensors, especially in LIC.

3D printing has the innate advantage that it can produce complex 3-dimensional shapes, this means new microfluidic structures benefit from new form-factors, which can for example result in a smaller footprint or the adaption to new microfluidic circuits on-top of old readout technologies. However, the big channel size, while still being adequate for most biosensor applications, can be improved to reduce sample size. Compared to embedded channels, surface channels can be produced easier and in smaller sizes. However, they are open on one side and have to be closed in a second step. This is easy with monoplanar substrates, where they could simply be taped shut, however this would negate the benefits of 3D printing which is the complex form-factors that can be achieved. Therefore, the development of topographical vacuum sealing provides an improvement on regular microfluidic 3D printing. Here the channels can still be printed on the surface, and in difficult topographies, as the technique allows the sealing of many form-factors. The method is very affordable as cheap 3D printer and vacuum former can be acquired for under 400 Euro. The microfluidic cuvette that was shown and adapted to a normal UV/VIS-spectrometer illustrate the benefit that $3 \mathrm{D}$ printing can bring to microfluidics, as the microfluidic circuits can easily be adapted to existing devices. This brings additional 
Conclusion and Final Thoughts

benefit for LIC, where existing devices can be used in new ways.

With this, the thesis provides an overview of the current state of PoC diagnostic devices, while highlighting all the bottlenecks faced and suggestions on how to improve upon them. The thesis furthermore brought a promising already existing method, the HTM, into a proof-of-application state and contributed extensive research on 3D printing for microfluidic manufacturing with already established 3D printing methods as well as with the completely novel method topographical vacuum sealing. 


\section{References}

References are given separately for each chapter in order to keep the sequence found in the published articles.

\subsection{References for Chapter 1: Introduction}

[1] The Visual and Data Journalism Team, BBC News.

Covid map: Coronavirus cases, deaths, vaccinations by country. 1-6-2021.

[2] World Health Organisation, WHO Coronavirus (Covid-19) Dashbord. https://covid19.who.int/, accessed: June, 2021.

[3] L. Jones, D. Palumbo, D. Brown, BBC News. Coronavirus: How the pandemic has changed the world economy. 24-1-2021.

[4] V. M. Corman, O. Landt, M. Kaiser, R. Molenkamp, A. Meijer, D. K. Chu, T. Bleicker, S. Brünink, J. Schneider, M. L. Schmidt, D. G. Mulders, B. L. Haagmans, B. van der Veer, S. van den Brink, L. Wijsman, G. Goderski, J.-L. Romette, J. Ellis, M. Zambon, M. Peiris, H. Goossens, C. Reusken, M. P. Koopmans, C. Drosten, Euro surveillance : bulletin Europeen sur les maladies transmissibles $=$ European communicable disease bulletin. 2020 25, DOI: 10.2807/15607917.ES.2020.25.3.2000045.

[5] S. Mervosh, M. Fernandez, The New York Times. Month Into Virus Crisis, U.S. Cities Still Lack Testing Capacity. 6-7-2020.

[6] D. Welle, Germany plans to offer free rapid COVID tests | DW | 16.02.2021, https://www.dw.com/en/germany- 
References

plans-to-offer-free-rapid-covid-tests/a-56587090, accessed: June, 2021.

[7] BBC News. Covid: Tests to be offered twice-weekly to all in England. 5-4-2021.

[8] K. M. Koczula, A. Gallotta, Essays in Biochemistry. 2016 60, 111, DOI: 10.1042/EBC20150012.

[9] C. Figueroa, C. Johnson, N. Ford, A. Sands, S. Dalal, R. Meurant, I. Prat, K. Hatzold, W. Urassa, R. Baggaley, The Lancet HIV. 2018 5, e277-e290, DOI: 10.1016/S23523018(18)30044-4.

[10] J. Cunningham, S. Jones, M. L. Gatton, J. W.

Barnwell, Q. Cheng, P. L. Chiodini, J. Glenn, S. Incardona, C. Kosack, J. Luchavez, D. Menard, S. Nhem, W. Oyibo, R. R. Rees-Channer, I. Gonzalez, D. Bell, Malaria journal. 2019 18, 387, DOI: 10.1186/s12936-019-3028-z.

[11] J.-L. Wu, W.-P. Tseng, C.-H. Lin, T.-F. Lee, M.-Y. Chung, C.-H. Huang, S.-Y. Chen, P.-R. Hsueh, S.-C. Chen, The Journal of infection. 2020 81, 435, DOI:

10.1016/j.jinf.2020.06.023.

[12] S. A. Butler, S. A. Khanlian, L. A. Cole, Clinical Chemistry. 2001 47, 2131, DOI: 10.1093/clinchem/47.12.2131.

[13] Market Data Forecast, Global Pregnancy Testing Market Size, Share, Trends, COVID-19 Impact and Growth Analysis Report - Segmented By Product Type, Test Type, Distribution Channels and Region - Industry Forecast (2020 to 2025). 
[14] W. Villena Gonzales, A. T. Mobashsher, A. Abbosh, Sensors. 2019 19, DOI: 10.3390/s19040800.

[15] R. Rajendran, G. Rayman, Journal of Diabetes Science and Technology. 2014 8, 1081, DOI: $10.1177 / 1932296814538940$.

[16] N. H. Cho, J. E. Shaw, S. Karuranga, Y. Huang, J. D. Da Rocha Fernandes, A. W. Ohlrogge, B. Malanda, Diabetes Research and Clinical Practice. 2018 138, 271, DOI:

10.1016/j.diabres.2018.02.023.

[17] M. D. Hughes, Journal of Diabetes Science and Technology. 2009 3, 1219, DOI: $10.1177 / 193229680900300530$.

[18] F.-G. Banica, Chemical sensors and biosensors, John Wiley \& Sons Inc, Chichester, West Sussex, U.K. 2012.

[19] I. E. Tothill, A. Turner, in Encyclopedia of food sciences and nutrition Academic Press, Amsterdam 2003, pp. 489-499.

[20] M. Sonker, V. Sahore, A. T. Woolley, Analytica Chimica Acta. 2017 986, 1, DOI: 10.1016/j.aca.2017.07.043.

[21] M. A. Morales, J. M. Halpern, Bioconjugate

Chemistry. 2018 29, 3231, DOI:

10.1021/acs.bioconjchem.8b00592.

[22] G. Köhler, C. Milstein, Nature. 1975 256, 495, DOI: $10.1038 / 256495 \mathrm{a} 0$.

[23] P. Holzlöhner, K. Hanack, Journal of visualized experiments : JoVE, DOI: 10.3791/54832. 
References

[24] J. Z. Moraes, B. Hamaguchi, C. Braggion, E. R. Speciale, F. B. V. Cesar, G. d. F. S. Da Soares, J. H. Osaki, T. M. Pereira, R. B. Aguiar, Current Research in Immunology. 2021 2, 32, DOI: 10.1016/j.crimmu.2021.03.002.

[25] H. A. Parray, S. Shukla, S. Samal, T. Shrivastava, S. Ahmed, C. Sharma, R. Kumar, International immunopharmacology. 2020 85, 106639, DOI:

10.1016/j.intimp.2020.106639.

[26] R. V. Kholodenko, D. V. Kalinovsky, I. I. Doronin, E. D. Ponomarev, I. V. Kholodenko, Current Medicinal Chemistry. 2019 26, 396, DOI: $10.2174 / 0929867324666170817152554$.

[27] K. T. Xenaki, S. Oliveira, P. M. P. van Bergen En Henegouwen, Frontiers in immunology. 2017 8, 1287, DOI: 10.3389/fimmu.2017.01287.

[28] J. McCafferty, A. D. Griffiths, G. Winter, D. J. Chiswell, Nature. 1990 348, 552, DOI: 10.1038/348552a0.

[29] B. van Dorst, J. Mehta, E. Rouah-Martin, R. Blust, J. Robbens, Methods (San Diego, Calif.). 2012 58, 56, DOI: 10.1016/j.ymeth.2012.07.011.

[30] G. P. Smith, Science. 1985 228, 1315, DOI: 10.1126/science.4001944.

[31] L. Ledsgaard, M. Kilstrup, A. Karatt-Vellatt, J. McCafferty, A. H. Laustsen, Toxins. 2018 10, DOI: 10.3390/toxins 10060236 .

[32] H. H. Nguyen, S. H. Lee, U. J. Lee, C. D. Fermin, M. Kim, Materials. 2019 12, DOI: 10.3390/ma12010121. 
[33] D. Berdat, A. C. Martin Rodríguez, F. Herrera, M. A. M. Gijs, Lab Chip. 2008 8, 302, DOI: 10.1039/b712609c.

[34] M. H. Shamsi, H.-B. Kraatz, The Analyst. 2011 136, 3107, DOI: 10.1039/C1AN15253J.

[35] N. Vanden Bon, B. van Grinsven, M. S. Murib, W. S. Yeap, K. Haenen, W. de Ceuninck, P. Wagner, M. Ameloot, $\mathrm{V}$. Vermeeren, L. Michiels, International Journal of Nanomedicine. 2014 9, 1629, DOI: 10.2147/IJN.S58692.

[36] A. A. Green, P. A. Silver, J. J. Collins, P. Yin, Cell. 2014 159, 925, DOI: 10.1016/j.cell.2014.10.002.

[37] W. Zhou, P.-J. J. Huang, J. Ding, J. Liu, The Analyst. 2014 139, 2627, DOI: 10.1039/c4an00132j.

[38] Z. Zhuo, Y. Yu, M. Wang, J. Li, Z. Zhang, J. Liu, X. $\mathrm{Wu}, \mathrm{A}$. Lu, G. Zhang, B. Zhang, International journal of molecular sciences. 2017 18, DOI: 10.3390/ijms18102142.

[39] C. Tuerk, L. Gold, Science (New York, N.Y.). 1990 249, 505, DOI: 10.1126/science.2200121.

[40] V. Crivianu-Gaita, M. Thompson, Biosensors \& bioelectronics. 2016 85, 32, DOI: 10.1016/j.bios.2016.04.091.

[41] A. Du Plessis, C. Broeckhoven, I. Yadroitsava, I. Yadroitsev, C. H. Hands, R. Kunju, D. Bhate, Additive Manufacturing. 2019 27, 408, DOI:

10.1016/j.addma.2019.03.033.

[42] F. Cui, Z. Zhou, H. S. Zhou, Sensors (Basel, Switzerland). 2020 20, DOI: 10.3390/s20040996.

[43] K. Eersels, P. Lieberzeit, P. Wagner, ACS Sens. 2016 1, 1171, DOI: 10.1021/acssensors.6b00572. 
References

[44] M. Cieplak, W. Kutner, Trends in biotechnology. 2016 34, 922, DOI: 10.1016/j.tibtech.2016.05.011.

[45] M. Gao, Y. Gao, G. Chen, X. Huang, X. Xu, J. Lv, J. Wang, D. Xu, G. Liu, Frontiers in Chemistry. 2020 8, 616326, DOI: 10.3389/fchem.2020.616326.

[46] J. W. Lowdon, H. Diliën, P. Singla, M. Peeters, T. J. Cleij, B. van Grinsven, K. Eersels, Sensors and Actuators B: Chemical. 2020 325, 128973, DOI:

10.1016/j.snb.2020.128973.

[47] J. Wackerlig, R. Schirhagl, Anal. Chem. 2016 88, 250, DOI: 10.1021/acs.analchem.5b03804.

[48] M. E. Brown, D. A. Puleo, Chemical engineering journal (Lausanne, Switzerland : 1996). 2008 137, 97, DOI: 10.1016/j.cej.2007.09.002.

[49] C. Dong, H. Shi, Y. Han, Y. Yang, R. Wang, J. Men, European Polymer Journal. 2021 145, 110231, DOI:

10.1016/j.eurpolymj.2020.110231.

[50] Y. Wang, Z. Zhang, V. Jain, J. Yi, S. Mueller, J. Sokolov, Z. Liu, K. Levon, B. Rigas, M. H. Rafailovich, Sensors and Actuators B: Chemical. 2010 146, 381, DOI: 10.1016/j.snb.2010.02.032.

[51] S. Tokonami, Y. Nakadoi, M. Takahashi, M. Ikemizu, T. Kadoma, K. Saimatsu, Q. Le Dung, H. Shiigi, T. Nagaoka, Analytical chemistry. $\mathbf{2 0 1 3} 85$, 4925, DOI:

10.1021/ac3034618.

[52] S. Tokonami, K. Saimatsu, Y. Nakadoi, M. Furuta, H. Shiigi, T. Nagaoka, Analytical sciences : the international 
journal of the Japan Society for Analytical Chemistry. 2012 28, 319, DOI: 10.2116/analsci.28.319.

[53] S. M. DePorter, I. Lui, B. R. McNaughton, Soft

Matter. 2012 8, 10403, DOI: 10.1039/c2sm25622c.

[54] T. Shiomi, M. Matsui, F. Mizukami, K. Sakaguchi, Biomaterials. 2005 26, 5564, DOI:

10.1016/j.biomaterials.2005.02.007.

[55] A. Cumbo, B. Lorber, P. F.-X. Corvini, W. Meier, P. Shahgaldian, Nature communications. 2013 4, 1503, DOI:

$10.1038 /$ ncomms 2529 .

[56] O. Hayden, F. L. Dickert, Adv. Mater. 2001 13, 1480, DOI: 10.1002/1521-4095(200110)13:19<1480::AIDADMA1480>3.0.CO;2-V.

[57] O. Hayden, P. A. Lieberzeit, D. Blaas, F. L. Dickert, Adv. Funct. Mater. 2006 16, 1269, DOI:

10.1002/adfm.200500626.

[58] H.-Y. Lin, C.-Y. Hsu, J. L. Thomas, S.-E. Wang, H.C. Chen, T.-C. Chou, Biosensors and Bioelectronics. 2006 22, 534, DOI: 10.1016/j.bios.2006.07.038.

[59] N. W. Turner, C. W. Jeans, K. R. Brain, C. J. Allender, V. Hlady, D. W. Britt, Biotechnology Progress. 2006 22, 1474, DOI: 10.1021/bp060122g.

[60] G. M. Birnbaumer, P. A. Lieberzeit, L. Richter, R. Schirhagl, M. Milnera, F. L. Dickert, A. Bailey, P. Ertl, Lab Chip. 2009 9, 3549, DOI: 10.1039/b914738a.

[61] O. Hayden, R. Bindeus, F. L. Dickert, Meas. Sci.

Technol. 2003 14, 1876, DOI: 10.1088/0957-0233/14/11/004. 
References

[62] R. Arreguin-Campos, K. L. Jiménez-Monroy, H. Diliën, T. J. Cleij, B. van Grinsven, K. Eersels, Biosensors. 2021 11, DOI: 10.3390/bios11020046.

[63] E. Steen Redeker, K. Eersels, O. Akkermans, J. Royakkers, S. Dyson, K. Nurekeyeva, B. Ferrando, P. Cornelis, M. Peeters, P. Wagner, H. Diliën, B. van Grinsven, T. J. Cleij, ACS infectious diseases. 2017 3, 388, DOI: 10.1021/acsinfecdis.7b00037.

[64] O. Hayden, K.-J. Mann, S. Krassnig, F. L. Dickert, Angewandte Chemie (International ed. in English). 2006 45, 2626, DOI: 10.1002/anie.200502857.

[65] K. Eersels, B. van Grinsven, A. Ethirajan, S. Timmermans, K. L. Jiménez Monroy, J. F. J. Bogie, S. Punniyakoti, T. Vandenryt, J. J. A. Hendriks, T. J. Cleij, Daemen, Mat J. A. P., V. Somers, W. de Ceuninck, P. Wagner, ACS Applied Materials \& Interfaces. 2013 5, 7258, DOI: 10.1021/am401605d.

[66] A. Mujahid, F. L. Dickert, Sensors. 2015 16, DOI: 10.3390/s16010051.

[67] S. Piletsky, F. Canfarotta, A. Poma, A. M. Bossi, S. Piletsky, Trends in biotechnology. 2020 38, 368, DOI: 10.1016/j.tibtech.2019.10.002.

[68] Z. Fu, Y.-C. Lu, J. J. Lai, Chonnam medical journal. 2019 55, 86, DOI: 10.4068/cmj.2019.55.2.86.

[69] M. Pohanka, Sensors. 2015 15, 13752, DOI: 10.3390/s150613752. 
[70] L. Yu, Z. Shi, C. Fang, Y. Zhang, Y. Liu, C. Li, Biosensors \& bioelectronics. 2015 69, 307, DOI:

10.1016/j.bios.2015.02.035.

[71] D. Gasperino, T. Baughman, H. V. Hsieh, D. Bell, B. H. Weigl, Annual Review of Analytical Chemistry. 2018 11, 219, DOI: 10.1146/annurev-anchem-061417-125737.

[72] L. Anfossi, F. Di Nardo, S. Cavalera, C. Giovannoli, C. Baggiani, Biosensors. 2018 9, 2, DOI: 10.3390/bios9010002.

[73] Y. Gao, X. Huang, Y. Zhu, Z. Lv, Journal of Immunoassay and Immunochemistry. 2018 39, 351, DOI: 10.1080/15321819.2018.1515775.

[74] A. E. Urusov, A. V. Zherdev, B. B. Dzantiev, Biosensors. 2019 9, 89, DOI: 10.3390/bios9030089.

[75] H. V. Hsieh, J. L. Dantzler, B. H. Weigl, Diagnostics. 2017 7, 29, DOI: 10.3390/diagnostics7020029.

[76] A. K. Yetisen, M. S. Akram, C. R. Lowe, Lab on a Chip. 2013 13, 2210, DOI: 10.1039/C3LC50169H.

[77] R. Flores, Analytical Biochemistry. 1978 88, 605, DOI: 10.1016/0003-2697(78)90462-1.

[78] P. Pokhrel, S. Jha, B. Giri, Practical laboratory medicine. 2020 21, e00166, DOI:

10.1016/j.plabm.2020.e00166.

[79] M. Pohanka, Reviews in Analytical Chemistry. 2020 39, 20, DOI: 10.1515/revac-2020-0111.

[80] J. W. Lowdon, K. Eersels, R. Rogosic, B. Heidt, H. Diliën, E. S. Redeker, M. Peeters, B. van Grinsven, T. J. Cleij, 
References

Sensors and Actuators B: Chemical. 2019 282, 137, DOI: 10.1016/j.snb.2018.11.053.

[81] J. W. Lowdon, K. Eersels, R. Arreguin-Campos, M. Caldara, B. Heidt, R. Rogosic, K. L. Jimenez-Monroy, T. J. Cleij, H. Diliën, B. van Grinsven, Molecules (Basel, Switzerland). 2020 25, DOI: 10.3390/molecules25225222.

[82] R. Karoui, C. Blecker, Food Bioprocess Technol. 2011 4, 364, DOI: 10.1007/s11947-010-0370-0.

[83] S.-W. Kim, J. Roh, C.-S. Park, Journal of pathology and translational medicine. 2016 50, 411, DOI: 10.4132/jptm.2016.08.08.

[84] F. S. Ligler, in Biophotonics Springer, Berlin 2008, pp. 199-215.

[85] F. Huang, F. Xia, in Biosensors based on sandwich assays Springer, Singapore 2018, pp. 29-45.

[86] H. D. VanGuilder, K. E. Vrana, W. M. Freeman, BioTechniques. 2008 44, 619, DOI: 10.2144/000112776.

[87] B. Juskowiak, Analytical and bioanalytical chemistry. 2011 399, 3157, DOI: 10.1007/s00216-010-4304-5.

[88] V. V. Didenko, BioTechniques. 2001 31, 1106-16, 1118, 1120-1, DOI: 10.2144/01315rv02.

[89] R. M. Clegg, in DNA structures, Vol. 211 Elsevier 1992, pp. 353-388.

[90] T. Forster, Naturwissenschaften. 1946 33, 166, DOI: 10.1007/BF00585226. 
[91] E. Reynisson, M. H. Josefsen, M. Krause, J. Hoorfar, Journal of Microbiological Methods. 2006 66, 206, DOI: 10.1016/j.mimet.2005.11.006.

[92] S. Tyagi, F. R. Kramer, Nature Biotechnology. 1996 14, 303, DOI: 10.1038/nbt0396-303.

[93] P. Zhang, T. Beck, W. Tan, Angewandte Chemie International Edition. 2001 40, 402, DOI: 10.1002/15213773(20010119)40:2<402::AID-ANIE402>3.0.CO;2-I.

[94] T. H. T. Chau, D. H. A. Mai, D. N. Pham, H. T. Q. Le, E. Y. Lee, International journal of molecular sciences. 2020 21, DOI: 10.3390/ijms21093192.

[95] S. Findeiß, M. Etzel, S. Will, M. Mörl, P. F. Stadler, Sensors. 2017 17, DOI: 10.3390/s17091990.

[96] M. Nirschl, F. Reuter, J. Vörös, Biosensors. 2011 l, 70, DOI: 10.3390/bios 1030070.

[97] X. D. Hoa, A. G. Kirk, M. Tabrizian, Biosensors and Bioelectronics. 2007 23, 151, DOI:

10.1016/j.bios.2007.07.001.

[98] A. B. Dahlin, S. Chen, M. P. Jonsson, L. Gunnarsson, M. Käll, F. Höök, Anal. Chem. 2009 81, 6572, DOI:

10.1021/ac901175k.

[99] C. Larsson, M. Rodahl, F. Höök, Anal. Chem. 2003 75, 5080, DOI: 10.1021/ac034269n.

[100] R. Funari, K.-Y. Chu, A. Q. Shen, Biosensors \& bioelectronics. 2020 169, 112578, DOI:

10.1016/j.bios.2020.112578. 
References

[101] A. Garifullina, A. Q. Shen, Anal. Chem. 2019 91, 15090, DOI: 10.1021/acs.analchem.9b03780.

[102] N. Bhalla, S. Sathish, C. J. Galvin, R. A. Campbell, A. Sinha, A. Q. Shen, ACS Applied Materials \& Interfaces. 2018 10, 219, DOI: 10.1021/acsami.7b15396.

[103] K. A. Willets, R. P. van Duyne, Annual Review of Physical Chemistry. 2007 58, 267, DOI:

10.1146/annurev.physchem.58.032806.104607.

[104] M. E. Stewart, C. R. Anderton, L. B. Thompson, J. Maria, S. K. Gray, J. A. Rogers, R. G. Nuzzo, Chemical Reviews. 2008 108, 494, DOI: 10.1021/cr068126n.

[105] L. L. Sun, Y. S. Leo, X. Zhou, W. Ng, T. I. Wong, J. Deng, Materials science for energy technologies. 2020 3, 274, DOI: 10.1016/j.mset.2019.10.007.

[106] D. Grieshaber, R. MacKenzie, J. Vörös, E. Reimhult, Sensors. 2008 8, 1400, DOI: 10.3390/s80314000.

[107] Dalvi. 2013.

[108] A. Chaubey, B. D. Malhotra, Biosensors and Bioelectronics. 2002 17, 441, DOI: 10.1016/s09565663(01)00313-x.

[109] P. B. Luppa, L. J. Sokoll, D. W. Chan, Clinica Chimica Acta. 2001 314, 1, DOI: 10.1016/s00098981(01)00629-5.

[110] A. Rebel, M. A. Rice, B. G. Fahy, Journal of Diabetes Science and Technology. 2012 6, 396, DOI: 10.1177/193229681200600228. 
[111] H. G. Wahl, Best Practice \& Research Clinical Anaesthesiology. 2009 23, 387, DOI:

10.1016/j.bpa.2009.09.003.

[112] S. Ferri, K. Kojima, K. Sode, Journal of Diabetes Science and Technology. 2011 5, 1068, DOI:

10.1177/193229681100500507.

[113] M. H. Fahmy Taha, H. Ashraf, W. Caesarendra, ASI. 2020 3, 32, DOI: 10.3390/asi3030032.

[114] Y. Fu, R. Yuan, L. Xu, Y. Chai, Y. Liu, D. Tang, Y. Zhang, Journal of Biochemical and Biophysical Methods.

2005 62, 163, DOI: 10.1016/j.jbbm.2004.11.003.

[115] M. J. Schöning, A. Poghossian, Electroanalysis. 2006 18, 1893, DOI: 10.1002/elan.200603609.

[116] K. K. Kanazawa, J. G. Gordon, Anal. Chem. 1985 57, 1770, DOI: $10.1021 / \mathrm{ac00285a062.}$

[117] M. A. Cooper, V. T. Singleton, Journal of molecular recognition : JMR. 2007 20, 154, DOI: 10.1002/jmr.826.

[118] N. A. Saad, S. K. Zaaba, A. Zakaria, L. M.

Kamarudin, K. Wan, A. B. Shariman, in: Proceedings $20142^{\text {nd }}$ International Conference on Electronic Design (ICED), Penang, Malaysia, 8/19/2014 - 8/21/2014, in $20142^{\text {nd }}$

International Conference on Electronic Design (ICED) (IEEE), pp. 455-460.

[119] R. Weigel, D. P. Morgan, J. M. Owens, A. Ballato, K. M. Lakin, K. Hashimoto, C. Ruppel, IEEE Trans. Microwave Theory Techn. 2002 50, 738, DOI: 10.1109/22.989958. 
[120] B. van Grinsven, K. Eersels, M. Peeters, P. LosadaPérez, T. Vandenryt, T. J. Cleij, P. Wagner, ACS Applied Materials \& Interfaces. 2014 6, 13309, DOI: 10.1021/am503667s.

[121] T. Kodama, A. Jain, K. E. Goodson, Nano Letters. 2009 9, 2005, DOI: 10.1021/n1900272m.

[122] B. van Grinsven, N. Vanden Bon, H. Strauven, L. Grieten, M. Murib, K. L. J. Monroy, S. D. Janssens, K. Haenen, M. J. Schöning, V. Vermeeren, M. Ameloot, L. Michiels, R. Thoelen, W. de Ceuninck, P. Wagner, ACS Nano. 2012 6, 2712, DOI: 10.1021/nn300147e.

[123] B. van Grinsven, K. Eersels, O. Akkermans, S. Ellermann, A. Kordek, M. Peeters, O. Deschaume, C. Bartic, H. Diliën, E. Steen Redeker, P. Wagner, T. J. Cleij, ACS Sens. 2016 1, 1140, DOI: 10.1021/acssensors.6b00435.

[124] B. van Grinsven, K. Eersels, S. Erkens-Hulshof, H. Diliën, K. Nurekeyeva, P. Cornelis, D. Klein, F. Crijns, G. Tuijthof, P. Wagner, E. Steen Redeker, T. J. Cleij, Phys.

Status Solidi A. 2018 215, 1700777, DOI:

10.1002/pssa.201700777.

[125] K. Bers, K. Eersels, B. van Grinsven, M. Daemen, J. F. J. Bogie, J. J. A. Hendriks, E. E. Bouwmans, C. Püttmann, C. Stein, S. Barth, G. M. J. Bos, W. T. V. Germeraad, W. de Ceuninck, P. Wagner, Langmuir. 2014 30, 3631, DOI: 10.1021/la5001232.

[126] K. Eersels, B. van Grinsven, M. Khorshid, V. Somers, C. Püttmann, C. Stein, S. Barth, H. Diliën, G. M. J. Bos, W. T. V. Germeraad, T. J. Cleij, R. Thoelen, W. de Ceuninck, P. Wagner, Langmuir. 2015 31, 2043, DOI: 10.1021/la5046173. 
[127] K. Betlem, F. Canfarotta, R. Raumbault, C. E. Banks, K. Eersels, B. van Grinsven, T. J. Cleij, R. Crapnell, A. Hudson, M. Peeters, The Analyst. 2020 145, 5419, DOI: 10.1039/d0an01046d.

[128] M. Peeters, P. Csipai, B. Geerets, A. Weustenraed, B. van Grinsven, R. Thoelen, J. Gruber, W. de Ceuninck, T. J. Cleij, F. J. Troost, P. Wagner, Analytical and bioanalytical chemistry. 2013 405, 6453, DOI: 10.1007/s00216-013-70249.

[129] G. Wackers, T. Vandenryt, P. Cornelis, E. Kellens, R. Thoelen, W. de Ceuninck, P. Losada-Pérez, B. van Grinsven, M. Peeters, P. Wagner, Sensors. 2014 14, 11016, DOI:

10.3390/s140611016.

[130] H. Diliën, M. Peeters, J. Royakkers, J. Harings, P. Cornelis, P. Wagner, E. Steen Redeker, C. E. Banks, K. Eersels, B. van Grinsven, T. J. Cleij, ACS Sens. 2017 2, 583, DOI: $10.1021 /$ acssensors.7b00104.

[131] M. M. Peeters, B. van Grinsven, C. W. Foster, T. J. Cleij, C. E. Banks, Molecules (Basel, Switzerland). 2016 21, DOI: 10.3390/molecules21050552.

[132] Qin. 1999, 1.

[133] A.-G. Niculescu, C. Chircov, A. C. Bîrcă, A. M. Grumezescu, International journal of molecular sciences. 2021 22, DOI: 10.3390/ijms22042011.

[134] G. M. Whitesides, Nature. 2006 442, 368, DOI: 10.1038/nature05058. 
References

[135] B. Gale, A. Jafek, C. Lambert, B. Goenner, H.

Moghimifam, U. Nze, S. Kamarapu, Inventions. 2018 3, 60, DOI: 10.3390/inventions3030060.

[136] N. Convery, N. Gadegaard, Micro and Nano Engineering. 2019 2, 76, DOI: 10.1016/j.mne.2019.01.003.

[137] A. Manz, N. Graber, H. M. Widmer, Sensors and Actuators B: Chemical. 1990 1, 244, DOI: 10.1016/09254005(90)80209-I.

[138] Y. Song, J. Hormes, Kumar, Challa S. S. R., Small. 2008 4, 698, DOI: 10.1002/smll.200701029.

[139] H. Shi, K. Nie, B. Dong, M. Long, H. Xu, Z. Liu, Chemical engineering journal (Lausanne, Switzerland : 1996). 2019 361, 635, DOI: 10.1016/j.cej.2018.12.104.

[140] P. Sengupta, K. Khanra, A. R. Chowdhury, P. Datta, Lab-on-a-chip sensing devices for biomedical applications, Woodhead Publishing, Oxford 2019, 47.

[141] W. Sun, Z. Luo, J. Lee, H.-J. Kim, K. Lee, P. Tebon, Y. Feng, M. R. Dokmeci, S. Sengupta, A. Khademhosseini, Advanced Healthcare Materials. 2019 8, e1801363, DOI: 10.1002/adhm.201801363.

[142] E. Moradi, S. Jalili-Firoozinezhad, M. Solati-Hashjin, Acta Biomaterialia. 2020 116, 67, DOI:

10.1016/j.actbio.2020.08.041.

[143] O. Reynolds, Philosophical Transactions of the Royal Society of London. $\mathbf{1 8 8 3}$ 174, 935, DOI:

10.1098/rstl.1883.0029. 
[144] C. L. Hansen, E. Skordalakes, J. M. Berger, S. R. Quake, Proceedings of the National Academy of Sciences of the United States of America. 2002 99, 16531, DOI: 10.1073/pnas.262485199.

[145] J. Happel, H. Brenner, Low Reynolds number hydrodynamics, Martinus Nijhoff Publishers, The Hague, Boston, Lancaster 1983.

[146] R. R. G. Soares, P. Novo, A. M. Azevedo, P. Fernandes, M. R. Aires-Barros, V. Chu, J. P. Conde, Lab Chip. 2014 14, 4284, DOI: 10.1039/c4lc00695j.

[147] M. Dziubinski, in Advances in Microfluidics InTech 2012.

[148] S. Sohrabi, N. kassir, M. Keshavarz Moraveji, RSC $A d v .2020$ 10, 27560, DOI: 10.1039/D0RA04566G.

[149] S. O. Rastegar, T. Gu, Journal of chromatography. A. 2017 1490, 133, DOI: 10.1016/j.chroma.2017.02.026.

[150] S. V. Patankar, Numerical heat transfer and fluid flow, CRC Press, Boca Raton 2018?

[151] F. P. Incropera, D. P. DeWitt, Fundamentals of heat and mass transfer, Wiley, New York 1990.

[152] V. Faustino, S. O. Catarino, R. Lima, G. Minas, Journal of Biomechanics. 2016 49, 2280, DOI:

10.1016/j.jbiomech.2015.11.031.

[153] X.-M. Zhao, Y. Xia, G. M. Whitesides, Journal of Materials Chemistry. 1997 7, 1069, DOI: 10.1039/a700145b. 
References

[154] G. M. Whitesides, E. Ostuni, S. Takayama, X. Jiang, D. E. Ingber, Annual Review of Biomedical Engineering. 2001 3, 335, DOI: 10.1146/annurev.bioeng.3.1.335.

[155] D. Qin, Y. Xia, G. M. Whitesides, Nature Protocols. 2010 5, 491, DOI: 10.1038/nprot.2009.234.

[156] R.-H. Chen, C.-M. Cheng, in: Proceedings26th Annual International Symposium on Microlithography, Santa Clara, CA, Sunday 25 February 2001, in Advances in Resist Technology and Processing XVIII, SPIE Proceedings (SPIE, 2001), p. 494.

[157] D. I. Walsh, D. S. Kong, S. K. Murthy, P. A. Carr, Trends in biotechnology. 2017 35, 383, DOI:

10.1016/j.tibtech.2017.01.001.

[158] Kim, Biochip J. 2008 2, 1.

[159] M. A. Unger, H. P. Chou, T. Thorsen, A. Scherer, S.

R. Quake, Science. 2000 288, 113, DOI:

10.1126/science.288.5463.113.

[160] J. C. McDonald, D. C. Duffy, J. R. Anderson, D. T. Chiu, H. Wu, O. J. A. Schueller, G. M. Whitesides, Electrophoresis. 2000 21, 27, DOI: 10.1002/(SICI)15222683(20000101)21:1<27::AID-ELPS27>3.0.CO;2-C.

[161] K. Ren, J. Zhou, H. Wu, Accounts of Chemical Research. 2013 46, 2396, DOI: 10.1021/ar300314s.

[162] C. Rivet, H. Lee, A. Hirsch, S. Hamilton, H. Lu, Chemical Engineering Science. 2011 66, 1490, DOI: 10.1016/j.ces.2010.08.015. 
[163] J. B. Nielsen, R. L. Hanson, H. M. Almughamsi, C. Pang, T. R. Fish, A. T. Woolley, Anal. Chem. 2020 92, 150, DOI: 10.1021/acs.analchem.9b04986.

[164] W. Gu, X. Zhu, N. Futai, B. S. Cho, S. Takayama, Proceedings of the National Academy of Sciences of the United States of America. 2004 101, 15861, DOI: 10.1073/pnas.0404353101.

[165] S. W. Park, W. I. Lee, S. N. Moon, Y.-E. Yoo, Y. H. Cho, Express Polym. Lett. 2011 5, 950, DOI:

10.3144/expresspolymlett.2011.93.

[166] L. Morelli, L. Serioli, F. A. Centorbi, C. B. Jendresen, M. Matteucci, O. Ilchenko, D. Demarchi, A. T. Nielsen, K. Zór, A. Boisen, Lab on a Chip. 2018 18, 869, DOI: 10.1039/C7LC01217A.

[167] G. S. Fiorini, D. T. Chiu, BioTechniques. 2005 38, 429, DOI: 10.2144/05383RV02.

[168] U. M. Attia, S. Marson, J. R. Alcock, Microfluidics and Nanofluidics. 2009 7, 1, DOI: 10.1007/s10404-009-0421$\mathrm{x}$.

[169] J. Wu, M. Gu, Journal of Biomedical Optics. 2011 16, 80901, DOI: $10.1117 / 1.3607430$.

[170] M. Focke, D. Kosse, C. Müller, H. Reinecke, R. Zengerle, F. von Stetten, Lab Chip. 2010 10, 1365, DOI: 10.1039/c001195a.

[171] D. J. Guckenberger, T. E. de Groot, A. M. D. Wan, D. J. Beebe, E. W. K. Young, Lab on a Chip. 2015 15, 2364, DOI: 10.1039/C5LC00234F. 
References

[172] Y. Temiz, R. D. Lovchik, G. V. Kaigala, E.

Delamarche, Microelectronic Engineering. 2015 132, 156,

DOI: 10.1016/j.mee.2014.10.013.

[173] A. R. Jafek, S. Harbertson, H. Brady, R. Samuel, B.

K. Gale, Anal. Chem. 2018 90, 7190, DOI:

10.1021/acs.analchem.7b05176.

[174] D. Patko, Z. Mártonfalvi, B. Kovacs, F. Vonderviszt, M. Kellermayer, R. Horvath, Sensors and Actuators B:

Chemical. 2014 196, 352, DOI: 10.1016/j.snb.2014.01.107.

[175] M. Serra, I. Pereiro, A. Yamada, J.-L. Viovy, S.

Descroix, D. Ferraro, Lab Chip. 2017 17, 629, DOI:

10.1039/C6LC01319H.

[176] A. K. Au, W. Huynh, L. F. Horowitz, A. Folch, Angewandte Chemie International Edition. 2016 55, 3862, DOI: 10.1002/anie.201504382.

[177] N. Bhattacharjee, A. Urrios, S. Kang, A. Folch, Lab Chip. 2016 16, 1720, DOI: 10.1039/C6LC00163G.

[178] S. Waheed, J. M. Cabot, N. P. Macdonald, T. Lewis, R. M. Guijt, B. Paull, M. C. Breadmore, Lab Chip. 2016 16, 1993, DOI: 10.1039/C6LC00284F.

[179] N. Shahrubudin, T. C. Lee, R. Ramlan, Procedia Manufacturing. 2019 35, 1286, DOI:

10.1016/j.promfg.2019.06.089.

[180] Z. Liu, Y. Wang, B. Wu, C. Cui, Y. Guo, C. Yan, The International Journal of Advanced Manufacturing Technology. 2019 102, 2877, DOI: 10.1007/s00170-01903332-x. 


\subsection{References for Chapter 2: Point of Care Diagnostics in Resource-Limited Settings}

[1] A. Boutayeb, in The handbook of disease burdens and quality of life measures Springer, New York 2010, pp. 11711188.

[2] D. Kuupiel, V. Bawontuo, P. K. Drain, N. Gwala, T. $\mathrm{P}$. Mashamba-Thompson, BMC health services research. 2019 19, 519, DOI: 10.1186/s12913-019-4351-3.

[3] A. Starr, K. M. Graef, J. Dent, Pharmaceutical patent analyst. 2016 5, 391, DOI: 10.4155/ppa-2016-0038.

[4] D. Migliozzi, T. Guibentif, Biosensors. 2018 8, DOI: 10.3390/bios8040119.

[5] N. P. Pai, C. Vadnais, C. Denkinger, N. Engel, M.

Pai, PLoS medicine. 2012 9, e1001306, DOI:

10.1371/journal.pmed.1001306.

[6] S. K. Sia, L. J. Kricka, Lab on a chip. 2008 8, 1982, DOI: 10.1039/B817915H.

[7] T. R. Kozel, A. R. Burnham-Marusich, Journal of clinical microbiology. 2017 55, 2313, DOI:

10.1128/JCM.00476-17.

[8] O. Pashchenko, T. Shelby, T. Banerjee, S. Santra, ACS infectious diseases. 2018 4, 1162, DOI:

10.1021/acsinfecdis.8b00023. 
References

[9] J. W. Lowdon, K. Eersels, R. Rogosic, T. Boonen, B. Heidt, H. Diliën, B. van Grinsven, T. J. Cleij, Sensors and Actuators A: Physical. 2019 295, 586, DOI:

10.1016/j.sna.2019.06.029.

[10] T. Vandenryt, B. van Grinsven, K. Eersels, P. Cornelis, S. Kholwadia, T. J. Cleij, R. Thoelen, W. de Ceuninck, M. Peeters, P. Wagner, Sensors (Basel, Switzerland). 2017 17, DOI: 10.3390/s17122701.

[11] K. Takemura, O. Adegoke, N. Takahashi, T. Kato, T.C. Li, N. Kitamoto, T. Tanaka, T. Suzuki, E. Y. Park, Biosensors \& bioelectronics. 2017 89, 998, DOI:

10.1016/j.bios.2016.10.045.

[12] J. W. Lowdon, K. Eersels, R. Rogosic, B. Heidt, H. Diliën, E. S. Redeker, M. Peeters, B. van Grinsven, T. J. Cleij, Sensors and actuators. B, Chemical. 2019 282, 137, DOI: 10.1016/j.snb.2018.11.053.

[13] L.-H. Xiong, R. Cui, Z.-L. Zhang, X. Yu, Z. Xie, Y.B. Shi, D.-W. Pang, ACS nano. 2014 8, 5116, DOI: 10.1021/nn501174g.

[14] F. B. Myers, L. P. Lee, Lab on a chip. 2008 8, 2015, DOI: $10.1039 / \mathrm{b} 812343 \mathrm{~h}$.

[15] S. Afsahi, M. B. Lerner, J. M. Goldstein, J. Lee, X. Tang, D. A. Bagarozzi, D. Pan, L. Locascio, A. Walker, F. Barron, B. R. Goldsmith, Biosensors \& bioelectronics. 2018 100, 85, DOI: 10.1016/j.bios.2017.08.051.

[16] S. Campuzano, P. Yáñez-Sedeño, J. M. Pingarrón, Sensors (Basel, Switzerland). 2017 17, DOI: 10.3390/s17112533. 
[17] J. Cecchetto, F. C. B. Fernandes, R. Lopes, P. R. Bueno, Biosensors \& bioelectronics. 2017 87, 949, DOI: 10.1016/j.bios.2016.08.097.

[18] K. Hsieh, B. S. Ferguson, M. Eisenstein, K. W. Plaxco, H. T. Soh, Accounts of chemical research. 2015 48, 911, DOI: 10.1021/ar500456w.

[19] J. Y. Park, L. J. Kricka, Lab on a chip. 2007 7, 547, DOI: 10.1039/B702667F.

[20] D. Kuupiel, V. Bawontuo, T. P. MashambaThompson, Diagnostics (Basel, Switzerland). 2017 7, DOI: 10.3390/diagnostics7040058.

[21] S. Wang, M. A. Lifson, F. Inci, L.-G. Liang, Y.-F. Sheng, U. Demirci, Expert review of molecular diagnostics. 2016 16, 449, DOI: 10.1586/14737159.2016.1142877.

[22] M. Urdea, L. A. Penny, S. S. Olmsted, M. Y. Giovanni, P. Kaspar, A. Shepherd, P. Wilson, C. A. Dahl, S. Buchsbaum, G. Moeller, D. C. Hay Burgess, Nature. 2006 444 Suppl 1, 73, DOI: 10.1038/nature05448.

[23] A. N. Abou Tayoun, B. P. Ward, G. Maltezos, A. Scherer, G. J. Tsongalis, Experimental and molecular pathology. 2012 93, 261, DOI: 10.1016/j.yexmp.2012.05.002.

[24] Y. Furuse, Proceedings of the National Academy of Sciences of the United States of America 2019 116, 478, DOI: 10.1073/pnas.1814484116.

[25] A. M. Elliott, N. Luo, G. Tembo, B. Halwiindi, G. Steenbergen, L. Machiels, J. Pobee, P. Nunn, R. J. Hayes, K. P. McAdam, BMJ (Clinical research ed.). 1990 301, 412, DOI: 10.1136/bmj.301.6749.412. 
References

[26] R. McNerney, Diagnostics (Basel, Switzerland). 2015 5, 200, DOI: 10.3390/diagnostics5020200.

[27] R. Hecht, J. Stover, L. Bollinger, F. Muhib, K. Case, D. de Ferranti, The Lancet. 2010 376, 1254, DOI: 10.1016/S0140-6736(10)61255-X.

[28] F. W. Kimani, S. M. Mwangi, B. J. Kwasa, A. M. Kusow, B. K. Ngugi, J. Chen, X. Liu, R. Cademartiri, M. M. Thuo, Micromachines. 2017 8, DOI: 10.3390/mi8110317.

[29] Expedited Programs for Serious Conditions-Drugs and Biologics. Available online:

https://www.fda.gov/regulatory-information/search-fdaguidance-documents/expedited-programs-serious-conditionsdrugs-and-biologics (accessed on 9 July 2020).

[30] D. B. Ridley, S. A. Régnier, Health affairs (Project Hope). 2016 35, 776, DOI: 10.1377/hlthaff.2015.1314.

[31] B. T. Slingsby, K. Kurokawa, The Lancet Global Health. 2013 1, e184-e185, DOI: 10.1016/S2214109X(13)70055-X.

[32] T. Costa Barbosa Bessa, E. Santos de Aragão, J. M. Medeiros Guimarães, B. de Araújo Almeida, BioMed research international. 2017 2017, 4765719, DOI: $10.1155 / 2017 / 4765719$.

[33] M. A. Mercer, S. M. Thompson, R. M. de Araujo, International journal of health services : planning, administration, evaluation. 2014 44, 323, DOI: 10.2190/HS.44.2.i. 
[34] R. E. Black, M. K. Bhan, M. Chopra, I. Rudan, C. G. Victora, The Lancet. 2009 373, 1584, DOI: 10.1016/S01406736(09)60886-2.

[35] D. Guha-Sapir, The Lancet. 2009 374, 201, DOI: 10.1016/S0140-6736(09)61331-3.

[36] D. McCoy, G. Kembhavi, J. Patel, A. Luintel, The Lancet. 2009 373, 1645, DOI: 10.1016/S01406736(09)60571-7.

[37] B. C. Rao, Royal Society open science. 2019 6, 180421, DOI: 10.1098/rsos.180421.

[38] J. Prabhu, Philosophical transactions. Series A, Mathematical, physical, and engineering sciences. 2017 375, DOI: 10.1098/rsta.2016.0372.

[39] V.-T. Tran, P. Ravaud, BMC medicine. 2016 14, 102, DOI: $10.1186 / \mathrm{s} 12916-016-0651-1$.

[40] P. Howitt, A. Darzi, G.-Z. Yang, H. Ashrafian, R. Atun, J. Barlow, A. Blakemore, A. M. J. Bull, J. Car, L. Conteh, G. S. Cooke, N. Ford, S. A. J. Gregson, K. Kerr, D. King, M. Kulendran, R. A. Malkin, A. Majeed, S. Matlin, R. Merrifield, H. A. Penfold, S. D. Reid, P. C. Smith, M. M. Stevens, M. R. Templeton, C. Vincent, E. Wilson, The Lancet. 2012 380, 507, DOI: 10.1016/S0140-6736(12)611271.

[41] B. Heidt, R. Rogosic, S. Bonni, J. Passariello-Jansen, D. Dimech, J. W. Lowdon, R. Arreguin-Campos, E. Steen Redeker, K. Eersels, H. Diliën, B. van Grinsven, T. J. Cleij, Phys. Status Solidi A. 2020, 1900935, DOI:

10.1002/pssa.201900935. 
References

[42] Z. Kleinman, BBC News. 3/16/2020.

[43] A. N. Abou Tayoun, P. R. Burchard, I. Malik, A.

Scherer, G. J. Tsongalis, American journal of clinical

pathology. 2014 141, 17, DOI:

10.1309/AJCPA1L4KPXBJNPG.

[44] R. Zachariah, S. D. Reid, P. Chaillet, M. Massaquoi, E. J. Schouten, A. D. Harries, Tropical medicine \& international health : TM \& IH. 2011 16, 37, DOI: 10.1111/j.1365-3156.2010.02669.x.

[45] T. P. Mashamba-Thompson, N. A. Jama, B. Sartorius, P. K. Drain, R. M. Thompson, Diagnostics (Basel, Switzerland). 2017 7, DOI: 10.3390/diagnostics7010003.

[46] I. Toskin, M. Murtagh, R. W. Peeling, K. Blondeel, J. Cordero, J. Kiarie, Sexually transmitted infections. 2017 93, S69-S80, DOI: 10.1136/sextrans-2016-053071.

[47] R. W. Peeling, K. K. Holmes, D. Mabey, A. Ronald, Sexually transmitted infections. 200682 Suppl 5, v1-6, DOI: 10.1136/sti.2006.024265.

[48] R. W. Peeling, D. Mabey, A. Herring, E. W. Hook, Nature reviews. Microbiology. 2006 4, S7-19, DOI: 10.1038/nrmicro1569.

[49] R. Rasti, D. Nanjebe, J. Karlström, C. Muchunguzi, J. Mwanga-Amumpaire, J. Gantelius, A. Mårtensson, L. Rivas, F. Galban, P. Reuterswärd, H. Andersson Svahn, H. M. Alvesson, Y. Boum, T. Alfvén, PloS one. 2017 12, e0182005, DOI: 10.1371/journal.pone.0182005.

[50] L. Duchesne, G. Hejblum, N. C. Toure Kane, R. Njouom, T.-D. Toni, R. Moh, B. Sylla, N. Rouveau, A. Attia, 
K. Lacombe, The International journal on drug policy. 2020 75, 102613, DOI: 10.1016/j.drugpo.2019.102613.

[51] T. L. Gift, M. S. Pate, E. W. Hook, W. J. Kassler, Sexually transmitted diseases. 1999 26, 232, DOI: 10.1097/00007435-199904000-00010.

[52] C. M. Pandey, S. Augustine, S. Kumar, S. Kumar, S. Nara, S. Srivastava, B. D. Malhotra, Biotechnology journal. 2018 13, DOI: 10.1002/biot.201700047.

[53] M. Azhar, D. Dendukuri, in Medical biosensors for point of care (POC) applications Woodhead Publishing is an imprint of Elsevier, Duxford, United Kingdom 2016, pp. 255273.

[54] C. D. Chin, V. Linder, S. K. Sia, Lab on a chip. 2012 12, 2118, DOI: 10.1039/C2LC21204H.

[55] S. Sharma, J. Zapatero-Rodríguez, P. Estrela, R. O'Kennedy, Biosensors. 2015 5, 577, DOI: 10.3390/bios5030577.

[56] A. Zhdanov, J. Keefe, L. Franco-Waite, K. R.

Konnaiyan, A. Pyayt, Biosensors \& bioelectronics. 2018 103, 138, DOI: 10.1016/j.bios.2017.12.033.

[57] E. Archibong, K. R. Konnaiyan, H. Kaplan, A. Pyayt, Biosensors \& bioelectronics. 2017 88, 204, DOI:

10.1016/j.bios.2016.08.030.

[58] D. Quesada-González, A. Merkoçi, Biosensors \& bioelectronics. 2017 92, 549, DOI:

10.1016/j.bios.2016.10.062. 
References

[59] A. C. Sun, C. Yao, A. G. Venkatesh, D. A. Hall, Sensors and actuators. B, Chemical. 2016 235, 126, DOI: 10.1016/j.snb.2016.05.010.

[60] J. L. Delaney, C. F. Hogan, Methods in molecular biology (Clifton, N.J.). 2015 1256, 277, DOI: 10.1007/978-14939-2172-0_19.

[61] A. Sabet Sarvestani, K. H. Sienko, Globalization and health. 2018 14, 65, DOI: 10.1186/s12992-018-0355-8.

[62] N. Parvizi, K. Woods, Clinical Medicine. 2014 14, 6, DOI: 10.7861/clinmedicine.14-1-6.

[63] S. Basu, J. C. Hassenplug, The New England journal of medicine. 2012 367, 485, DOI: 10.1056/NEJMp1204170.

[64] R. Ramamoorthi, K. M. Graef, J. Dent, International journal for parasitology. Drugs and drug resistance. 2014 4, 220, DOI: 10.1016/j.ijpddr.2014.09.002.

[65] C. K. Manner, K. M. Graef, J. Dent, Tropical medicine and infectious disease. 2019 4, DOI:

10.3390/tropicalmed4010053.

[66] R. Peeling, Expert review of molecular diagnostics. 2015 15, 1107, DOI: 10.1586/14737159.2015.1081802.

[67] S. Jarosławski, M. Pai, Journal of epidemiology and global health. 2012 2, 39, DOI: 10.1016/j.jegh.2011.12.001.

[68] N. A. Baloch, M. Pai, The Lancet Infectious Diseases. 2012 12, 579, DOI: 10.1016/S1473-3099(12)70122-6.

[69] E. Marseille, B. Larson, D. S. Kazi, J. G. Kahn, S. Rosen, Bulletin of the World Health Organization. 2015 93, 118, DOI: 10.2471/BLT.14.138206. 
[70] N. G. Campos, V. Tsu, J. Jeronimo, M. Mvundura, J. J. Kim, BMC cancer. 2017 17, 791, DOI: 10.1186/s12885017-3786-3.

[71] K. Simeon, M. Sharma, J. Dorward, J. Naidoo, N. Dlamini, P. Moodley, N. Samsunder, R. V. Barnabas, N. Garrett, P. K. Drain, PloS one. 2019 14, e0223669, DOI: 10.1371/journal.pone.0223669.

[72] A. N. Phillips, V. Cambiano, F. Nakagawa, D. Ford, T. Apollo, J. Murungu, C. Rousseau, G. Garnett, P. Ehrenkranz, L. Bansi-Matharu, L. Vojnov, Z. Katz, R. Peeling, P. Revill, Open forum infectious diseases. 2016 3, ofw161, DOI: 10.1093/ofid/ofw161.

[73] E. P. Hyle, I. V. Jani, J. Lehe, A. E. Su, R. Wood, J. Quevedo, E. Losina, I. V. Bassett, P. P. Pei, A. D. Paltiel, S. Resch, K. A. Freedberg, T. Peter, R. P. Walensky, PLoS medicine. 2014 11, e1001725, DOI:

10.1371/journal.pmed.1001725.

[74] J. Estill, M. Egger, N. Blaser, L. S. Vizcaya, D.

Garone, R. Wood, J. Campbell, T. B. Hallett, O. Keiser, AIDS (London, England). 2013 27, 1483, DOI:

10.1097/QAD.0b013e328360a4e5.

[75] B. E. Nichols, S. J. Girdwood, T. Crompton, L. Stewart-Isherwood, L. Berrie, D. Chimhamhiwa, C. Moyo, J. Kuehnle, W. Stevens, S. Rosen, Journal of the International AIDS Society. 2019 22, e25337, DOI: 10.1002/jia2.25337.

[76] S. J. Girdwood, B. E. Nichols, C. Moyo, T.

Crompton, D. Chimhamhiwa, S. Rosen, PloS one. 2019 14, e0221586, DOI: 10.1371/journal.pone.0221586. 
References

[77] A. Smith, M. Sabidó, E. Camey, A. Batres, J. Casabona, International journal of gynaecology and obstetrics: the official organ of the International Federation of Gynaecology and Obstetrics. 2015130 Suppl 1, S70-2, DOI: 10.1016/j.ijgo.2015.04.009.

[78] K. Yao, W. Wafula, E. C. Bile, R. Cheignsong, S. Howard, A. Demby, J. Nkengasong, American journal of clinical pathology. 2010 134, 568, DOI: 10.1309/AJCPOPXR8MNTZ5PY.

[79] N. Engel, K. Wachter, M. Pai, J. Gallarda, C. Boehme, I. Celentano, R. Weintraub, BMJ global health. 2016 1, e000132, DOI: 10.1136/bmjgh-2016-000132.

[80] E. Ackerman, E. Strickland, IEEE Spectr. 2018 55, 34, DOI: 10.1109/MSPEC.2018.8241731.

[81] E. Ackerman, M. Koziol, IEEE Spectr. 2019 56, 24, DOI: 10.1109/MSPEC.2019.8701196.

[82] C. Rustici, MedicalExpo e-Magazine. 13-6-2020.

[83] N. Lewis, Business Insider. 5/12/2020.

[84] J. E. Scott, Proceedings of the Hawaii International Conference on System Sciences 2017 (HICSS-50), 2017.

[85] F. Kaindjee-Tjituka, S. Sawadogo, G. Mutandi, A. D. Maher, N. Salomo, C. Mbapaha, M. Neo, A. Beukes, J. Gweshe, A. Muadinohamba, D. W. Lowrance, African journal of laboratory medicine. 2017 6, 643, DOI: 10.4102/ajlm.v6i1.643.

[86] N. Hsiao, K. Stinson, L. Myer, PloS one. 2013 8, e55308, DOI: 10.1371/journal.pone.0055308. 
[87] A. L. Ciaranello, J.-E. Park, L. Ramirez-Avila, K. A. Freedberg, R. P. Walensky, V. Leroy, BMC medicine. 20119 , 59, DOI: 10.1186/1741-7015-9-59.

[88] L. Dunning, N. Hsiao, L. Myer, Journal of the International AIDS Society. 2015 18, 20235, DOI: 10.7448/IAS.18.1.20235.

[89] Z. Ndlovu, E. Fajardo, E. Mbofana, T. Maparo, D. Garone, C. Metcalf, H. Bygrave, K. Kao, S. Zinyowera, PloS one. 2018 13, e0193577, DOI: 10.1371/journal.pone.0193577.

[90] C. Zeh, C. E. Rose, S. Inzaule, M. A. Desai, F. Otieno, F. Humwa, B. Akoth, P. Omolo, R. T. Chen, Y. Kebede, T. Samandari, Journal of immunological methods. 2017 448, 44, DOI: 10.1016/j.jim.2017.05.006.

[91] N. Hsiao, L. Dunning, M. Kroon, L. Myer, PloS one. 2016 11, e0152672, DOI: 10.1371/journal.pone.0152672.

[92] M. J. Haenssgen, N. Charoenboon, T. Althaus, R. C. Greer, D. Intralawan, Y. Lubell, Social science \& medicine (1982). 2018 202, 1, DOI: 10.1016/j.socscimed.2018.02.018.

[93] P. W. Gething, F. A. Johnson, F. Frempong-Ainguah, P. Nyarko, A. Baschieri, P. Aboagye, J. Falkingham, Z.

Matthews, P. M. Atkinson, BMC public health. 2012 12, 991, DOI: 10.1186/1471-2458-12-991.

[94] K. Tayler-Smith, R. Zachariah, M. Massaquoi, M. Manzi, O. Pasulani, T. van den Akker, M. Bemelmans, A. Bauernfeind, B. Mwagomba, A. D. Harries, Transactions of the Royal Society of Tropical Medicine and Hygiene. 2010 104, 313, DOI: 10.1016/j.trstmh.2010.01.007. 
References

[95] S. D. Reid, S. J. Fidler, G. S. Cooke, Clinical epidemiology. 2013 5, 387, DOI: 10.2147/CLEP.S37069.

[96] R. Sankaranarayanan, B. M. Nene, S. S. Shastri, K. Jayant, R. Muwonge, A. M. Budukh, S. Hingmire, S. G. Malvi, R. Thorat, A. Kothari, R. Chinoy, R. Kelkar, S. Kane, S. Desai, V. R. Keskar, R. Rajeshwarkar, N. Panse, K. A. Dinshaw, The New England journal of medicine. 2009 360, 1385, DOI: 10.1056/NEJMoa0808516.

[97] S. R. Steinhubl, E. D. Muse, E. J. Topol, Science translational medicine. 2015 7, 283rv3, DOI:

10.1126/scitranslmed.aaa3487.

[98] H. Abaza, M. Marschollek, Methods of information in medicine. 2017 56, e105-e122, DOI: 10.3414/ME17-05-0003.

[99] S. K. Vashist, P. B. Luppa, L. Y. Yeo, A. Ozcan, J. H. T. Luong, Trends in biotechnology. 2015 33, 692, DOI: 10.1016/j.tibtech.2015.09.001.

[100] V. Duclos, M. Yé, K. Moubassira, H. Sanou, N. H. Sawadogo, G. Bibeau, A. Sié, Health research policy and systems. 2017 15, 47, DOI: 10.1186/s12961-017-0211-y.

[101] L. Perry, R. Malkin, Medical \& biological engineering \& computing. 2011 49, 719, DOI: 10.1007/s11517-011-0786-3.

[102] M. D. Pham, P. A. Agius, L. Romero, P. McGlynn, D. Anderson, S. M. Crowe, S. Luchters, BMC health services research. 2016 16, 343, DOI: 10.1186/s12913-016-1588-y.

[103] Global Health Worforce Alliance, WHO, The costeffectiveness of close-to-community health programmes: What do we know and where are the gaps? 
[104] WHO. Treat. Train. Retain-Task Shifting: Global Recommendations and Guidelines; WHO Document Production Services: Geneva, Switzerland, 2008; ISBN 97892-4-159631-2

[105] R. M. Scheffler, J. X. Liu, Y. Kinfu, M. R. Dal Poz, Bulletin of the World Health Organization. 2008 86, 516523B, DOI: 10.2471/blt.07.046474.

[106] L. M. Puchalski Ritchie, M. van Lettow, A. Makwakwa, A. K. Chan, J. S. Hamid, H. Kawonga, A. L. C. Martiniuk, M. J. Schull, V. van Schoor, M. Zwarenstein, J. Barnsley, S. E. Straus, Trials. 2016 17, 439, DOI: 10.1186/s13063-016-1563-2.

[107] L. M. Puchalski Ritchie, M. van Lettow, J. Barnsley, A. K. Chan, M. Joshua, A. L. C. Martiniuk, M. J. Schull, M. Zwarenstein, The international journal of tuberculosis and lung disease : the official journal of the International Union against Tuberculosis and Lung Disease. 2012 16, 1492, DOI: 10.5588/ijtld.12.0206.

[108] L. F. Schroeder, A. Elbireer, J. B. Jackson, T. K. Amukele, PloS one. 2015 10, e0134578, DOI:

10.1371/journal.pone.0134578.

[109] D. C. Mabey, K. A. Sollis, H. A. Kelly, A. S.

Benzaken, E. Bitarakwate, J. Changalucha, X.-S. Chen, Y.-P. Yin, P. J. Garcia, S. Strasser, N. Chintu, T. Pang, F. TerrisPrestholt, S. Sweeney, R. W. Peeling, PLoS medicine. 2012 9, e1001233, DOI: 10.1371/journal.pmed.1001233.

[110] E. L. Klatman, A. J. Jenkins, M. Y. Ahmedani, G. D. Ogle, The Lancet Diabetes \& Endocrinology. 2019 7, 150, DOI: $10.1016 /$ S2213-8587(18)30074-3. 
References

8.3 References for Chapter 3: Biomimetic Bacterial Identification Platform

[1] E. Steen Redeker, K. Eersels, O. Akkermans, J.

Royakkers, S. Dyson, K. Nurekeyeva, B. Ferrando, P.

Cornelis, M. Peeters, P. Wagner, H. Diliën, B. van Grinsven, T. J. Cleij, ACS Infect. Dis. 2017, 3, 388.

[2] B. Spellberg, R. Guidos, D. Gilbert, J. Bradley, H. W. Boucher, W. M. Scheld, J. G. Bartlett, J. Edwards, Jr., I. D. S. America, Clin. Infect. Dis. 2008, 46, 155.

[3] C. L. Ventola, Pharm. Ther. 2015, 40, 277.

[4] H. A. Khan, F. K. Baig, R. Mehboob, Asian Pac. J. Trop. Biomed. 2017, 7, 478.

[5] S. M. Jacobsen, D. J. Stickler, H. L. T. Mobley, M. E. Shirtliff, Clin. Microbiol. Rev. 2008, 21, 26.

[6] A. L. Flores-Mireles, J. N. Walker, M. Caparon, S. J. Hultgren, Nat. Rev. Microbiol. 2015, 13, 269.

[7] B. M. Zalewska-Piatek, Pol. J. Microbiol. 2011, 60, 279.

[8] P. Pietrucha-Dilanchian, T. M. Hooton, Microbiol. Spectr. 2016, 4.

[9] K. N. Moore, R. A. Day, M. Albers, J Clin. Nurs. 2002, 11, 568. 
[10] M. L. Wilson, L. Gaido, Clin. Infect. Dis. 2004, 38, 1150 .

[11] Y. W. Tang, N. M. Ellis, M. K. Hopkins, D. H. Smith, D. E. Dodge, D. H. Persing, J. Clin. Microbiol. 1998, 36, 3674.

[12] M. A. Croxen, R. J. Law, R. Scholz, K. M. Keeney, M. Wlodarska, B. B. Finlay, Clin. Microbiol. Rev. 2013, 26, 822.

[13] P. E. Fournier, M. Drancourt, P. Colson, J. M. Rolain, B. L. Scola, D. Raoult, Nat. Rev. Microbiol. 2013, 11, 574.

[14] F. Adzitey, N. Huda, G. R. R. Ali, 3 Biotech. 2013, 3, 97.

[15] C. R. Lowe, Biosensors 1985, 1, 3.

[16] M. Hussain, J. Wackerlig, P. A. Lieberzeit, Biosensors 2013, 3, 89.

[17] K. Haupt, K. Mosbach, Chem. Rev. 2000, 100, 2495.

[18] K. Eersels, P. A. Lieberzeit, P. Wagner, ACS Sens. 2016, 1, 1171.

[19] O. Hayden, O. F. L. Dickert, Adv. Mater. 2001, 13, 1480.

[20] K. Eersels, B. van Grinsven, A. Ethirajan, S. Timmermans, K. L. Jiménez Monroy, J. F. J. Bogie, S. Punniyakoti, T. Vandenryt, J. J. A. Hendriks, T. J. Cleij, M. J. A. Daemen, V. Somers, W. De Ceuninck, P. Wagner, ACS Appl. Mater. Interf. 2013, 5, 7258. 
References

[21] M. Peeters, B. van Grinsven, C. Foster, T. J. Cleij, C. Banks, Molecules 2016, 21, 552.

[22] B. van Grinsven, K. Eersels, O. Akkermans, S. Ellermann, A. Kordek, M. Peeters, O. Deschaume, C. Bartic, H. Diliën, E. Steen Redeker, P. Wagner, T. J. Cleij, ACS Sens. 2016, 1, 1140.

[23] B. van Grinsven, K. Eersels, S. Erkens-Hulshof, H. Diliën, K. Nurekeyeva, P. Cornelis, D. Klein, F. Crijns, G. Tuijthof, P. Wagner, E. Steen Redeker, T. J. Cleij, Phys. Stat. Sol. 2018, 215, 1700777.

[24] D. E. Nelson, K. D. Young, J. Bacteriol. 2000, 182, 1714. doi: 10.1128/JB.182.6.1714-1721.2000 Add to Citavi project by DOI

[25] K. Eersels, B. van Grinsven, T. Vandenryt, K. L. Jiménez-Monroy, M. Peeters, V. Somers, C. Püttmann, C. Stein, S. Barth, G. M. J. Bos, W. T. V. Gemeraard, H. Diliën, T. J. Cleij, R. Thoelen, W. De Ceuninck, P. Wagner, Phys. Status Solidi A 2015, 212, 1320.

[26] D. Kaye, J. Clin. Invest. 1968, 47, 2374.

[27] H. F. N. Kvitvang, T. Andreassen, T; Adam, S. G. Villas-Bôas, P. Bruheim, Anal. Chem. 2011, 83, 2705.

[28] H. Otani, M. Kaya, J. Tsujita, J. Sports Sci. Med. 2013, 12, 197.

[29] E. M. Lenz, J. Bright, I. D. Wilson, A. Hughes, J.

Morrisson, H. Lindberg, A. Lockton, J. Pharm. Biomed. Anal. 2004, 36, 841 . 
[30] H. Alfthan, C. Haglund, J. Dabek, U. H. Stenman, Clin. Chem. 1992, 38, 1981.

[31] O. Hayden, K. J. Mann, S. Krassnig, F. L. Dickert, Ang. Chem. Int. Ed. 2006, 45, 2626.

[32] K. Bers, K. Eersels, B. van Grinsven, M. Daemen, J.

F. J. Bogie, J. J. A. Hendriks, E. E. Bouwmans, C. Püttmann,

C. Stein, S. Barth, G. Bos, G. M. J. Bos, W. T. V. Gemeraad, W. De Ceuninck, P. Wagner, Langmuir 2014, 30, 3631.

[33] D. S. Ipe, E. Horton, G. C. Ulett, Front. Cell Infect. Microbiol. 2016, 6, 14.

[34] C. T. Archer, J. F. Kim, H. Jeong, J. H. Park, C. E.

Vickers, S. Y. Lee, L. K. Nielsen, BMC Genomics 2011, 12, 9.

8.4 References for Chapter 4: The Liberalization of Microfluidics

[1] J. P. Kruth, CIRP Ann. 1991, 40, 603.

[2] J. Y. Lee, J. Ann, C. K. Chua, Appl. Mater. Today 2017, 7, 120.

[3] R. A. Buswell, W. R. Leal de Silva, S. Z. Jones, J. Dirrenberger, Cem. Concr. Res. 2018, 7, 37.

[4] F. Rengier, A. Mehndiratta, H. von Tengg-Kobligk, C. M. Zechmann, R. Unterhinninghofen, H. U. Kauczor, Int. J. Comput. Assisted Radiol. Surg. 2010, 5, 335. 
References

[5] G. Comina, A. Suska, D. Filippini, Lab Chip 2014, 14, 2978.

[6] J. Li, L. Dai, L. Zhong, B. R. Ren, Y. Xu, J. Mater. Sci. 2018, 53, 1874.

[7] R. Ding, Y. Du, R. B. Goncalves, L. F. Francis, T. M. Reineke, Polym. Chem. 2019, 10, 1067.

[8] S. Badilescu, M. Packirisamy, Polymers 2012, 4, 1278.

[9] X. L. Ma, Appl. Mech. Mater. 2013, 401-403, 2116.

[10] A. A. Yazdi, A. Pomp, W. Wong, T. Nguyen, Y. Y. Pan, J. Xu, Microfluid. Nanofluid. 2016, 20, 50.

[11] A. K. Au, W. H. Huynh, L. F. Horowitz, A. Folch, Angew. Chem. Int. Ed. 2016, 55, 3862.

[12] Y. Xia, G. M. Whitesides, Annu. Rev. Mater. Sci. 1998, 28, 153.

[13] C. M. Ho, S. H. Ng, K. H. Li, J. Y. Yoon, Lab Chip $2015,15,3627$.

[14] S. V. Murphy, A. Atala, Nat. Biotechnol. 2014, 32, 773.

[15] W. Lee, D. Kwon, B. Chung, J. G. Yeol, A. Au, A. Folch, S. Jeon, Anal. Chem. 2014, 86, 6683.

[16] S. Narayan, K. Dongshin, A. A. Berlina, Lab Chip 2005, 5, 350 .

[17] C. I. Rogers, K. Qaderi, A. T. Woolley, G. P. Nordin, Biomicrofluidics 2015, 9, 016501. 
[18] A. Roda, M. Guardigli, D. Calabria, M. M.

Calabretta, L. Cevenini, E. Michelini, Analyst 2014, 139, 6494.

[19] Formalbs - Guide to Transparent 3D Printing with clear resin. https://formlabs.com/blog/3D printing-transparentparts-techniques-for-finishing-clear-resin/ (accessed: October 2019).

[20] B. H. Cumpston, S. P. Ananthavel, S. Barlow, D. L. Dyer, J. E. Ehrlich, L. L. Erskine, A. A. Heikal, S. M.

Kuebler, I. Y. Sandy Lee, D. McCord-Maughon, J. Qin, H. Röckel, M. Rumi, X. Wu, S. R. Mader, J. W. Perry, Nature 1999, 398, 51.

[21] H. Morgan, H. Morgan, D. Holmes, N. G. Green, IEEE Proc. Nanobiotechnol. 2003, 150, 76.

[22] Y. Liu, X. Jiang, Lab Chip 2017, 17, 3960.

[23] J. S. Eduardo Brás, V. Chu, M. R. Aires-Barros, J. P. Conde, P. Fernandes, Chem. Technol. Biotechnol. 2016, 92, 334.

[24] E. K. Sackmann, A. L. Fulton, D. J. Beebe, Nature 2014, 507, 181.

[25] K. Eersels, B. van Grinsven, A. Ethirajan, S. Timmermans, K. L. Jiménez Monroy, J. F. J. Bogie, S. Punniyakoti, T. Vandenryt, J. J. A. Hendriks, T. J. Cleij, M. J. Å. P. Daemen, V. Somers, W. De Ceuninck, P. Wagner, ACS Appl. Mater. Interfaces 2013, 5, 7258.

[26] K. Eersels, H. Diliën, J. W. Lowdon, E. Steen Redeker, R. Rogosic, B. Heidt, M. Peeters, P. Cornelis, P. 
Lux, C. P. Reutelingsperger, L. J. Schurgers, T. J. Cleij, B. van Grinsven, Nutrients 2018, 10, 751.

[27] R. Rogosic, J. W. Lowdon, B. Heidt, H. Diliën, K. Eersels, B. Van Grinsven, T. J. Cleij, Phys. Status Solidi A 2019, 216, 1800941.

[28] B. Heidt, R. Rogosic, J. W. Lowdon, M. DesmondKennedy, K. Jurgaityte, J. Ferrer Orri, Y. Kronshorst, S. Mendez, E. Polyakova, H. T. Rice, F. Crijns, H. Diliën, E. Steen Redeker, K. Eersels, B. van Grinsven, T. J. Cleij, Phys. Status Solidi A 2019, 216, 1800688.

\subsection{References for Chapter 5: 3D printed Microfluidics: Applications}

[1] C. E. Owens, A. J. Hart, Lab on a chip. 2018 18, 890, DOI: $10.1039 / \mathrm{C} 7 \mathrm{LC} 00951 \mathrm{H}$.

[2] R. Rogosic, B. Heidt, J. Passariello-Jansen, S. Björnör, S. Bonni, D. Dimech, R. Arreguin-Campos, J. Lowdon, K. L. Jiménez Monroy, M. Caldara, K. Eersels, B. van Grinsven, T. J. Cleij, H. Diliën, Journal of chemical education. 2021 98, 439, DOI: 10.1021/acs.jchemed.0c01115.

[3] Statista, Netherlands: number of elementary schools 2020 | Statista, https://www.statista.com/statistics/784246/number-ofelementary-schools-in-the-netherlands/, accessed: July, 2021.

[4] Statista, Netherlands: number of secondary education schools 2020 | Statista, 
https://www.statista.com/statistics/784257/number-ofsecondary-education-schools-in-the-netherlands/, accessed: July, 2021.

[5] Cbs, Trends in the Netherlands 2017 - Education, https://longreads.cbs.nl/trends17eng/society/figures/education/, accessed: July, 2021.

[6] Mad Science Promotion, https://nederland.madscience.org/en-US/promotie.aspx, accessed: July, 2021.

[7] Research and Markets, Toys and Games Retail in the Netherlands - Market Summary, Competitive Analysis and Forecast to 2025,

https://www.researchandmarkets.com/reports/5328360/toysand-games-retail-in-the-netherlands-market, accessed: July, 2021.

\subsection{References for Chapter 6: Topographical Vacuum Sealing}

[1] N. Convery, N. Gadegaard, Micro and Nano Engineering. 2019 2, 76, DOI: 10.1016/j.mne.2019.01.003.

[2] V. Faustino, S. O. Catarino, R. Lima, G. Minas, Journal of biomechanics. 2016 49, 2280, DOI:

10.1016/j.jbiomech.2015.11.031.

[3] B. Gale, A. Jafek, C. Lambert, B. Goenner, H.

Moghimifam, U. Nze, S. Kamarapu, Inventions. 2018 3, 60, DOI: 10.3390/INVENTIONS3030060. 
References

[4] D. I. Walsh, D. S. Kong, S. K. Murthy, P. A. Carr, Trends in biotechnology. 2017 35, 383, DOI:

10.1016/j.tibtech.2017.01.001.

[5] D. C. Duffy, J. C. McDonald, O. J. Schueller, G. M. Whitesides, Analytical Chemistry. 1998 70, 4974, DOI: 10.1021/ac980656z.

[6] H.-T. Nguyen, H. Thach, E. Roy, K. Huynh, C. M.-T. Perrault, Micromachines. 2018 9, DOI: 10.3390/mi9090461.

[7] C. L. do Lago, H. D. T. da Silva, C. A. Neves, J. G. A. Brito-Neto, J. A. F. da Silva, Analytical Chemistry. 2003 75, 3853, DOI: 10.1021/ac034437b.

[8] A. Liu, F. He, K. Wang, T. Zhou, Y. Lu, X. Xia, Lab Chip. 2005 5, 974, DOI: 10.1039/b502764k.

[9] M. S. Thomas, B. Millare, J. M. Clift, D. Bao, C. Hong, V. I. Vullev, Annals of biomedical engineering. 2010 38, 21, DOI: 10.1007/s10439-009-9831-x.

[10] A. Grimes, D. N. Breslauer, M. Long, J. Pegan, L. P. Lee, M. Khine, Lab Chip. 2008 8, 170, DOI: 10.1039/B711622E.

[11] K. Sollier, C. A. Mandon, K. A. Heyries, L. J. Blum, C. A. Marquette, Lab on a chip. 2009 9, 3489, DOI: $10.1039 / \mathrm{b} 913253 \mathrm{~h}$.

[12] M. Focke, D. Kosse, C. Müller, H. Reinecke, R. Zengerle, F. von Stetten, Lab on a chip. 2010 10, 1365, DOI: 10.1039/c001195a. 
[13] D. A. Bartholomeusz, R. W. Boutte, J. D. Andrade, J. Microelectromech. Syst. 2005 14, 1364, DOI:

10.1109/JMEMS.2005.859087.

[14] B. H. Weigl, R. Bardell, T. Schulte, F. Battrell, J. Hayenga, Biomedical Microdevices. 2001 3, 267, DOI: 10.1023/A:1012448412811.

[15] L. Peng, H. Wu, Y. Shu, P. Yi, Y. Deng, X. Lai, The Review of scientific instruments. 2016 87, 105120, DOI: 10.1063/1.4963907.

[16] T. Velten, H. Schuck, W. Haberer, F. Bauerfeld, Int J Adv Manuf Technol. 2010 47, 73, DOI: 10.1007/s00170-0091975-1.

[17] K. M. Weerakoon-Ratnayake, C. E. O'Neil, F. I. Uba, S. A. Soper, Lab on a chip. 2017 17, 362, DOI:

10.1039/c6lc01173j.

[18] A. Disch, C. Mueller, H. Reinecke, Annual International Conference of the IEEE Engineering in Medicine and Biology Society. IEEE Engineering in Medicine and Biology Society. Annual International Conference. 2007 2007, 6323, DOI: 10.1109/IEMBS.2007.4353801.

[19] M. Focke, F. Stumpf, B. Faltin, P. Reith, D. Bamarni, S. Wadle, C. Müller, H. Reinecke, J. Schrenzel, P. Francois, D. Mark, G. Roth, R. Zengerle, F. von Stetten, Lab on a chip . 2010 10, 2519, DOI: 10.1039/c004954a.

[20] R. Truckenmueller, S. Giselbrecht, J. L. Throne, Thermoforming Quarterly. 200625.

[21] R. Truckenmüller, S. Giselbrecht, N. Rivron, E. Gottwald, V. Saile, A. van den Berg, M. Wessling, C. van 
References

Blitterswijk, Advanced materials (Deerfield Beach, Fla.). 2011 23, 1311, DOI: 10.1002/adma.201003538.

[22] S. Waheed, J. M. Cabot, N. P. Macdonald, T. Lewis, R. M. Guijt, B. Paull, M. C. Breadmore, Lab Chip. 2016 16, 1993, DOI: 10.1039/c6lc00284f.

[23] G. Palmara, F. Frascella, I. Roppolo, A. Chiappone, A. Chiadò, Biosensors \& bioelectronics. 2020, 112849, DOI: 10.1016/j.bios.2020.112849.

[24] V. Saggiomo, A. H. Velders, Advanced science (Weinheim, Baden-Wurttemberg, Germany). 2015 2, 1500125, DOI: 10.1002/advs.201500125.

[25] B. Heidt, R. Rogosic, S. Bonni, J. Passariello-Jansen, D. Dimech, J. W. Lowdon, R. Arreguin-Campos, E. Steen Redeker, K. Eersels, H. Diliën, B. van Grinsven, T. J. Cleij, Phys. Status Solidi A. 2020 217, 1900935, DOI: 10.1002/pssa.201900935.

[26] R. Arreguin-Campos, K. Eersels, J. W. Lowdon, R. Rogosic, B. Heidt, M. Caldara, K. L. Jiménez-Monroy, H. Diliën, T. J. Cleij, B. van Grinsven, Microchemical Journal. 2021 169, 106554, DOI: 10.1016/j.microc.2021.106554.

[27] B. Heidt, R. Rogosic, J. W. Lowdon, M. DesmondKennedy, K. Jurgaityte, J. Ferrer Orri, Y. Kronshorst, S. Mendez, E. Polyakova, H. T. Rice, F. Crijns, H. Diliën, E. Steen Redeker, K. Eersels, B. van Grinsven, T. J. Cleij, Phys. Status Solidi A. 2019 216, 1800688, DOI: 10.1002/pssa.201800688.

[28] J. W. Lowdon, K. Eersels, R. Rogosic, T. Boonen, B. Heidt, H. Diliën, B. van Grinsven, T. J. Cleij, Sensors and 
Actuators A: Physical. 2019 295, 586, DOI:

10.1016/j.sna.2019.06.029.

[29] J. W. Lowdon, K. Eersels, R. Rogosic, B. Heidt, H.

Diliën, E. S. Redeker, M. Peeters, B. van Grinsven, T. J. Cleij,

Sensors and Actuators B: Chemical. 2019 282, 137, DOI:

10.1016/j.snb.2018.11.053.

[30] J. W. Lowdon, K. Eersels, R. Arreguin-Campos, M.

Caldara, B. Heidt, R. Rogosic, K. L. Jimenez-Monroy, T. J.

Cleij, H. Diliën, B. van Grinsven, Molecules (Basel,

Switzerland). 2020 25, DOI: 10.3390/molecules25225222.

[31] R. Rogosic, B. Heidt, J. Passariello-Jansen, S.

Björnör, S. Bonni, D. Dimech, R. Arreguin-Campos, J.

Lowdon, K. L. Jiménez Monroy, M. Caldara, K. Eersels, B. van Grinsven, T. J. Cleij, H. Diliën, Journal of chemical education. 2021 98, 439, DOI: 10.1021/acs.jchemed.0c01115.

[32] M. Focke, D. Kosse, D. Al-Bamerni, S. Lutz, C. Müller, H. Reinecke, R. Zengerle, F. von Stetten, J.

Micromech. Microeng. 2011 21, 115002, DOI: 10.1088/09601317/21/11/115002.

[33] B. Heidt, W. F. Siqueira, K. Eersels, H. Diliën, B. van

Grinsven, R. T. Fujiwara, T. J. Cleij, Biosensors. 2020 10, DOI: 10.3390/bios10100133.

[34] Y. Zhang, Int J Bioprint. 2019 5, 192, DOI:

10.18063/ijb.v5i2.192.

[35] J. M. Lee, M. Zhang, W. Y. Yeong, Microfluid Nanofluid. 2016 20, DOI: 10.1007/s10404-015-1688-8.

[36] X. Ruan, Y. Wang, E. Y. Kwon, L. Wang, N. Cheng, X. Niu, S. Ding, B. J. van Wie, Y. Lin, D. Du, Biosensors \& 
References

bioelectronics. 2021 184, 113238, DOI:

10.1016/j.bios.2021.113238.

[37] S. Sathish, K. Toda-Peters, A. Q. Shen, View. 2020 1, DOI: $10.1002 /$ viw2.1.

[38] M. Elbadawi, J. J. Ong, T. D. Pollard, S. Gaisford, A.

W. Basit, Adv. Funct. Mater. 2021 31, 2006407, DOI:

10.1002/adfm.202006407.

[39] F. Feng, J. He, J. Li, M. Mao, D. Li, Int J Bioprint. 2019 5, 202, DOI: 10.18063/ijb.v5i2.202.

[40] D. Ortiz-Acosta, T. Moore, in Functional Materials IntechOpen 2019. London, UK, 2019; ISBN 978-1-78984057-5 


\section{Supporting Information}

\subsection{Supporting Information : Chapter 3}

9.1.1 Study of the surface coverage of bacteria on the template stamp

The main goal of the article is to establish that the proposed thermal sensor platform is able to quantify the amount of bacteria present in urinary samples and is able to do so independent of variables such as gender, hormone balance... In order to do so, all other variables that might change the outcome of a measurement need to be addressed. Therefore, the amount of template bacteria per square centimeter on the stamp was examined in this experiment. As shown in the Figure S1, three different areas were examined by optical microscopy. The images were analyzed using Image J. To automate recognition and eliminate bias a threshold and size exclusion $(<10$ pixels, $>800$ pixels to exclude dirt) were applied. Circularity was set between $0-1$. This was done for four stamps and the results, summarized in the lower right panel of Figure S1 indicate that differences in coverage are present but too tiny to be picked up by the sensor. 


\section{Supporting Information}

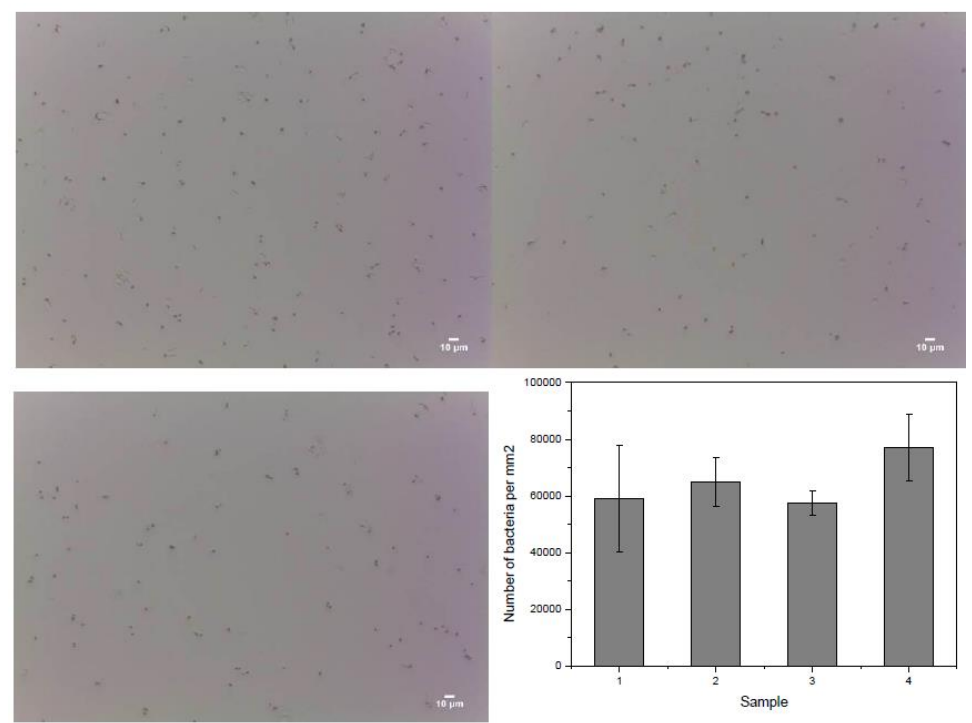

Figure S1: Coverage analysis on PDMS stamps the upper panels and lower left panel are three different areas on the stamp made to make sample 1. Three other samples were also examined and the results were summarized in the bottom right panel. 


\subsection{Supporting Information: Chapter 4}
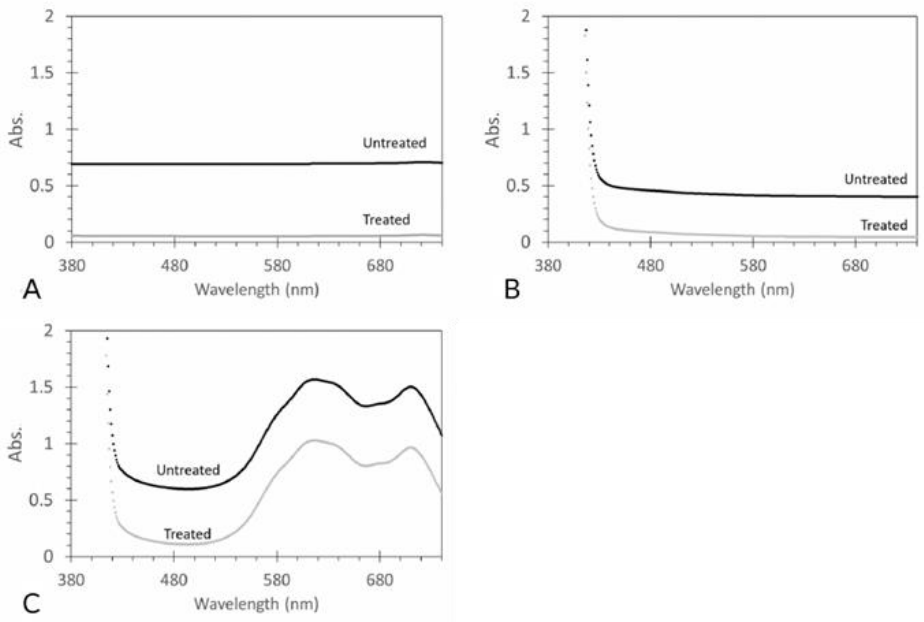

Figure $S 1$ The visible light spectrum of Clear (A) High Temperature (B) and Tough $(C)$ resin from 380-740 nm before and after the treatment process. The decrease in signal is due to decreased light scattering at the sample surface. The Tough resin shows a higher absorbance even after the treatment process, this is due to light scattering having only a minor role with the deep blue colored Tough resin, compared to the absorption at its two maxima at 616 and $710 \mathrm{~nm}$. 


\section{Supporting Information}
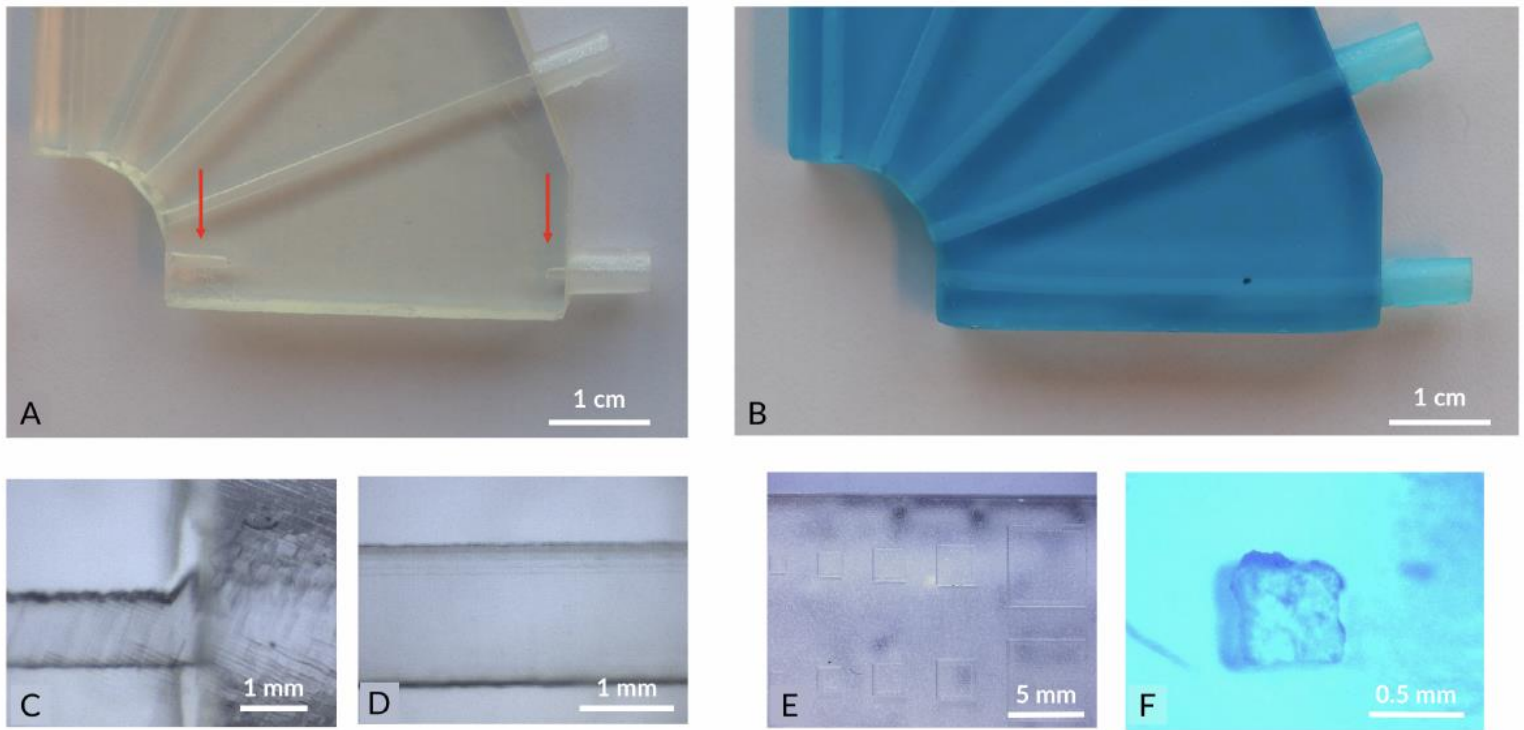

Figure $S 2$ Channel clogging seemed to start from the inside, as it was observed that only partially clogged channels where always free at both ends (A). Warping was evident at the surface horizontal to the build platform and most pronounced with the Tough resin (B). The beginning and end of channels showed a higher chance of misprinting $(C)$, as did smaller features $(E$ and $F)$. The middle part of the channels, were the measurements were conducted was usually well developed $(D)$. 

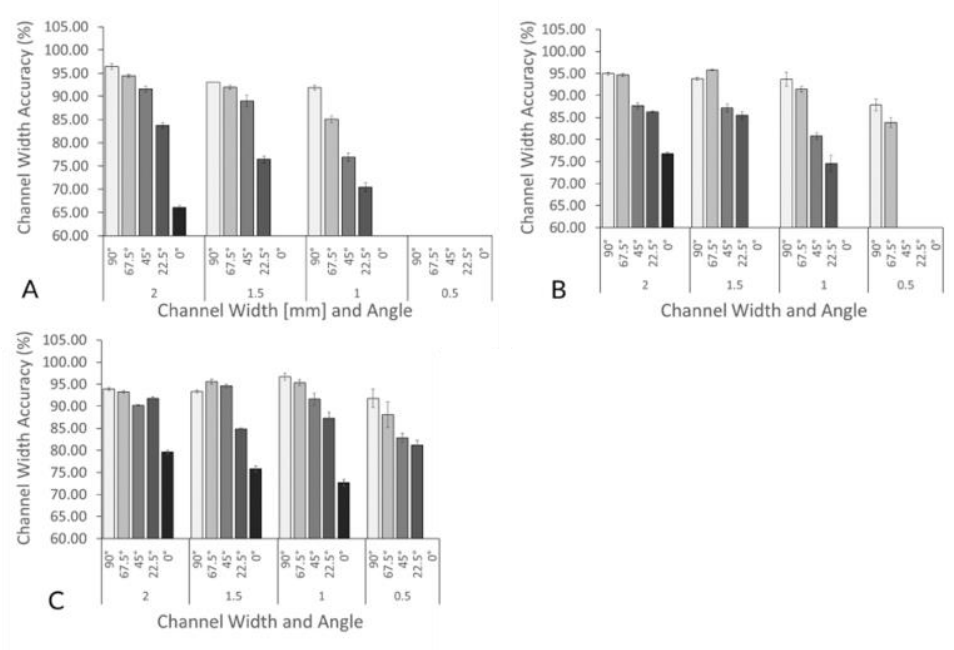

Figure $S 3$ The enclosed channel accuracy of the different resin types Clear (A), High Temperature (B) and Tough $(C)$. This $2 D$ graph contains the same information as the graph $3 D$ graph in the manuscript.
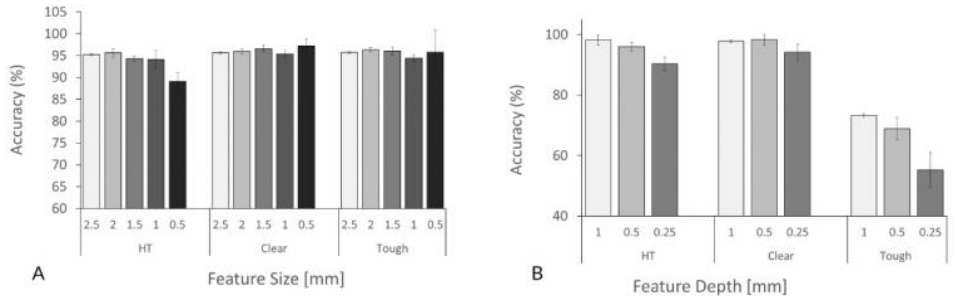

Figure $S 4$ Feature accuracy in size and depth of the different resin types. This $2 D$ graph contains the same information as the graph $3 D$ graph in the manuscript. 
Supporting Information
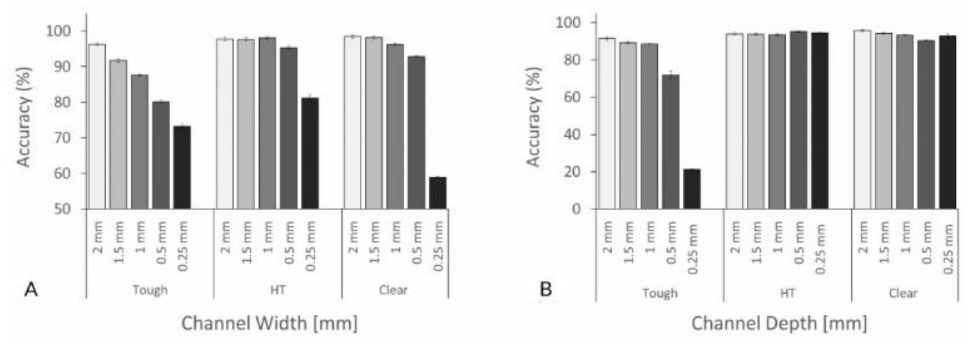

Figure S 5 Open channel accuracy for all three resins. This $2 D$ graph contains the same information as the $3 D$ graph in the manuscript.

\subsection{Supporting Information: Chapter 6}

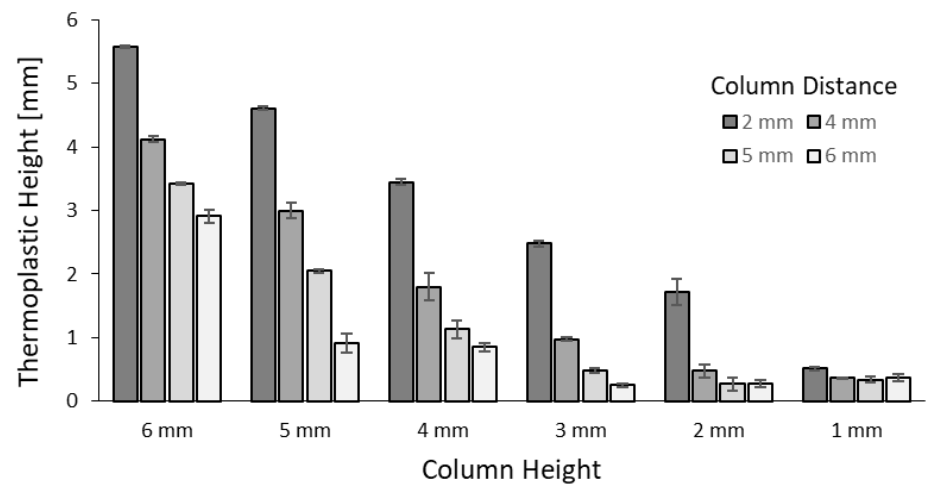

Figure S 1-Detailed graph of the macro-structure alignment shown in Figure 5. 


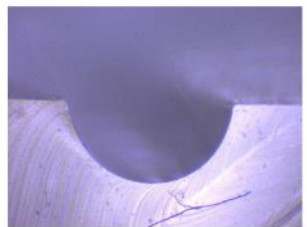

$2 \mathrm{~mm}$ Channel Size

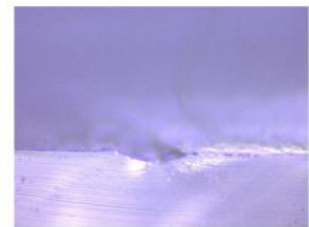

$0.8 \mathrm{~mm}$ Channel Size

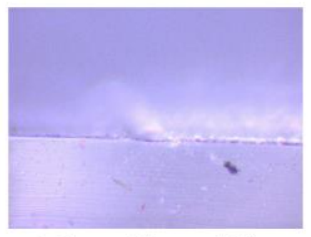

$0.5 \mathrm{~mm}$ Channel Size

Figure S 2 - Microscope images of the PDMS negatives to measure channel intrusion.
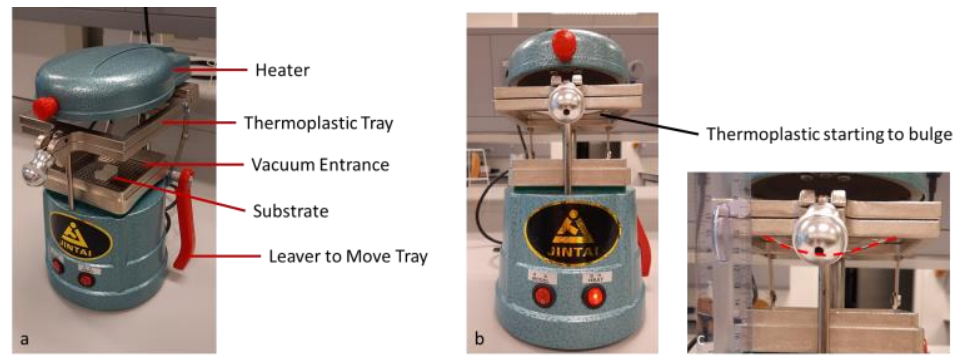

Figure S 3-The used low-cost vacuum former (a). The thermoplastic tray is moved under the heater and heated until the thermoplastic deforms and its bulge reaches $1 \mathrm{~cm}$ under the tray $(b, c)$. The tray is then lowered over the substrate using the lever and the vacuum pump activated.

Adhesive Layer

Adhesive layer was applied to a glass slide in order to show and measure its distribution and profile. Sup. figure 4 shows a microscopic image of the adhesive layer (without added thermoplastic) and sup. figure 5 shows its profile. The surface was measured with a DektakXT (Bruker) equipped with a stylus of $2 \mu \mathrm{m}$ radius and a force of $3 \mathrm{mg}$. The layer showed an average height of $1.75 \mu \mathrm{m}$ with a maximum of $7 \mu \mathrm{m}$. 
Supporting Information

Measurement directly on the 3D printed surface was difficult to conduct as the print showed a similar roughness $(2.22 \mu \mathrm{m})$ to the adhesive layer and no clear step was visible.

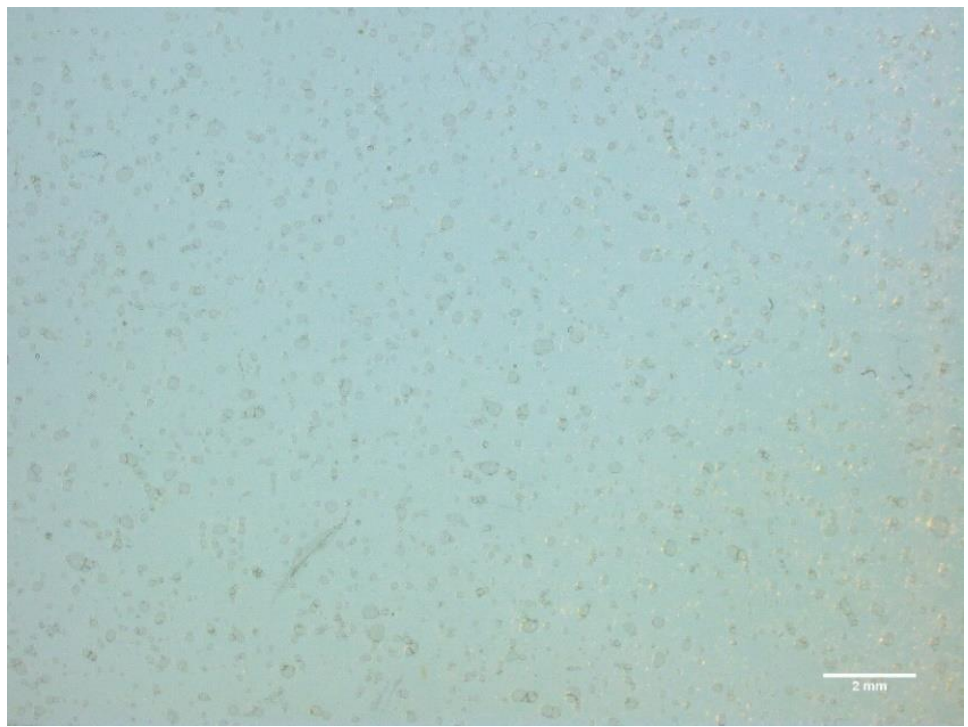

Figure S 4 - Distribution of spray adhesive after application on a glass slide and without the application of the thermoplastic. 


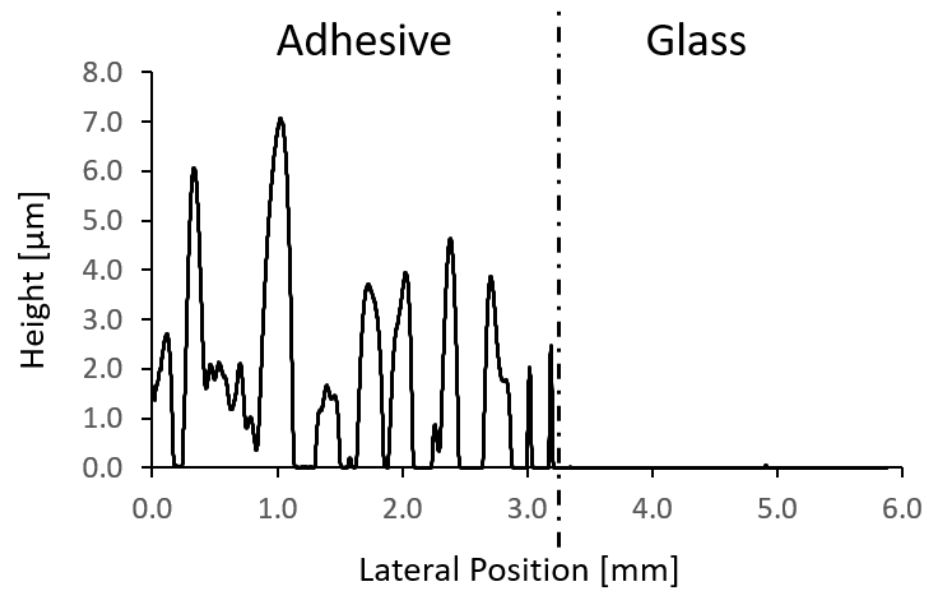

Figure S 5-Profile of the adhesive layer on a glass slide

Thermoplastic Characteristics

The dynamic mechanical analysis was performed with a DMA1 (Mettler Toledo) with a heating rate of $3{ }^{\circ} \mathrm{C}$ per minute, Displacement of $10 \mu \mathrm{m} / \mathrm{s}$, and a frequency of $1 \mathrm{~Hz}$. Sup.Figure 6 shows the complex modulus and $\tan \delta$. Sup.Figure 7 displays the storage and loss modulus.

Tan $\delta$ displays two maxima, the first one being the glass transition temperature at $75^{\circ} \mathrm{C}$ and the second one the sagging temperature at $140{ }^{\circ} \mathrm{C}$. 


\section{Supporting Information}

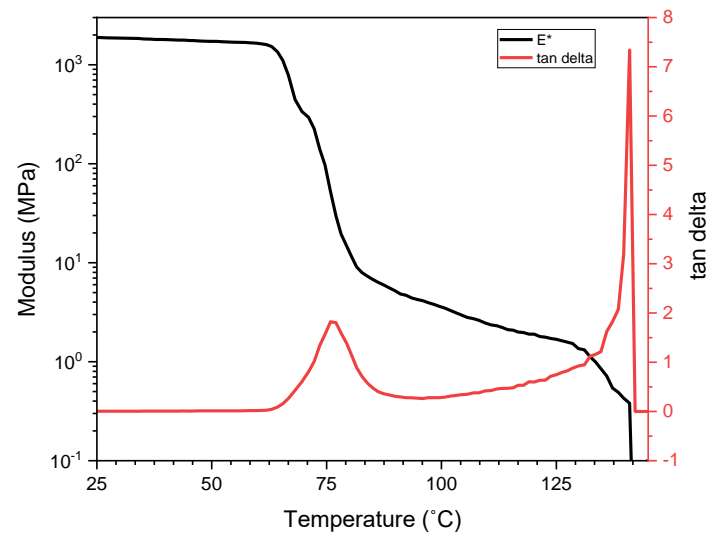

Figure $S 6$ - Complex modulus and tan $\delta$ as a function of temperature.

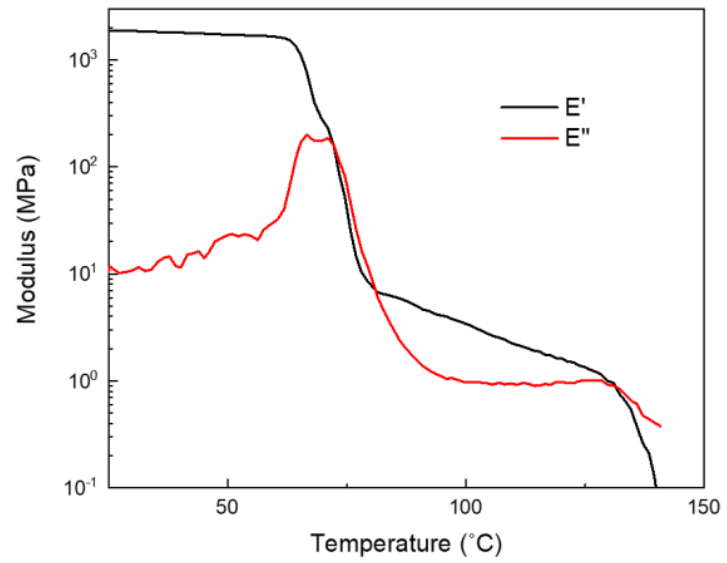

Figure S 7 - Storage Modulus E' and Loss Modulus E', as a function of temperature. 


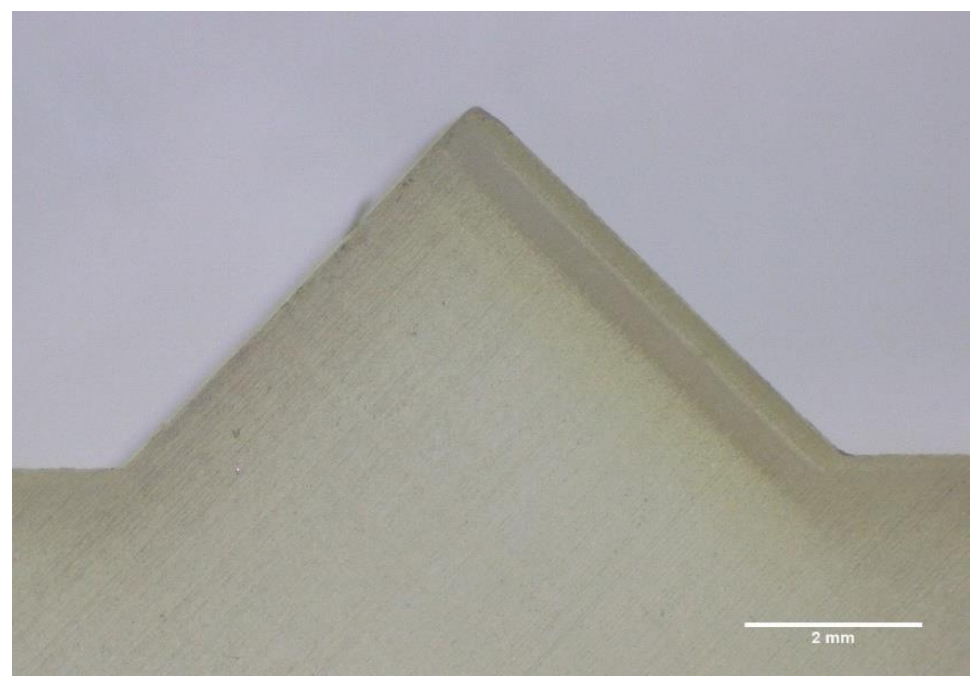

Figure S 8 - Image depicting the angle sharpness (angles: $135^{\circ}$ and $270^{\circ}$ ) of a $3 D$ print before sealing.

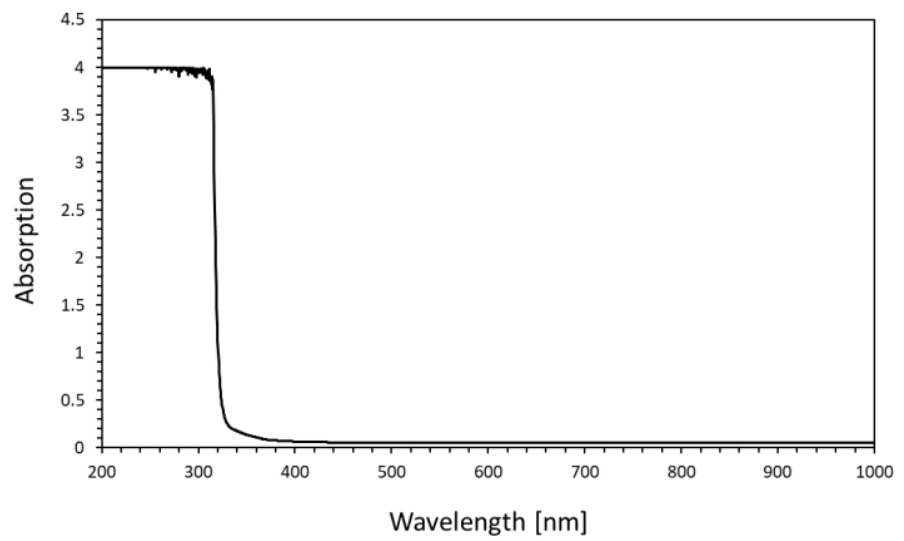

Figure S 9-Absorbance spectrum of the Polyethylene Terephthalate Glycol sheet, acquired with a Shimadzu $1900 i$ and a resolution of $0.5 \mathrm{~nm}$. 


\section{Appendices}

\subsection{Impact Paragraph}

The thesis concerns itself with questions around Point-of-Care (PoC) diagnostics, especially PoC for low-income countries (LIC). Point-of-care diagnostics are methods of disease diagnostic that can be performed fast and easy at the patientside. Here, no sample has to be sent to a laboratory for analysis, as is the case in regular diagnostics, instead, a compact, fast and self-reliant PoC device is used. The results are available within minutes, instead of the hours or days of conventional laboratory diagnostics. Examples of PoC devices that are widely used are pregnancy tests and blood-glucose meters. In high-income countries, PoC devices are researched in order to streamline the diagnostic process and save large amounts of time and money. In low-income countries on the other hand, PoC-devices could even mean the difference between life and death, since LIC often suffer from a large burden of infectious disease accompanied by an often underequipped healthcare infrastructure. Here, PoC devices are a promising tool to 'fill the gaps' in patient care, since they do not have any reliance on existing infrastructure, are cheap to manufacture, and can be distributed easily.

This thesis aims to improve the application of PoC in LIC. To this end it starts with a review article with meta-study elements that focuses on the current usage of PoC devices in LIC. The main question answered was why the many innovations that are created in the laboratory do not result in implementation into healthcare systems. While laboratories develop amazing PoC applications, the majority of devices that are actually used in 
the market are typically still based on the crude lateral flow principle used in pregnancy tests. By analyzing the problem on a social and economic level, the important factors for success or failure of PoC devices were discovered. This resulted in an open-access publication in the Journal MDPI Biosensors, which was featured as a cover-page article and later won the journals Editors-Choice award. The main findings included factors strongly intertwined with social, economic and political considerations. Problems for the application of PoC in LIC start with research, which is often performed from a high-income country perspective and not adapted to the later usage scenario in LIC. Intellectual property hurdles pose another barrier. Problematic stock management as well as low trust in the devices are further problems hindering market penetration. One of the biggest shortcomings however are the absence of valorization pathways that take every stakeholder into account. This results in unused or misused PoC devices when the intended use-case of a device is not the use-case that generates most profit for all stakeholders along the value chain.

With the new insights brought to light by this publication, researchers can make early adjustments in their design and scale-up consideration in order to improve the possibility of actual implementation of their research in LIC healthcare systems. It is crucial to make design considerations with the found themes in mind. The generated knowledge increases the likelihood of new research actually entering the market, which is the place it needs to be to make a difference in the life of patients, therefore this study can have immense impact on societies around the globe. The findings are not only interesting for researcher that develop diagnostic devices in the laboratory. Since the main focus is the use in society, the results are also applicable for social scientists, decision makers and legislators, 
as several of the problems are of structural nature that can be fought on the legislative level.

Following this, the next chapter of the thesis covers research conducted on the heat-transfer method, which lends itself for use in LIC due to its affordable setup. The work was published open-access in Physica Status Solidi A. Here a proof of application for the diagnosis of urinary tract infections was created, using several samples from different adults; E.coli were detected in urine in clinical significant ranges. It was shown, that the method is applicable regardless of gender, different levels of hydration and other metabolic factors. Additionally the importance of sample-analysis close to sample taking was demonstrated and shown how sample quality strongly declines over time. This additionally highlights the points made in the previous chapter. With this study, the potential of the heat-transfer method for actual use with different patients was shown, which is a step further towards its market implementation, for which studies of bigger scale will be the next steps.

Another important aspect of PoC devices is sample handling. The sample has to be processed, purified and transported to the sensor, for this microfluidic circuits are commonly employed. However, the creation of microfluidic systems is difficult and expensive, as it usually requires cleanroom facilities costing thousands of Euro per square meter. This creates a barrier for many research groups, especially in LIC where access to such infrastructure is more scarce. A potential solution for this is $3 \mathrm{D}$ printing. Therefore, another open-access article was published in Physica Status Solidi A where the applicability of 3D printing for the creation of microfluidic channels was benchmarked. Here a Form2 benchtop 3D printer and three 
different resin types as raw materials where used for the creation of microfluidic channels in different sizes and orientations towards the buildplate. Standard equipment and consumables were used in order to optimize the printing procedure in the most readily available setup, to promote fast adaption in other institutions and therefore create the greatest impact. It was found that size as well as the orientation of the channels have tremendous impact on channel creation. This gave valuable insights on enhancing internal channel manufacturing via resin choice and print orientation optimization. It was furthermore shown that open surfacechannels are easier to produce and can be manufactured in smaller channel sizes compared to internal channels inside the print.

Therefore, the production of open channels on the surface of a 3D print is preferred in order to produce smaller and more reliable channel. However, open channels need to be closed, which is difficult if the 3-dimensonal structure is very complex. To solve this problem, topographical vacuum sealing (Tovas), a method to easily close open surface channels on complex 3dimensional topographies, was developed. In tovas, a 3D printed substrate that features open surface channel is used together with vacuum forming. A thermoplastic sheet is heated and stretched over the 3-dimensional substrate, and vacuum applied. The pressure difference aligns the thermoplastic with the geometry of the substrate and closes the open surfacechannels. This new method enables the use of 3D printing for the creation of small surface-channels in 3D geometry. The implementation cost of this new method is very low, entry-level resin 3D printer are available for 300 Euro and a vacuum forming machine for under 100 Euro, which makes this technology especially promising for LIC. Tovas is furthermore 
Appendices

interesting for LIC, as the 3-dimensional microfluidic circuits can be easily adapted to other already existing hardware. An example given in the thesis is the manufacturing of a microfluidic cuvette that fits in a standard spectrophotometer. The manuscript for this new technology was published open access at MDPI Biosensors and was furthermore presented at the European Biosensor Symposium 2021. A patent of tovas is pending at the European Patent Office.

The work on 3D printed microfluidics presented in the thesis has the potential of an enormous impact, especially in LIC. Its low cost of implementation opens up a branch of science to a large number of research institutes that do not have access to hugely expensive cleanroom facilities. Furthermore the possibility to adapt microfluidic devices to already existing technology, will enable researcher to extend the potential of their old devices, enabling them to improve their research without large additional investments.

The gained knowledge was furthermore used to create a microfluidic measuring setup for the heat-transfer method, which was presented at the $12^{\text {th }}$ International Workshop on Engineering of Functional Interfaces. The setup was created to replace the old measurement cell, which had to be assembled by four screws and then arrested in a clutch in order to immobilize it for the experiment. To improve this process the new 3D printed system was inspired by LEGO building blocks, and is able to fit together without any screws, using LEGO-like connectors, which makes the assembly process much easier. Several addition modules were created to fit easily onto the flow-cell, like an inclined ramp and a bubble trap to prevent air from disturbing the measurement. 
Based on the success of the LEGO-inspired measuring cell, a complete system of 3D printed building blocks that feature microfluidic channels was created. These LEGO-like blocks were used to create a science education kit called Flui.Go. With our kit, students can assemble the building blocks in different ways, creating networks of microfluidic channels, mixers and reaction vessels, and then flush them with different liquids to experience scientific phenomena.

The building blocks are ideal to teach science in a natural and exciting way, as children intuitively use the blocks to recreate the experiments distributed with the kit. The modular aspect enables children to use their creativity to modify the experiments and even create completely new ones, simply by replacing or adding different blocks. Flui.Go won the competition 'Science + Engineering for Society' at Maastricht University, after securing additional funding the spin-off company Flui.Go Science was created. Since then we improved our building blocks and created a complete science kit including several different kinds of blocks and pumps. The results were published in the Journal of Chemical Education and we are currently working on another publication about the application of the microfluidic building blocks for education in primary schools. The current aim is to scale-up the production and deliver the first kits which were ordered by primary schools in the Netherlands. Given the reliance on technology in our world, STEM education is essential for a prosperous society. Therefore tools that inspire the next generation to take an interest in the STEM fields have enormous impact for the future. Our kit showed already great success in tests with primary school students and the order of 52 first kits by 26 primary schools already indicate the great impact Flui.Go will have in inspiring the next generation of scientists. 
Appendices

\subsection{Curriculum Vitae}

Benjamin Heidt was born at the 26th of April 1985 in Kehl, Germany. His first career was in economics where he received a vocational education in Management. Thereafter he decided to switch careers to pursue his passion for science. He obtained a bachelor's degree in Biochemistry at the University of Applied Sciences Mannheim in 2016, followed by a master's degree in Applied Life Sciences at the University of Applied Sciences Kaiserslautern in 2017. Over the course of his studies, he took part in a range of research projects focused on biosensing with/of/around microorganisms. In January 2018 he began to obtain his Ph.D. at the Sensor Engineering Department of the Faculty of Science and Engineering at Maastricht University. Here, his research focused on the usage and fabrication of Point-of-Care biosensors systems, which resulted in this doctoral thesis. 